\begin{tabular}{|l|l|} 
Page 1 of 2 & $\begin{array}{c}\text { 1. ECN } 633324 \\
\text { Proj. } \\
\text { ECN }\end{array}$ \\
\hline
\end{tabular}

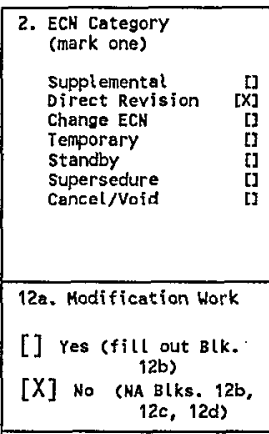

13a. Description of Change

3. Originator's Name, Organization, MsIN, and Telephone No.

Franciska H. Steen, Production

Planning and Control, T6-06, $372-2495$

6. Project Title/No./Hork Order No. Tank 241-U-106

9. Document Humbers Changed by this ECM (includes sheet no. and rev.) WHC-SD-WM-DP-191, Rev. 0

12b. Hork Package 12c. Modification Work Complete Ho. $N / A$

\section{USo Required?}

[] Yes [X] No

5. Date

$09 / 25 / 96$

7. Bldg./Sys./Fac. No.

8. Approval Designator 24I-U-106

10. Related ECN NO(s).

13. Related PO No. $N / A$

12d. Restored to Original Condi$t i o n$ (Jemp. or Standby ECN only) $N / A$

This ECN is being generated in order to update/add additional information to the existing document.

Design Authority/Cog. Engineer Signature \& Date

Design Authority/Cog. Enginee signature \& Date

13b. Design Baseline Document? [] Yes [X] No

14a. Justification (mark one)

Criteria Change $[X]$ Design Improvement

As-Found

[] Facilitate const

[] Environmental

Const. Error/Omission

14b. Justification Details

This ECN will assist in the completion of the documentation process for this document.

15. Distribution (include name, MSIN, and no. of copies)

See attached distribution.

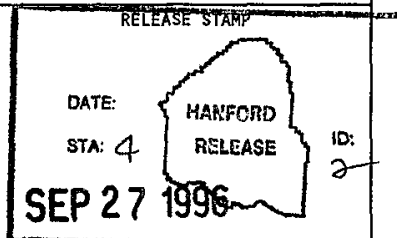




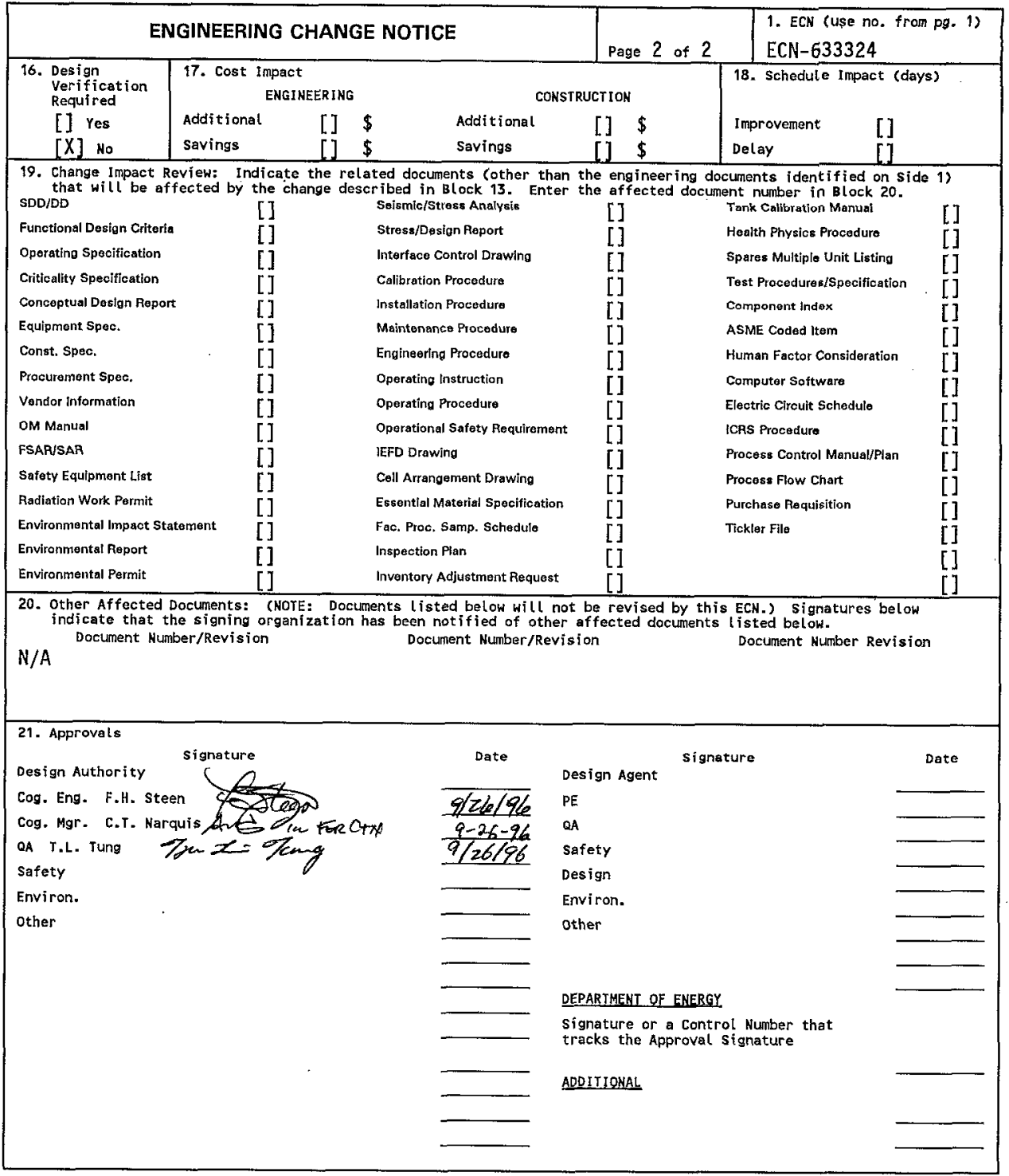




\section{Tank 241-U-106, Cores 147 and 148, Analytical Results for the Final Report}

Franciska H. Steen

Westinghouse Hanford Company, Richland, WA 99352

U.S. Department of Energy Contract DE-AC06-87RL10930

$\begin{array}{lll}\text { EDT/ECN: } & \text { ECN-633324 } & \text { UC: } 2070 \\ \text { Org Code: } & 75725 & \text { Charge Code: MD378 } \\ \text { B\&R Code: } & \text { EW } 3120074 & \text { Total Pages: } 1352\end{array}$

Key Words: Tank 241-U-106, Tank U-106, U-106, U Farm, Core 147, Core 148, Analytical Results, Final Report, Final

Abstract: $N / A$

TRADEMARK DISCLAIMER. Reference herein to any specific comercial product, process, or service by trade name, trademark, manufacturer, or otherwise, does not necessarily constitute or imply its endorsement, recommendation, or favoring by the United states Government or any agency thereof or its contractors or subcontractors.

Printed in the United states of America. To obtain copies of this document, contact: WHC/BCS Document Control Services, P.0. Box 1970, Mailstop H6-08, Richland WA 99352, Phone (509) 372-2420; Fax (509) 376-4989.
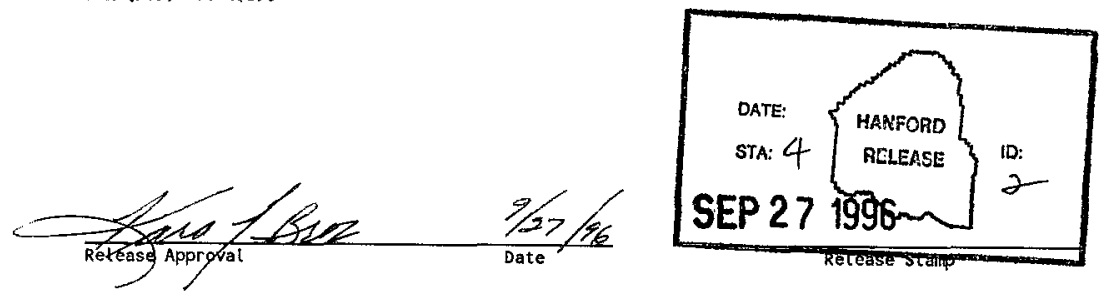

Approved for Public Release 


\section{RECORD OF REVISION}

(1) Document Number

WHC-SD-WM-DP-191

Page 1

(2) Title

Tank 241-U-106, Cores 147 and 148, Analytical Results for the Final Report CHANGE CONTROL RECORD

\begin{tabular}{|c|c|c|c|c|c|}
\hline \multirow{2}{*}{ (3) } & \multirow{2}{*}{ Revision } & \multirow{2}{*}{\multicolumn{2}{|c|}{ (4) Description of Change - Replace, Add, and Delete Pages }} & \multicolumn{2}{|c|}{ Authorized for Release } \\
\hline & & & & (5) Cog. Engr. & (6) Cog. Mgr. \\
\hline & 0 & & $\begin{array}{l}\text { Initially released } 07 / 29 / 96 \text { on EDT- } \\
614800 \text {. }\end{array}$ & F.H. Steen & C.T. Narqujs \\
\hline & & & & teter & For con \\
\hline \multirow{4}{*}{\multicolumn{2}{|c|}{ RS 1}} & & Incorporate per ECN-633324 & FyH. Steen & C.T. Narquis \\
\hline & & & & $4 \leq t+2$ & \\
\hline & & & & $19 / 27 / 96$ & \\
\hline & & & & & \\
\hline & & & & & \\
\hline & & & & & \\
\hline & & & & & \\
\hline & & & & & \\
\hline & & & & & \\
\hline & & & & & \\
\hline & & & & & \\
\hline & & & & & \\
\hline & & & & & \\
\hline & & & & & \\
\hline & & & & & \\
\hline & & & & & \\
\hline & & & & & \\
\hline & & & & & \\
\hline & & & & & \\
\hline & & & & & \\
\hline & & & & & \\
\hline & & & & & \\
\hline & & & & & \\
\hline & & & & & \\
\hline & & & & & \\
\hline & & & & & \\
\hline & & & & & \\
\hline & & & & & \\
\hline & & & & & \\
\hline & & & & & \\
\hline & & & & & \\
\hline & & & & & \\
\hline
\end{tabular}


WHC-SD-WM-DP-191, REV. 1

ANALYTICAL SERVICES

TANK 241-U-106, CORES 147 AND 148 ANALYTICAL RESULTS FOR THE FINAL REPORT

Project Coordinator: FRANCISKA H. STEEN

Prepared for the U.S. Department of Energy office of Environmental Restoration and Waste Management

by

222-S Laboratory

Westinghouse Hanford Company Box 1970

Richland, Washington 
WHC-SD-WM-DP-191, REV. 1

THIS PAGE WAS INTENTIONALLY LEFT BLANK 
WHC-SD-WM-DP-191, REV 1.

TABLE OF CONTENTS

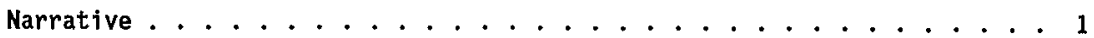

U-106 Sample Breakdown (Attachment 1) . . . . . . . . . . 13

Progagating Reactive System Screening Toot ............. 27

Sample Data Summary . . . . . . . . . . . . . . . 665

Photographs ............................. 111

U-106 Extrusion Reports . . . . . . . . . . . . . 123

Sample Handling . . . . . . . . . . . . . . . . . 136

Extrusion Worklist \# 8675 (2811) . . . . . . . . . . . 138

Extrusion Worklist \# $8676(2812) \ldots \ldots . . . \ldots \ldots 139$

Extrusion Work 1 ist \# $8677(2813) \ldots \ldots . . . . . . . . .140$

Extrusion Work 1 ist \# $8678(2818) \ldots \ldots . . . . . . . . .141$

Extrusion Work 1 ist \# $8679(2919) \ldots \ldots \ldots \ldots . \ldots \ldots$

Extrusion Horklist \#8680 $(2820) \ldots \ldots . . . \ldots \ldots 143$

Extrusion Work list \# $8688(2824) \ldots \ldots . . . \ldots . . . . .144$

Extrusion Workl ist \# $8942(2857) \ldots \ldots . . . \ldots . . . . .145$

Extrusion Workl ist \#8943 $(2858) \ldots \ldots \ldots \ldots$

Sample Preparations . . . . . . . . . . . . . . 147

Fusion Digestion Worklist \# $9468(3079,3080,3083) \ldots \ldots . . .149$

Fusion Digestion Worklist \# $9469 \quad(3081,3084,3082,3085) \ldots . . .150$

Fusion Digestion Worklist \# $9474(3026,3029,3030) \ldots \ldots . . . .152$

Fusion Digestion Work 1 ist \# $9475(3031,3032,3033) \ldots \ldots$

Fusion Digestion Work 1 ist \# $9476(3034,3035) \ldots \ldots \ldots \ldots$

Fusion Digestion Worklist \#10956 $(3878,3884) \therefore \ldots . . .155$

Water Digestion Worklist \# $9471(3093,3094) \ldots \ldots . . \ldots . . .156$

Water Digestion Worklist \# $9472(3095,3096) \ldots \ldots . . . . . .157$

Water Digestion Workl ist \#9473 $(3097,3098,3099) \ldots \ldots . . . .158$

Water Digestion Workl ist \# $9479(3028,3043) \ldots \ldots \ldots . . . .159$

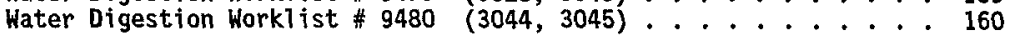

Water Digestion Worklist \# $9481 \quad(3046,3047) \ldots \ldots . . . . . .161$

Water Digestion Workl ist \# $9482(3048,3049) \ldots \ldots . . . . . .162$

Water Digestion Workl ist \# $10958(3879,3880,3885,3886)$. . . 163

Acid Digestion Workl ist \# 9470 (3086, 3087, 3088, 3089, 3090, 3091,

Acid Digestion Worklist \# $9477 \quad 3092)$ (3027, $3036,3037,3038) \cdots \cdots 165$

Acid Digestion Worklist \# $9478 \quad(3039,3040,3041,3042) \therefore \therefore: 168$

Acid Digestion Workl ist \# $10957(3887,3888) \ldots \ldots . . . . .2172$ 
WHC-SD-WM-DP-191, REV 1.

TABLE OF CONTENTS (Continued)

Bulk Density Horkiists . . . . . . . . . . . . . . 173

Inorganic Analyses . . . . . . . . . . . . . 188

Differential Scanning Calorimetry (DSC)

DSC Worklist \# $11662(3876,3882) \ldots \ldots \ldots . . \ldots \ldots$

DSC WorkT ist \# $12418(3876,3882) \ldots \ldots \ldots \ldots \ldots \ldots$

Thermogravimetric Analysis (TGA)

TGA Workl ist \#11655 $(3876,3882) \ldots \ldots . . \ldots 198$

Specific Gravity Analys is ( $S p G)$

SpG Worklist \# $10598(3063,3064) \ldots . . . . . . . . .205$

Cyanide Analysis (CN)

CN Worklist \# 11327 (3071) ................. 213

CN Workl ist \# $11709(3066,3067) \div \ldots 217.1$

CN Workl ist \# $11711(3070,3068,3069) \ldots \ldots . . .218$

CN Workl ist \# $12653(3010,3011,3012,3013) \ldots \ldots \ldots \ldots . . .230$

CN Workl ist \# $12957(3014,3015) \ldots \ldots \ldots \ldots . \ldots 244$

CN Worklist \# $12958(3016,3065) \ldots \ldots \ldots \ldots \ldots . . \ldots 256$

Ion Chromatographic Analys is (IC)

IC Workl ist \# $10597(3063,3064) \ldots \ldots \ldots \ldots . \ldots \ldots$

IC Work1 ist \# $10803(2860,3051) \ldots \ldots \ldots \ldots \ldots \ldots 281$

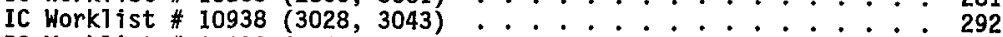

IC Workl ist \# $10939(3044,3045) \ldots \ldots . \ldots . \ldots . \ldots 303$

IC Work] ist \# $10940(3046,3047) \ldots \ldots \ldots . \ldots . \ldots . . \ldots 315$

IC Work jst \# $10941(3093,3094) \ldots \ldots . . \ldots \ldots$

IC Work 1 ist \# $10942(3096,3095) \ldots \ldots . \ldots \ldots$

IC Workl ist \# $10943(3099,3097,3098) \div \ldots$

IC Workl ist \# $12204(3885) \ldots \ldots \ldots \ldots \ldots . . \ldots \ldots$

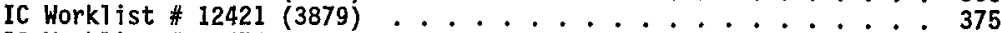

IC Workl ist \# $12474(3048,3049) \ldots \ldots \ldots$

Inductively Coupled Plasma Spectroscopy Analysis (ICP)

ICP Worklist \# $10693(3063,3064) \ldots . . . . . . . . .398$

ICP Workl ist \# $10857(2860,3051) \ldots \ldots$. . . . . . . . 421

ICP WorkT ist \# $10928(3079,3080,3083) \div \ldots 42$

ICP Worklist \# $10929(3081,3082,3084,3085) \div 449$

ICP Workl ist \# $10948(3086,3087,3088,3089,3090,3091,3092): .458$

ICP Worklist \# $10949(3027,3036,3037,3038) \ldots . . . . .2469$

ICP Workl ist \# $10950(3029,3030) \ldots . . . . . . .478$

ICP Workl ist \# $11037(3039,3040,3041,3042) \ldots \ldots . \ldots . . .485$

ICP Workl ist \# $11039(3031,3032,3033) \ldots . . \ldots . . . . .494$

ICP Worklist \# $11042(3034,3035) \ldots \ldots . \ldots . . \ldots 501$

ICP Worklist \# $11838(3026) \ldots \ldots \ldots \ldots . \ldots \ldots$ 
WHC-SD-WM-DP-191, REV 1.

TABLE OF CONTENTS (Continued)

Uranium Analysis (U)

U Worklist \# $12211(3878,3884)$. . . . . . . . . . . . 1129

Part II 45-Day Safety Screening Results for Tank 241-U-106,

Push Mode Cores 147 and 148

Narrative . . . . . . . . . . . . . . . . . . 2-1

U-106 Sample Breakdown (Attachment 1) . . . . . . . . . 2-9

Sample Data Summary . . . . . . . . . . . . . . . . . . . 2-21

Chain of Custody Forms ....................... 2-33

Inorganic Analyses . . . . . . . . . . . . . . . . . 2-45

Differential Scanning Calorimetry (DSC)

DSC Workl ist \# $10567(3067,3070)$. . . . . . . . . . . . 2-47

DSC Horkl ist \# $10568(3065,3066)$. . . . . . . . . . . . . 2-55

DSC Workl ist \# $10569(3063,3064)$. . . . . . . . . . . . . 2-61

DSC Work1 ist \# $10570(3068,3069)$. . . . . . . . . . . . . . 2-67

DSC Workl ist \# $10572(3071)$.................... . . 2-73

DSC Workl jst \# $10765(3063,3064,3071)$. . . . . . . . . . . 2-77

DSC Workl ist \# $10772(2860,3051) . . . . . . . . . . . . . . .2-79$

DSC Workl ist \# $10774(3011)$. . . . . . . . . . . . . . . . 2-87

DSC Workl jst \# $10776(3012,3013)$. . . . . . . . . . . . . . 2-92

DSC Worklist \# $10778(3014,3015)$. . . . . . . . . . . . 2-98

DSC Workl ist \# $10780(3016,3017)$. . . . . . . . . . . . . 2-105

DSC Work1 ist \# $10843(3010)$................... 2-111

DSC Work1 ist \# $10913(3067,3068,3069,3070,3011) . . . . . .2-115$

DSC Workl ist \# $10916(2860,3051,3012,3013,3014,3015,3016$,

$3017)$.................... 2-116

DSC Horklist \# $10930(3010,3065,3066) . . .2 . . .2 . . .2119$

Thermogravimetric Analysis (TGA)

TGA Worklist \# $10574(3067,3070)$. . . . . . . . . . . . 2-121

TGA Worklist \# $10575(3065,3066)$. . . . . . . . . . . . 2-129

TGA Workl ist \# $10576(3063,3064)$. . . . . . . . . . . . 2-135

TGA Worklist \# $10577(3068,3069) \ldots . . . . . . . . . . . .2-141$

TGA Work1 ist \# $10578(3071)$................ 2-149

TGA Workl ist \# $10773(2860,3051)$. . . . . . . . . . . . 2-153

TGA Worklist \# $10775(3010,3011)$. . . . . . . . . . . . . 2-160

TGA Horkl ist \# $10777(3012,3013) \ldots . . . . . . . . . . . .2-168$

TGA Workl ist \# $10779(3014,3015)$. . . . . . . . . . . . . 2-178

TGA Workl ist \#10781 $(3016,3017)$. . . . . . . . . . . . 2-181 
WHC-SD-WM-DP-191, REV 1.

TABLE OF CONTENTS (Continued)

This Document consists of two sections.

Part I consists of pages 1 through 1141, Pages 2, 14, 28, 66, 112, 123, 137, $148,174,189,788,789,790,791,792,793,794,795,796$, and 797 were intenetinally left blank.

Part II consists of pages 2-1 through 2-186 and pages 2-2, 2-10, 2-22, 2-34, and 2-46 were intentionally left blank.

\section{TRADEMARKS:}

Perkin-Elmer is a Registered Trademark of Research and Manufacturing Company, Inc. Mettler is a Registered Trademark of Mettler Electronics 
WHC-SD-WM-DP-191, REV. 1

NARRAT IVE 
WHC-SD-WM-DP-191, REV. 1

THIS PAGE WAS INTENTIONALLY LEFT BLANK 
WHC-SD-WM-DP-191, REV. 1

\section{TANK 241-U-106, CORES 147 AND 148, ANALYTICAL RESULTS FOR THE FINAL REPORT}

This document is the final report deliverable for tank 241-U-106 push mode core segments collected between May 8, 1996 and May 10, 1996 and received by the 222-S Laboratory between May 14, 1996 and May 16, 1996. The segments were subsampled and analyzed in accordance with the Tank 241-U-106 Push Mode Core Sampling and Analysis Plan (TSAP) (Brown, T.M., 1996), the Historical Model Evaluation Data Requirements (Historical DQO) (Simpson, et al., 1995), Data Quality Objective to Support Resolution of the Organic Complexant Safety Issue (Organic DQO) (Turner, et al, 1995) and the Safety Screening Data Quality Objective (DQO) (Dukelow, et al., 1995). The analytical results are included in Table 1.

\section{Sample Handling}

Attachment 1 is a cross reference to relate the tank farm identification numbers to the 222-S Laboratory LABCORE/LIMS sample numbers. These diagrams show the make-up of the core composites and secondary analyses requested. These diagrams and any changes made supersede the diagrams presented in Part II of this document. Sample handling and appearance information for the subsegments can be found in Part II of this document.

Eighteen of the nineteen samples submitted for DSC analysis exceeded the notification limit of 480 Joules/gram (dry weight basis) as stated in the Safety Screening DQO (Dukelow, et al., 1995). Cyanide analysis was requested for these samples, the results are included in this report. Reactive Screening System Tool (RSST) analysis was requested for the sample exhibiting the highest exotherm core 147 segment 5 lower half (S96T004013), results are included in this document.

Two core composites were made, one for each core: 147 and 148. The core composite worksheets are included in this document.

No field blank was provided to the 222-S Laboratory 


\section{WHC-SD-WM-DP-191, REV. 1}

\section{Analytical Results Summary}

The data summary report (Table 1) compiles the analytical results that comply with all applicable DQOs.

\section{Inorganic Analyses}

\section{Differential Scanning Calorimetry (DSC)}

The DSC analyses were performed on the core composite subsamples. The analyses were performed in duplicate on direct subsamples. The exothermic energy based on dry weight of subsample was calculated for all subsamples. The average of the TGA results for each subsample was used in the dry weight correction for that subsample. The results for all DSC analyses and the raw data for the core composite subsamples are included in this report.

The core composite subsamples exceeded the notification limit of 480 Joules/gram (dry weight basis). Appropriate notifications were made. The standard recoveries and RPDs for this analysis were within the required limits.

\section{Thermogravimetric Analysis (TGA)}

The TGA analyses were performed on the core composite subsamples. The analyses were performed in duplicate on direct subsamples. Typically results were determined by summing the weight loss steps which occurred below $200^{\circ} \mathrm{C}$; weight loss steps above this were not used to determine the result. The results for all TGA analyses and the raw data for the core composite subsamples are included in this report.

The standard recoveries and RPDs for this analysis were within the required limits.

\section{Sp.G.-Specific Gravity/Bulk Density}

Specific gravity analyses were performed on the liquid subsamples. The results are provided in Table 1. The raw data are included in this revision. There are no exceptions to the quality control parameters stated in the TSAP for these samples.

Bulk density analyses were performed on direct solid subsamples. The results and raw data for all subsamples are reported in this revision. There are no exceptions to the quality control parameters stated in the TSAP for these samples. 


\section{WHC-SD-WM-DP-191, REV. 1}

\section{Inductively Coupled Plasma Spectrophotometry (ICP)}

The liquid subsamples were prepared for analysis by an acid adjustment of the direct subsample. This is indicated by a "D" in the aliquot class (A\#) column in Table 1. Solid subsamples were prepared for analysis by performing an acid digest as indicated by an " $\mathrm{A}$ " in the A\# column in Table 1 and fusion preparation as indicated by " $F$ " in the A\# column in Table 1.

The QC for all analytes were reviewed for this report. High RPDs were reported for several analytes and were the result of sample inhomogeneity. Reruns were not requested. Table 2 shows a comparison of the result of the sample with that of the serial dilution for those required analytes that had a spike recovery outside the $75 \%-125 \%$ range as set by the Quality Assurance Plan for the 222-S Laboratories (WHC-SD-CP-QAPP-016). Serial dilutions are performed by diluting a sample fivefold. The results from the serial dilution may be found in the raw data and are signified by an "L" at the end of the sample number. For acceptable performance the percent difference between the serial dilution and the undiluted results must be must be $\leq 10$ percent. Five samples had serial dilutions greater than ten percent. This inaccuracy can be attributed to the low concentration of the analyte with respect to the detection limit.

Occasional preparation blanks showed results above the detection level. The levels of these contaminants in the preparation blanks are inconsequential when compared to the result for the sample and do not impact sample data quality. The standard recoveries for this analysis were within the required limits. 
WHC-SD-WM-DP-191 REV. 1

Tablo 2. ICP Serlal Dilution Raw Results for Tank 241-U-106

\begin{tabular}{|c|c|c|c|c|}
\hline Sample ID & Analyte & $\begin{array}{c}\text { Sample Result Undiluted } \\
(U / \sigma)\end{array}$ & $\begin{array}{c}\text { Serial Dilution Result } \\
(\cup g / g)\end{array}$ & \% Difterence \\
\hline S96T002860 & $\mathrm{Al}$ & 11059.46 & 10881.98 & 1.6 \\
\hline$\$ 96 T 002860$ & $\mathrm{~K}$ & 1673.69 & 118.56 & 92.9 \\
\hline $596 T 002860$ & $\mathrm{Na}$ & 211168.33 & $2+9880.28$ & 4.1 \\
\hline$\$ 961002860$ & $\mathrm{~s}$ & 2613.08 & 2732.71 & 4.6 \\
\hline 5961003027 & $\mathrm{Al}$ & 138.90 & 144.70 & 4.2 \\
\hline S967003027 & $\mathrm{G}$ & 1442 & 15.17 & 5.2 \\
\hline 5967003027 & Si & 0.57 & 0.57 & 0.9 \\
\hline S98T003027 & $\mathrm{Na}$ & 710,60 & 737.90 & 3.8 \\
\hline S96T003027 & $\mathbf{s}$ & 10.19 & 10.54 & 3.4 \\
\hline S961003034 & $\mathrm{Na}$ & 446.80 & 444.40 & 0.5 \\
\hline 5961003035 & $\mathrm{Na}$ & 493.00 & 505.50 & 2.5 \\
\hline 5961003036 & Al & 47.30 & 49.15 & 3.9 \\
\hline S961003036 & $\mathrm{P}$ & 7.13 & 7.57 & 6.2 \\
\hline 596T003036 & $\$ i$ & 0.58 & 0.60 & 2.7 \\
\hline S96T003036 & $\mathrm{Na}$ & 696.30 & 721.20 & 3.6 \\
\hline S95T003039 & $\overline{\bar{p}}$ & 12.53 & 12.72 & 1.5 \\
\hline $596 \mathrm{~T} 003039$ & 可 & $0 . \overline{7} \bar{B}$ & 0.85 & 11.3 \\
\hline 5967003030 & $\mathrm{Na}$ & 863.60 & 887.80 & 2.8 \\
\hline S96T003040 & 51 & 0.83 & 0.87 & 4.8 \\
\hline 5961003040 & Na & 909.90 & 928.60 & 2.1 \\
\hline$\$ 961003041$ & AI & 29.65 & 30.63 & 2.6 \\
\hline$\$ 961003041$ & $\overline{\mathrm{C}} \mathrm{r}$ & 6.11 & 6.27 & 2.7 \\
\hline S95T003041 & $\mathrm{Fe}$ & 7.64 & 7.89 & 3.3 \\
\hline S96T003041 & $\mathrm{Mn}$ & 2.29 & 2.35 & 2.7 \\
\hline 5961003041 & $\overline{\mathbf{p}}$ & 8.25 & 8.36 & 1,3 \\
\hline \$967003041 & si & 056 & 0.62 & 19.6 \\
\hline 5961003044 & $\mathrm{Na}$ & 844.70 & 862.90 & $\overline{2.2}$ \\
\hline S96TO03041 & 8 & 7.12 & 7,44 & 4.4 \\
\hline S96T063051 & A) & 13192.22 & 13032.82 & 9.2 \\
\hline S961003051 & $\mathbf{K}$ & 2138.57 & 1366.30 & 36.1 \\
\hline S96T003051 & $\mathrm{Na}$ & 252568.89 & 263667.35 & 4.4 \\
\hline S96T003051 & $\mathrm{s}$ & 3221.42 & 3268.61 & 1.5 \\
\hline 5967003063 & A! & 10725.60 & 10563.47 & 1.5 \\
\hline $596 T 003063$ & $\mathrm{Na}$ & 196648.18 & 207407.75 & 5.5 \\
\hline $596 \mathrm{~T} 003064$ & $\mathrm{Al}$ & 13872.21 & 13557.31 & 23 \\
\hline S96TO03064 & $\overrightarrow{\mathrm{C}}$ & 1125.93 & 1109.64 & 14 \\
\hline 5967003064 & Fe & 847.55 & 833.26 & 1.7 \\
\hline 5967003064 & $\mathrm{Mn}$ & 747.32 & 762.37 & 2.0 \\
\hline 5967003064 & $\mathrm{Na}$ & 234526.67 & 237340.19 & $\overline{1.2}$ \\
\hline S9BT003064 & $\overline{\mathbf{S}}$ & 3181.55 & 3357.72 & $\overline{5} 5$ \\
\hline 596T003083 & $\overrightarrow{\mathrm{Na}}$ & 366.40 & 368.20 & 0.5 \\
\hline 5961003086 & 5 & 3.17 & 2.98 & 6.1 \\
\hline S961003086 & $\mathrm{Na}$ & 897.10 & 253.90 & 4.5 \\
\hline $596 T 003068$ & A) & 59.73 & 62.30 & 4.3 \\
\hline 5967003088 & si & 1.17 & 1.23 & 4.4 \\
\hline $596 \mathrm{~T} 003088$ & $\mathrm{Na}$ & 910.80 & 946.80 & 4.0 \\
\hline $596 \mathrm{~T} 003092$ & $|\vec{A}|$ & 31.25 & 33.04 & 5.7 \\
\hline $596 \mathrm{~T} 003092$ & $F e$ & 31.83 & 33.82 & 6.3 \\
\hline 5967003092 & 51 & 463 & 4.89 & 5.0 \\
\hline $596 \mathrm{TO0} 3092$ & $\mathrm{Na}$ & 994.00 & 1055.00 & 6.1 \\
\hline 5967003880 & Al & 41.97 & 42.38 & 1.0 \\
\hline S96T0123830 & $\mathrm{Na}$ & 919.90 & 932.30 & 1.3 \\
\hline $596 \mathrm{~T} 003886$ & $\mathrm{Na}$ & 961.90 & 971.30 & 1.0 \\
\hline $596 T 003887$ & Al & 57.24 & 59.97 & 48 \\
\hline 5967003897 & Fe & 18.30 & 19.24 & 51 \\
\hline S967003687 & $\mathrm{Mn}$ & 7.35 & 7.68 & 4.5 \\
\hline 5967003887 & $\mathrm{P}$ & 1764 & 18.56 & 5.8 \\
\hline 5965003887 & $\mathrm{Na}$ & 85 & 697.90 & 4.6 \\
\hline 5967003888 & A) & 61.02 & 62.53 & 2.5 \\
\hline 5967003888 & Fe & 12.85 & 13.31 & 3.6 \\
\hline 596,003888 & $\mathrm{Mn}$ & 4.41 & 4.54 & 2.9 \\
\hline 5967003888 & $\overline{\mathbf{P}}$ & 12.99 & 1360 & 47 \\
\hline 596 T00 3898 & An & 0.08 & 0,07 & 22.2 \\
\hline
\end{tabular}

$*$ Diference $=($ ABS(Samplo - Sarlaf $)) /$ Sample $\times 100$ 


\section{WHC-SD-WM-DP-191, REV. 1}

\section{Ion Chromatography (IC)}

The IC analysis was performed on direct subsamples of liquid samples, as indicated by a blank in the A\# column in Table 1. The solid subsamples were prepared for analyses by performing a water digest. This is indicated with a "W" in the A\# column in Table 1.

High RPDs were reported for three of the subsamples submitted for analysis. These high RPDs were attributed to sample inhomogeneity. Additional reruns would not significantly improve the results due to the difficulty in obtaining representative samples of the material. Six subsamples had spike recoveries outside of the $75 \%$ to $125 \%$ range on several of the anions. No reruns were requested.

Occasional preparation blanks for chloride and nitrate showed results above the detection level. The levels of these analytes in the preparation blank are inconsequential when compared to the result for the sample. This contamination does not impact sample data quality. The standard recoveries for this analysis were within the required limits.

\section{TIC/TOC - Total Inorganic/Organic Carbon Persulfate}

Total Inorganic Carbon (TIC) and Total Organic Carbon (TOC) by persulfate/coulometry analyses were performed in duplicate on direct subsamples. Low concentrations of TIC and TOC were noted in some preparation blanks, however the levels of contamination are inconsequential when compared to the result of the sample and do not impact sample data quality. A high RPDs was reported for one subsample. This was due to sample inhomogeneity. A rerun was not requested. The standard recoveries and spike recoveries for these analyses were within the required limits.

\section{TOC - Total Organic Carbon By Furnace Oxidation}

TOC by furnace oxidation analysis was requested as a secondary analysis for the samples that exceeded the DSC notification limits and the TOC by persulfate/coulometry did not account for the high exotherms. This analysis was performed on the four direct liquid subsamples. Low concentrations of TOC were noted in some preparation blanks, however the level is inconsequential when compared to the result of the sample. This contamination does not impact sample data quality. The standard recoveries, spike recoveries and RPDs for this analysis were within the required limits.

\section{$\mathrm{CN}$ - Cyanide}

Cyanide was requested as a secondary analysis due to the high energetics in the tank samples. 


\section{WHC-SD-WM-DP-191, REV. 1}

With the subsequent changes to the Organic DQO, the cyanide analyses would not have been required therefore the results are presented in this report as "opportunistic analyses". The cyanide by water method is for the determination of total cyanide in samples that contain only soluble cyanide compounds. The cyanide results ranged from less than $4.23 \mu \mathrm{g} / \mathrm{g}$ to $35.1 \mu \mathrm{g} / \mathrm{g}$. The standard recoveries. spike recoveries and RPDs for these analyses were within the required limits.

\section{Radionuclide Analyses}

\section{AT - Total Alpha}

Total alpha activity (AT) analysis was performed on the solid core composite subsamples. The samples were prepared for analysis by performing a fusion digest in duplicate. The fusion preparation is indicated with an " $F$ " in the A\# column in Table 1. The results and the raw data for all subsamples are included in this revision. A high RPD was reported for sample S96T003884 and was due to sample inhomogeneity. No rerun was requested. The standard recoveries and spike recoveries for this analysis were within the required limits.

\section{TB - Total Beta}

Total beta activity (TB) was performed on the solid core composite subsamples. The samples were prepared for analysis by performing a fusion digest in duplicate. The fusion preparation is indicated with an " $F$ " in A\# column in Table 1. A high RPD was reported for sample S96T003884 and was due to sample inhomogeneity. No rerun was requested. The standard recoveries and spike recoveries for this analysis were within the required limits.

\section{${ }^{9 n} \mathrm{Sr}$ - Strontium 90}

${ }^{90} \mathrm{Sr}$ analysis was performed on the solid core composite subsamples. The samples were prepared for analysis by performing a fusion digest in duplicate. The fusion preparation is indicated with an " $F$ " in the A\# column in Table 1. The preparation blanks showed results for strontium-90 slightly above the detection level. The levels in the preparation blanks are inconsequential when compared to the results for the samples. These contaminants do not impact sample data quality. A high RPD was reported for sample S96T003884 and was due to sample inhomogeneity. No rerun was requested. The standard recoveries for this analysis were within the required limits.

\section{GEA - Gamma Energy Analysis}

GEA analysis was performed on the solid subsamples. The samples were prepared for analysis by performing a fusion digest in duplicate. The fusion preparation is indicated with an " $F$ " in the 


\section{WHC-SD-WM-DP-191, REV. 1}

A\# column in Table 1 .

Only the QC for required analytes $\left({ }^{137} \mathrm{Cs}\right)$ were considered in this report. Other "opportunistic" analytes are included but were not reviewed for QC. High RPDs were reported for samples S96T003079 and S96T003884. This was due to sample inhomogeneity and no rerun were requested. The standard recoveries for this analysis were within the required limits.

Actual detection limits for GEA analytes are not currently available. The latest GEA software does not report a minimum detectable activity (MDA). If an analyte is reported as "less than", the value reported is the detection limit.

\section{U - Total Uranium}

Uranium analysis was performed on the core composite subsamples. The samples were prepared for analysis by performing a fusion digest in duplicate. The fusion preparation is indicated with an " $F$ " in the A\# column in Table 1. The standard recoveries, spike recoveries and RPDs for this analysis were within the required limits. 
WHC-SD-WM-DP-191, REV. 1

\section{Procedures}

Table 3 lists the analytical procedures used for performing the sample analyses. Abbreviations for analyses are defined in the table notes.

Table 3. Analytical Procedures

\begin{tabular}{|c|c|c|c|}
\hline Analysis & $\begin{array}{l}\text { Sample } \\
\text { Portion }\end{array}$ & $\begin{array}{l}\text { Preparation } \\
\text { Procedure + }\end{array}$ & $\begin{array}{l}\text { Analysis } \\
\text { Procedure }\end{array}$ \\
\hline DSC & Solid/Liquid & $\mathrm{N} / \mathrm{A}$ & $\begin{array}{l}\text { LA-514-114 Rev. C-1 } \\
\text { LA-514-113 Rev. C-1 }\end{array}$ \\
\hline TGA & Solid/Liqquid & $\mathrm{N} / \mathrm{A}$ & $\begin{array}{l}\text { LA-514-114 Rev, C-1 } \\
\text { LA-560-112 Rev. B-1 }\end{array}$ \\
\hline Bulk Density & Solid & $\mathrm{N} / \mathrm{A}$ & LO-160-103 Rev. B-0 \\
\hline Sp.G. & Liquid & N/A & LA-510-112 Rev. C-3 \\
\hline $\mathrm{CN}$ & $\begin{array}{l}\text { Liquid } \\
\text { Solid }\end{array}$ & $\mathrm{N} / \mathrm{A}$ & $\begin{array}{l}\text { LA-695-102 Rev. A-0 } \\
\text { LA-695-103 Rev. A-0 }\end{array}$ \\
\hline IC & $\begin{array}{l}\text { Solid } \\
\text { Liquid }\end{array}$ & $\begin{array}{l}\text { LA-504-101 Rev. E-0 } \\
\text { N/A }\end{array}$ & LA-533-105 Rev. D-1 \\
\hline ICP & $\begin{array}{l}\text { Solid } \\
\text { Liquid }\end{array}$ & $\begin{array}{l}\text { LA-505-159 Rev. D-0 } \\
\text { LA-549-101 Rev. F-0 } \\
\text { LA-504-101 Rev. E-0 } \\
\text { N/A }\end{array}$ & $\begin{array}{l}\text { LA-505-151 Rev. D-3 } \\
\text { LA-505-161 Rev. B-1 }\end{array}$ \\
\hline TICTOC & $\begin{array}{l}\text { Liquid } \\
\text { Solid }\end{array}$ & $\mathrm{N} / \mathrm{A}$ & LA-342-100 Rev. D-0 \\
\hline TOC & Liquid & $\mathrm{N} / \mathrm{A}$ & LA-344-105 Rev. D-1 \\
\hline
\end{tabular}


WHC-SD-WM-DP-191, REV. 1

\begin{tabular}{|c|c|c|c|}
\hline AT/TB & Solid & LA-549-101 Rev. F-0 & LA-508-101 Rev. D-2 \\
\hline GEA & Solid & LA-549-101 Rev. F 0 & LA-548-121 Rev. E-0 \\
\hline${ }^{90} \mathrm{Sr}$ & Solid & LA-549-141 Rev. F-0 & LA-220-101 Rev. D-1 \\
\hline U & Solid & LA-549-101 Rev. F-0 & LA-925-009 Rev. A-1 \\
\hline
\end{tabular}

Abbreviations:

N/A = not applicable (these are direct samples)

DSC = differential scanning calorimetry

TGA = thermogravimetric analysis

Sp.G, = specific gravity

$\mathrm{CN}=$ cyanide

$\%$ Water = percent water by gravimetric analysis

IC = ion chromatography

ICP = inductively coupled plasma

TOC = total organic carbon

TIC = total inorganic carbon

AT = total alpha

TB = total beta

$\mathrm{GEA}=$ gamma energy analysis

${ }^{137} \mathrm{Cs}=$ cesium 137

${ }^{*} \mathrm{Sr}=$ strontium 90

U = uranium 


\section{WHC-SD-WM-DP-191, REV. 1}

\section{References}

Brown, T. M., 1996, Tank 241-U-106 Push Mode Core Sampling and Analysis Plan, WHC-SD-WM-TSAP-093, Rev. 0, Westinghouse Hanford Company, Richland, WA 99352.

Dukelow, G. T.. J. W. Hunt, H. Babad, and J. E. Meacham, 1995, Tank Safety Screening Data Quality Objective, WHC-SD-WM-SP-004, Rev. 2, Westinghouse Hanford

Company, $\quad$ Richland, WA 99352

Simpson, B. C., and D. J. McCain, 1995, Historical Model Evaluation Data Requirements, WHC-SD-WM-DQO-018, Rev. 0, Westinghouse Hanford Company, Richland, WA 99352.

Turner, D. A., H. Babad, L. L. Buckley, and J. E. Meacham, 1995, Data Quality Objective to Support Resolution of the Organic Complexant Safety Issue, WHC-SD-WM-DQO-006, Rev. 2, Westinghouse Hanford Company, Richland, WA 99352. 
WHC-SD-HM-DP-191, REV. 1

ATTACHMENT 1

U-106 SAMPLE BREAKDOWN 
WHC-SD-WM-DP-191, REV . 1

THIS PAGE WAS INTENTIONALLY LEFT BLANK 


\section{WHC-SD-WM-DP-191, REV.I}

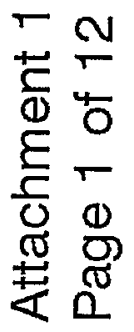

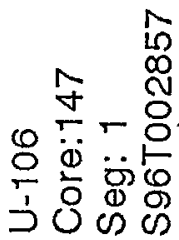

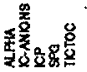

品正

— $13 \frac{8}{8}$

10

吅喜

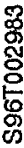

亮 $\frac{0}{5}$

5
8
8
8
8

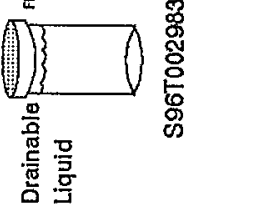




\section{WHC-SD-WM-DP-191, REV.I}

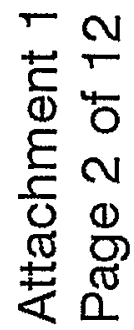

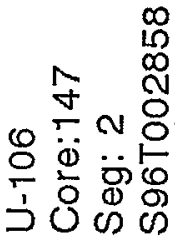

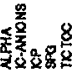

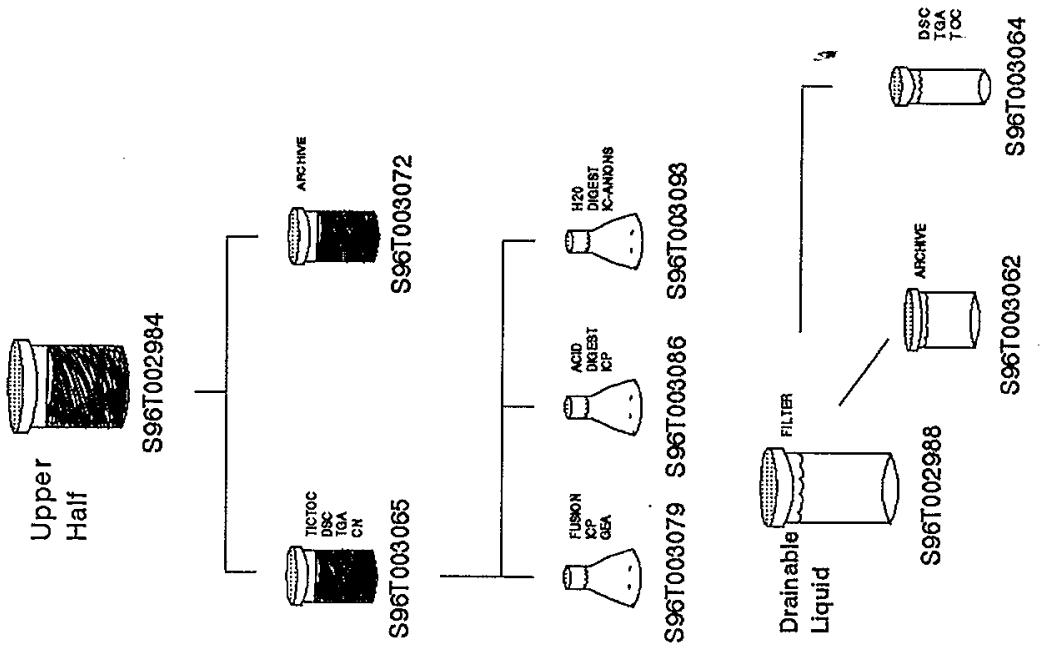


This page cannot be converted.

Please view the native document

for the original page. 

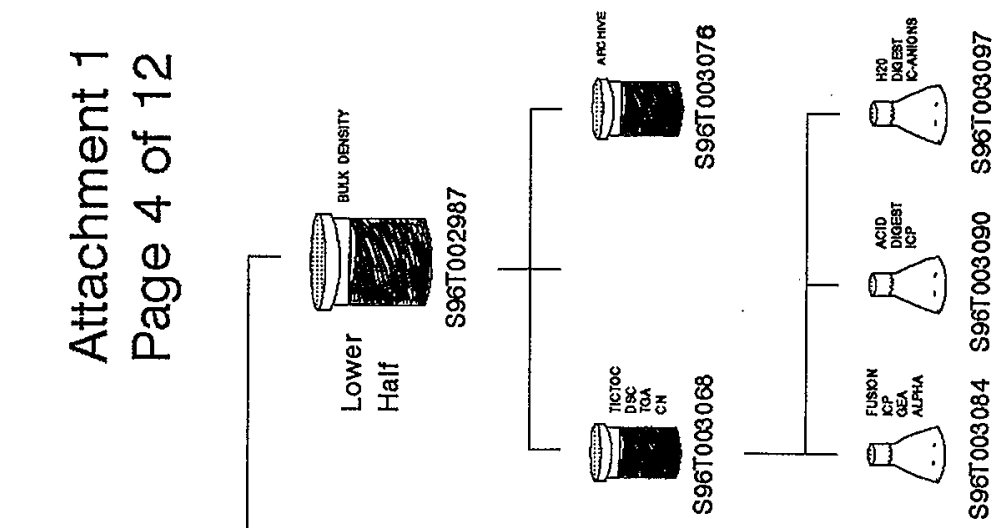

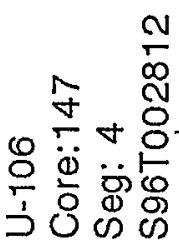

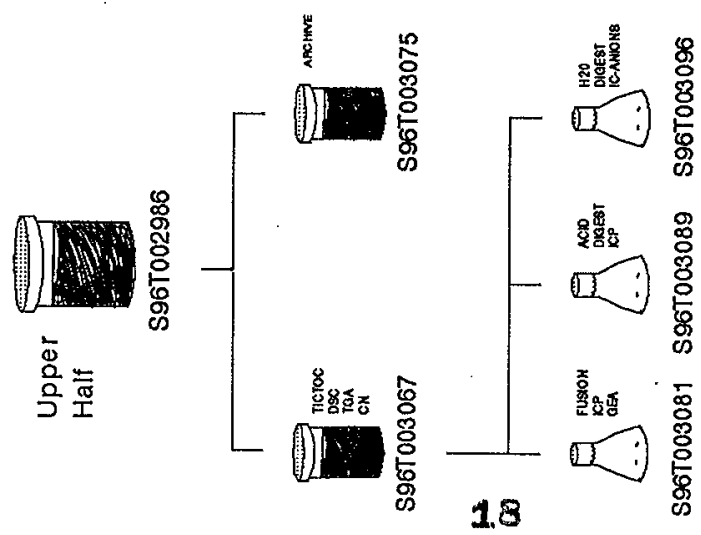


WHC-SD-WM-DP-191, REV.I

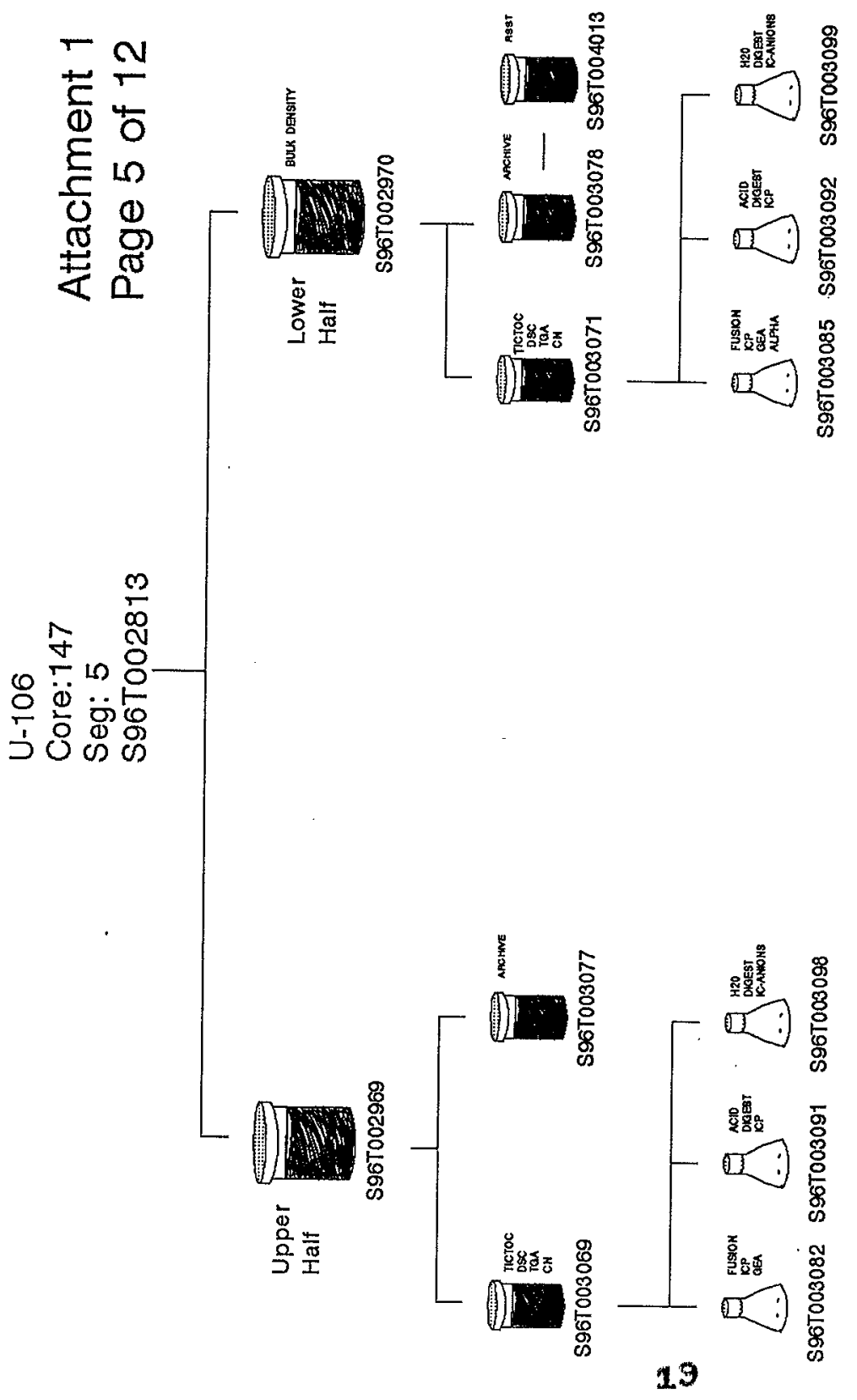



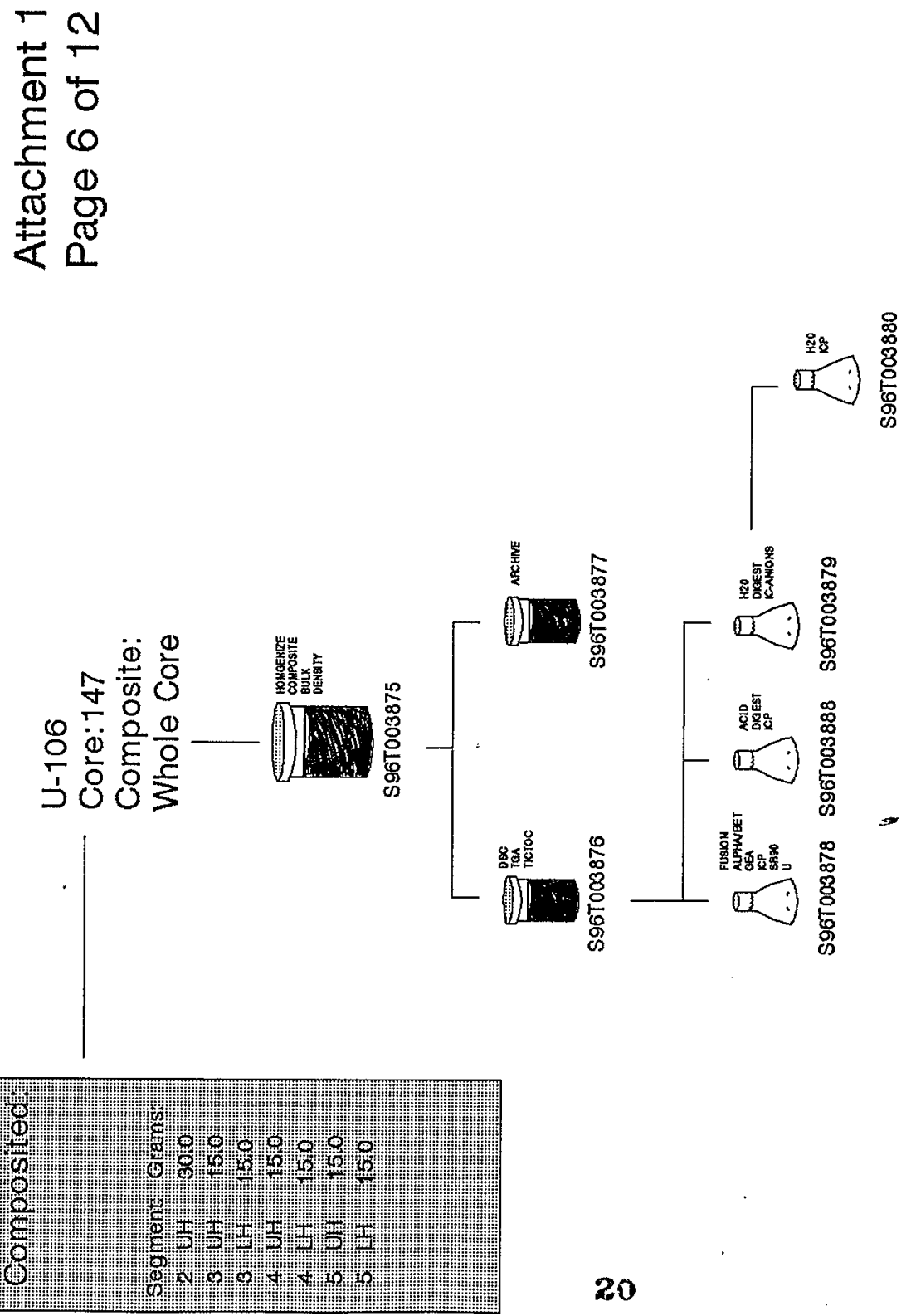
WHC-SD-WM-DP-191, REV.I
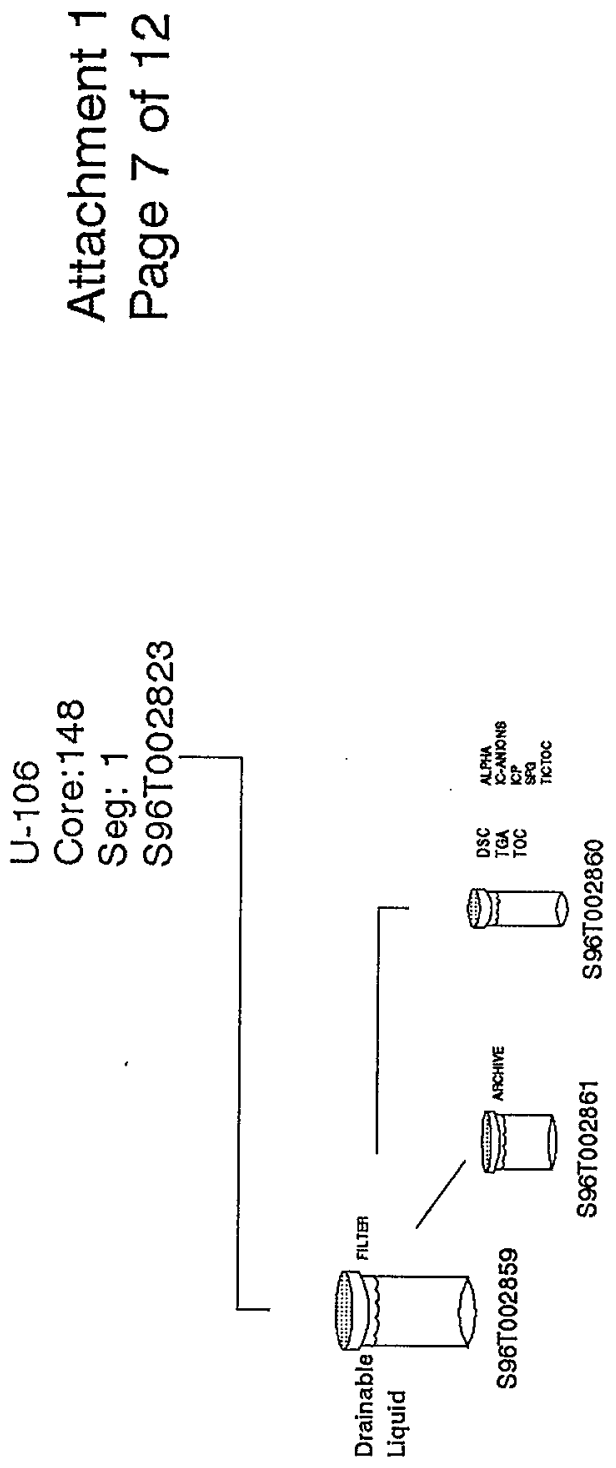

21 
WHG-SD-WM-DP-191, REV. I

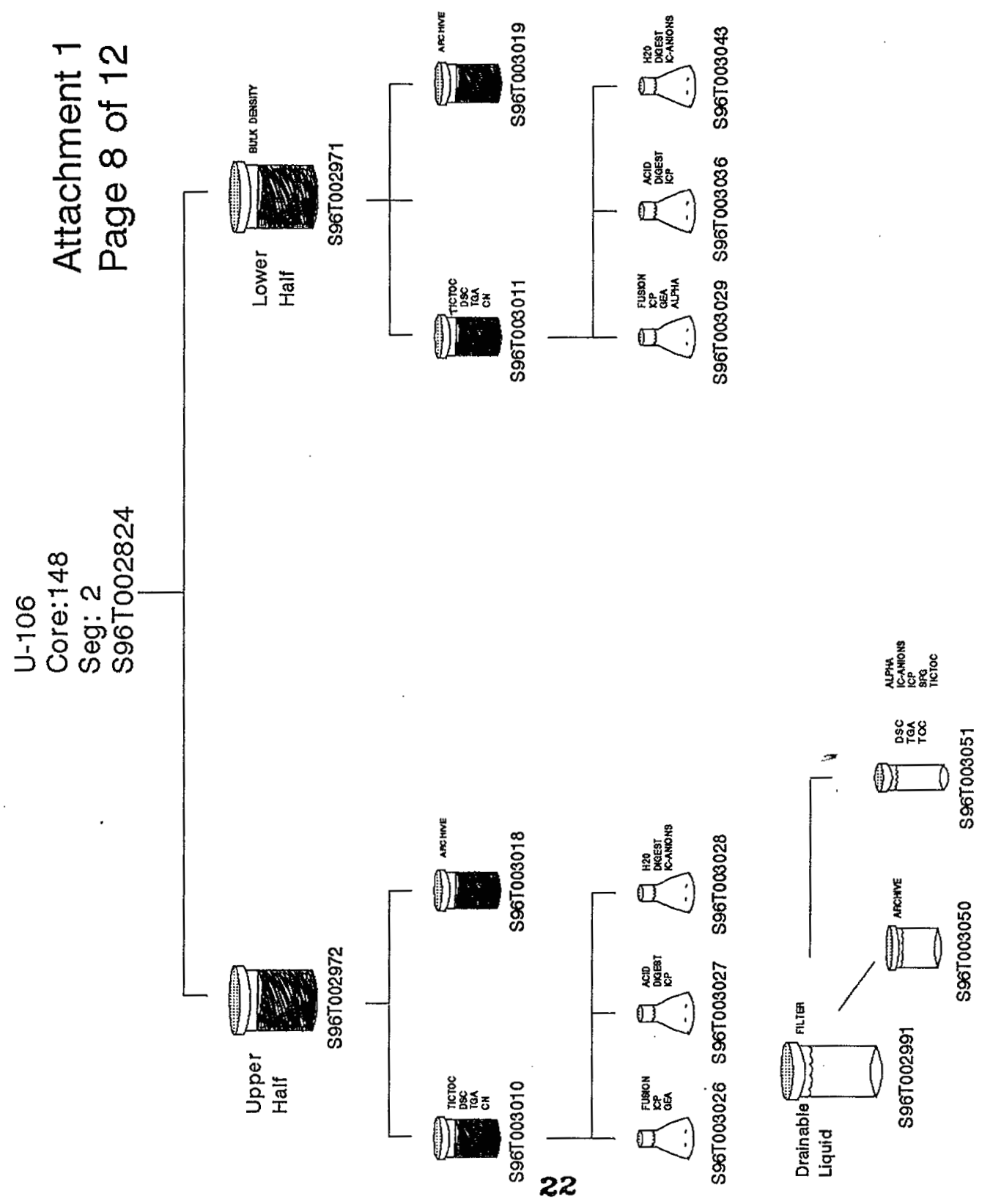


WHC-SD-WM-DP-191, REV. I

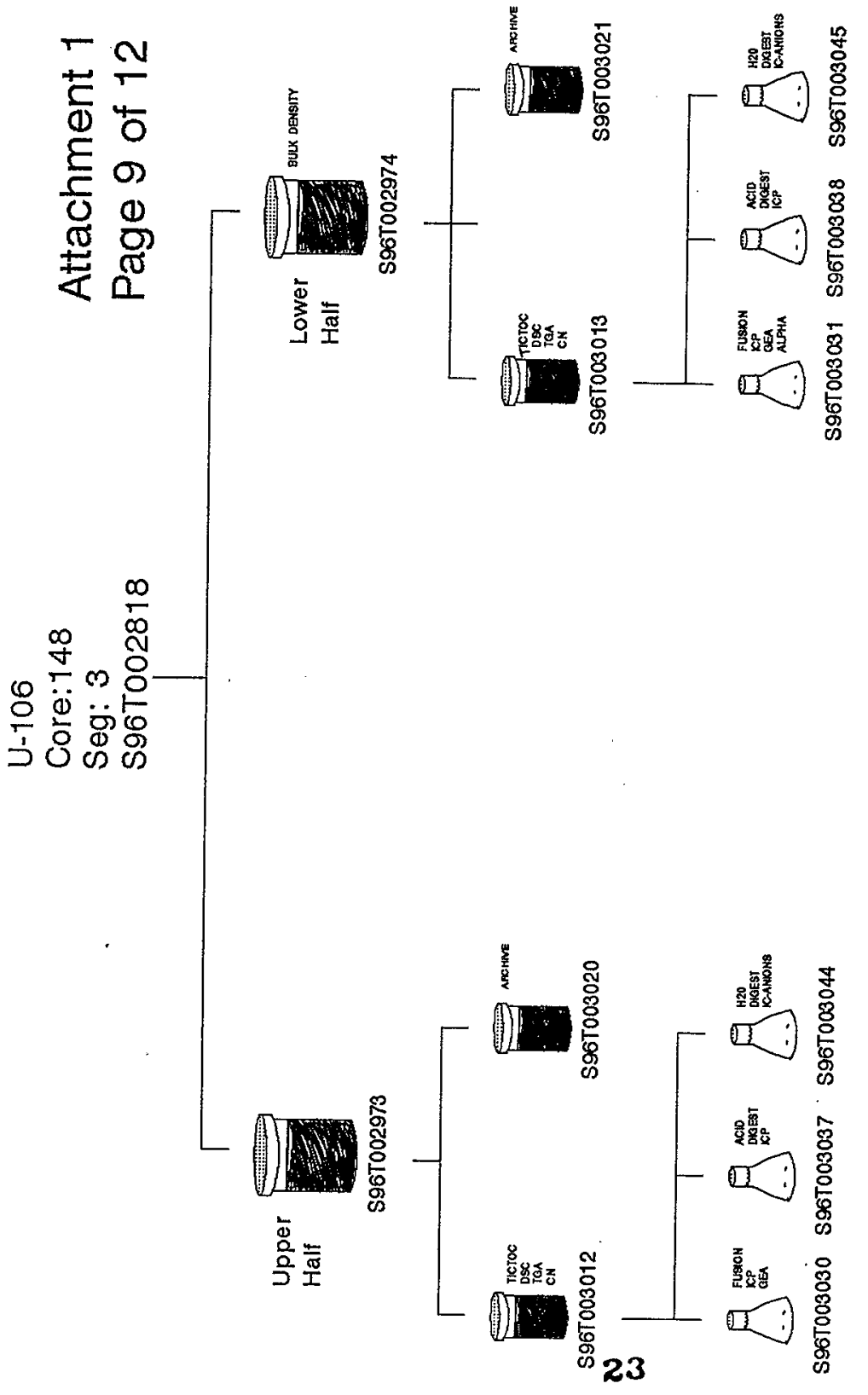




\section{WHC-SD-WM-DP-191, REV.I}

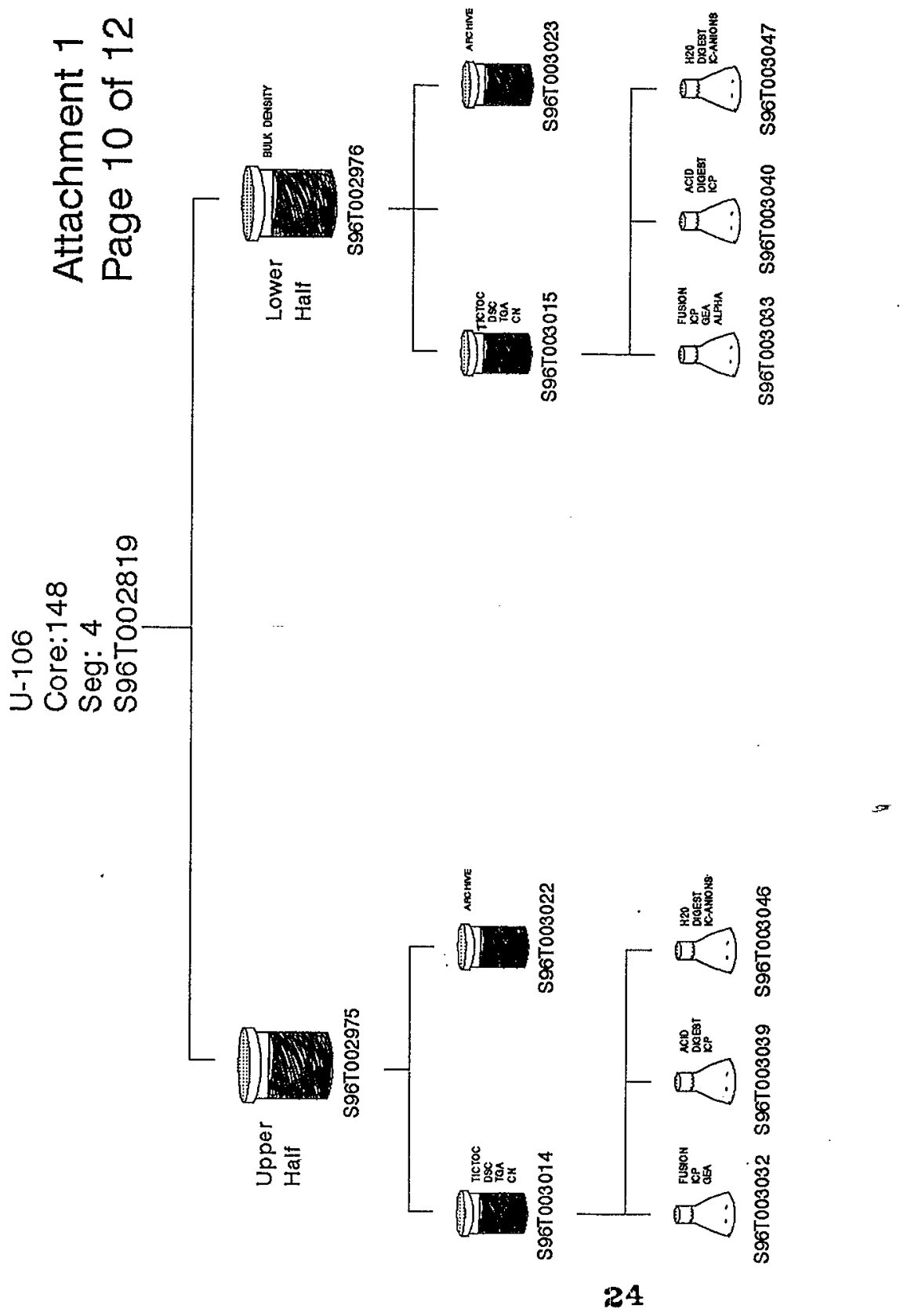


WHC-SD-WM-DP-191, REV.I

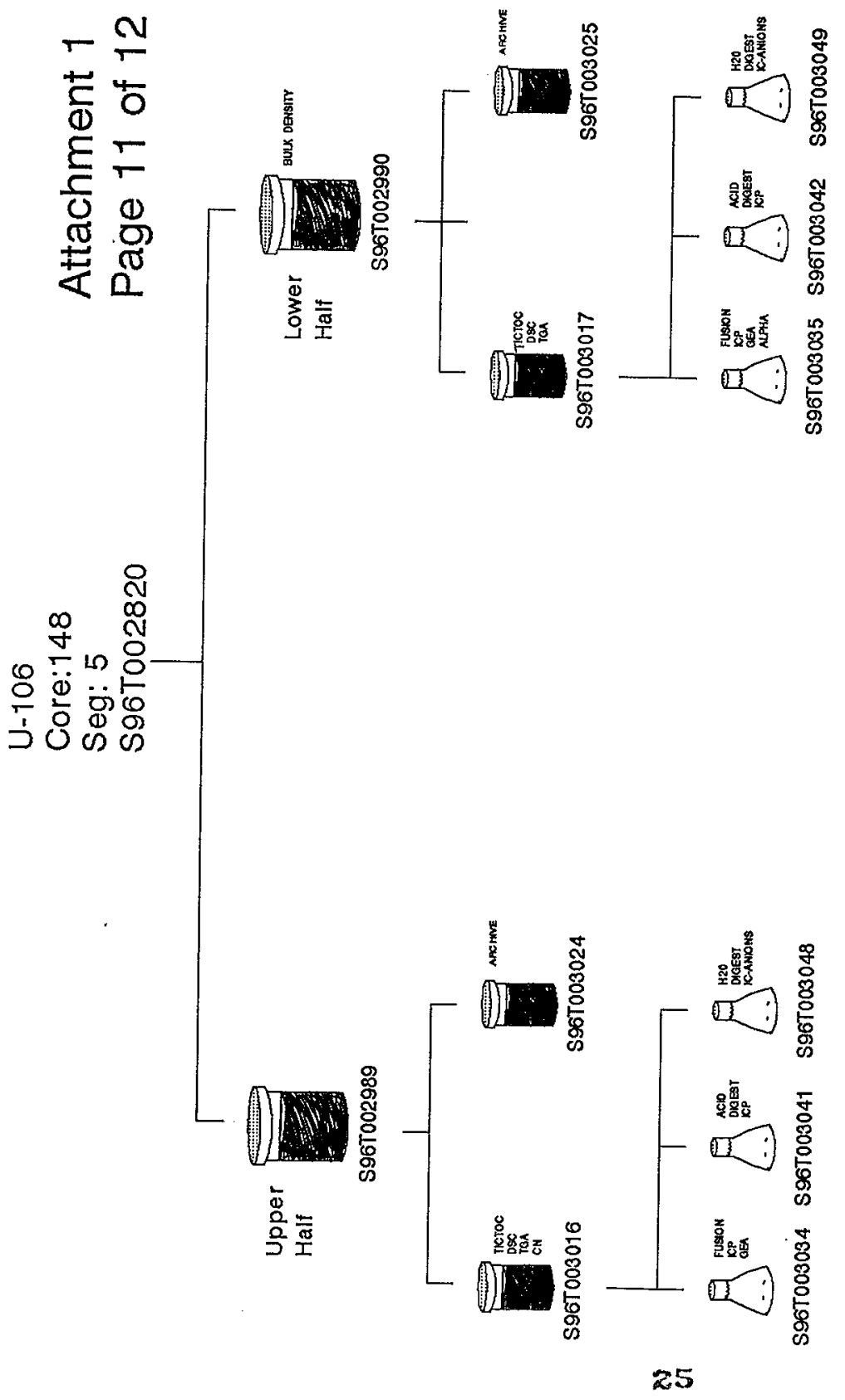




\section{WHC-SD-WM-DP-191, REV.I}

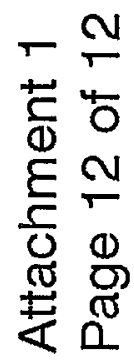

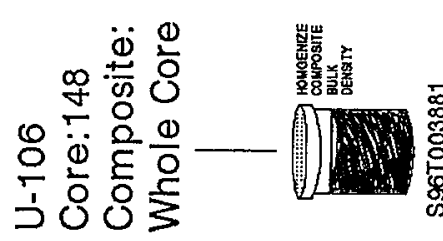
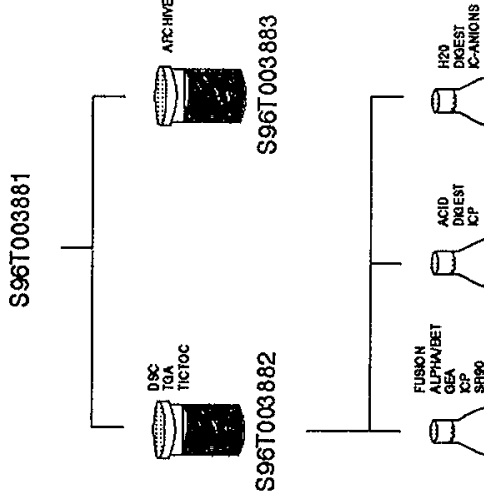

\begin{tabular}{l}
4 \\
$\infty$ \\
$\infty$ \\
8 \\
0 \\
\hline 8 \\
8 \\
5 \\
$\infty$ \\
8 \\
8 \\
6 \\
8 \\
0
\end{tabular}

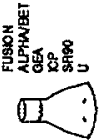

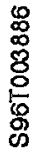

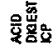<smiles>C1=CC2C=CC1C2</smiles>

\pm
8
8
8
$\frac{8}{8}$
8

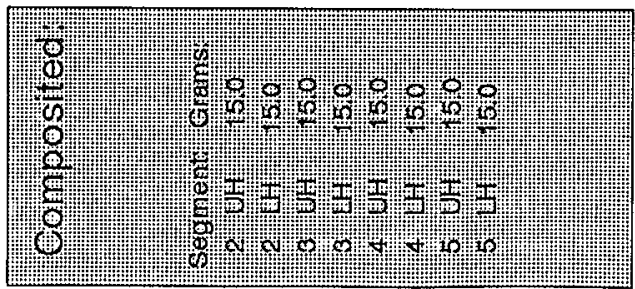


WHC-SD-WM-DP-191, REV. 1

PROGAGATING REACTIVE SYSTEM SCREENING TOOL 
HHC-SD-WM-DP-191, REV. 1

THIS PAGE WAS INTENTIONALLY LEFT BLANK 
From: Process-Chemistry \& Statistics

Phone: 373-2162

75764-PCS96-091

Date: $\quad$ September 9, 1996

Subject: RSST ADIABATIC CALORIMETRY OF U-106 SLUDGE SAMPLE

To:

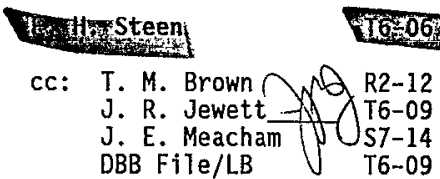

\section{SUMMARY/CONCLUSIONS}

Sample S96T004013 from segment 3 of a U-106 core was dried over low heat to remove $17.4 \%$ moisture, then size-reduced for a total of two complete RSST tests. Testing at approx. 7 barg overpressure yjelded weak self-heat events, starting at $210^{\circ} \mathrm{C}$ to $225^{\circ} \mathrm{C}$, and ending at a maximum of $400^{\circ} \mathrm{C}$, to give a maximum $\Delta T$ of $119 \phi^{\circ} \mathrm{C}$, ( $\phi$ is defined below), a maximum self-heat rate of $42^{\circ} \mathrm{C} / \mathrm{min}$, and a maximum $1.01 \times 10^{-3}$ mole/gram of noncondensible gas generation in duplicate testing. The maximum derived estimator of energy yield is given by $158 \times$ (specific heat capacity) $+16 \mathrm{~J} / \mathrm{g}^{\mathrm{dry}}$.

\section{PERFORMANCE OF WORK}

Work was performed according to WHC-SD-WM-TP--104, and calculations (attachments 1 to 3 ) were made as outlined in WHC-SD-WM-DTR-026. Sample treatment and experimental data were logged in WHC-N-1014-1, pg. 78-85, and separate hardcopies of instrumental data were retained in WHC-N-1376-1 to back up electronic copies.

\section{SAMPLE TREATMENT}

There was one sample submitted for RSST analysis from the U-106 tank. Table 1 identifies the tests based on this sample and reports the various states of moisture/volatiles loss it experienced. 
F. H. Steen

75764-PCS96-091

Page 2

September 9, 1996 .

Table 1. Sample Identification and Percent Volatiles From As-Received to Various States

\begin{tabular}{|c|c|c|c|c|}
\hline Test ID & Sample & $\begin{array}{c}\% \mathrm{H}_{2} \mathrm{O} \text { Lost to } \\
\text { Prepared } \\
\text { State }\end{array}$ & $\begin{array}{l}\% \mathrm{H}_{2} \mathrm{O} \text { Lost to } \\
\text { Dry State }\end{array}$ & $\begin{array}{l}\text { \%Total } \\
\text { Volatiles } \\
\text { Lost to } \\
\text { Reacted } \\
\text { State }\end{array}$ \\
\hline 960815 & $\begin{array}{c}\text { S96T004013 } \\
\text { - Vial } 10696 \\
\text { U-106 S2 }\end{array}$ & 17.47 & 44.57 & $\begin{array}{l}\text { Canceled } \\
\text { heatup } \\
\text {-See below }\end{array}$ \\
\hline 960819 & $\begin{array}{l}\text { Reheat of } \\
\text { above to } \\
\text { complete the } \\
\text { test }\end{array}$ & See above & See above & 24.73 \\
\hline 960826 & $\begin{array}{c}\text { New al iquot } \\
\text { of same }\end{array}$ & See above & See above & 26.31 \\
\hline
\end{tabular}

a Weight loss from as-received to as-prepared for RSST.

b Weight 70 s by $222-51$ ab reported results for as-delivered to as-dry -the dry weight basis.

' Calculated from loss on reaction in RSST and loss on preparation. Usually low due to condensate on sample insulation sheath.

As received, the sample was a wet, sticky material that needed to be dried and size-reduced in order to make it suitable for RSST analysis. This is done for two reasons:

a) To be able to get the sample into the RSST sample vesse1, and

b) To prevent the evolution of large amounts of water, which creates sample voids, tends to push sample out of the sample holder, and compromises the thermal insulation around the holder.

The sample was dried in a beaker sitting on a hot plate at low heat for two days. After having been dried, it was ground up for dispensing into the RSST holder. Loaded samples were run in the RSST under approximately 7 barg nitrogen (assured by first flushing the bomb with nitrogen twice before a final fill). The selected heating rate for all runs was approximately $1^{\circ} \mathrm{C} / \min$.

During Test 960815 , it was noted that the heater circuit had suffered a blown fuse, and so the run was canceled at approximately $212^{\circ} \mathrm{C}$ before any self-heating was apparent. After repairs, the same sample was rerun through a complete heating program in Test 960819 . Another aliquot of prepared sample was run in Test 960826 to provide a duplicate experiment to verify the results obtained in the two previous tests. 
F. H. Steen

75764-PC\$96-091

Page 3

September 9, 1996

\section{THERMAL RESULTS}

\section{Test 960815}

Figure 1 (attachment 1) il Tustrates the thermal response from the abortive test 960815 , and these results are summarized in Table 3 . During the heatup, the slow heat rate achieved indicated a partial heater failure that was subsequently found to be a blown fuse, so the run was canceled at a temperature of approximately $212^{\circ} \mathrm{C}$. No self-heating response was evident before the run was canceled, therefore the sample was viewed as intact and available for a reheat when repairs could be made to the RSST heater circuit. The sample aliquot was left sealed up and undisturbed in the RSST bomb.

\section{Iest 960819}

The sample aliquot from the previous test was reheated in this run. The results are 11 lustrated in Figures 1 and 2 (attachment 2) and are reported in TabTe 3. Between approximately $150^{\circ} \mathrm{C}$ and $225^{\circ} \mathrm{C}$, the instrumentally driven heat rate was perturbed and depressed by passage of the sample through an endothermic regime. By $225^{\circ} \mathrm{C}$, though, it was clear that the sample had finished traversing this regime and was contributing internally generated heat. The heat rate gyrated somewhat, exciting more heater offset, but finally attained a weak $42{ }^{\circ} \mathrm{C} / \mathrm{min}$ rate before maximum heater compensating power and apparent reactant exhaustion at $340^{\circ} \mathrm{C}$.

The general shape of the temperature rate curve in Figure 2 (attachment 2) is more complex than would be obtained from a simple first order reaction. The hesitation and acceleration occurring at low self-heat rates could possibly be due to the melt of more oxidizer, consequently followed by increased oxidation rates. The sample in test 960819 did not show any indication of propagation.

The initial stages of the complex self-heating event (where changes in reactant stoichiometry are not yet significant) were crudely, collectively fitted by Arrhenius parameters and a fixed value of baseline heating rate. The baseline value of $0.81^{\circ} \mathrm{C} / \mathrm{min}$ was determined by fitting baseline data at temperatures below the endothermic regime. These parameters are listed in Table 3.

The $\phi$ factor appearing in the tables, and defined by the footnote, removes the effects of instrument heat capacity from the initial stage and fina? state results. It is by definition always greater than unity, but is likely to be less than 1.09 in value. To estimate its value, one needs to provide an estimate of the average heat capacity that the sample possesses while the exothermic reaction is occurring because, unfortunately, a measured value is not currently available.

The exothermic energy content of the sample could be calculated (attachment 2) from the baseline-corrected temperature change if an estimated average 
F. H. Steen

Page 4

September 9, 1996.

reacting heat capacity were available. The formula that would be used would be:

$$
\hat{Q}_{d r y}=85.8\left(\bar{C}_{\mathrm{ps}}^{\text {react }}\right)+7.45 \frac{\mathrm{s}}{\mathrm{g}^{\mathrm{dry}}}
$$

This is provided for the convenience of those willing to venture a heat capacity estimate in order to obtain an estimate of specific energy release.

\section{Iest 960826}

Figures 1 and 2 (attachment 3 ) comprise the thermal response resuTts for this test. The sample began to self-heat at $210^{\circ} \mathrm{C}$, traversing several weak self-heating regimes before attaining a maximum $14^{\circ} \mathrm{C} / \mathrm{min}$, then exhausting itself at $400^{\circ} \mathrm{C}$. The events observed were in approximate agreement with DSC thermograms taken from wet. samples (S96T003071).

The initial self-heating responses were collectively and crudely fitted by Arrhenius parameters reported in Table 3 . For this test the value of $\phi$ is unlikely to have exceeded 1.1 .

The energy released from the sample in this test would be estimated by:

$$
\hat{Q}_{d x y}=158.2\left(\bar{C}_{\mathrm{ps}}^{\text {resct }}\right)+16 \frac{\mathrm{J}}{\mathrm{g}^{\mathrm{dry}}}
$$

if an average reacting specific heat capacity were available. The average of equations (1) and (2) would be:

$$
\text { Mean } \hat{Q}_{\mathrm{dry}}=(122.0 \pm 51)\left(\overline{\mathrm{C}}_{\mathrm{ps}}^{\mathrm{xeact}}\right)+(11.7 \pm 6) \frac{\mathrm{J}}{\mathrm{g}^{d x y}}
$$

\section{GAS PRODUCTION RESULTS}

\section{Test 960815}

Pressure evolution data and gas production calculation results appear in Figures 1 through 3 (attachment 1) and Tables 2 and 4 below. (Note that the dry-weight-basis results reported in the table are based on TGA results on original samples from the core segment, not on RSST sample preparation results). Figure 3 (attachment 1) was calculated from the instrumental data as suggested in the footnotes to Table 2 (see WHC-SD-WM-DTR-026). Clearly some noncondensible gas was generated by non-exothermic decomposition during this test. Table 4 compares these calculations with those based on the auxiliary data in Table 2 . 
F. H. Steen

75764-PCS96-091

Page 5

September 9, 1996.

\section{Test 960819}

Relevant results appear in Figures 1,3 and 4 (attachment 2) and Tables 2 and 4 below. It should be pointed out that the calculations which yield Figure 4 (attachment 2) are based on near-steady-state temperature distributions in the RSST bomb, and give erroneous results when temperatures rise or fall rapidly. Nevertheless, Table 4 indicates that more gas was generated in this reheat test.

The total gas generated in the aliquot used in the first two tests is the sum of each individual test. Its value is affected by slow leakage in between tests.

\section{Test 960826}

Data for this test are illustrated in Figures 1,3 and 4 (attachment 3 ), and results are collected in Tables 2 and 4 . This al iquot al so generated condensate and noncondensible gas upon heatup. Comparing the sums from the two calculation methods for the first two tests with the corresponding value in test 960826 suggests that the auxiliary data method gives more internally consistent results in this instance.

Table 2. U-106 Auxiliary Test Data.

\begin{tabular}{|c|c|c|c|c|c|c|c|c|c|c|}
\hline \multirow[b]{2}{*}{ Test 10} & \multicolumn{3}{|c|}{$\begin{array}{l}\text { At start of run } \\
\text { sample at ambient } T\end{array}$} & \multicolumn{2}{|c|}{$\begin{array}{l}\text { At Peak of Run } \\
\text { Sample ot } T\end{array}$} & \multicolumn{4}{|c|}{$\begin{array}{l}\text { At and of run } \\
\text { cample cooled to amblent } T\end{array}$} & \multirow[b]{2}{*}{ Comment } \\
\hline & $\begin{array}{c}P_{0} \\
\text { (barg) }\end{array}$ & $\begin{array}{c}\mathrm{T}_{0} \\
\left(1^{\circ} \mathrm{C}\right)\end{array}$ & $w_{s}^{\text {prep }}$ & $\begin{array}{c}\left({ }^{\circ} \mathrm{C}\right) \\
\text { Observed } \\
\text { at } t \text { (min) } \\
\text { from } \\
\text { Onset }\end{array}$ & $\begin{array}{l}\text { max. P (barg) } \\
\text { Observed at } t \\
\text { (min) from } \\
\text { Onset }\end{array}$ & $\underset{\text { (barg) }}{\mathbf{P}_{f}}$ & $\begin{array}{c}T_{f} \\
\left({ }^{\circ} \mathrm{C}\right)\end{array}$ & $\begin{array}{c}w_{8}^{\text {react }} \\
\text { (0) }\end{array}$ & $\%\left(\Delta w_{8} / w_{s}\right)^{\text {react }}$ & \\
\hline 960815 & 6.9843 & 20.84 & 10.67 & $\begin{array}{c}212^{\circ} \mathrm{C} \\
\text { no onset }\end{array}$ & $\begin{array}{c}8.537 \text { barg } \\
\text { no onset }\end{array}$ & 7.2167 & 21.62 & & & $\begin{array}{c}\text { Hestup } \\
\text { completed next } \\
\text { test }\end{array}$ \\
\hline 960819 & 7.2167 & 21.62 & 10.57 & $\begin{array}{c}340^{\circ} \mathrm{C} \text { at } \\
64 \mathrm{~min}\end{array}$ & $\begin{array}{l}9.86 \text { barg } \\
\text { at } 101 \text { min } \\
\text { trom onsot }\end{array}$ & 7.6934 & 25,08 & 9.64 & .8 .80 & Reheat of above \\
\hline 960826 & 6.9873 & 25.52 & 9.24 & $\begin{array}{l}400^{\circ} \mathrm{C} \mathrm{at} \\
81.2 \mathrm{~min}\end{array}$ & $\begin{array}{l}9.5 \text { barg at } \\
103.5 \text { min } \\
\text { from onset }\end{array}$ & 7.3562 & 22.2 & 8.25 & -10.71 & \\
\hline
\end{tabular}


F. H. Steen

Page 6

September 9, 1996.

Table 3. U-106 Self-Heat Results.

\begin{tabular}{|c|c|c|c|c|c|c|}
\hline Test ID & $\begin{array}{l}\mathrm{T}_{\text {onat }} \\
\left({ }^{\circ} \mathrm{C}\right)\end{array}$ & $\begin{array}{l}\Delta T_{\text {true }} \\
\left(^{\circ} \mathrm{C}\right)\end{array}$ & $\begin{array}{l}\text { Max. dT/dt } \\
\text { (uncorr.) } \\
\left({ }^{\circ} \mathrm{C} / \mathrm{min}\right) \text {, } \\
\text { at T }\left({ }^{\circ} \mathrm{C}\right)^{\circ} \\
\text { or } \mathrm{t}(\mathrm{min}) \\
\text { from onset }\end{array}$ & $\begin{array}{l}\text { Initial E, } \\
\text { (KJ/mole) }\end{array}$ & $\begin{array}{c}\text { Initial } \mathrm{K}\left(\mathrm{C}^{\circ} / \mathrm{min}\right) \text { at } \\
\mathrm{T}\left({ }^{\circ} \mathrm{C}\right)\end{array}$ & Comments \\
\hline 960815 & none & none & none & none & none & $\begin{array}{c}\text { Test } \\
\text { canceled } \\
\text { before self- } \\
\text { heat }\end{array}$ \\
\hline 960810 & 225 & $\phi \times 63.2$ & $\begin{array}{c}\phi \times 42.1^{\circ} \mathrm{C} / \mathrm{min} \\
\text { at } 319^{\circ} \mathrm{C} \\
\text { or } 54.9 \mathrm{~min} \\
\text { from onset }\end{array}$ & $146 \pm 21$ & $\begin{array}{c}\phi \times(0.74 \pm 0.24) \\
\text { at } 250^{\circ} \mathrm{C}\end{array}$ & $\begin{array}{l}\text { B fixed at } \\
0.81^{\circ} \mathrm{C} / \mathrm{min}\end{array}$ \\
\hline 960826 & 210 & $\phi \times 119$ & $\begin{array}{c}\times 14.0^{\circ} \mathrm{C} / \mathrm{min} \\
\text { at } 373^{\circ} \mathrm{C} \\
\text { or } 75.9 \mathrm{~min} \\
\text { from onset }\end{array}$ & $160 \pm 11$ & $\begin{array}{c}\phi \times(1.39 \pm 0.12) \\
\text { at } 300^{\circ} \mathrm{C}\end{array}$ & $\begin{array}{l}\text { B fixed at } \\
0.87^{\circ} \mathrm{C} / \mathrm{min}\end{array}$ \\
\hline
\end{tabular}

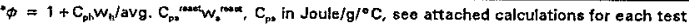

Table 4. U-106 Gas Production Results.

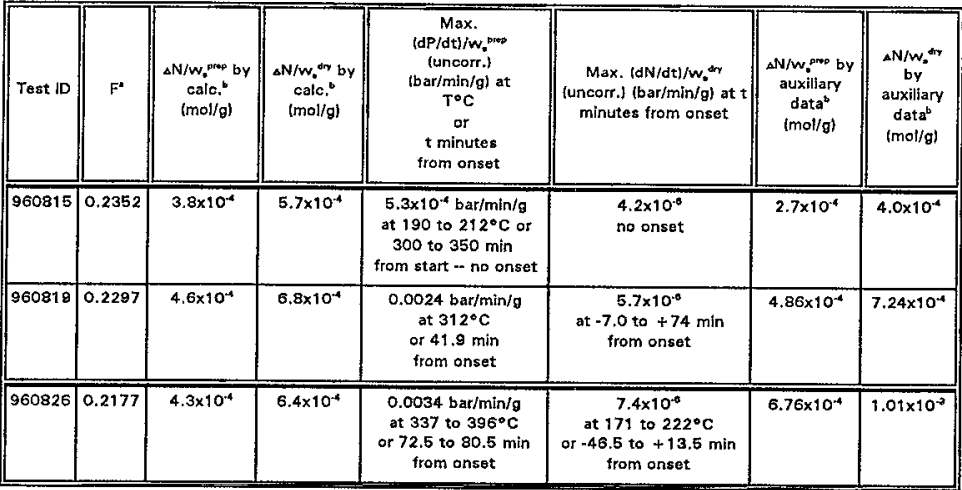

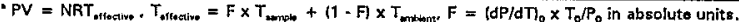

Noncondensible gases only 
F. H. Steen

Page 7

75764-PCS96-091

September 9, 1996 .

Feel free to call if you have any further questions on this matter.

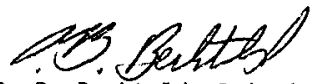

D. B. Bechtold, Principal Scientist

Process Chemistry \& Statistics

dls

Attachments (3) 


\author{
75764-PCS96-091 \\ Attachment 1
}

Figures and Calculations for RSST Results of Test 960815;

Sample U-106, S96T004013

Consisting of 7 Pages including this cover page 
WHC-SD-WM-DP-191, REV. |

Test 960815; Sample U-106, S96T004013

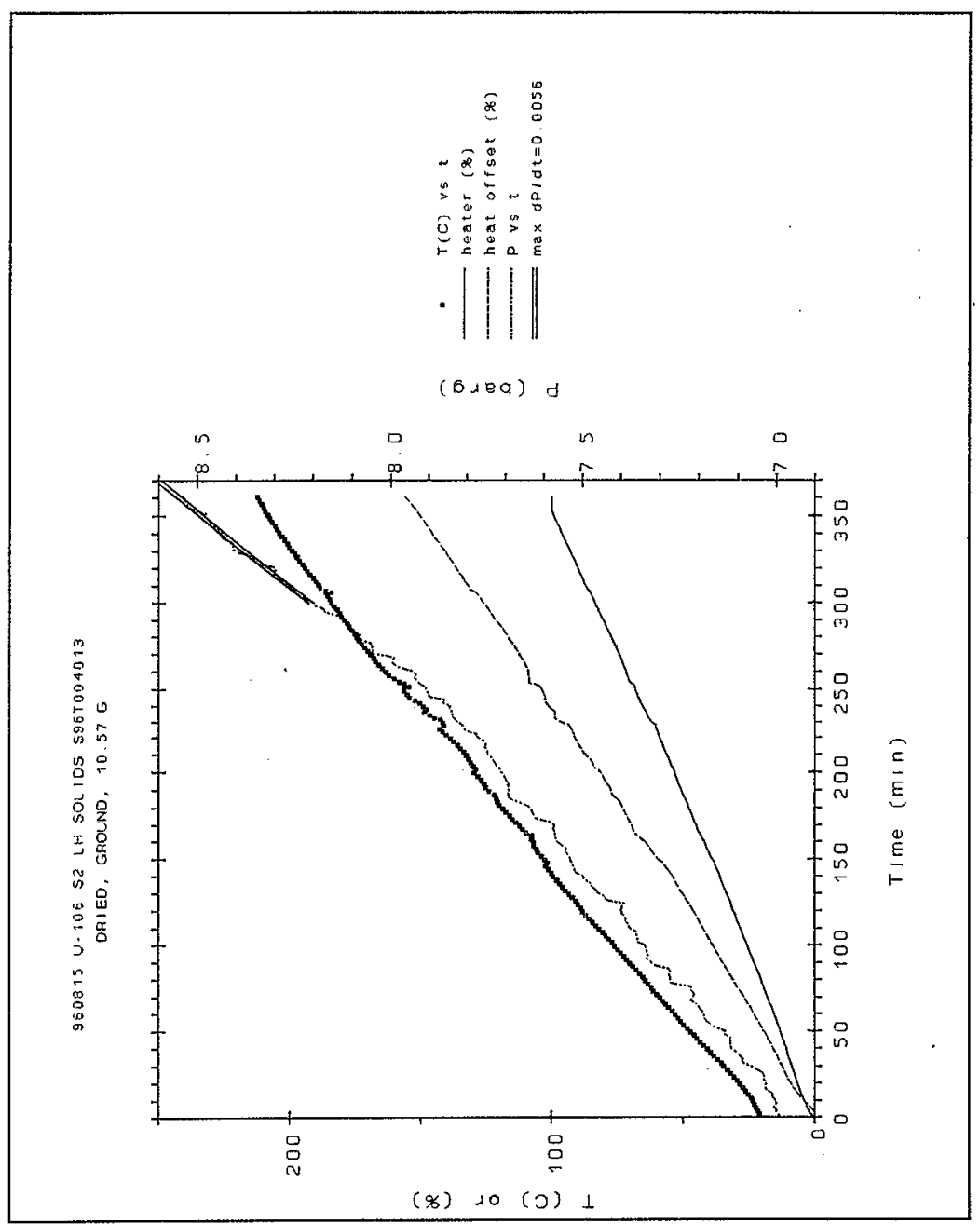

Figure I 
WHC-SD-WM-DP-191, REV. I

Test 960815; Sample U-106, S96T004013

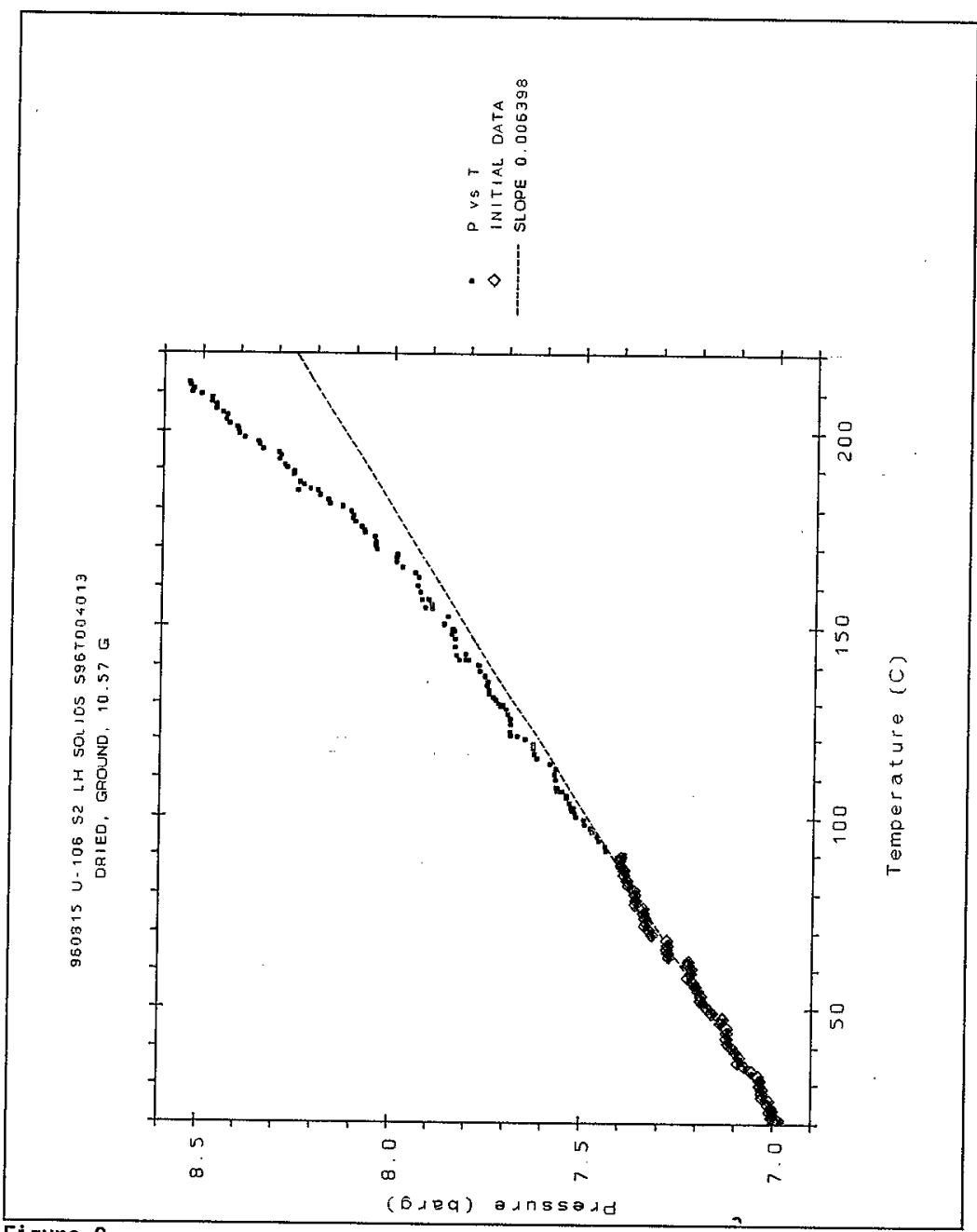

Figure 2 


\section{WHC-SD-WM-DP-191, REV.I}

Test 960815; Sample U-106, S96T004013

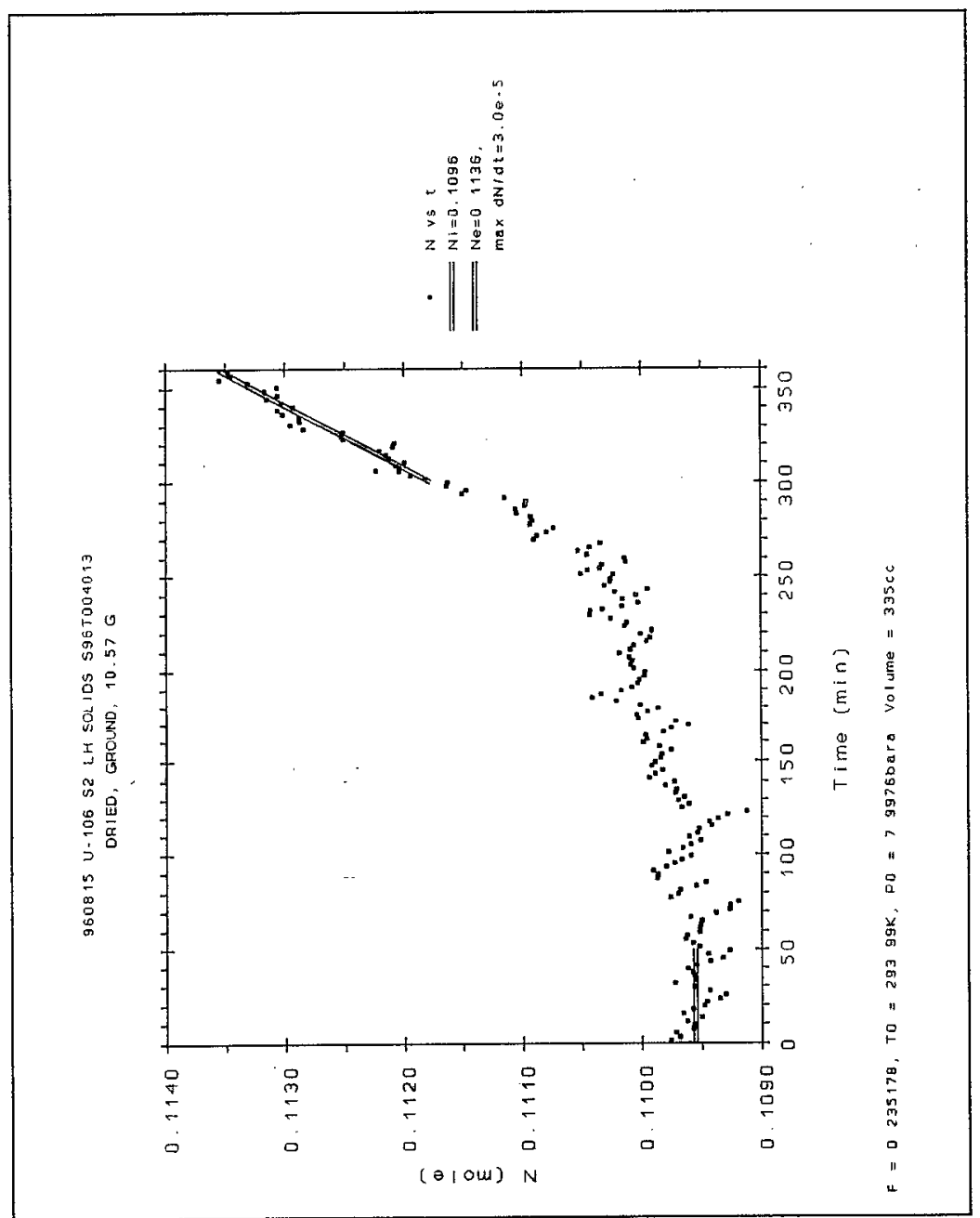

Figure 3 


\section{WHC-SD-WM-DP-191, REV. I}

Test 960815; Sample U-106, S96T004013

WEIGHT LOSSES

$$
\begin{aligned}
& { }_{8} \mathrm{H}_{2} \mathrm{O} \text { prep }=100 \frac{\text { gross }^{\text {wet }}-\text { gross }^{\text {prep }}}{\text { gross wet }- \text { tare prep }} \\
& =100 \frac{214.4-209.7}{214.4-187.5}=17.478 \\
& 8 \mathrm{H}_{2} \mathrm{O} \text { dry }=44.57 \% \text { (determined by separate analyses) } \\
& \%\left(\frac{\Delta w_{s}}{w_{s}}\right)^{\text {react }}=100 \frac{\text { gross }}{\text { react }- \text { gross }_{s}} \\
& \text { = (See Calculations Test 960819) } \\
& =100-\frac{1}{100}\left[100-8 \mathrm{H}_{2} \mathrm{O}^{\text {prep }}\right]\left[100+8\left(\frac{\Delta \mathrm{w}_{\mathrm{s}}}{\mathrm{w}_{\mathrm{s}}}\right)^{\text {react }}\right] \\
& =\text { (See Calculations Test 960819) }
\end{aligned}
$$

\% (total volatiles $)^{\text {react }}=100-\%$ solids ${ }^{\text {react }}$ 
TOTAL SPECIFIC NONCONDENSIBLE GAS PRODUCTION:

$$
\begin{aligned}
& \frac{\left(\frac{d P}{d t}\right)_{\text {uncoxz }}^{\max }}{w_{s}^{\text {pxep }}}=\frac{0.0056}{10.57}=5.3 \times 10^{-4} \frac{\text { bax }}{\min g^{\text {prep }}} \\
& \text { at } 300 \text { to } 350^{\circ} \mathrm{C}
\end{aligned}
$$

By CaIcylated Graph:

$$
\begin{aligned}
& \mathrm{N}_{\mathrm{i}}=0.1096 \text { mole, } \mathrm{N}_{\theta}=0.1136 \text { mole, } \mathrm{w}_{\mathrm{s}}^{\text {prep }}=10.57 \mathrm{grams} \\
& \frac{\Delta N}{W_{s}^{\text {prep }}}=\frac{0.1136-0.1096}{10.57}=3.8 \times 10^{-4} \frac{\text { mole }}{\text { gram prep }} \\
& \frac{\Delta N}{W_{S}^{d r y}}=\frac{\Delta N}{W_{s}^{\text {prep }}} \frac{100-8 \mathrm{H}_{2} \circ \text { prep }}{100-8 \mathrm{H}_{2} \mathrm{Odry}}=3.8 \times 10^{-4} \frac{100-17.47}{100-44.57} \\
& =5.7 \times 10^{-4} \frac{\text { mole }}{\text { gram dxy }} \\
& \frac{\left(\frac{d N}{d t}\right)_{\text {uncoxI }}^{\max }}{w_{s}^{\text {prep }}}=\frac{3.0 \times 10^{-5}}{10} \cdot 57=2.8 \times 10^{-6} \frac{\text { mole }}{\text { gram prep }} \\
& \frac{\left(\frac{d N}{d t}\right)_{\text {uncorr }}^{\max }}{w_{s}^{d x y}}=2.8 \times 10^{-6} \frac{100-17.47}{100-44.57}=4.2 \times 10^{-6} \frac{\text { mole }}{\text { gram dry }} \\
& \text { at } 300 \text { to } 360^{\circ} \mathrm{C}
\end{aligned}
$$




\section{WHC-5D-WM-DP-191, REV, I}

Test 960815; Sample U-106, S96T004013

By Auxiliary Data:

$$
\begin{aligned}
& P_{0}=6.9843 \text { barg, } T_{0}=20.84^{\circ} \mathrm{C}, \quad P_{E}=7.2167 \text { barg, } T_{f}=21.62^{\circ} \mathrm{C} \\
& V=335 \mathrm{~cm}^{3}, \quad R=83.14 \frac{\text { bara }^{3}}{\operatorname{mole} 0^{\circ} \mathrm{K}} \\
& \frac{\Delta N}{w_{s}^{\text {pxep }}}=\frac{1}{W_{s}^{\text {prep }}} \frac{V}{R}\left[\frac{P_{f}+1.01325}{T_{f}+273.15}-\frac{P_{0}+1.01325}{T_{0}+273.15}\right] \\
& =\frac{1}{10.57} \frac{335}{83.14}\left[\frac{7.2167+1.01325}{21.62+273.15}-\frac{6.9843+1.01325}{20.84+273.15}\right] \\
& =2.7 \times 10^{-4} \frac{\text { mole }}{\text { gram prep }} \\
& \frac{\Delta N}{w_{s}^{d x y}}=2.7 \times 10^{-4} \frac{100-17.47}{100-44.57}=4.0 \times 10^{-4} \frac{\text { mole }}{\text { gram dry }}
\end{aligned}
$$




\section{WHC-SD-WM-DP-191, REV. |}

\section{4-PCS96-091}

Attachment 2

Figures and Calculations for RSST Results of Test 950819;

Sample U-106, S96T004013

Consisting of 11 Pages including this cover page 


\section{WHC-SD-WM-DP-191, REV. 1}

Test 960819; Sample U-106, S96T004013

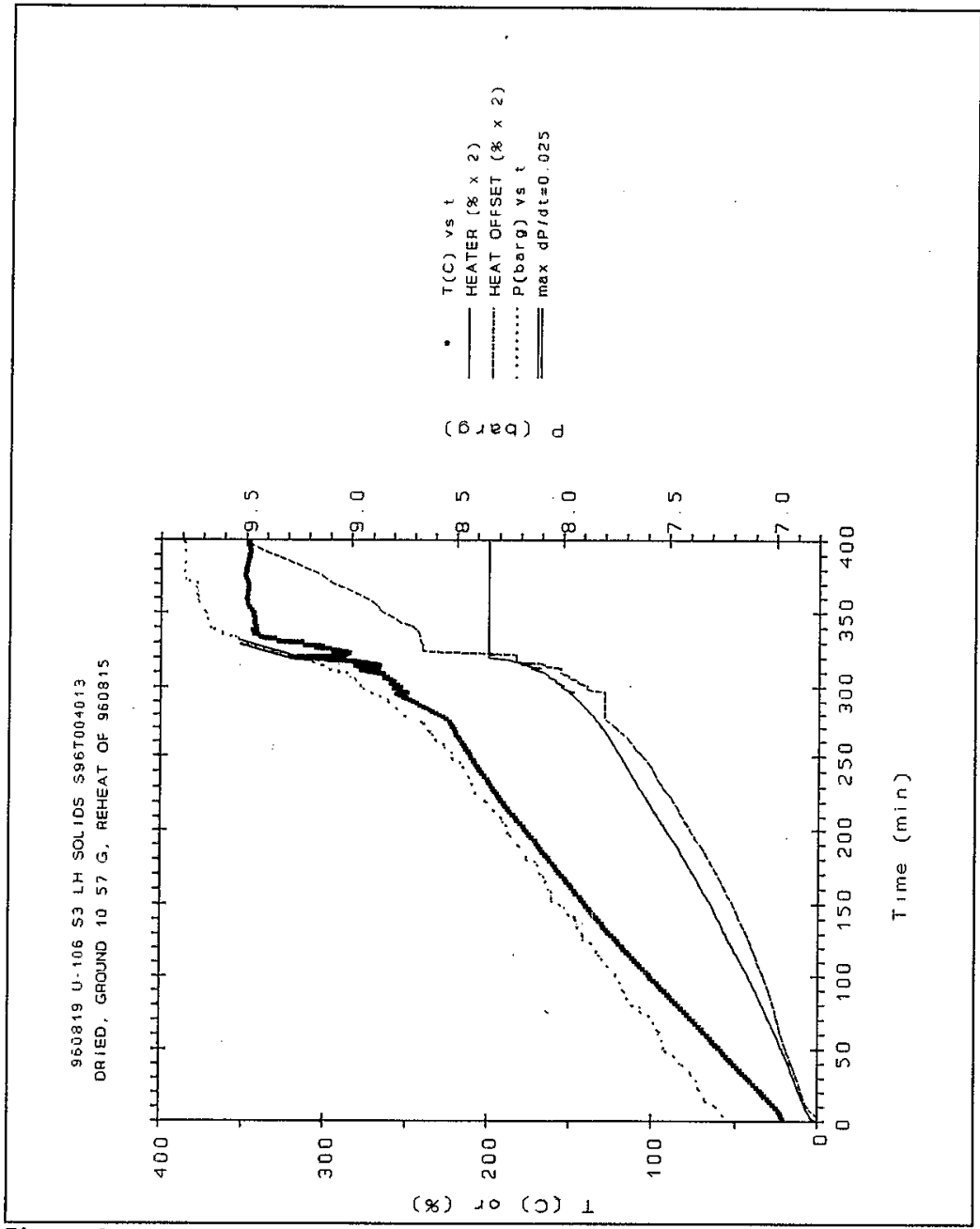

Figure 1 
WHC-SD-WM-DP-191, REV.|

Test 960819; Sample U-106, S96T004013

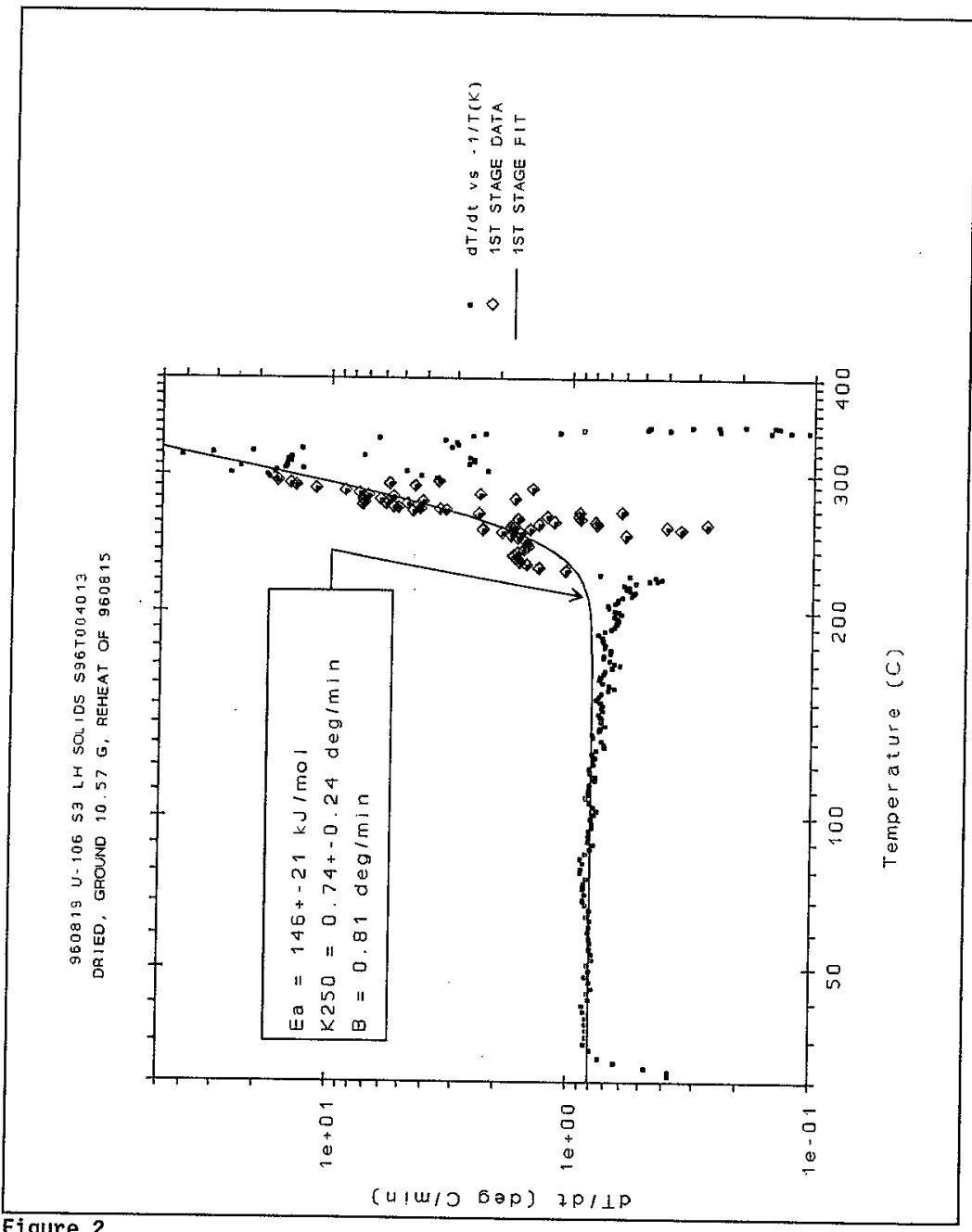

Figure 2 
WHC-SD-WM-DP-191, REV. $\mid$

Test 960819; Sample U-106, S96T004013

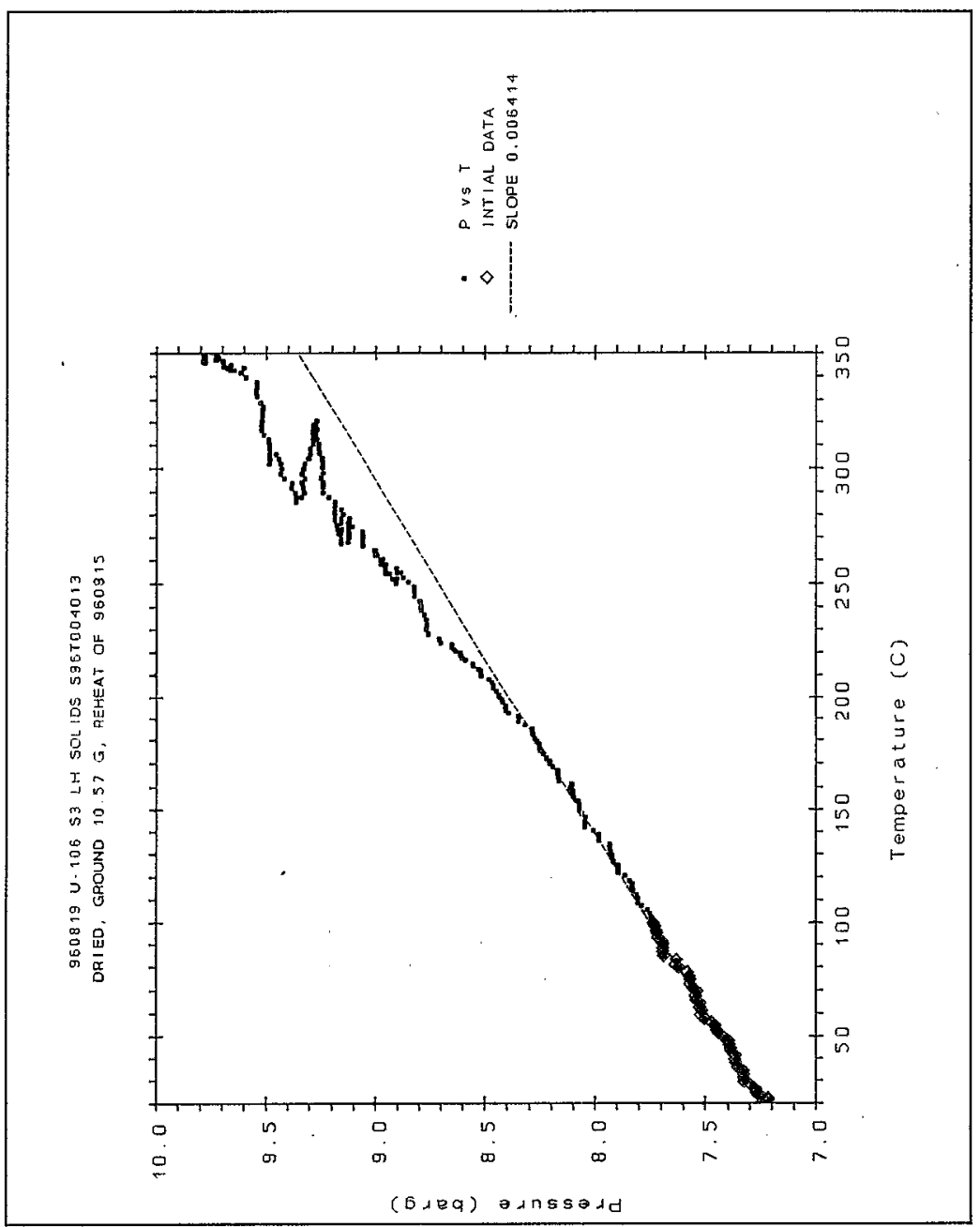

Figure 3 
WHC-SD-WM-DP-191, REV.|

Test 960819; Sample U-106, S96T004013

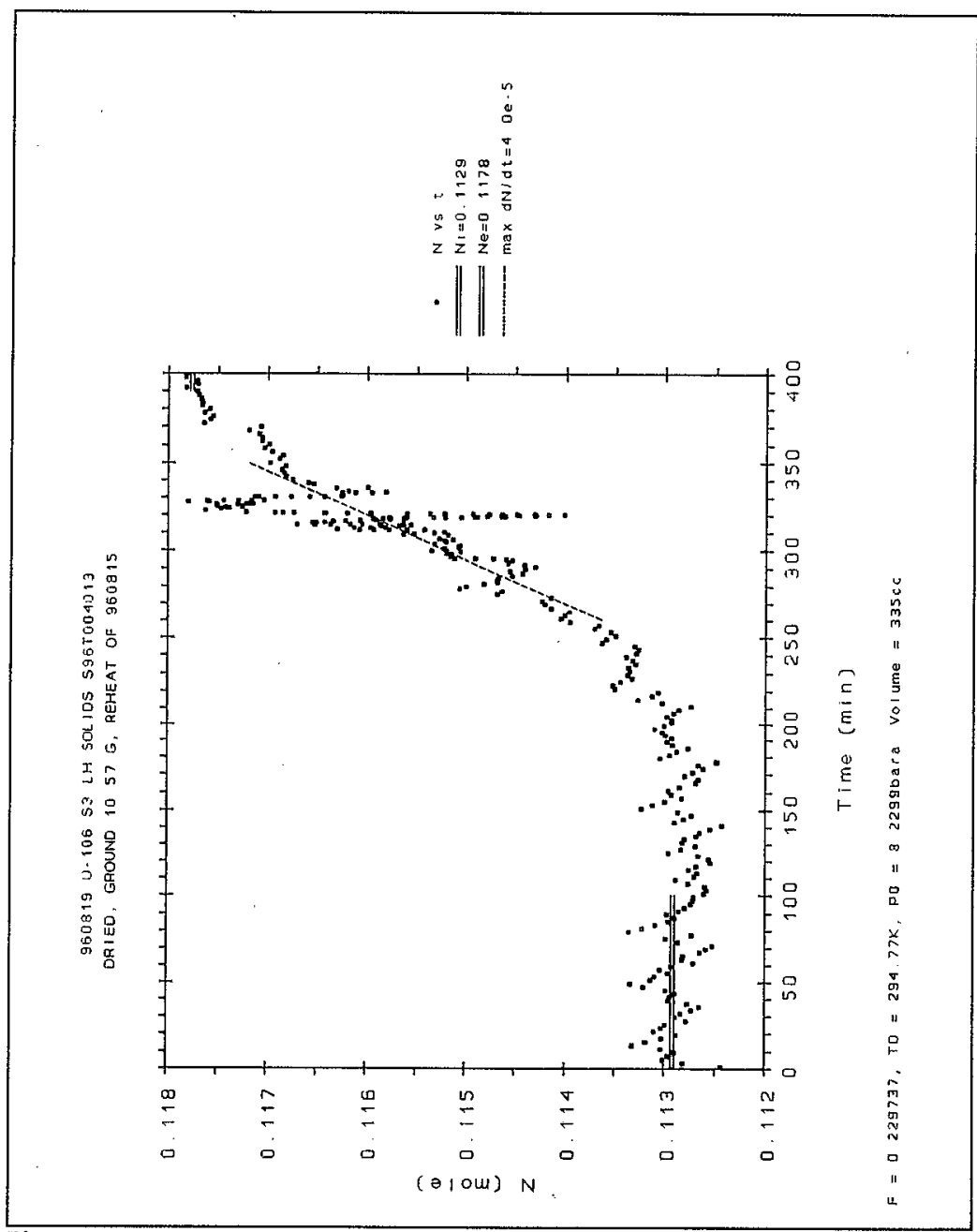

Figure 4 


\section{WHC-SD-WM-DP-191, REV.I}

Test 960819; Sample U-106, S96T004013

WEIGHT LOSSES

$$
\begin{aligned}
& 8 \mathrm{H}_{2} \mathrm{O} \text { prep }=100 \frac{\text { gross }}{\text { wet }- \text { gross prep }} \\
& \text { = See Calculations Test } 960815 \\
& 8_{8} \mathrm{H}_{2} \mathrm{O} d r y=\text { See Calculations Test } 960815 \\
& 8\left(\frac{\Delta W_{s}}{W_{s}}\right)^{\text {react }}=100 \frac{\text { gross }_{\text {react }}-\text { gross }_{s}}{\text { gross }_{s}-\text { tare }_{s}} \\
& =100 \frac{59.84-60.77}{60.77-50.20}=-8.80 \%
\end{aligned}
$$

$\%(\text { total volatiles })^{\text {react }}=100-\%$ solids ${ }^{\text {react }}$

$$
\begin{aligned}
& =100-\frac{1}{100}\left[100-8 \mathrm{H}_{2} \mathrm{O} \text { prep }\right]\left[100+8\left(\frac{\Delta w_{s}}{w_{s}}\right)^{\text {react }}\right] \\
& =100-\frac{1}{100}[100-17.47][100-8.80] \\
& =24.73 \%
\end{aligned}
$$




\section{WHC-SD-WM-DP-191, REV.}

Test 960819; Sample U-106, S96T004013

TOTAL SPECIFIC NONCONDENSIBLE GAS PRODUCTION:

$$
\begin{aligned}
\frac{\left(\frac{d P}{d t}\right)_{\text {uncorx }}^{\max }}{w_{\mathrm{s}}^{\text {prep }}}= & \frac{0.025}{10.57}=0.0024 \frac{\text { bax }}{\min g^{\text {prep }}} \text { at } 312^{\circ} \mathrm{C} \\
& \text { or } 325.0-276.0=49.0 \mathrm{~min} . \text { from onset }
\end{aligned}
$$

By Calculated Graph:

$$
\begin{aligned}
N_{i} & =0.1129 \text { mole, } N_{\theta}=0.1178 \text { mole, } w_{s}^{\text {prep }}=10.57 \text { grams } \\
\frac{\Delta N}{W_{s}^{\text {prep }}} & =\frac{0.1178-0.1129}{10.57}=4.6 \times 10^{-4} \frac{\text { mole }}{\text { gram prep }} \\
\frac{\Delta N}{W_{s}^{d r y}} & =\frac{\Delta N}{w_{\mathrm{g}}^{\text {prep }}} \frac{100-8 \mathrm{H}_{2} \mathrm{O} \text { prep }}{100-8 \mathrm{H}_{2} \mathrm{O}^{\mathrm{dry}}}=4.6 \times 10^{-4} \frac{100-17.47}{100-44.57} \\
& =6.8 \times 10^{-4} \frac{\text { mole }}{\text { grard dry }}
\end{aligned}
$$

$$
\begin{aligned}
& \frac{\left(\frac{d N}{d t}\right)_{\text {uncorx }}^{\max }}{w_{s}^{\text {prep }}}=\frac{4.0 \times 10^{-5}}{10} \cdot 57=3.8 \times 10^{-6} \frac{\text { mole }}{\text { gram prep }} \\
& \frac{\left(\frac{d N}{d t}\right)_{\text {uncorr }}^{\max }}{w_{s}^{d x y}}=3.8 \times 10^{-6} \frac{100-17.47}{100-44.57}=5.7 \times 10^{-6} \frac{\text { mole }}{\text { gram dry }}
\end{aligned}
$$$$
\text { at } 218 \text { to } 344^{\circ} \mathrm{C} \text { or }-7.0 \text { to }+74 \mathrm{~min} \text { from onset }
$$ 


\section{WHC-SD-WM-DP-191, REV.I}

Test 960819; Sample U-106, S96T004013

By Auxiljary Data:

$\mathrm{P}_{0}=7.2167$ barg, $\mathrm{T}_{0}=21.62^{\circ} \mathrm{C}, \mathrm{P}_{\mathrm{f}}=7.6934$ barg, $\mathrm{T}_{\mathrm{f}}=25.08{ }^{\circ} \mathrm{C}$

$$
\mathrm{V}=335 \mathrm{~cm}^{3}, \quad \mathrm{R}=83.14 \frac{\mathrm{bara} \mathrm{cm}^{3}}{\operatorname{mole}^{\circ} \mathrm{K}}
$$

$$
\begin{aligned}
\frac{\Delta N}{W_{s}^{\text {prep }}} & =\frac{1}{W_{s}^{\text {prep }}} \frac{V}{R}\left[\frac{P_{f}+1.01325}{T_{E}+273.15}-\frac{P_{0}+1.01325}{T_{0}+273.15}\right] \\
& =\frac{1}{10.57} \frac{335}{83.14}\left[\frac{7.6934+1.01325}{25.08+273.15}-\frac{7.2167+1.01325}{21.62+273.15}\right] \\
& =4.86 \times 10^{-4} \frac{\text { mole }}{\text { gram prep }} \\
\frac{\Delta N}{W_{s}^{\text {dry }}} & =4.86 \times 10^{-4} \frac{100-17.47}{100-44.57}=7.24 \times 10^{-4} \frac{\text { mole }}{\text { gram dry }}
\end{aligned}
$$




\section{WHC-SD-WM-DP-191, REV.I}

Test 960819; Sample U-106, S96T004013

\section{SELF-HEAT PARAMETERS}

$\Delta \mathrm{T}_{\text {true }}$ :

$$
\begin{aligned}
& T_{i} \approx 225^{\circ} \mathrm{C}, t_{i} \approx 276.0 \mathrm{~min}, \mathrm{~T}_{\theta} \approx 340^{\circ} \mathrm{C}, t_{\theta} \approx 340.0 \mathrm{~min} \\
& \mathrm{~B} \approx 0.81^{\circ} \mathrm{C} / \mathrm{min}, \\
& \left.\Delta T_{\text {true }}=\phi \Delta T_{\text {corx }}=\phi\left(T_{e}-T_{1}\right]-B\left[t_{\theta}-t_{1}\right]\right) \\
& =\phi([340-225]-0.81[340.0-276.0]) \\
& =\phi 63.2^{\circ} \mathrm{C} \\
& \phi=\frac{\left(\bar{C}_{p s}^{\text {react }}\right)\left(w_{s}^{\text {react }}\right)+C_{p h} w_{h}}{\left(\bar{C}_{p s}^{\text {react }}\right)\left(w_{s}^{\text {react }}\right)}=\left(1+\frac{C_{p h} w_{h}}{\left(\bar{C}_{p s}^{\text {react }}\right) \frac{w_{s}^{\text {react }}}{w_{s}^{\text {ped }}} w_{s}^{\text {prep }}}\right) \\
& =1+\left(\frac{C_{p h} w_{h}}{\left(\bar{C}_{p s}^{\text {react }}\right) \frac{100+\left(8 \Delta \frac{w_{s}}{w_{s}}\right)^{\text {react }}}{100} w_{s}^{\text {prep }}}\right) \\
& =1+\left(\frac{0.8368}{\left(\overline{\mathrm{C}}_{\mathrm{ps}}^{\text {react }}\right) \frac{100+(-8.80)}{100} 10.57}\right) \\
& =1+\frac{0.087}{\left(\bar{C}_{p s}^{\text {react }}\right)} \quad\left(\left(\bar{C}_{p s}^{\text {react }}\right) \text { units } \frac{J}{g^{\top} K}\right) \\
& \left(\frac{d T}{d t}\right)_{\text {uncorx }}^{\max }=42.1 \frac{\cdot \mathrm{c}}{\min } \text { at } 319^{\circ} \mathrm{C} \\
& \text { or } 330.9-276.0=54.9 \mathrm{~min} \text { from onset }
\end{aligned}
$$




\section{WHC-SD-WM-DP-191, REV.}

\section{Test 960819; Sample U-106, S96T004013}

\section{THERMAL KINETIC PARAMETERS:}

For the initial stage of the runaway, $d T / d t$ vs $Z=-1000 /(T+273.15)$ data for temperatures between $225^{\circ} \mathrm{C}$ and $295^{\circ} \mathrm{C}$ and for $\mathrm{dT} / \mathrm{dt}$ values greater than $0.1^{\circ} \mathrm{C} / \mathrm{min}$ are least-squares fit by the function

$$
\begin{gathered}
\frac{d T}{d t}=B+K_{250} e^{\frac{E_{n}}{R}\left(z-z_{250}\right)}, \\
\text { where } z=-\frac{1000}{T+273.15}, Z_{250}=-\frac{1000}{250+273.15}
\end{gathered}
$$

with parameters $E_{a}=146 \pm 21 \mathrm{KJ} /$ mole, $K_{250}=0.74 \pm 0.23{ }^{\circ} \mathrm{C} / \mathrm{min}$; and constants $B=0.81{ }^{\circ} \mathrm{C} / \mathrm{min}$ and $R=8.3144^{25} \mathrm{~J} / \mathrm{mole} /{ }^{\circ} \mathrm{K}$. 


\section{WHC-SD-WM-DP-191, REV.I}

Test 960819; Sample U-106, S96T004013

\section{SPECIFIC EXOTHERMIC ENERGY RELEASE:}

Neglecting PV work,

$$
\begin{aligned}
& Q \approx \Delta T_{\text {true }}\left(w_{s}^{\text {react }}\right)\left(\overline{\mathrm{C}}_{\mathrm{ps}}^{\text {react }}\right)=\Delta \mathrm{T}_{\text {corr }} \Phi\left(\mathrm{W}_{\mathrm{s}}^{\text {react }}\right)\left(\overline{\mathrm{C}}_{\mathrm{ps}}^{\text {react }}\right) \\
& =\Delta T_{\text {corx }} \frac{\left[\left(w_{s}^{\text {react }}\right)\left(\overline{\mathrm{C}}_{\mathrm{ps}}^{\text {react }}\right)+\mathrm{w}_{\mathrm{h}} \mathrm{C}_{\mathrm{ph}}\right]}{\left(\mathrm{w}_{\mathrm{s}}^{\text {react }}\right)\left(\overline{\mathrm{C}}_{\mathrm{ps}}^{\text {react }}\right)}\left(\mathrm{w}_{\mathrm{s}}^{\text {react }}\right)\left(\overline{\mathrm{C}}_{\mathrm{ps}}^{\text {react }}\right) \\
& =\Delta \mathrm{T}_{\text {cors }}\left[\left(\mathrm{w}_{\mathrm{s}}^{\text {react }}\right)\left(\overline{\mathrm{C}}_{\mathrm{ps}}^{\text {react }}\right)+\mathrm{w}_{\mathrm{h}} \mathrm{C}_{\mathrm{ph}}\right] \\
& \hat{Q}_{d x y} \approx \Delta T_{\operatorname{corI}}\left[\frac{w_{s}^{\text {react }}}{w_{s}^{\text {dry }}}\left(\bar{C}_{p s}^{\text {react }}\right)+\frac{w_{h} C_{p h}}{w_{s}^{d x y}}\right]
\end{aligned}
$$

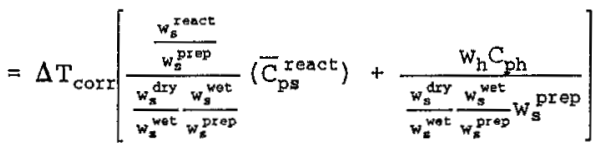

$$
\begin{aligned}
& =\Delta T_{\operatorname{corx}} \frac{\frac{w_{s}^{\text {pros }}}{w_{s}^{\text {wet }}}}{\frac{w_{s}^{\text {dry }}}{w_{s}^{\text {wet }}}}\left[\frac{w_{s}^{\text {yeact }}}{w_{s}^{\text {prep }}}\left(\vec{C}_{p s}^{\text {react }}\right)+\frac{w_{h} C_{p h}}{w_{s}^{\text {pxep }}}\right] \\
& \hat{Q}_{\mathrm{dry}} \approx \Delta \mathrm{T}_{\mathrm{corx}} \frac{\left(100-8 \mathrm{H}_{2} \mathrm{O}^{\text {prep }}\right)}{\left(100-8 \mathrm{H}_{2} \mathrm{O}^{\mathrm{dry}}\right)}\left[\frac{100+\left(8 \frac{\Delta \mathrm{w}_{s}}{\mathrm{w}_{s}}\right)^{\text {react }}}{100}\left(\overline{\mathrm{C}}_{\mathrm{ps}}^{\text {react }}\right)+\frac{\mathrm{w}_{\mathrm{h}} \mathrm{C}_{\mathrm{ph}}}{\mathrm{w}_{\mathrm{s}}^{\mathrm{prep}}}\right] \\
& =63.2 \frac{(100-17.47)}{(100-44.67)}\left[\frac{100+(-8.80)}{100}\left(\overline{\mathrm{C}}_{\mathrm{ps}}^{\text {react }}\right)+\frac{0.8368}{10.57}\right] \\
& =85.8\left(\overline{\mathrm{C}}_{\text {ps }}^{\text {react }}\right)+7.45 \frac{\mathrm{J}}{\mathrm{g}^{\text {sxy }}}
\end{aligned}
$$


WHC-SD-WM-DP-191, REV. I

\section{4-PCS96-091}

Attachment 3

Figures and CaTculations for RSST Results of Test 960826;

Sample U-106, S96T004013

Consisting of 11 Pages including this cover page 


\section{WHC-SD-WM-DP-191, REV. |}

Test 960826; Sample U-106, S96T004013

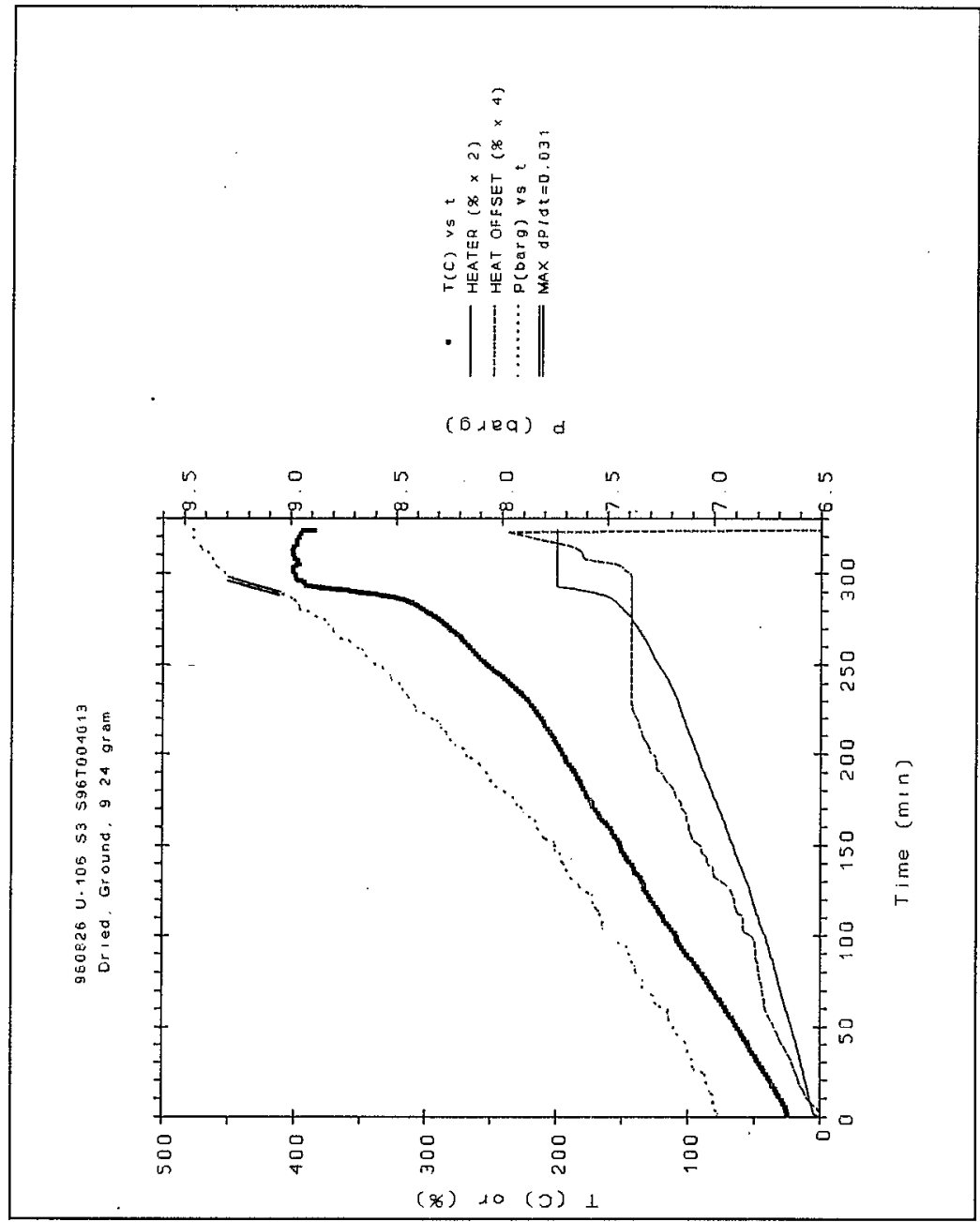

Figure 1 
WHC-SD-WM-DP-191, REV.I

Test 960826; Sample U-106, S96T004013

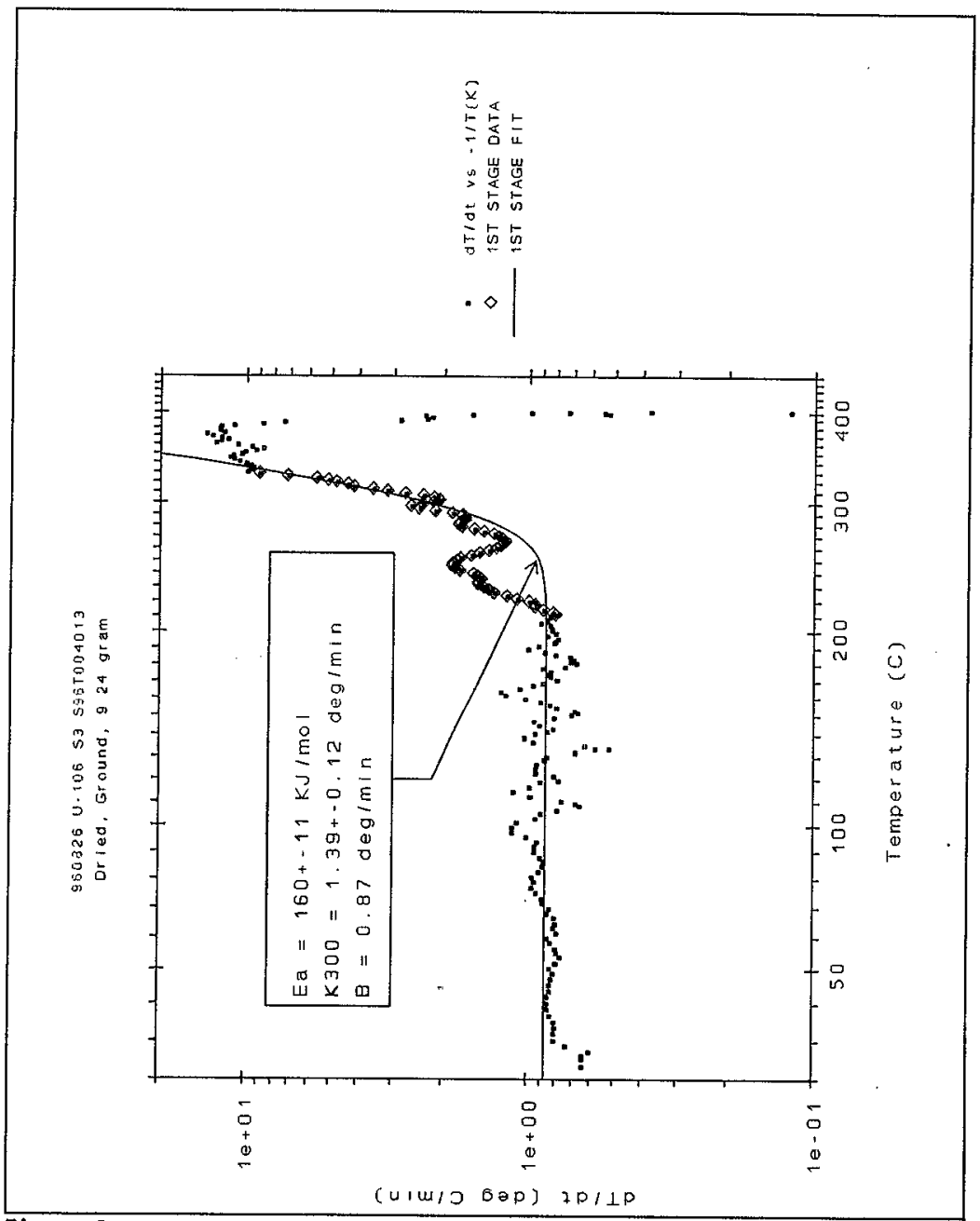

Figure 2 
WHC-SD-WM-DP-191, REV.I

Test 960826; Sample U-106, S96T004013

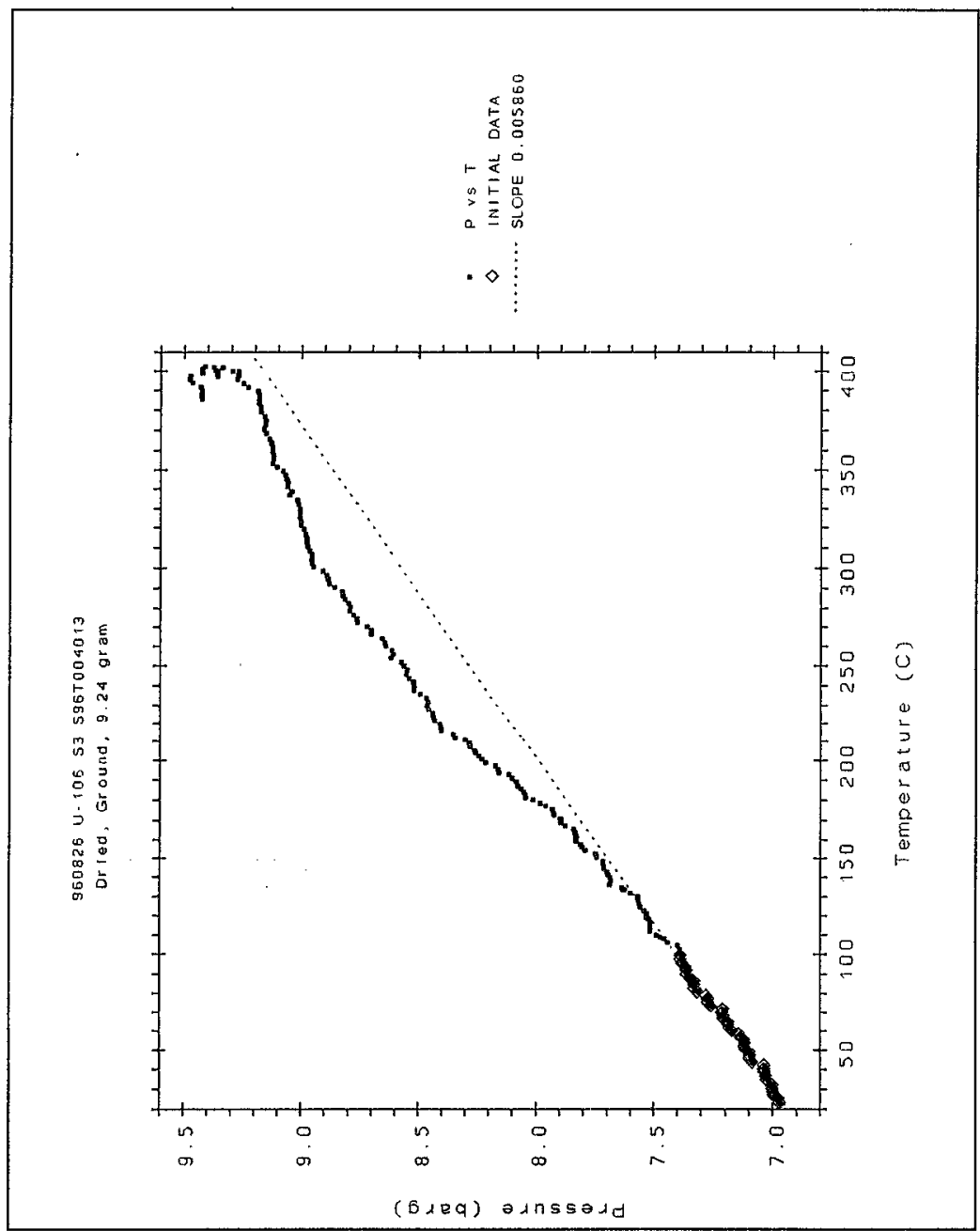

Figure 3 
WHC-SD-WM-DP-191, REV. I

Test 960826; Sample U-106, S96T004013

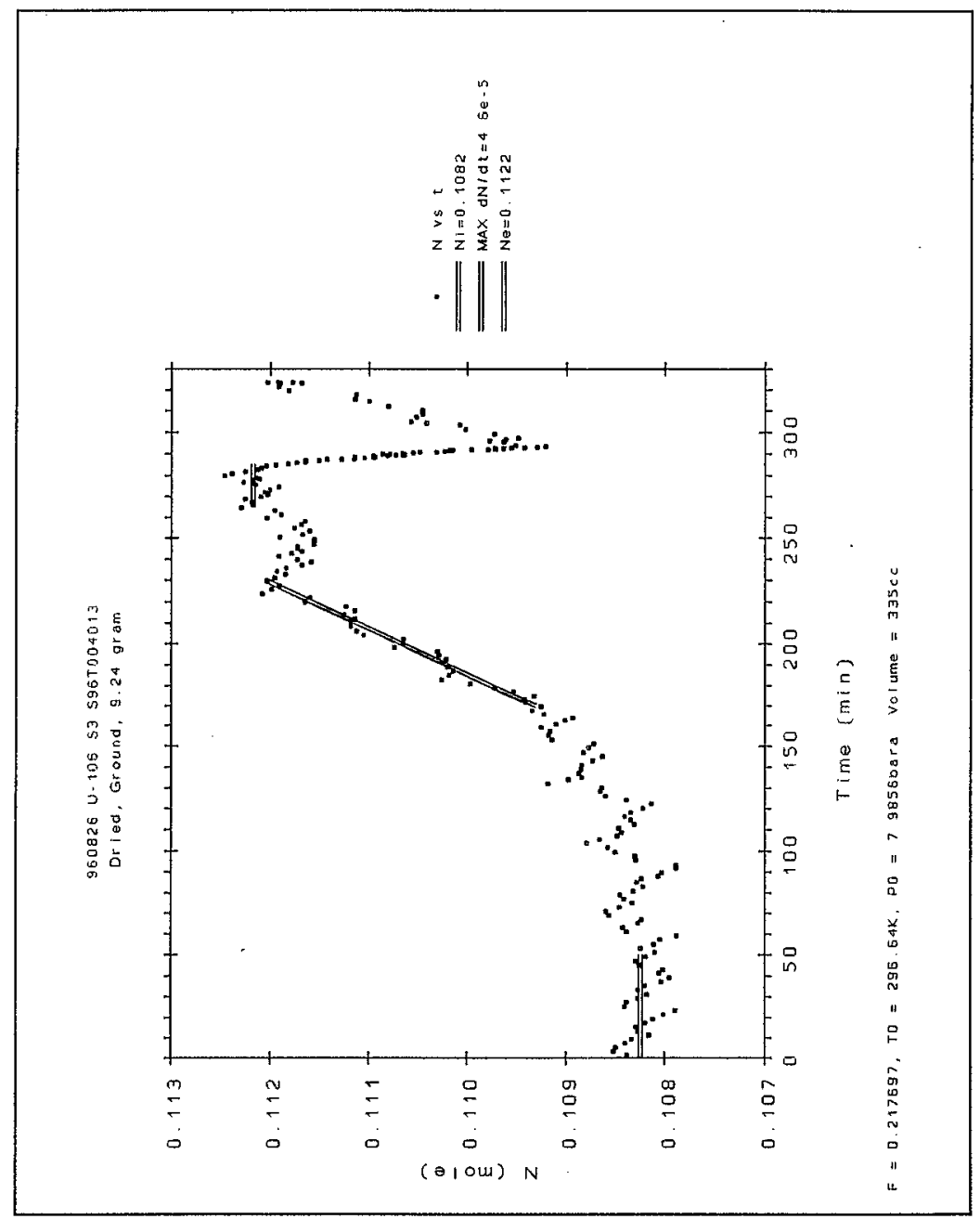

Figure 4 
Test 960826; Sample U-106, S96T004013

WEIGHT LOSSES

$$
\begin{aligned}
8_{2} \mathrm{H}_{2} \mathrm{O}^{\text {prep }} & =100 \frac{\text { gross wet }- \text { gross prep }}{\text { gross wet }- \text { tare }} \\
& =\text { See Calculations Test } 960815 \\
8 \mathrm{H}_{2} \mathrm{Odry} & =\text { See Calculations Test } 960815 \\
8\left(\frac{\Delta \mathrm{W}_{\mathrm{s}}}{\mathrm{W}_{\mathrm{s}}}\right)^{\text {react }} & =100 \frac{\text { gross react }- \text { gross }}{\text { gross }}-\text { tare }_{\mathrm{s}} \\
& =100 \frac{56.69-57.68}{57.68-48.44}=-10.718
\end{aligned}
$$

$\therefore(\text { total volatiles })^{\text {react }}=100-\$$ solids react

$$
\begin{aligned}
& =100-\frac{1}{100}\left[100-8 \mathrm{H}_{2} \mathrm{OD \text {prep }}\right]\left[100+2\left(\frac{\Delta \mathrm{w}_{\mathrm{B}}}{\mathrm{W}_{\mathrm{B}}}\right)^{\text {react }}\right] \\
& =100-\frac{1}{100}[100-17.47][100-10.71] \\
& =26.318
\end{aligned}
$$


Test 960826; Sample U-106, S96T004013

TOTAL SPECIFIC NONCONDENSIBLE GAS PRODUCIION:

$$
\begin{aligned}
\frac{\left(\frac{d P}{d t}\right)_{\text {uncorr }}^{\max }}{w_{s}^{\text {prep }}}= & \frac{0.031}{9.24}=0.0034 \frac{\text { bax }}{\min g^{\text {gred }}} \text { at } 337 \text { to } 396^{\circ} \mathrm{C}, \\
& \text { or } 289-216.5=72.5 \text { min. to } \\
& 297-216.5=80.5 \text { min from onset }
\end{aligned}
$$

By Calculated Graph:

$$
\begin{aligned}
& N_{i}=0.1082 \text { mole, } N_{0}=0.1122 \text { mole, } w_{g}^{\text {prep }}=9.24 \text { grams } \\
& \frac{\Delta N}{W_{s}^{\text {prep }}}=\frac{0.1122-0.1082}{9.24}=4.3 \times 10^{-4} \frac{\text { mole }}{\text { gram prep }} \\
& \frac{\Delta N}{w_{s}^{\text {dry }}}=\frac{\Delta N}{w_{s}^{\text {prep }}} \frac{100-8 \mathrm{H}_{2} \mathrm{O}^{\text {prep }}}{100-8 \mathrm{H}_{2} \mathrm{O}^{\mathrm{dxy}}}=4.3 \times 10^{-4} \frac{100-17.47}{100-44.57} \\
& =6.4 \times 10^{-4} \frac{\text { mole }}{\text { gram dry }} \\
& \frac{\left(\frac{d N}{d t}\right)_{\text {uncorx }}^{\max }}{w_{s}^{\text {prep }}}=\frac{4.6 \times 10^{-5}}{9} \cdot 24=5.0 \times 10^{-6} \frac{\text { mole }}{\text { gram prep }} \\
& \frac{\left(\frac{d N}{d t}\right)_{\text {uncorr }}^{\max }}{w_{s}^{d x y}}=5.0 \times 10^{-6} \frac{100-.17 .47}{100-44.57}=7.4 \times 10^{-6} \frac{\text { mole }}{\text { gram dxy }} \\
& \text { at } 171^{\circ} \mathrm{C} \text { to } 222^{\circ} \mathrm{C} \text { or } \\
& 170-216.5=-46.5 \mathrm{~min} \text { to } \\
& 230-216.5=13.5 \mathrm{~min} \text { from onset }
\end{aligned}
$$


Test 960826; Sample U-106, S96T004013

By Auxiliary Data:

$$
\begin{aligned}
P_{0} & =6.9873 \text { barg, } T_{0}=25.62{ }^{\circ} \mathrm{C}, \quad P_{E}=7.3562 \text { barg, } T_{E}=22.2{ }^{\circ} \mathrm{C} \\
V & =335 \mathrm{~cm}^{3}, \quad R=83.14 \frac{\text { baxa }^{3} \mathrm{~cm}^{3}}{\text { moie }^{\circ} \mathrm{K}} \\
\frac{\Delta N}{W_{s}^{\text {prep }}} & =\frac{1}{W_{s}^{\text {prep }}} \frac{V}{R}\left[\frac{P_{f}+1.01325}{T_{E}+273.15}-\frac{P_{0}+1.01325}{T_{0}+273.15}\right] \\
& =\frac{1}{9.24} \frac{335}{83.14}\left[\frac{7.3562+1.01325}{22.2+273.15}-\frac{6.9873+1.01325}{25.52+273.15}\right] \\
& =6.76 \times 10^{-4} \frac{\text { mole }}{\text { gramprep }} \\
\frac{\Delta N}{W_{s}^{\text {dry }}} & =6.76 \times 10^{-4} \frac{100-17.47}{100-44.57}=1.01 \times 10^{-3} \frac{\text { mole }}{\text { gram dry }}
\end{aligned}
$$

(4) 


\section{WHC-SD-WM-DP-191, REV. I}

Test 960826; Sample U-106, S96T004013

\section{SELF-HEAT PARAMETERS}

$\Delta T_{\text {true }}:$

$$
\begin{aligned}
& T_{i} \approx 210^{\circ} \mathrm{C}, t_{i} \approx 216.5 \mathrm{~min}, \mathrm{~T}_{\theta} \approx 400^{\circ} \mathrm{C}, t_{\theta} \approx 297.7 \mathrm{~min} \\
& \mathrm{~B} \approx 0.87^{\circ} \mathrm{C} / \mathrm{min}, \\
& \Delta T_{\text {txue }}=\phi \Delta T_{\text {corx }}=\phi\left(\left[T_{\theta}-T_{i}\right]-B\left[t_{\theta}-t_{i}\right]\right) \\
& =\phi([400-210]-0.87[297.7-216.5]) \\
& =\phi 119^{\circ} \mathrm{C} \\
& \phi=\frac{\left(\overline{\mathrm{C}}_{\mathrm{ps}}^{\text {react }}\right)\left(\mathrm{w}_{\mathrm{s}}^{\text {Ieact }}\right)+\mathrm{C}_{\mathrm{ph}} \mathrm{w}_{\mathrm{h}}}{\left(\overline{\mathrm{C}}_{\mathrm{ps}}^{\text {react }}\right)\left(\mathrm{w}_{\mathrm{s}}^{\text {react }}\right)}=\left(1+\frac{\mathrm{C}_{\mathrm{ph}} \mathrm{W}_{\mathrm{h}}}{\left(\overline{\mathrm{C}}_{\mathrm{ps}}^{\text {react }}\right) \frac{\mathrm{w}_{\mathrm{s}}^{\text {react }}}{w_{s}^{\text {prep }}} w_{\mathrm{s}}^{\text {prep }}}\right) \\
& =1+\left(\frac{C_{p h} w_{h}}{\left(\bar{C}_{\text {ps }}^{\text {react }}\right) \frac{100+\left(\frac{8}{8} \Delta \frac{w_{s}}{w_{s}}\right)^{\text {react }}}{100} w_{s}^{\text {prep }}}\right) \\
& =1+\left(\frac{0.8368}{\left(\bar{C}_{\mathrm{ps}}^{\text {react }}\right) \frac{100+(-10.71)}{100} 9.24}\right) \\
& =1+\frac{0.101}{\left(\bar{C}_{p s}^{\text {react }}\right)} \quad\left(\left(\bar{C}_{\mathrm{ps}}^{\text {react }}\right) \text { units } \frac{\gamma}{g^{\circ} \mathrm{x}}\right) \\
& \left(\frac{d T}{d t}\right)_{\text {uncorr }}^{\max }=14.0 \frac{{ }^{\circ} \mathrm{C}}{\min } \text { at } 373^{\circ} \mathrm{C} \\
& \text { or } 292.4-216.5=75.9 \mathrm{~min} \text { from onset }
\end{aligned}
$$




\section{WHC-SD-WM-DP-191, REV.}

Test 960826; Sample U-106, S96T004013

THERMAL KINETIC PARAMETERS:

For the initial stage of the runaway, $d T / d t$ vs $Z=-1000 /(T+273.15)$ data for temperatures between $210^{\circ} \mathrm{C}$ and $330^{\circ} \mathrm{C}$ are least-squares fit by the function

$$
\begin{gathered}
\frac{d T}{d t}=B+K_{300} e^{\frac{E_{a}}{R}\left(z-z_{300}\right)}, \\
\text { where } z=-\frac{1000}{T+273.15}, Z_{300}=-\frac{1000}{300+273.15}
\end{gathered}
$$

with parameters $E_{9}=160 \pm 11 \mathrm{KJ} / \mathrm{mole}, \mathrm{K}_{300}=1.39 \pm 0.12{ }^{\circ} \mathrm{C} / \mathrm{min}$; and constants $B=0.87^{\circ} \mathrm{C} / \mathrm{min}$ and $\mathrm{R}=8.3144 \mathrm{~J} / \mathrm{mole} /{ }^{\circ} \mathrm{K}$. 


\section{WHC-SD-WM-DP-191, REV. I}

Test 960826; Sample U-106, S96T004013

\section{SPECIEIC EXOTHERMIC ENERGY RELEASE:}

Neglecting PV work,

$$
\begin{aligned}
& Q \approx \Delta T_{\text {true }}\left(\mathrm{W}_{s}^{\text {react }}\right)\left(\overline{\mathrm{C}}_{\mathrm{ps}}^{\text {react }}\right)=\Delta \mathrm{T}_{\text {corx }} \Phi\left(\mathrm{w}_{\mathrm{s}}^{\text {react }}\right)\left(\overline{\mathrm{C}}_{\mathrm{ps}}^{\text {react }}\right) \\
& \left.=\Delta T_{\text {corr }} \frac{\left[\left(w_{s}^{\text {react }}\right)\left(\overline{\mathrm{C}}_{\mathrm{ps}}^{\text {react }}\right)+\mathrm{w}_{\mathrm{h}} \mathrm{C}_{\mathrm{ph}}\right]}{\left(\mathrm{w}_{\mathrm{s}}^{\text {react }}\right)\left(\overline{\mathrm{C}}_{\mathrm{ps}}^{\text {react }}\right)}{ }_{\text {react }}\right)\left(\overline{\mathrm{C}}_{\mathrm{ps}}^{\text {react }}\right) \\
& =\Delta T_{\text {corr }}\left[\left(w_{\mathrm{s}}^{\text {react }}\right)\left(\overline{\mathrm{C}}_{\mathrm{ps}}^{\text {react }}\right)+\mathrm{w}_{\mathrm{h}} \mathrm{C}_{\mathrm{ph}}\right] \\
& \hat{Q}_{\mathrm{dry}} \approx \Delta \mathrm{T}_{\text {corr }}\left[\frac{\mathrm{w}_{s}^{\text {react }}}{\mathrm{w}_{\mathrm{s}}^{\text {dry }}}\left(\overline{\mathrm{C}}_{\mathrm{ps}}^{\text {react }}\right)+\frac{\mathrm{w}_{\mathrm{h}} \mathrm{C}_{\mathrm{ph}}}{\mathrm{w}_{\mathrm{s}}^{\text {dry }}}\right]
\end{aligned}
$$

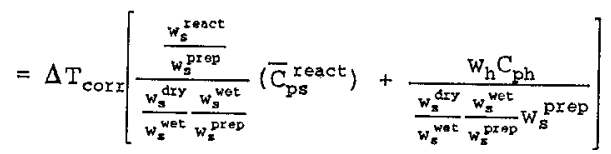

$$
\begin{aligned}
& \left.=\Delta T_{\operatorname{cor} x} \frac{\frac{w_{s}^{\text {prep }}}{\frac{w_{s}^{\text {wet }}}{w_{s}^{\text {dry }}}}\left[\frac{w_{s}^{\text {react }}}{w_{s}^{\text {wet }}}\right.}{w_{s}^{\text {prep }}}\left(\bar{C}_{p s}^{\text {react }}\right)+\frac{w_{h} C_{p h}}{w_{g}^{\text {prep }}}\right] \\
& \hat{Q}_{\mathrm{dxy}} \approx \Delta \mathrm{T}_{\operatorname{cor} x} \frac{\left(100-8 \mathrm{H}_{2} \mathrm{O}^{\mathrm{prep}}\right)}{\left(100-8 \mathrm{H}_{2} \mathrm{O}^{\mathrm{dxy}}\right)}\left[\frac{100+\left(\frac{8}{\mathrm{sw}_{z}}\right)^{\text {react }}}{100}\left(\overline{\mathrm{C}}_{\mathrm{ps}}^{\text {react }}\right)+\frac{\mathrm{w}_{\mathrm{h}} \mathrm{C}_{\mathrm{ph}}}{\mathrm{w}_{\mathrm{s}}^{\text {prep }}}\right] \\
& =119 \frac{(100-17.47)}{(100-44.57)}\left[\frac{100+(-10.71)}{100}\left(\bar{C}_{\mathrm{ps}}^{\text {yeact }}\right)+\frac{0.8368}{9.24}\right] \\
& =158.2\left(\overline{\mathrm{C}}_{\mathrm{ps}}^{\text {react }}\right)+16.0 \frac{\mathrm{J}}{\mathrm{g}^{d x y}}
\end{aligned}
$$


WHC-SD-WM-DP-191, REV. 1

SAMPLE DATA SUMMARY

65 
WHC-SD-WM-DP-191, REV. 1

THIS PAGE WAS INTENTIONALLY LEFT BLANK

66 


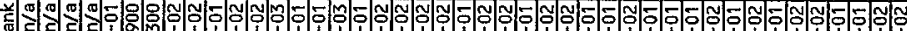

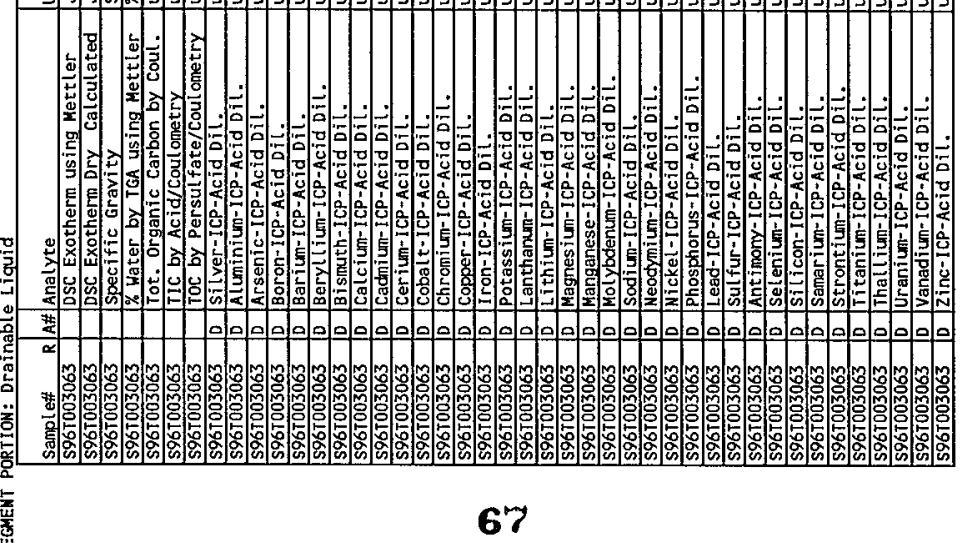


i

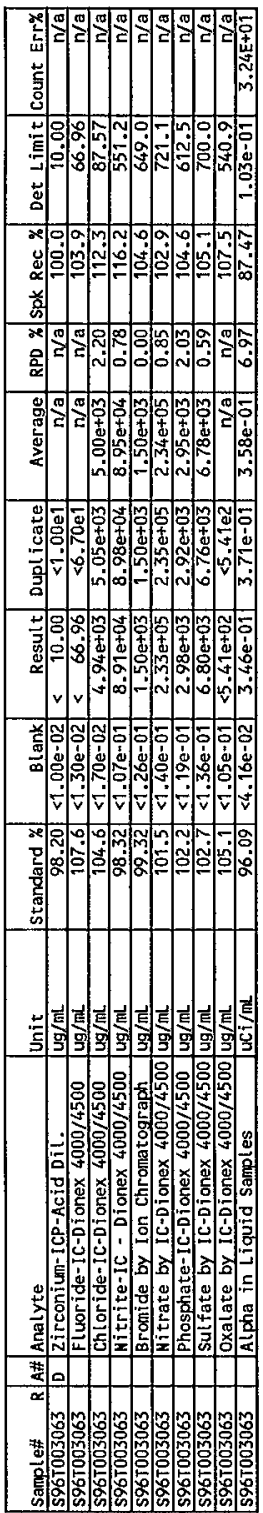

$\stackrel{\circ}{\circ}$

웅 


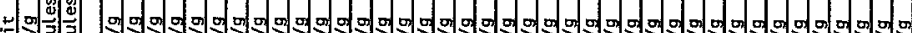

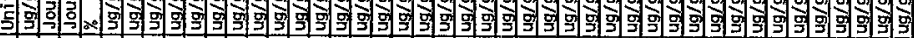

단 $>$

의

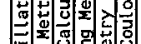

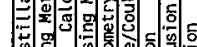

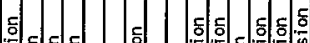

등 등

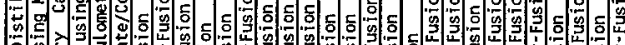

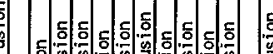

드는

息 至

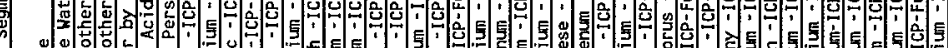

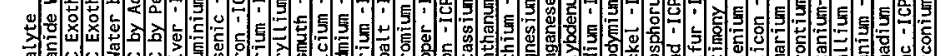

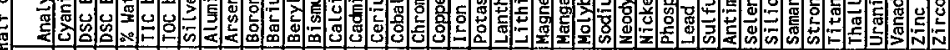

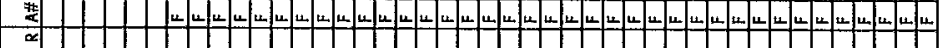



。

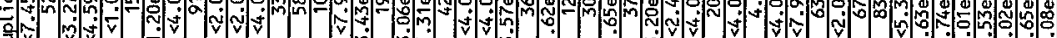
官.

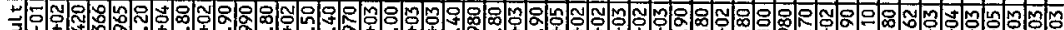

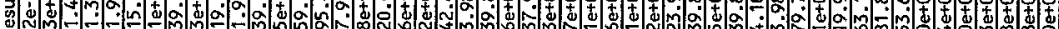

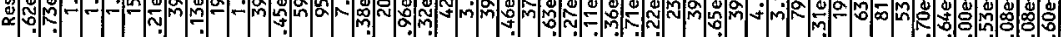
- -

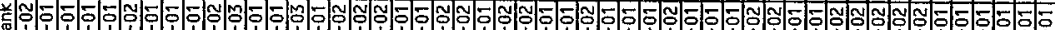

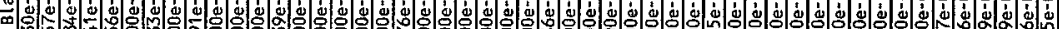
m.

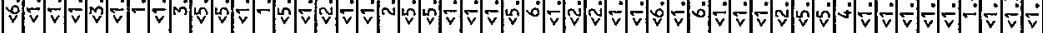
大

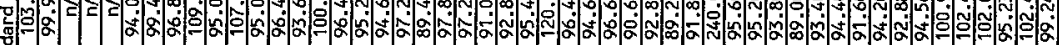
悹

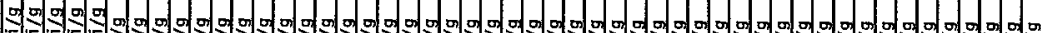

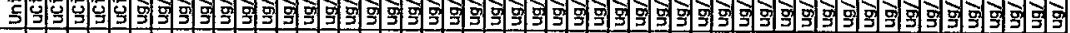

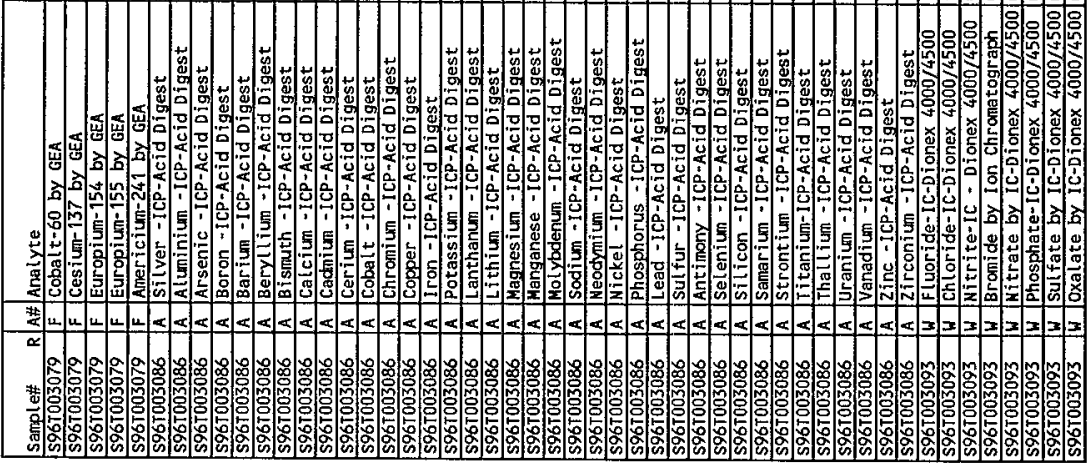




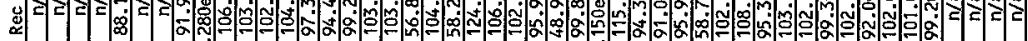
: के

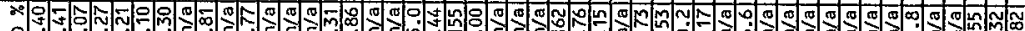

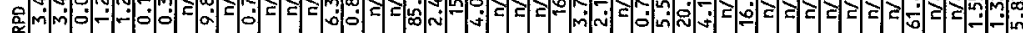

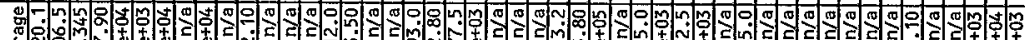

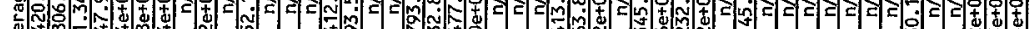
?

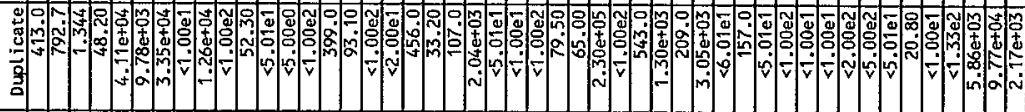

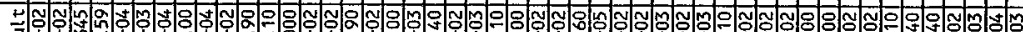

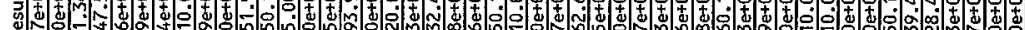
a N

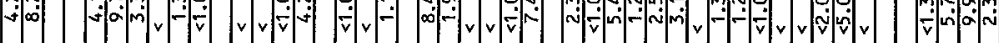

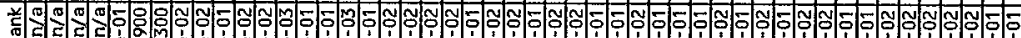

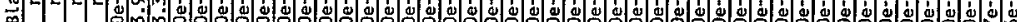
$\infty$

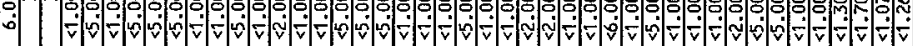

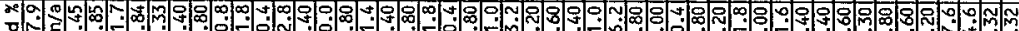
둥 案

प्र

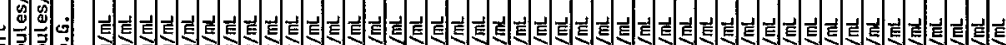

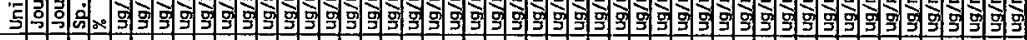

这营

$\rightarrow$ ज

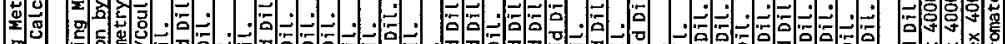
명

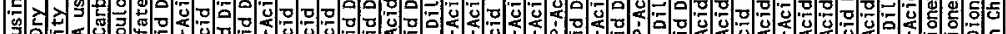
目国 ⿹勹巳 4 苟

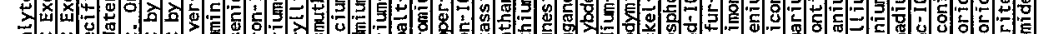

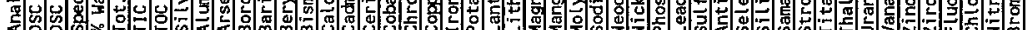

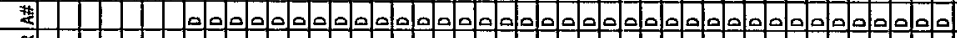


和

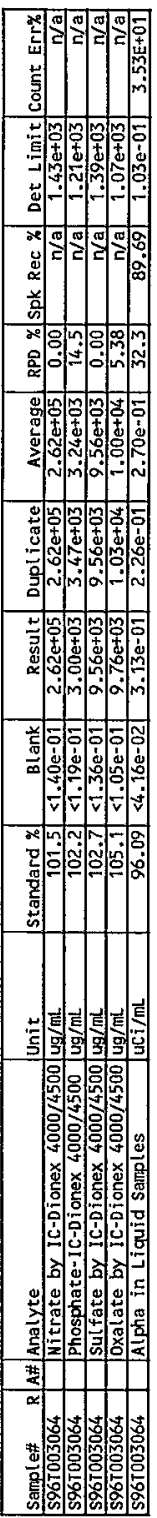




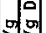

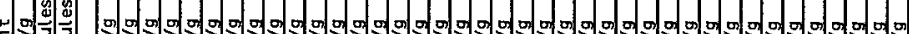

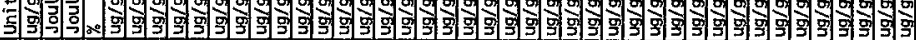

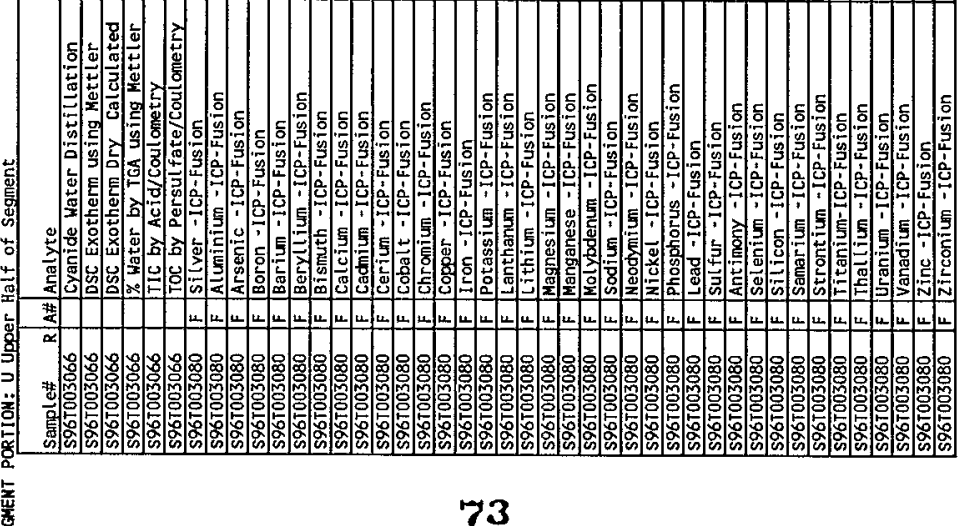




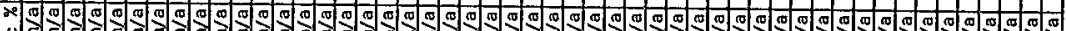

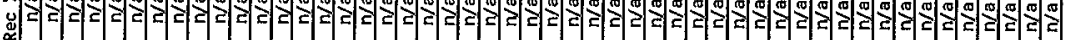
学 a

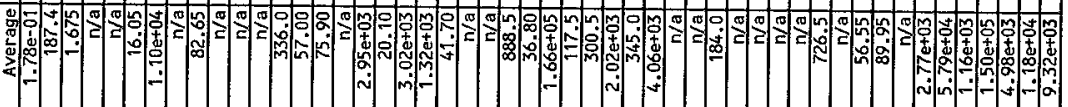

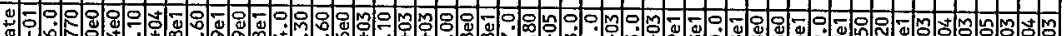
g

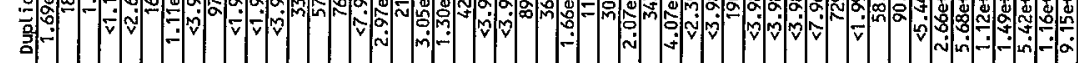

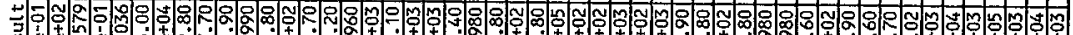

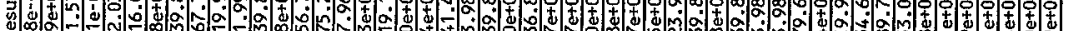

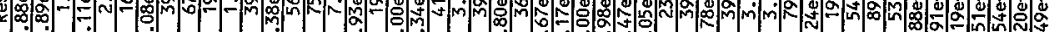

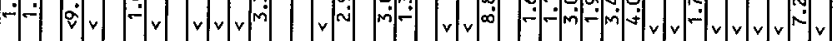

菏:

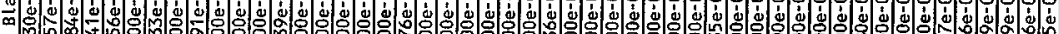

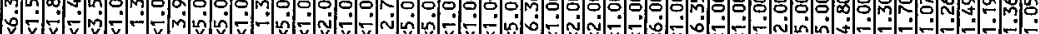

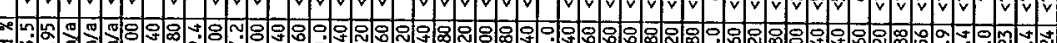

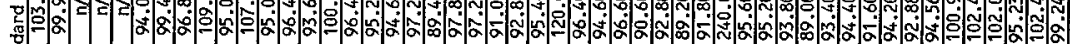
密

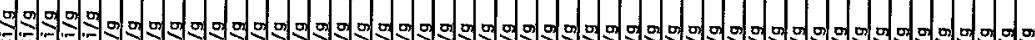

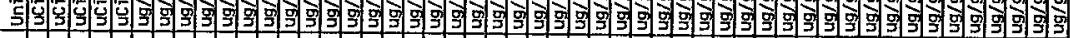

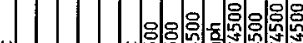

(

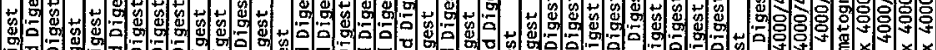

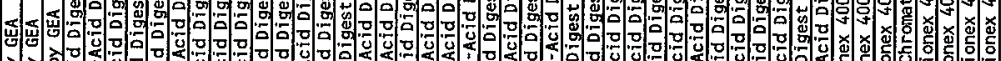

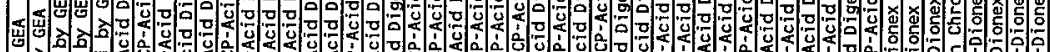

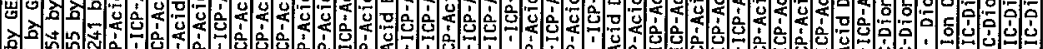

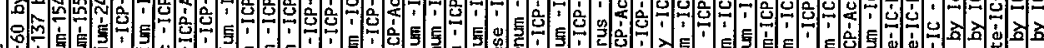

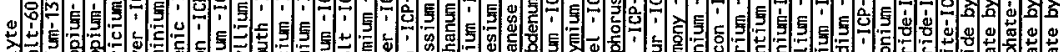

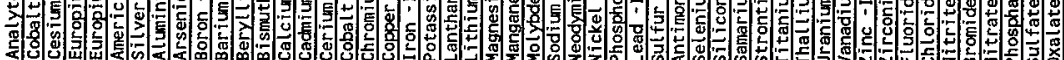

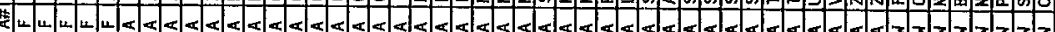

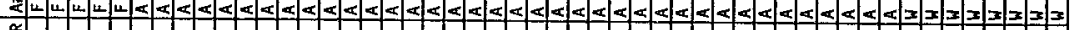




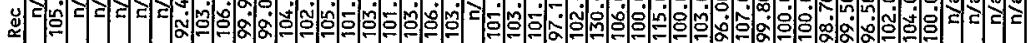
:

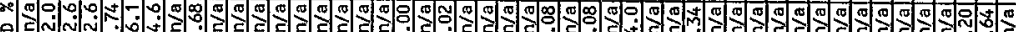

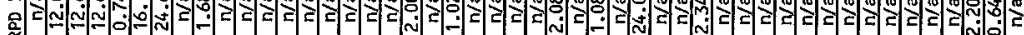

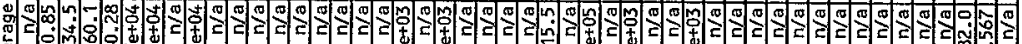

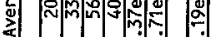

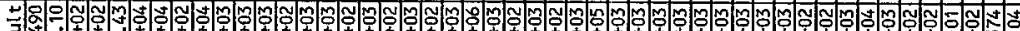

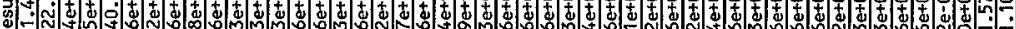

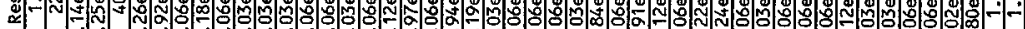

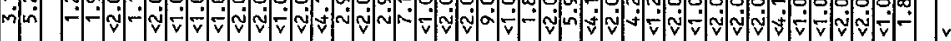

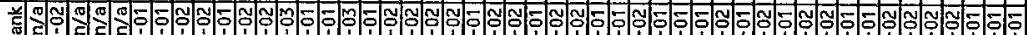

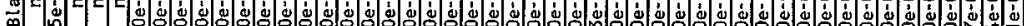
N

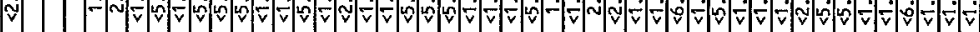

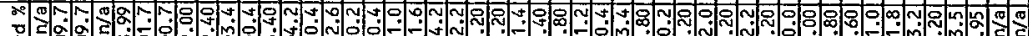

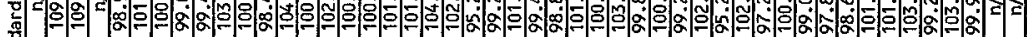
홇

12 空

$+10$.

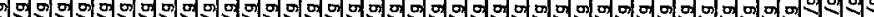

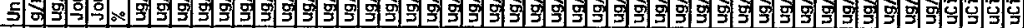

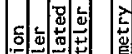

.

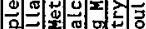

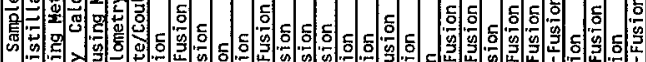

4

$>$ 司

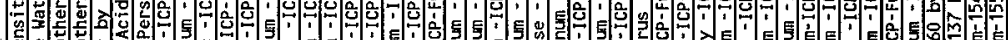
4 到

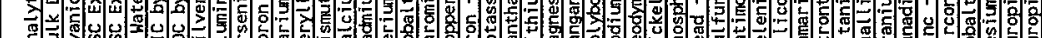

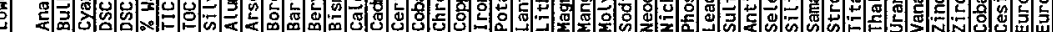




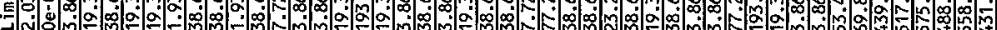
范

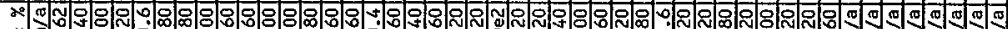

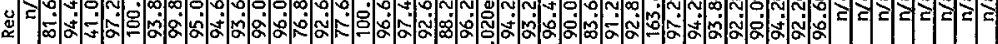
总

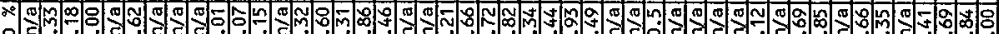

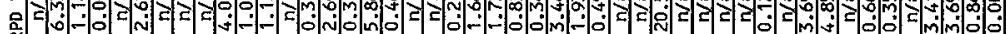

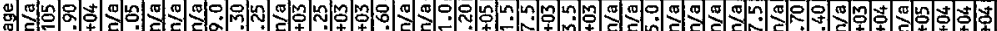

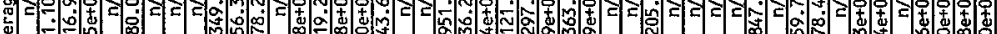

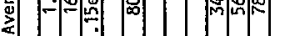

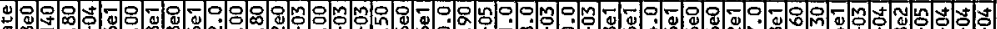

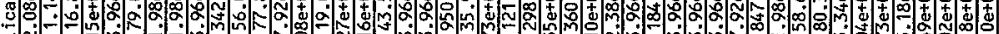
今

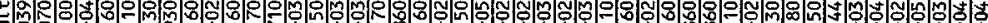

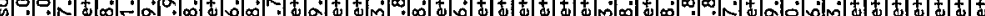

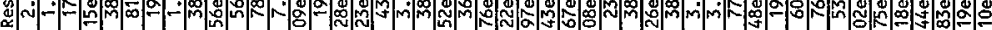

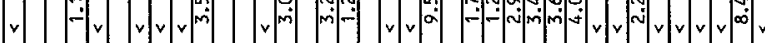

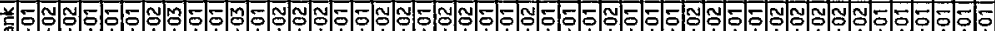

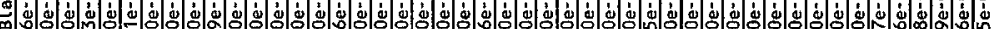
n- mo b

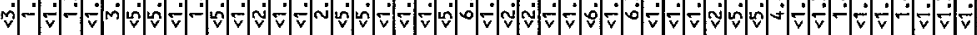

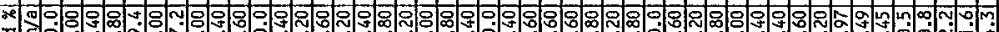

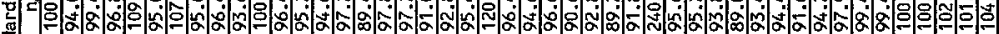
帝

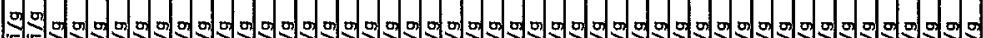

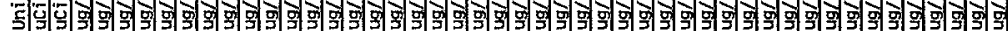

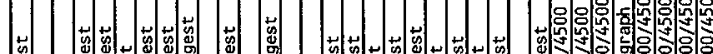
0 荡

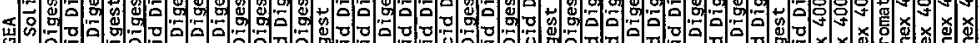

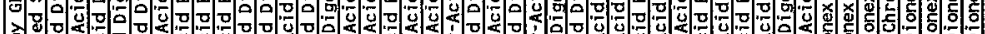
1

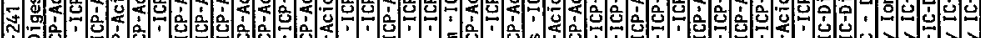
点 过

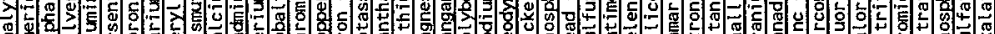

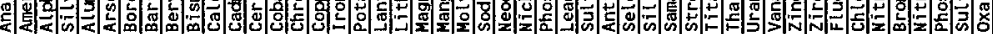

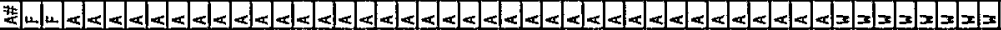

กิ

$\ddot{*}$ 
容

ㅇํำ.

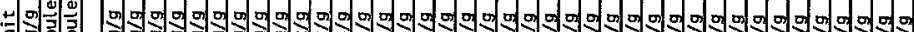
5

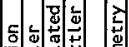

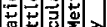

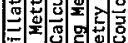

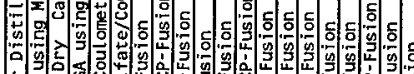

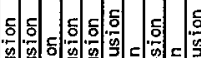

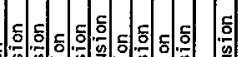




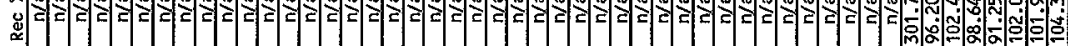
㮍

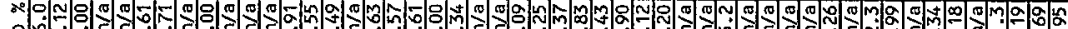

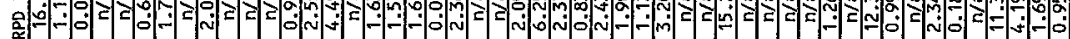

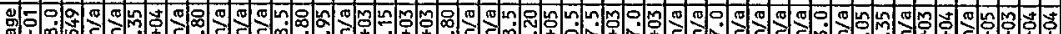
웅

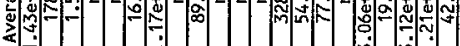

$4-0$-0 (1)

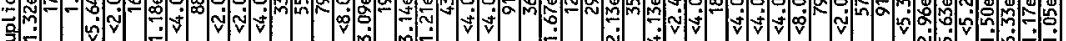

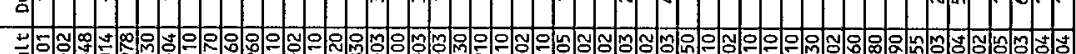

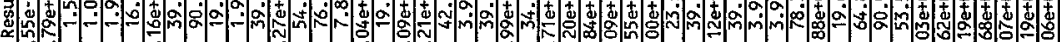

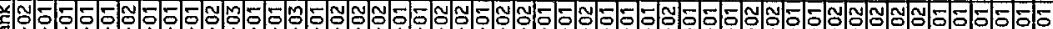

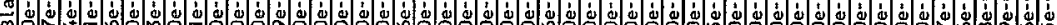

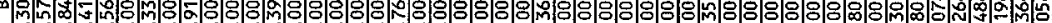

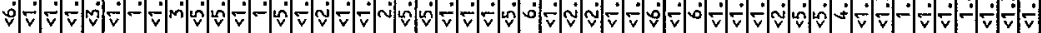
x"n. 迸 는

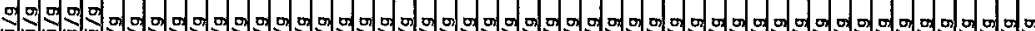
들

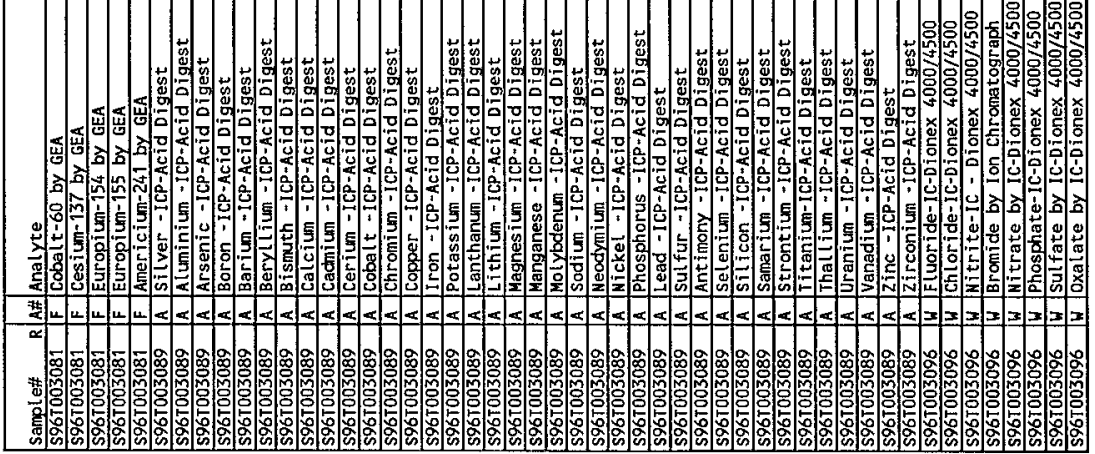

$\stackrel{\circ}{\circ}$ 
WHC-SD-WM-DP-191, REV.I

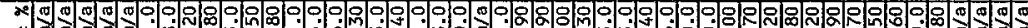

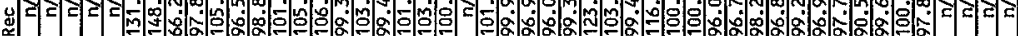
总

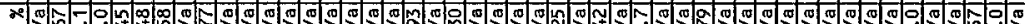

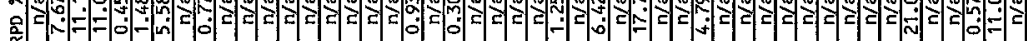

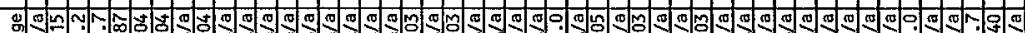

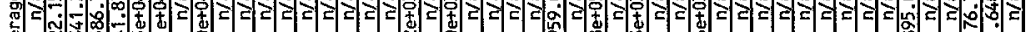

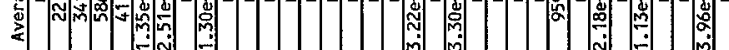

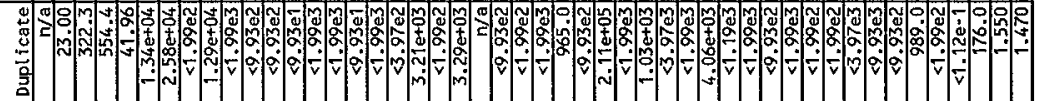

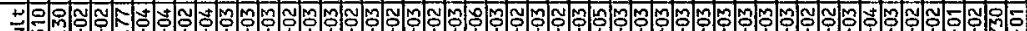

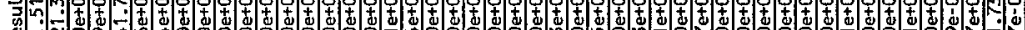
- No

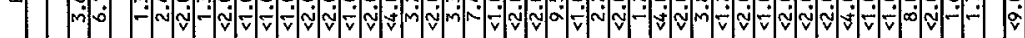

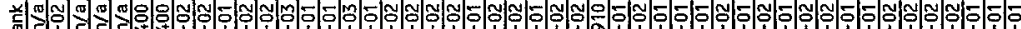

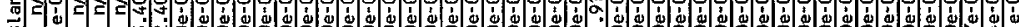

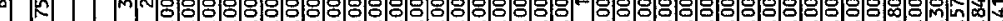

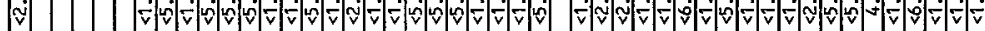

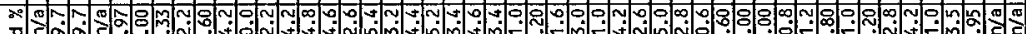

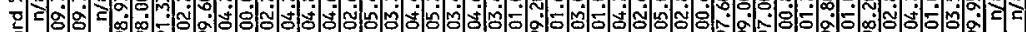
늄 离

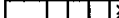

영요

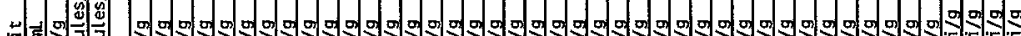

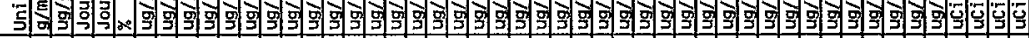

둥

1.

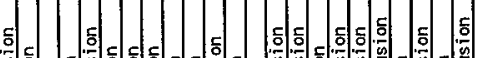

氧司国 क 4 (5)

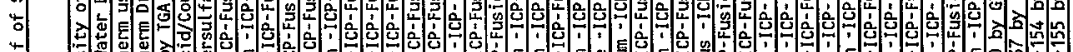

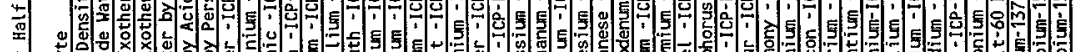

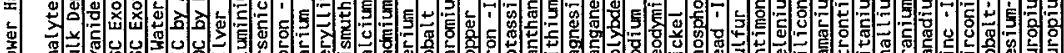
美 


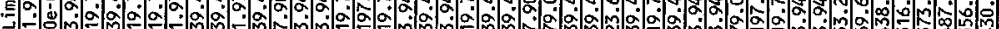

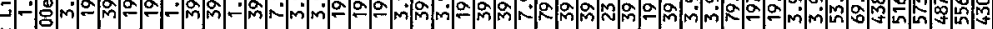
㟧

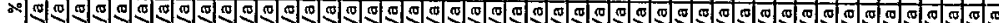

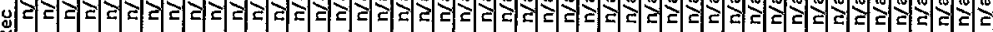
흥

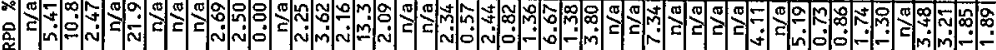
y. 递

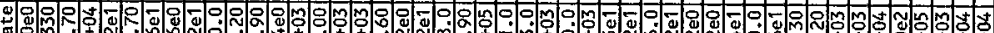
:

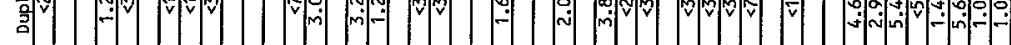

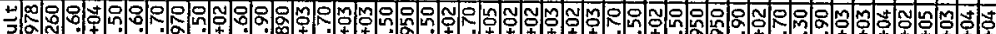

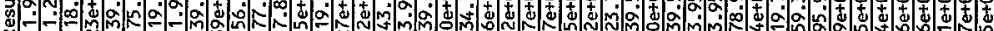

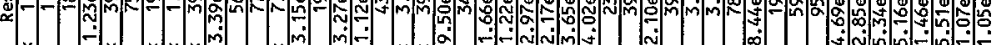

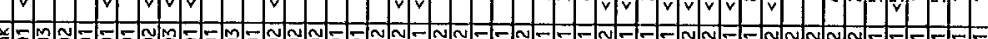

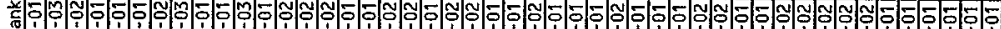

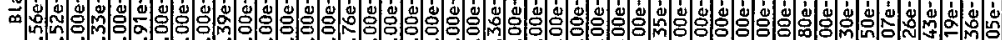

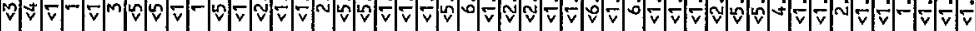
x

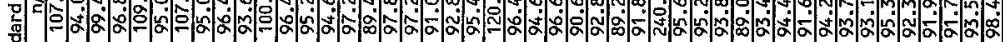
空

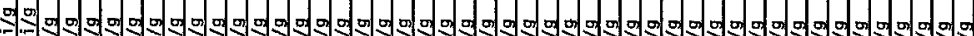

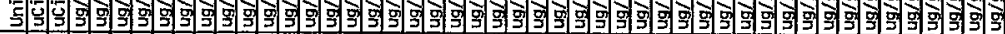

$\checkmark$ च -

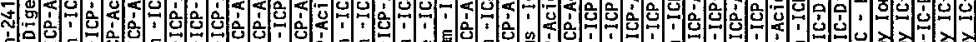

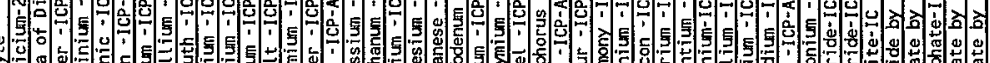
일.

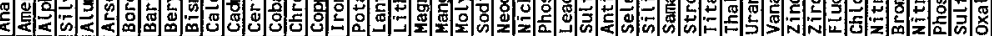

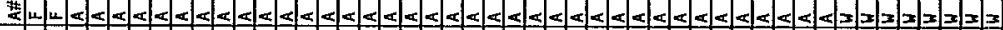
a 
WHC-6D-WM-DP-191, REV.I

$\stackrel{\infty}{=}$

$\underset{0}{\ddot{g}}$

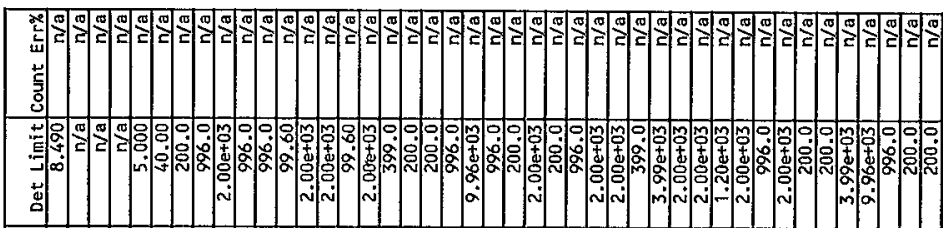

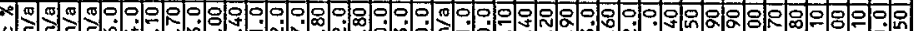

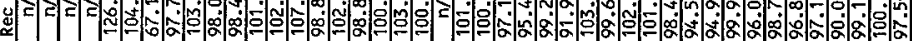

幽

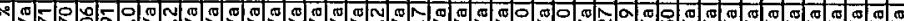

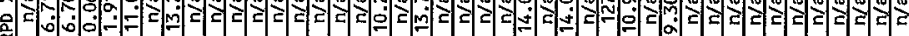
बठ

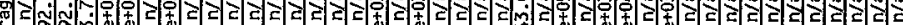
\.

营

요

\% m

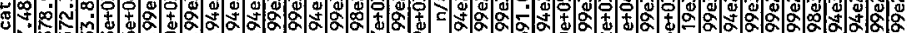

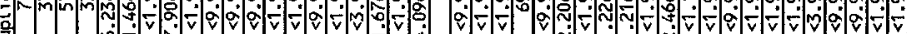

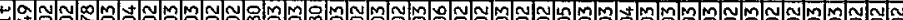
当

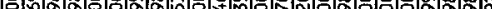

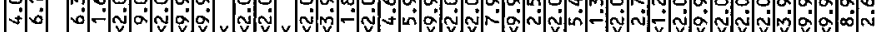
落

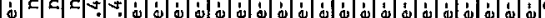
业

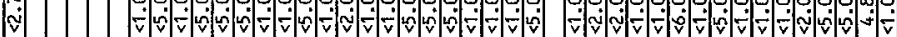
x) 눙 प्रे 峘

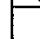

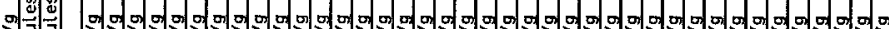

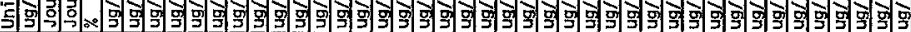

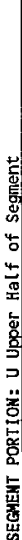

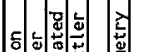$$
\text { 군뭉 }
$$

可矛这

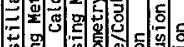

든

등 등

당듸드

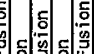

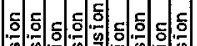

등

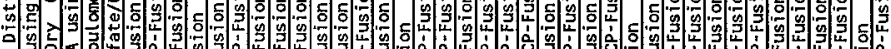

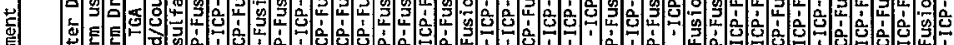

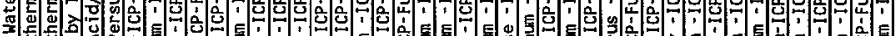

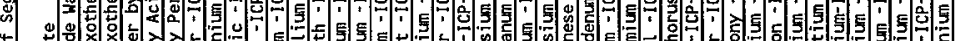

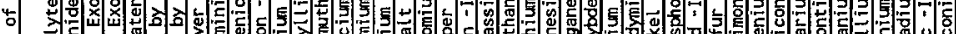

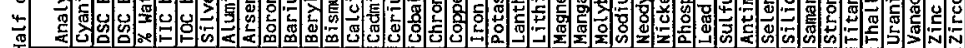

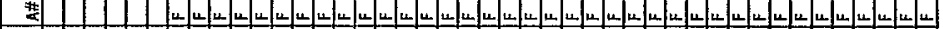

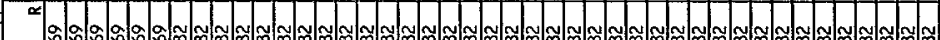
* 


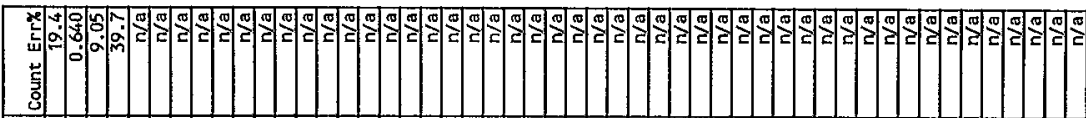

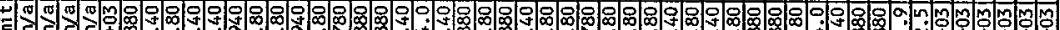

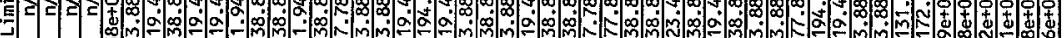

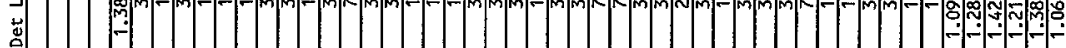

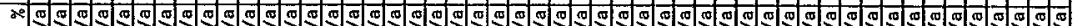

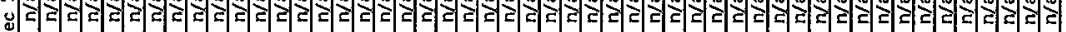
苨

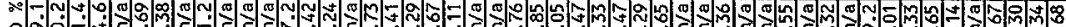

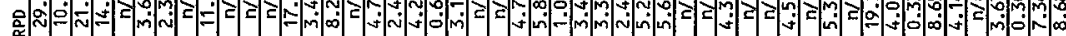

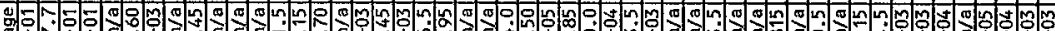

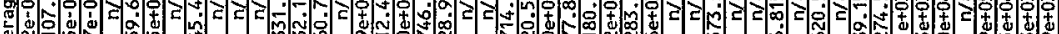

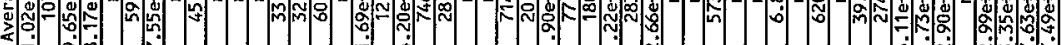

궁- -

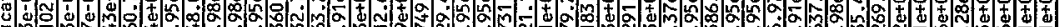
等

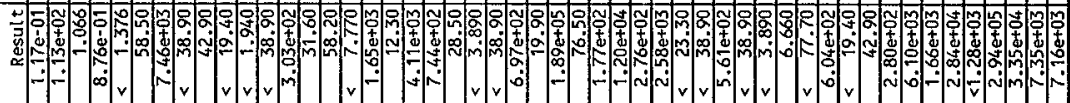

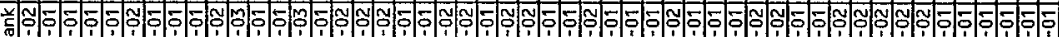
-

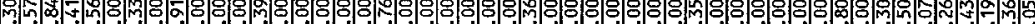

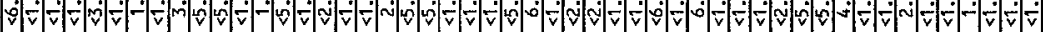

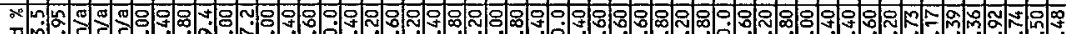
? 密

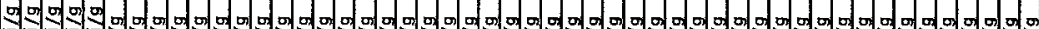

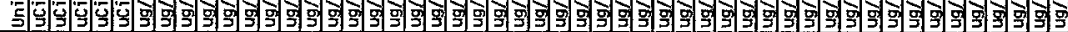

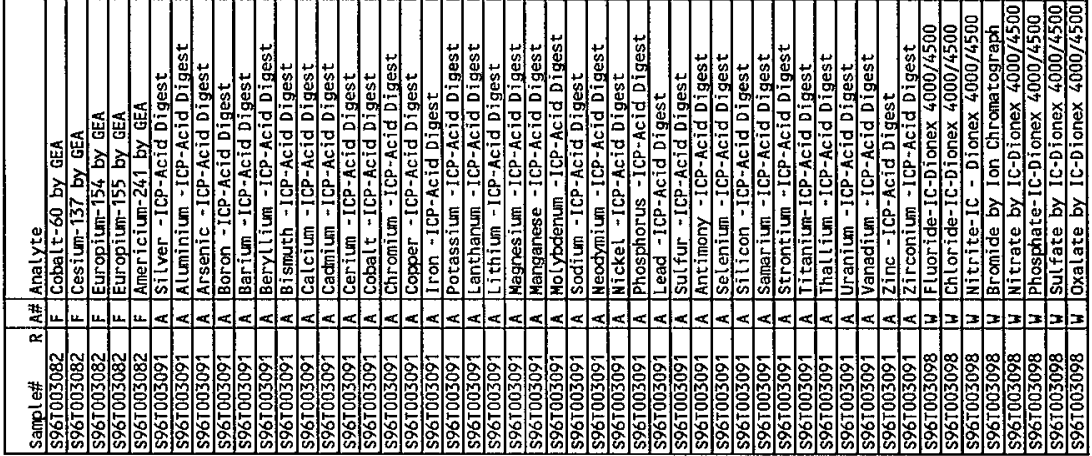
$\stackrel{2}{2}$ 


\section{WHC-SD-WM-DP-191, REV. I}

오

$\ddot{g}$

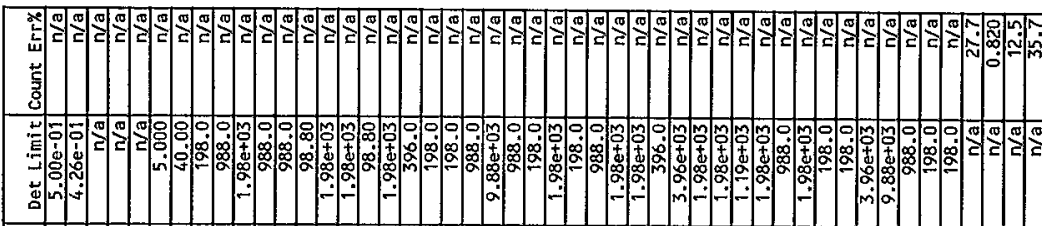

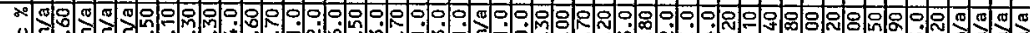
级 苋

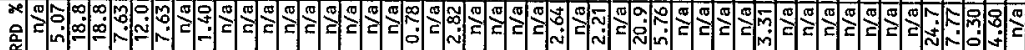

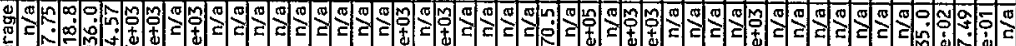

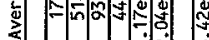
mi

. :

\$

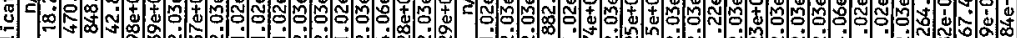

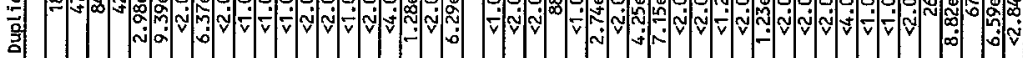

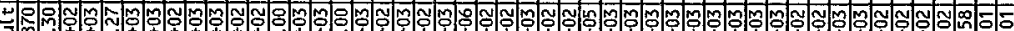
马一由

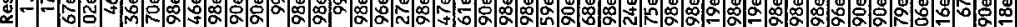

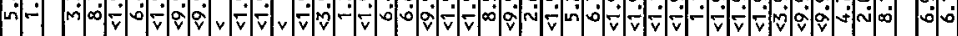

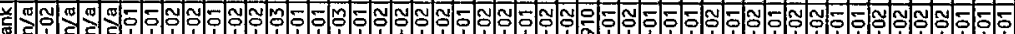
包 Na

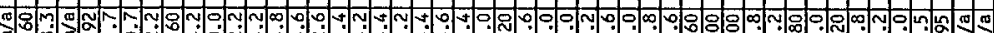
궁

产

110

可

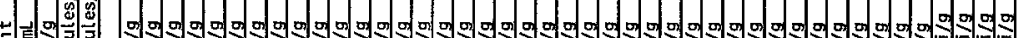

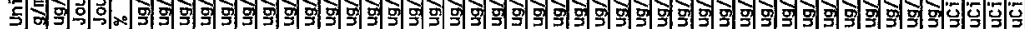

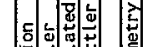

ง

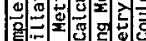

눙

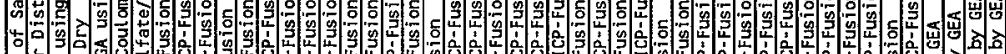

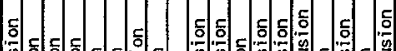

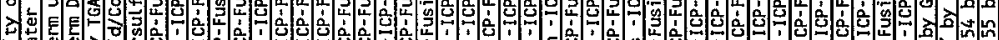

"⿹丁口

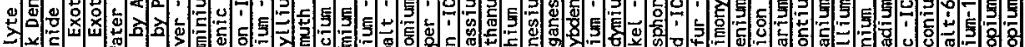
홍 궁

葙 


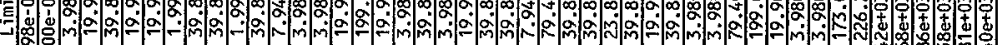
$+0$. $\Delta N$

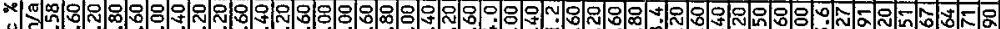

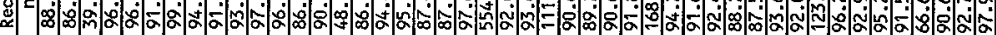
㚇

20 ॠ

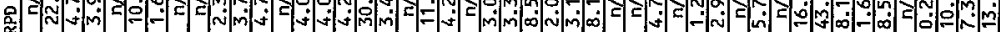
40.

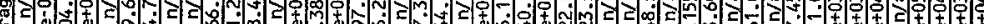
年

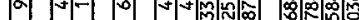

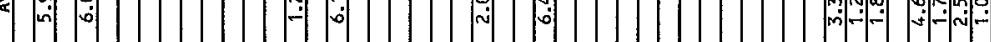

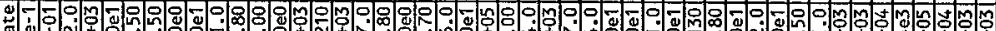

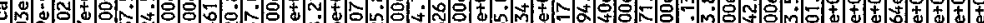

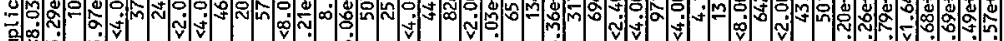

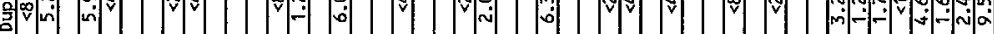

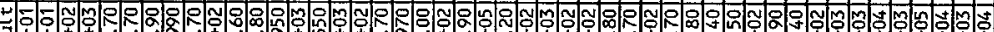
司造

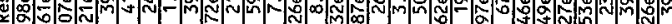

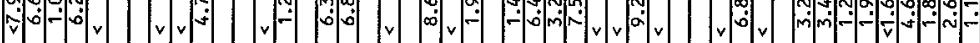

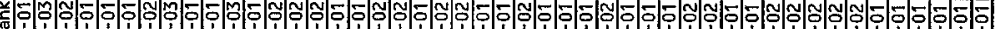
๓

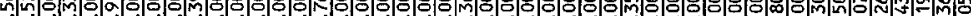

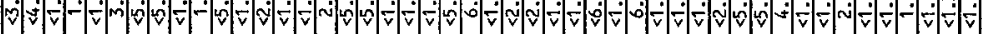

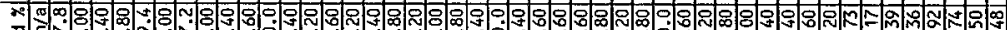
망 प्ञ

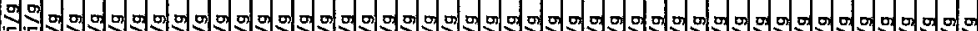

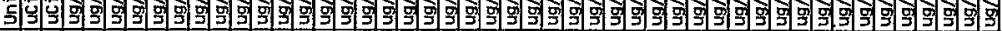

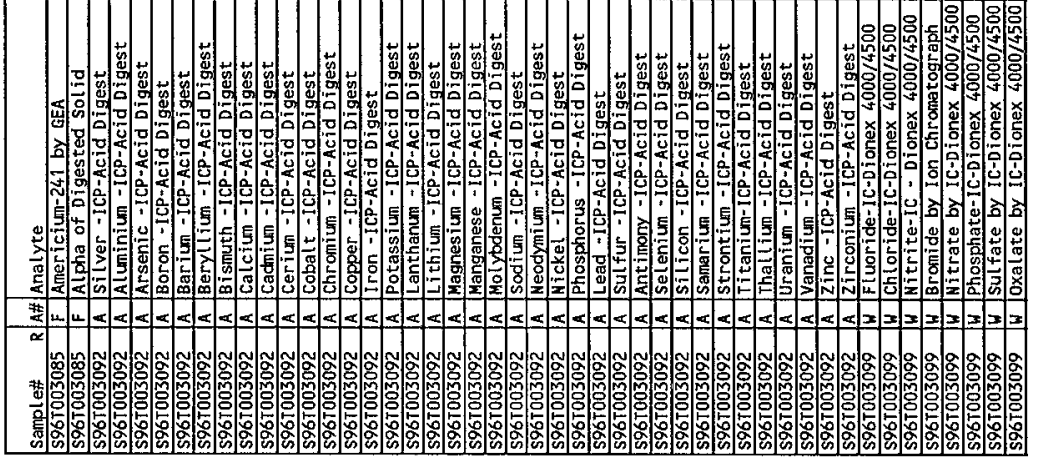

$\stackrel{\circ}{\circ} m$ 
$\underset{\ddot{j}}{\ddot{D}}$

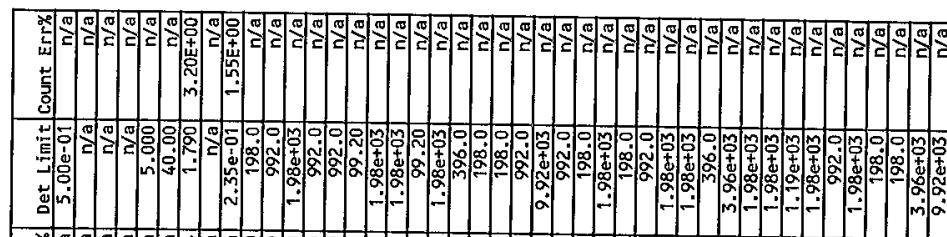

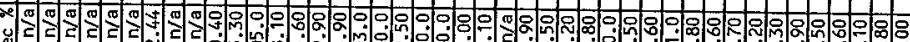

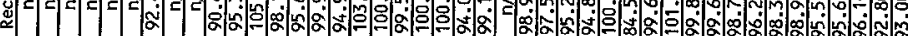
苛

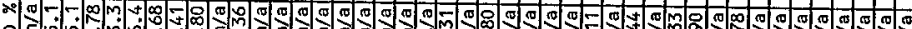
을 :

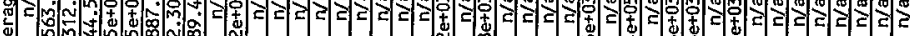
\& $\therefore$

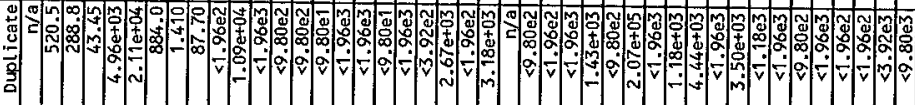

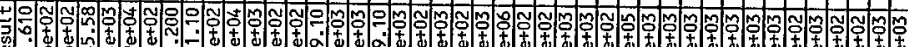

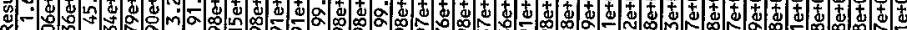
in

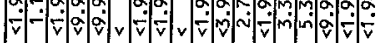

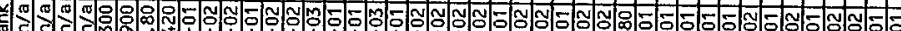
7 궁

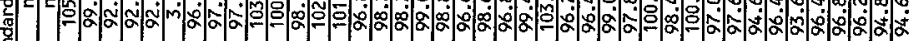

숭

의

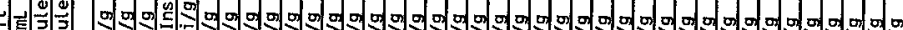

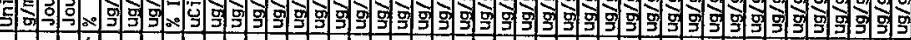

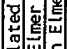

4.

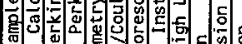

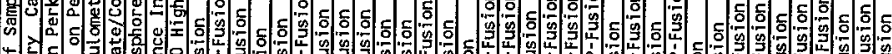

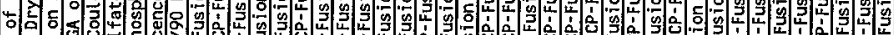

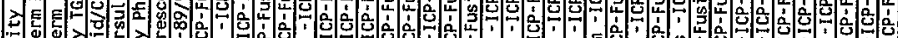

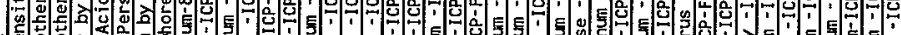

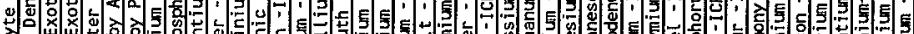




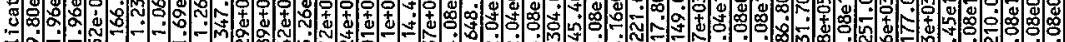

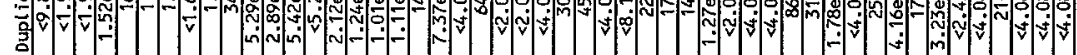

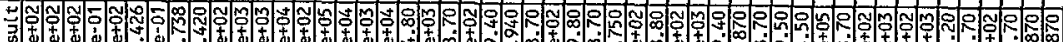

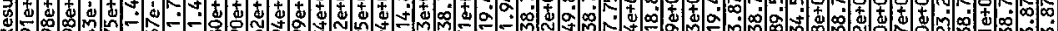

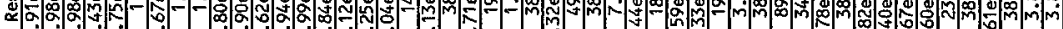

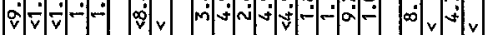

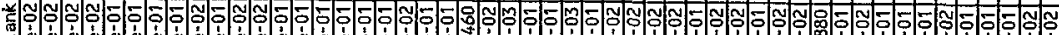

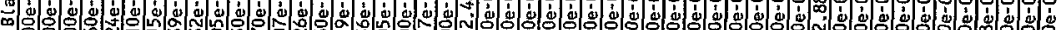
on

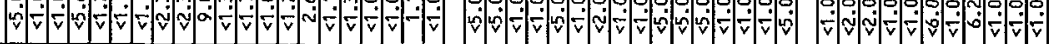
20. की

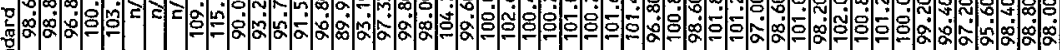
密

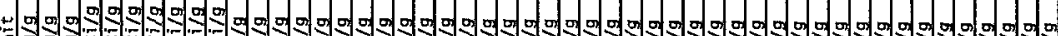

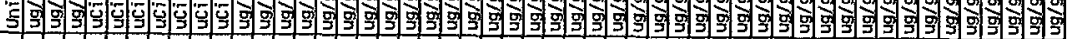

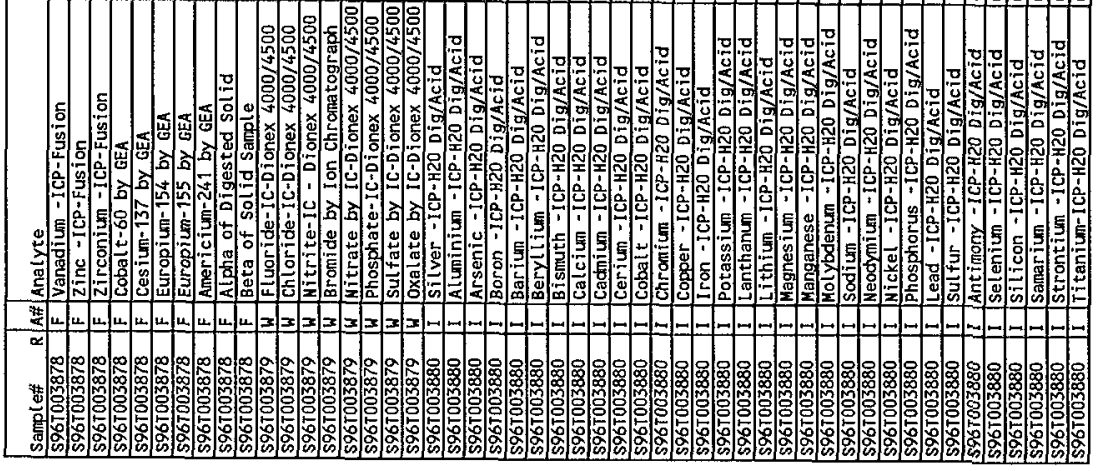
$2 m$ 

至 $\checkmark \bar{v} \vee$ $\checkmark$ in

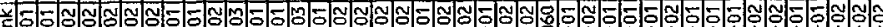

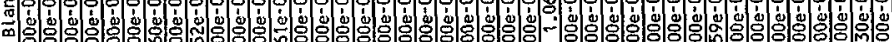

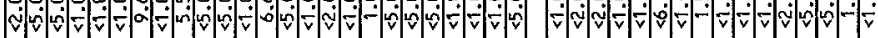
x

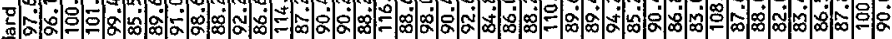
옳

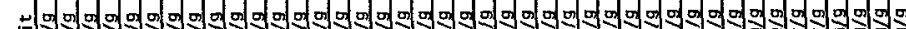

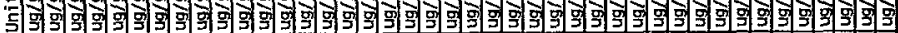

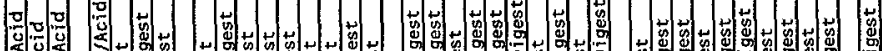

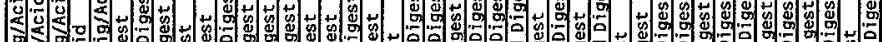

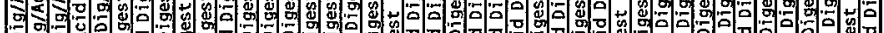
10

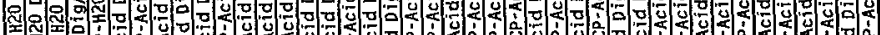

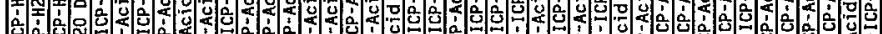

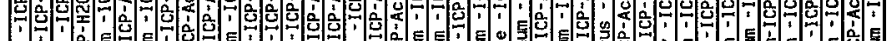

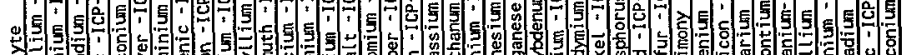

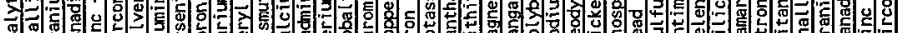

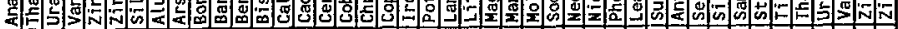

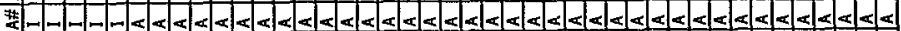

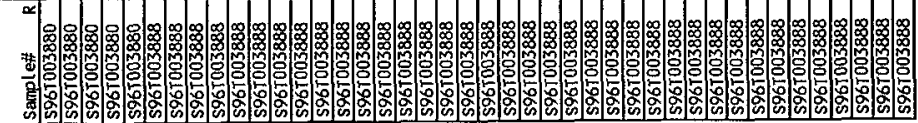


$\underset{a}{a}$

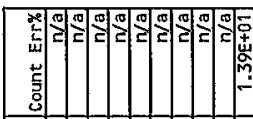

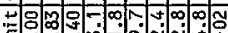

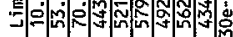

Ф

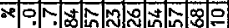

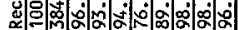

현

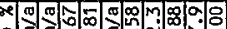

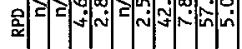

:

₹

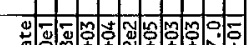

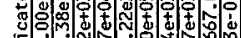

言v $v$ a

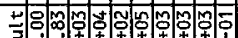

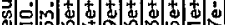

$\approx$ N

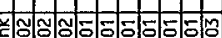
(1)

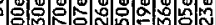
$\div \div \div \div \div \div \div$

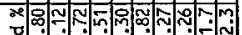

co

窇

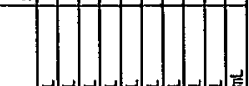

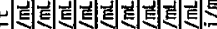

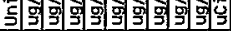

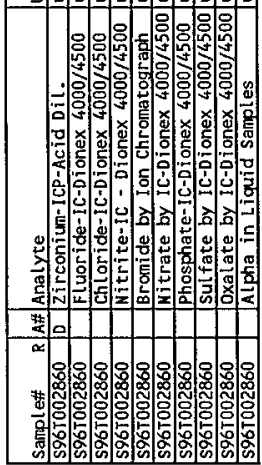

¿

형 
WHC-SD-WM-DP-191, REV.I

$\hat{N}$

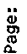

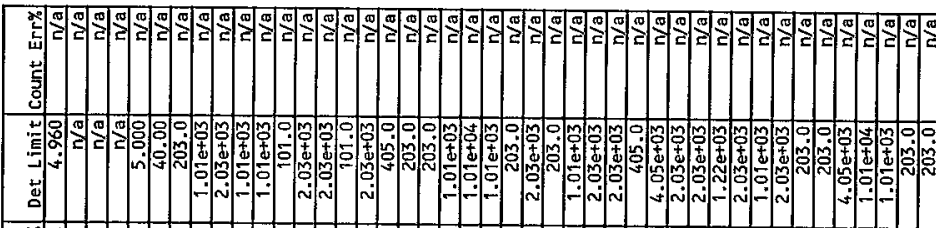

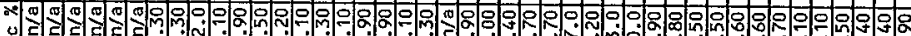

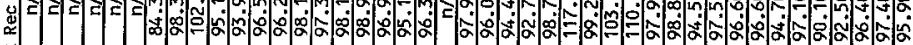
劳

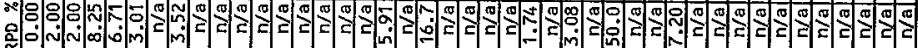

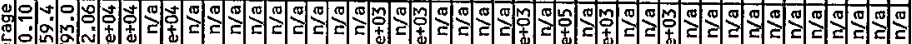

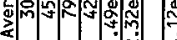

蒠

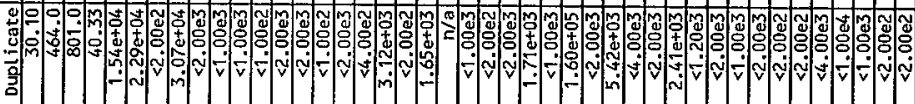

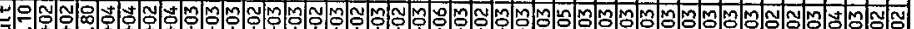

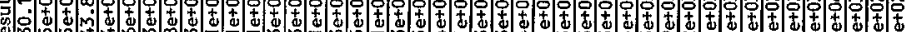

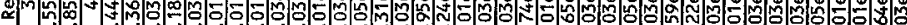

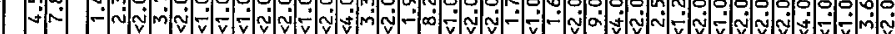

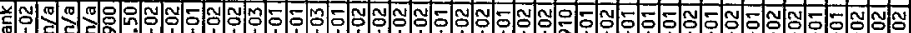
-0 N

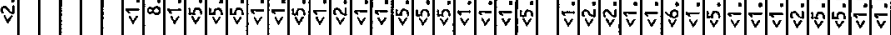

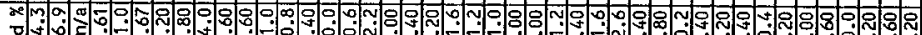

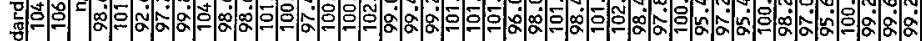
空

(c)

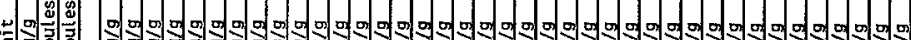

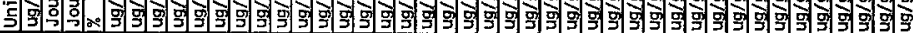

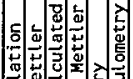

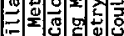

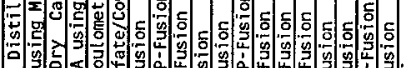

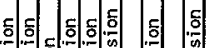

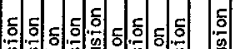

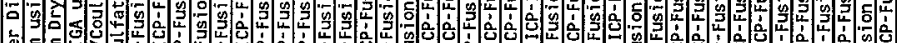
包

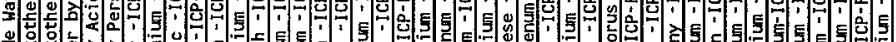

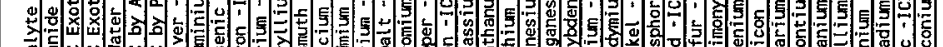

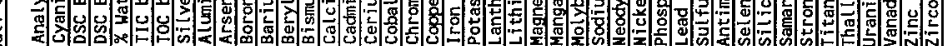

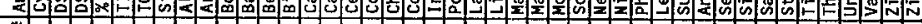

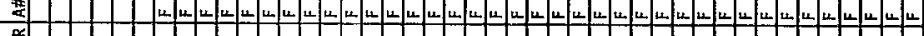




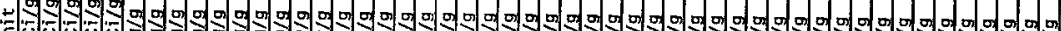

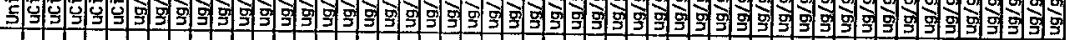

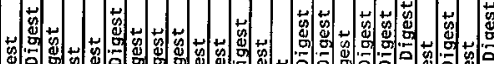

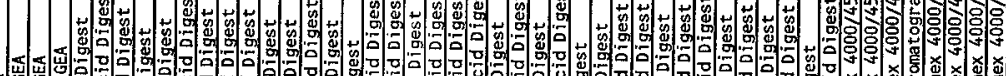

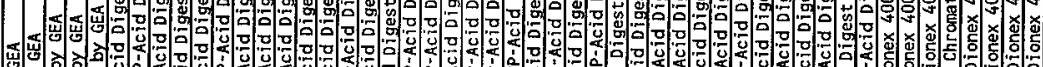

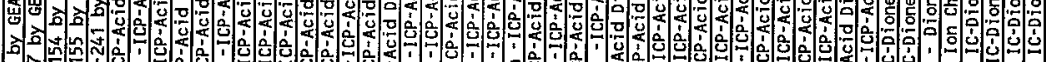

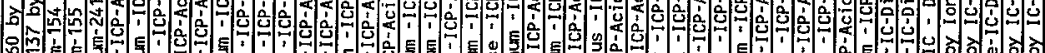
40 至

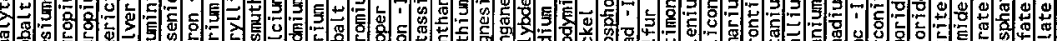

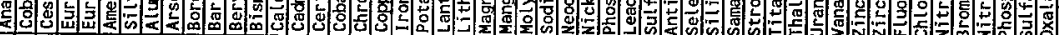
烈

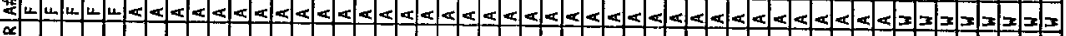


WHC-SD-WM-DP-191, REV.I

ì

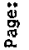

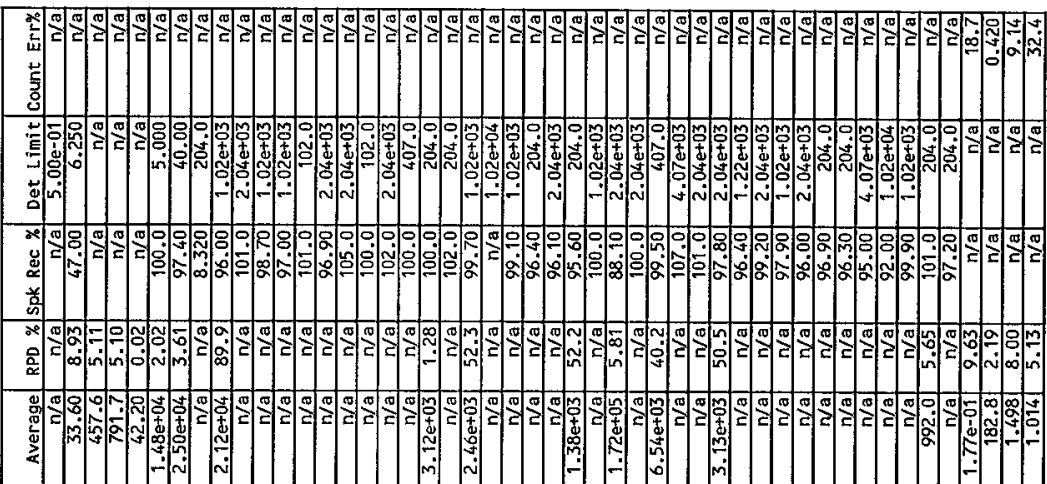

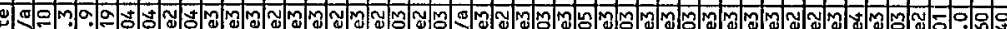
. 咅

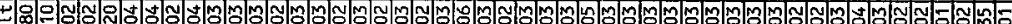

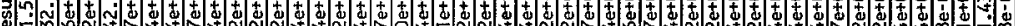
ڤ

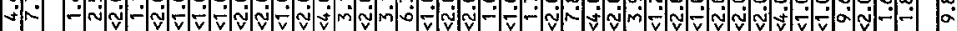

*0.

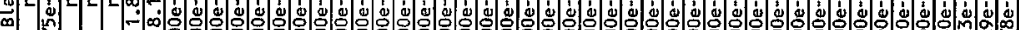

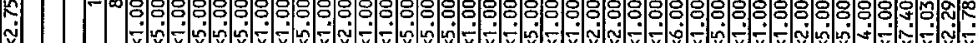

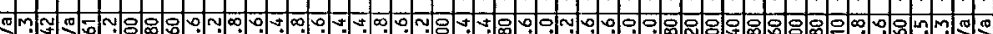

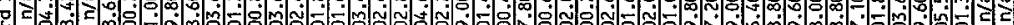

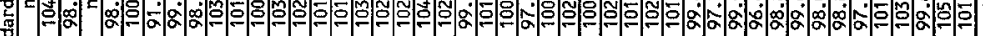
대용 s

ar

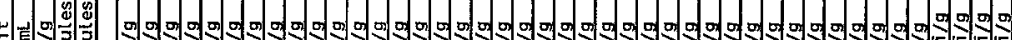

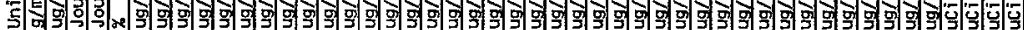

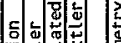

0.0

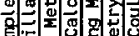

영

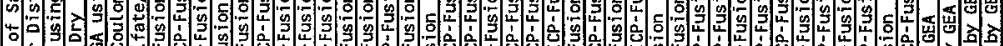

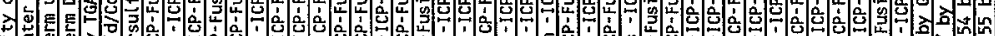

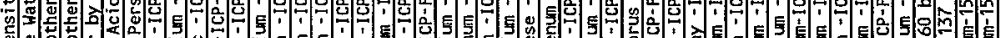

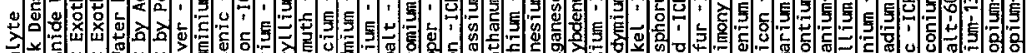

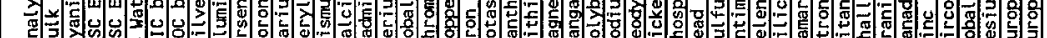

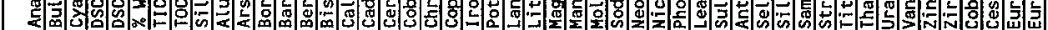

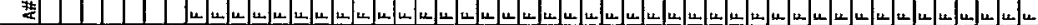

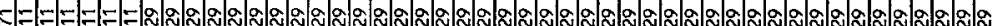




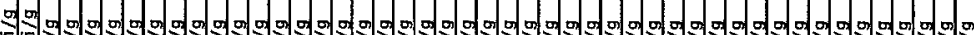

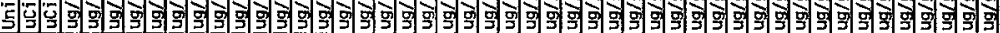

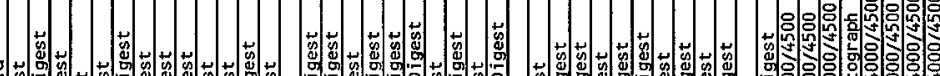
出 3 包

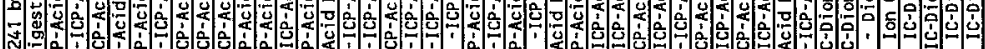

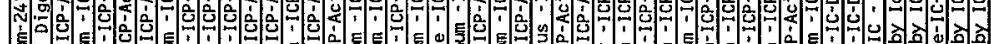

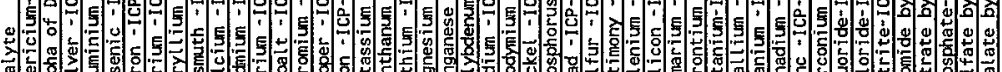
둔

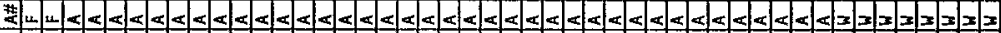


20. ज。

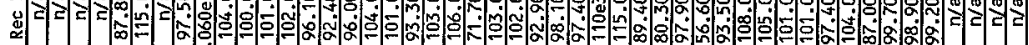
$\stackrel{*}{*}$ ,

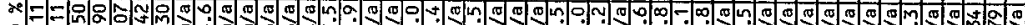

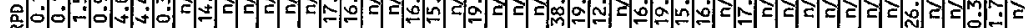

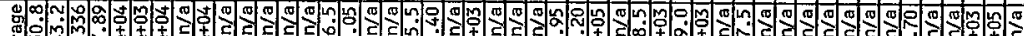

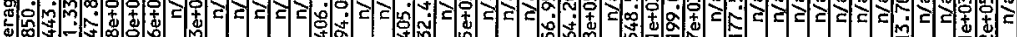

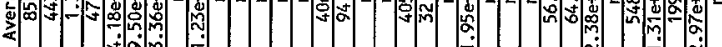

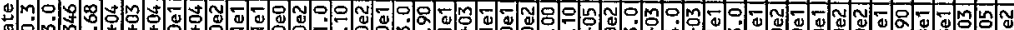

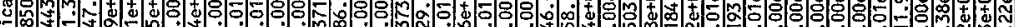

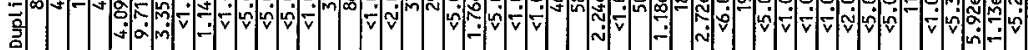

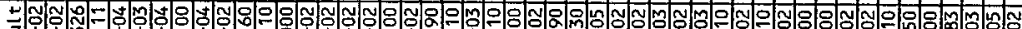

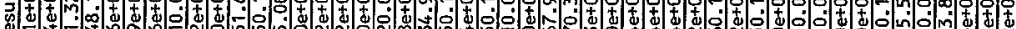

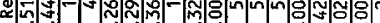

$\infty$

$\checkmark a^{\circ} m$

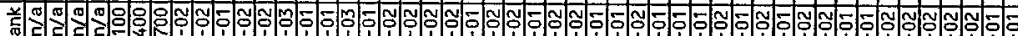

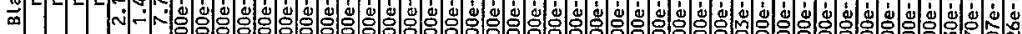

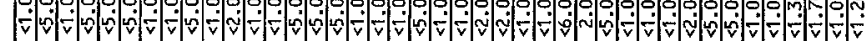
- a

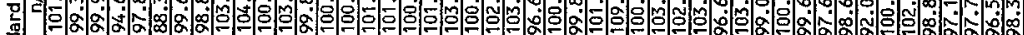
突

군

며응

(5)

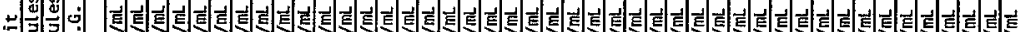

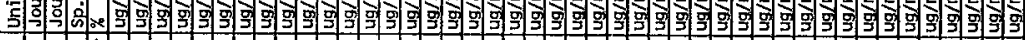

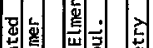

魚 至

국ㅂ. 든

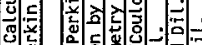

a c

:

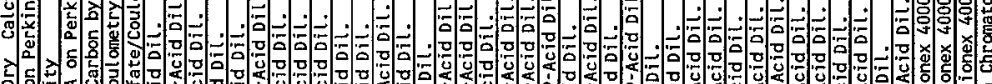

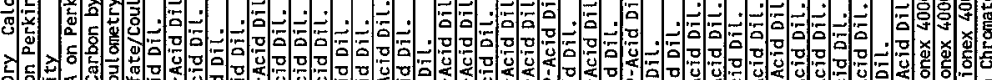

- 영ㅇㅇㅇㅎㅇ

이요대다

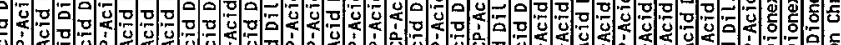

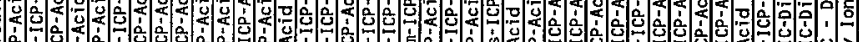

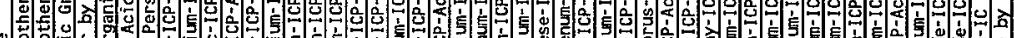

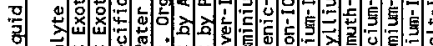

勿

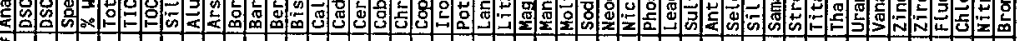

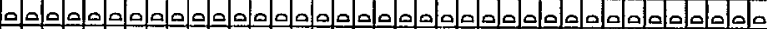

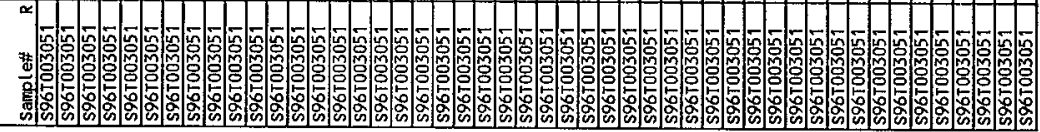


กั

兽

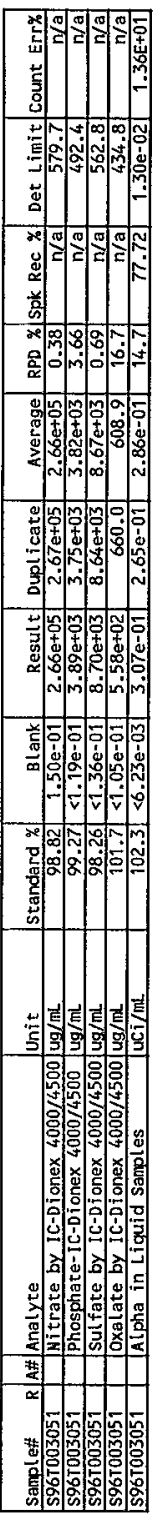

․

웡 


\section{WHC-3D-WM-DP-191, REV.I}

m
$\ddot{u}$
0
0
0

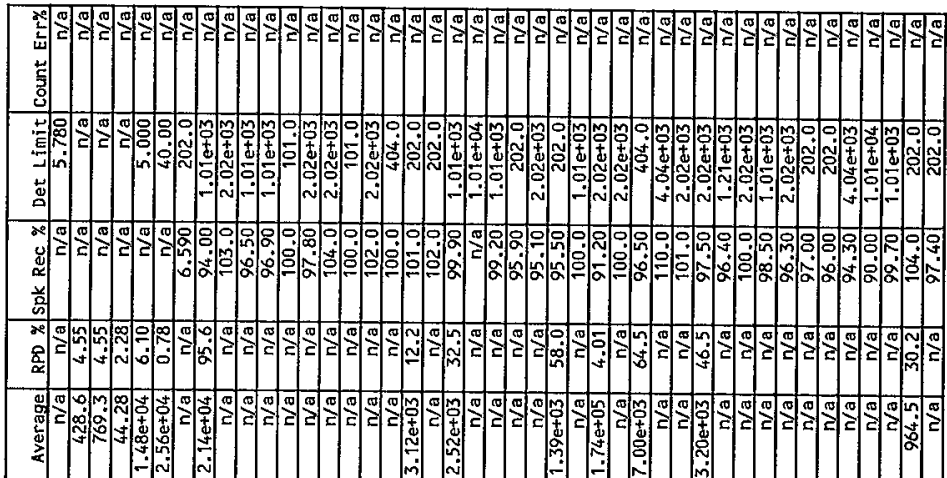

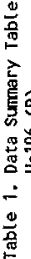

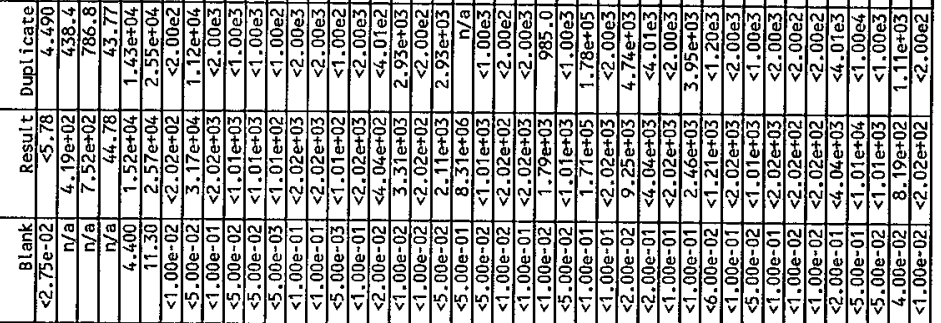

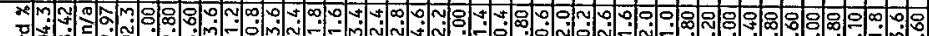
의 200 : 密

중

9 ㅇ․

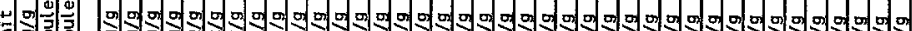

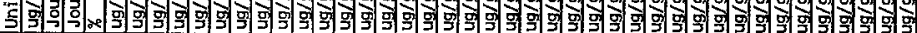

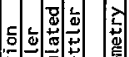

管

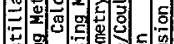

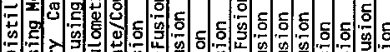

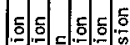

당

을 든

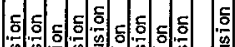

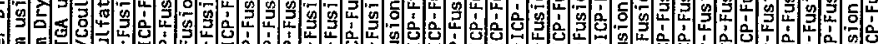

(

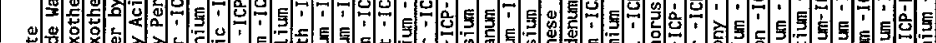

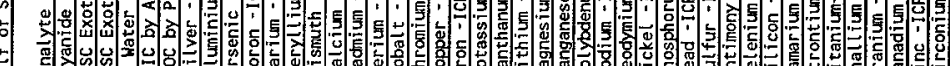

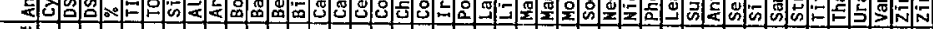
$\simeq$

Nov 
列

20-

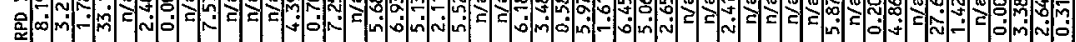

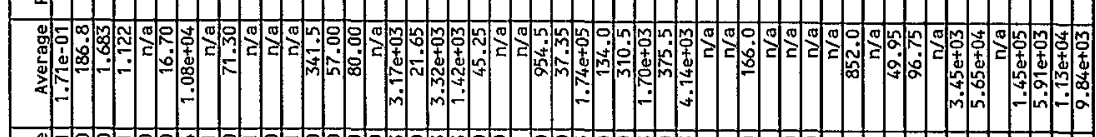

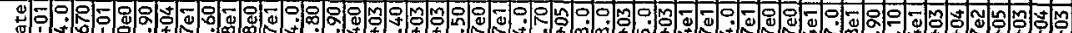

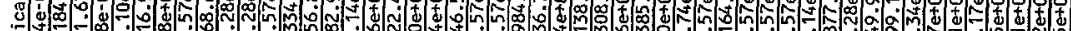

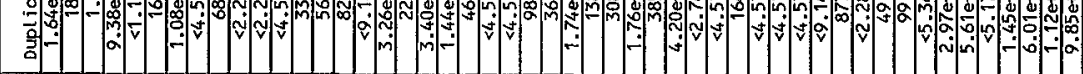

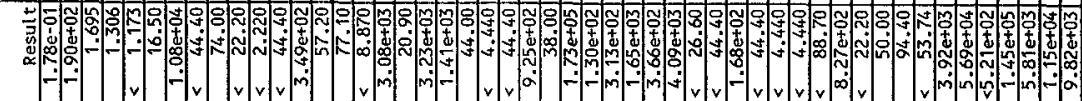

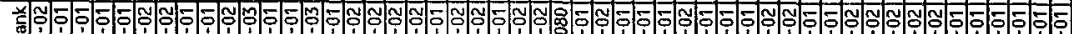

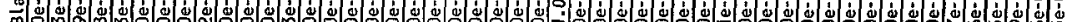

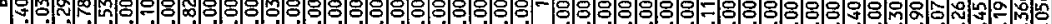

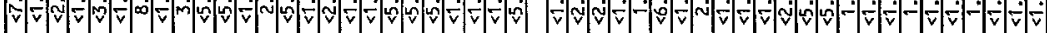
xकm

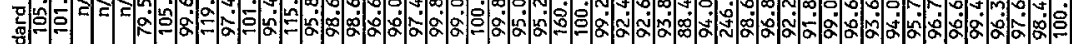
空

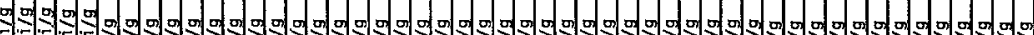

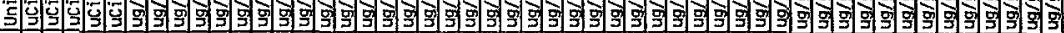

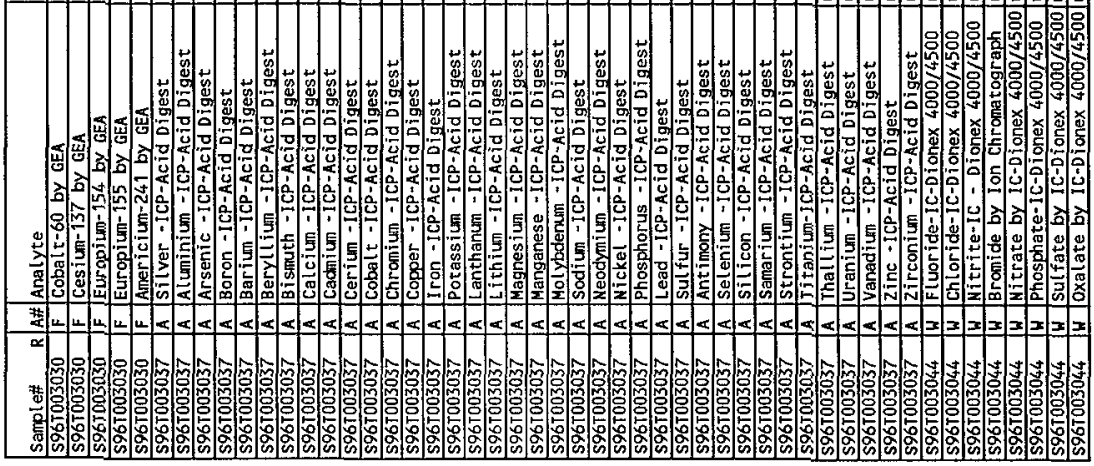
$\stackrel{2}{2}$ 
흔

영ำ?

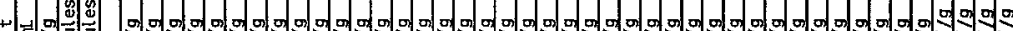

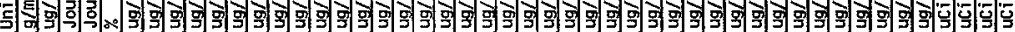

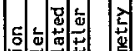

ข

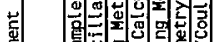

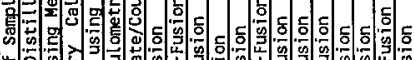

등듣ㅎㅇㄷㅇㅎㅎ

동

40

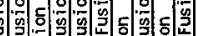

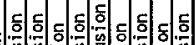

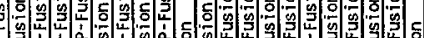

唯

$m M$

충

L 


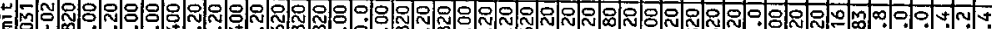
테 㟧

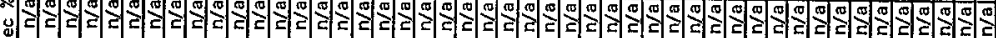
$*$

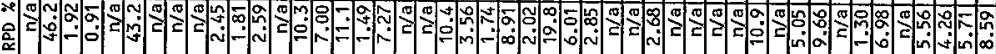

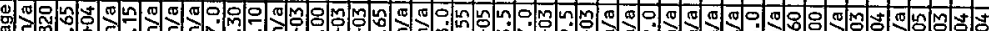

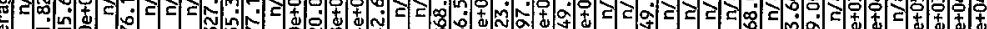

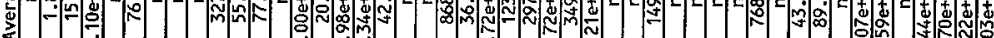

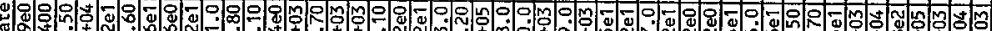

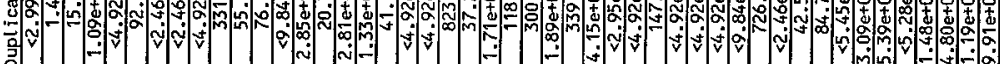

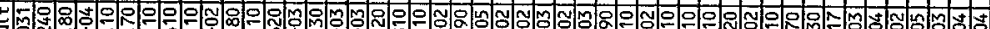

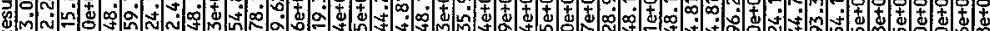

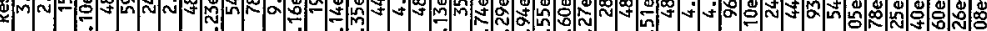

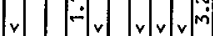

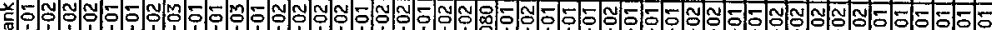
- n

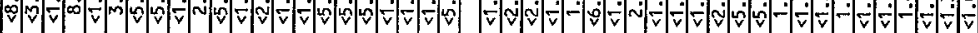

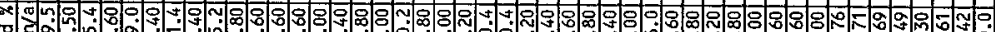
票 $\frac{9}{\mathrm{Q}}$

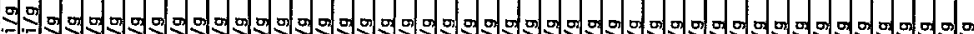

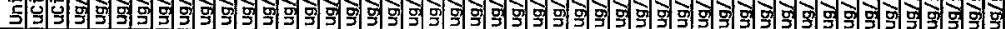

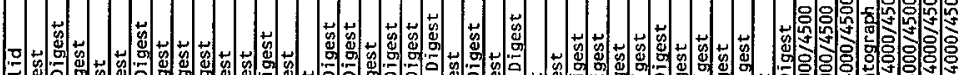

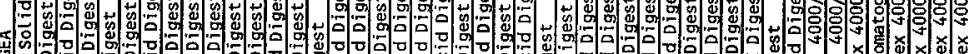
$\checkmark$ J

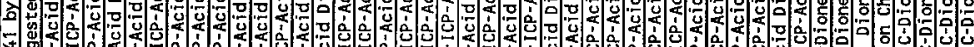

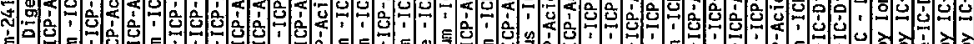

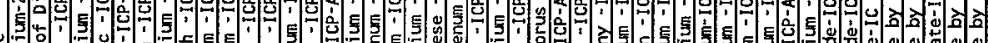

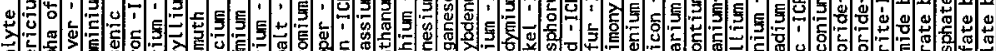

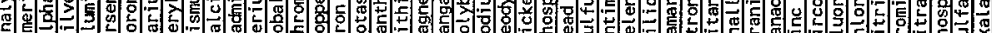

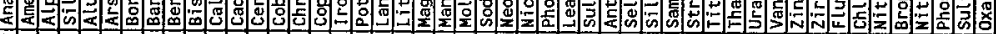

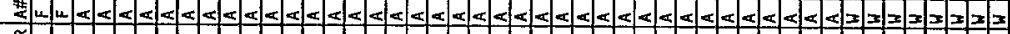

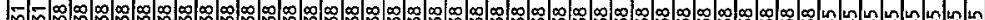

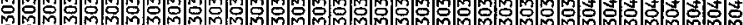

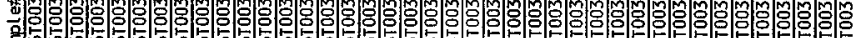
尊迢: 


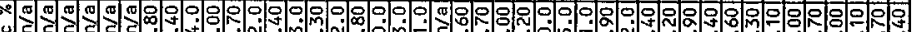

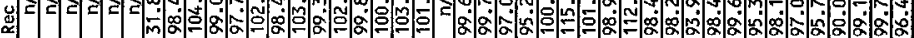
前

x-m.

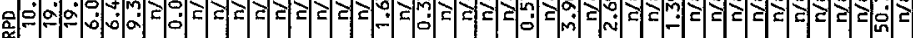

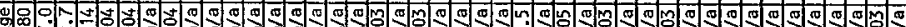

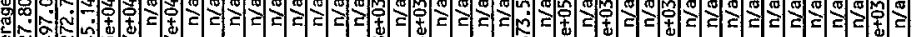

YOA

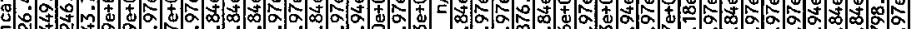
等

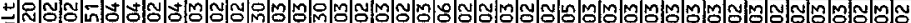

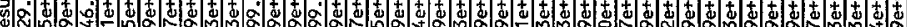
\%

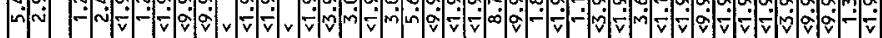

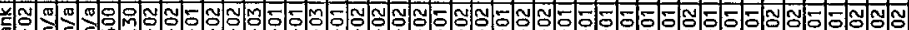

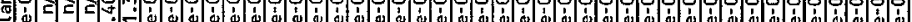

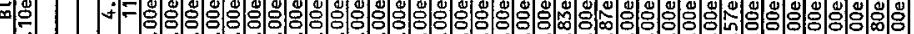

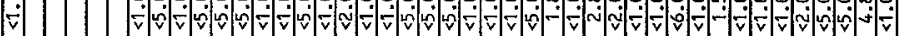

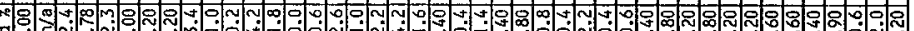
萢 홍 洁

aे

용ำ

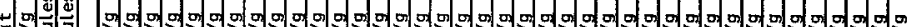

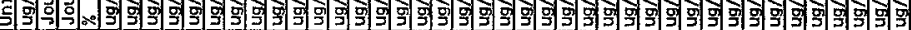

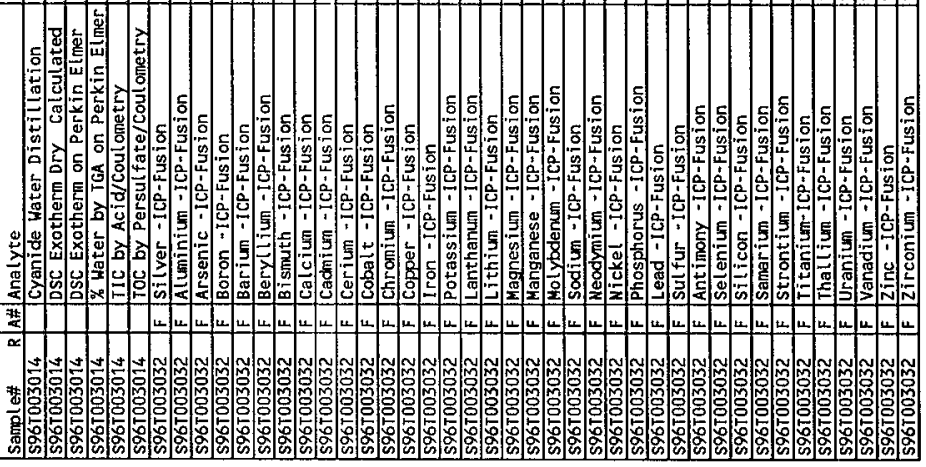



美 요요

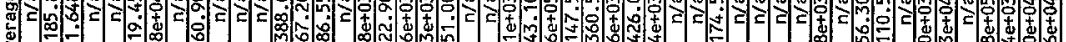
¿

(1)

ป

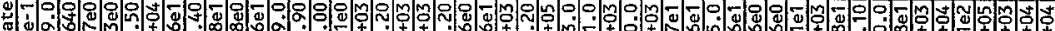

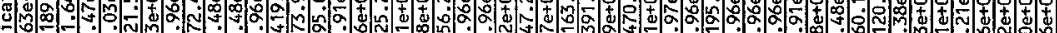

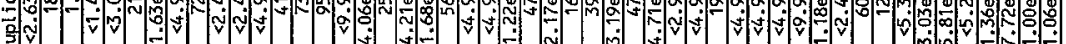
马े?

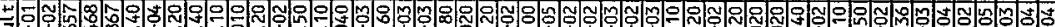

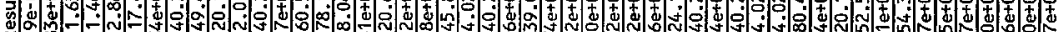

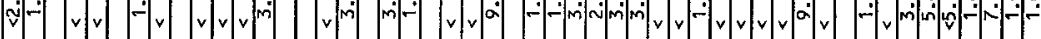
帝官

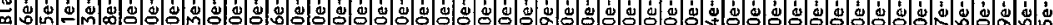

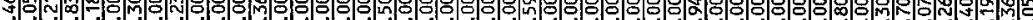

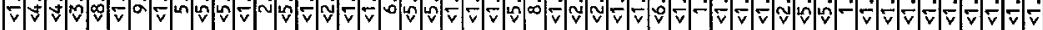
x n

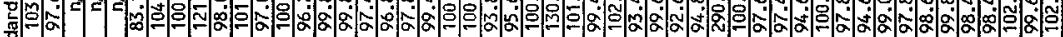
㟧

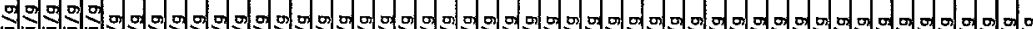

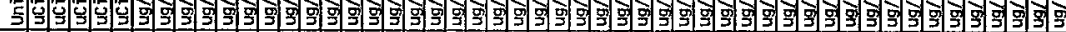

Y

g.

$\stackrel{\circ}{\circ}$

$\stackrel{\circ}{\circ}$

के

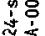

$+4$ (4) 怨

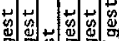

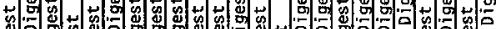

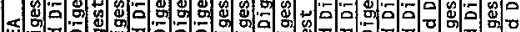

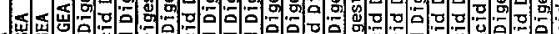

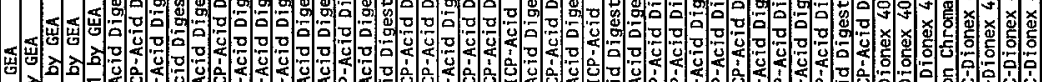

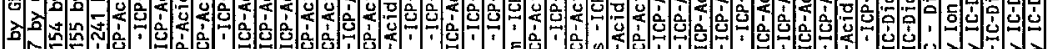
过

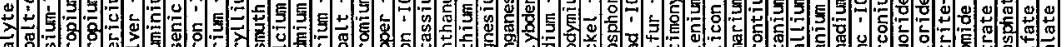

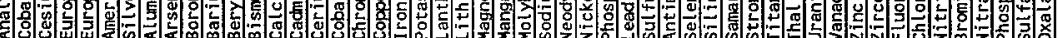

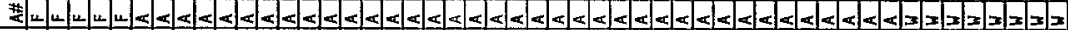
$\propto$ a a a

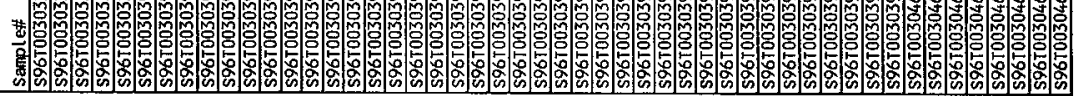




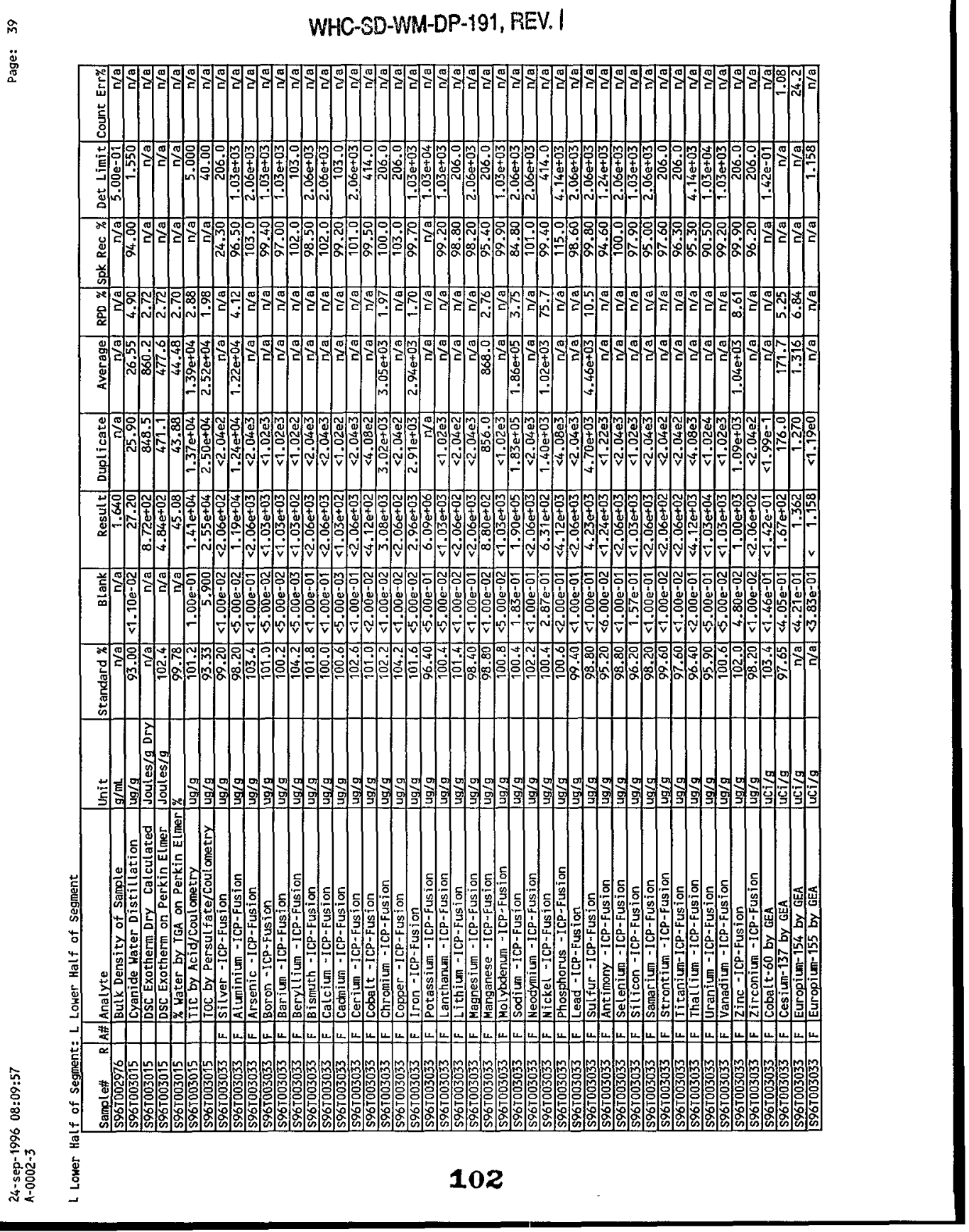




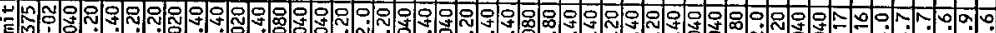
En 岁

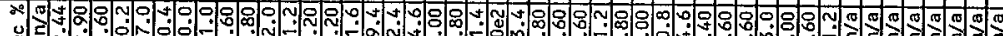

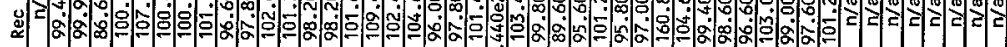
总 a :00

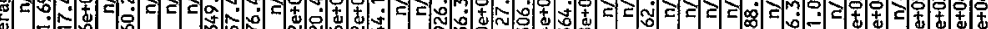

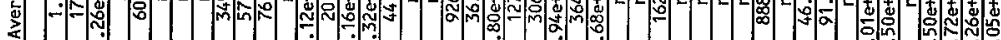

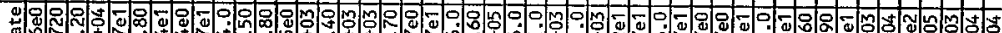

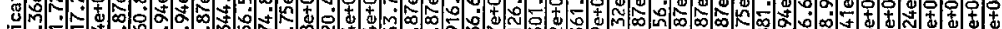
热

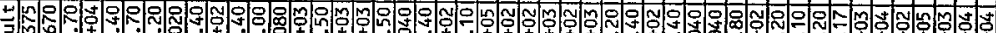

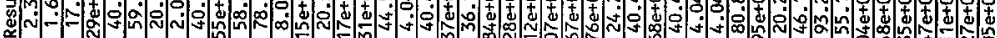
-

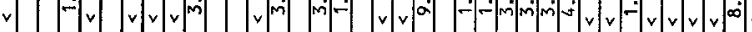

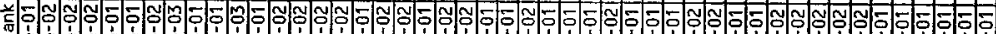

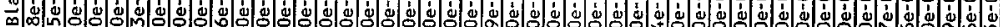
-

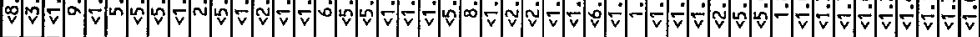

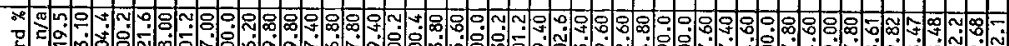
勿 屁

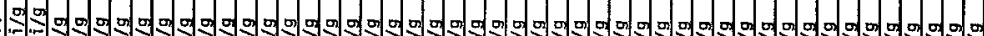
들

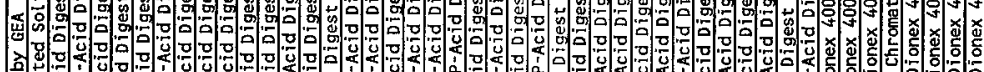
-

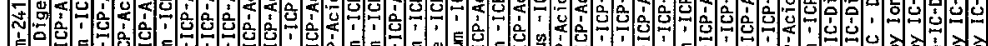

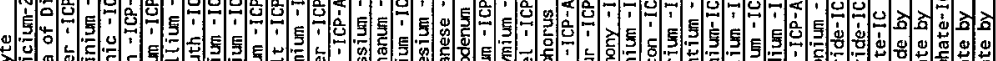

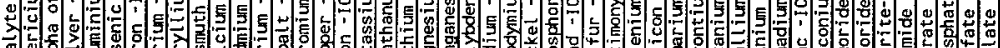

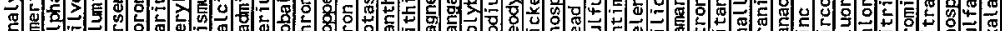

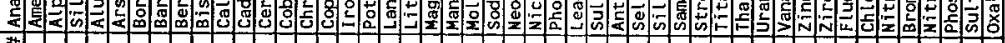

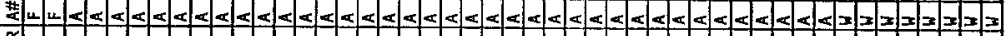
$\propto$ \%

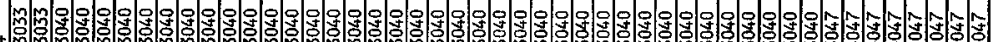

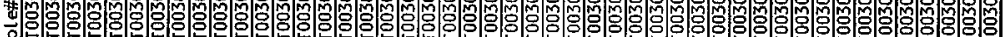


60

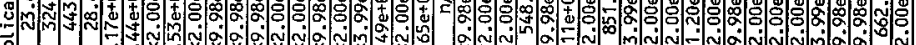

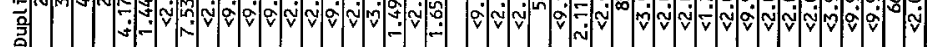

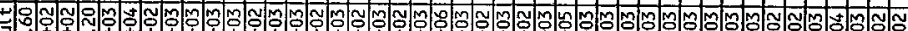

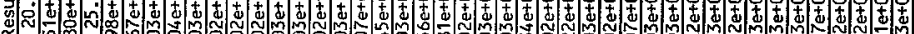
ฯ

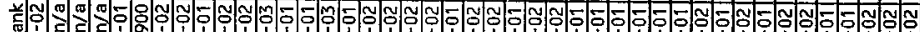

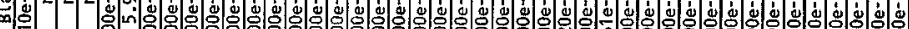

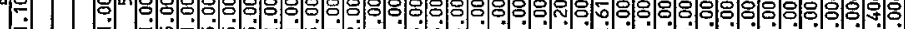

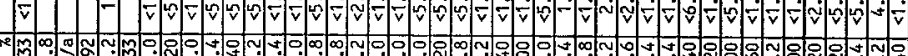
象 郆 究

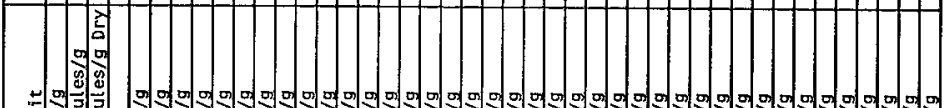

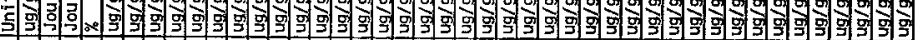

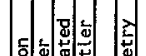

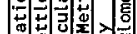

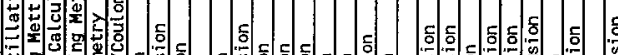

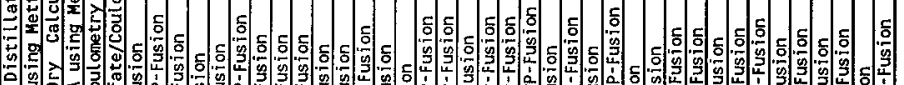
蒙 等

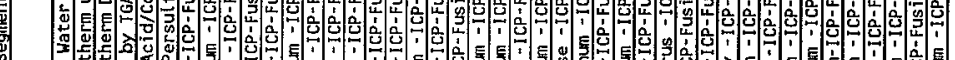
象:

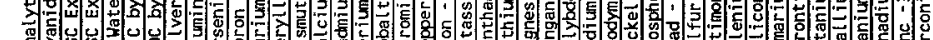

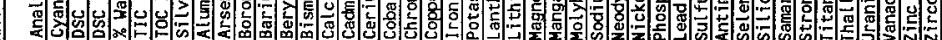

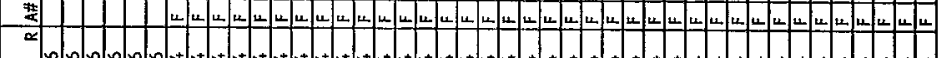
*

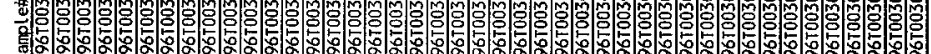

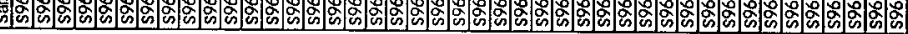




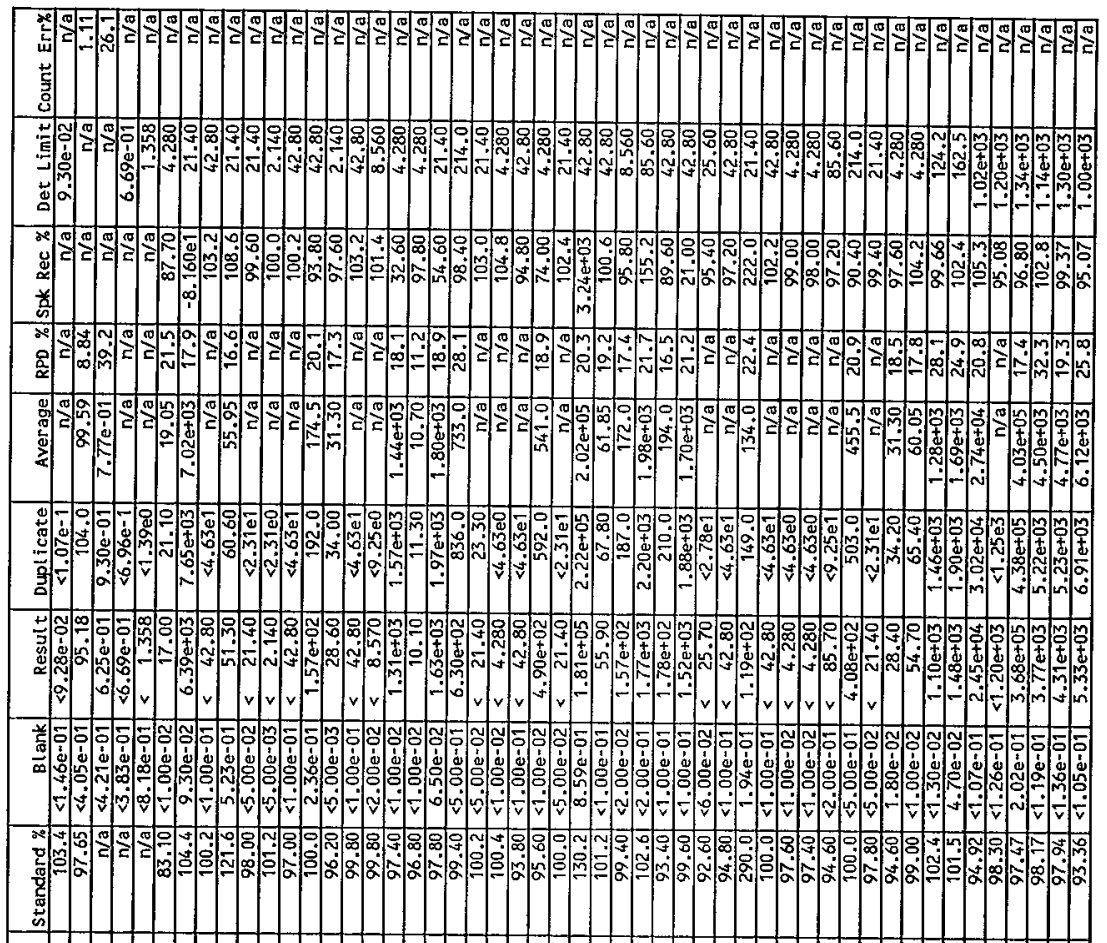

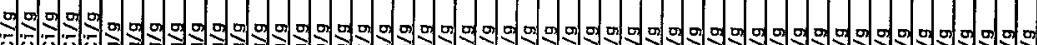

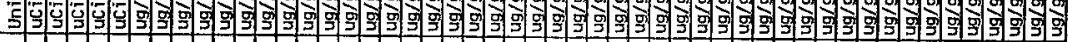

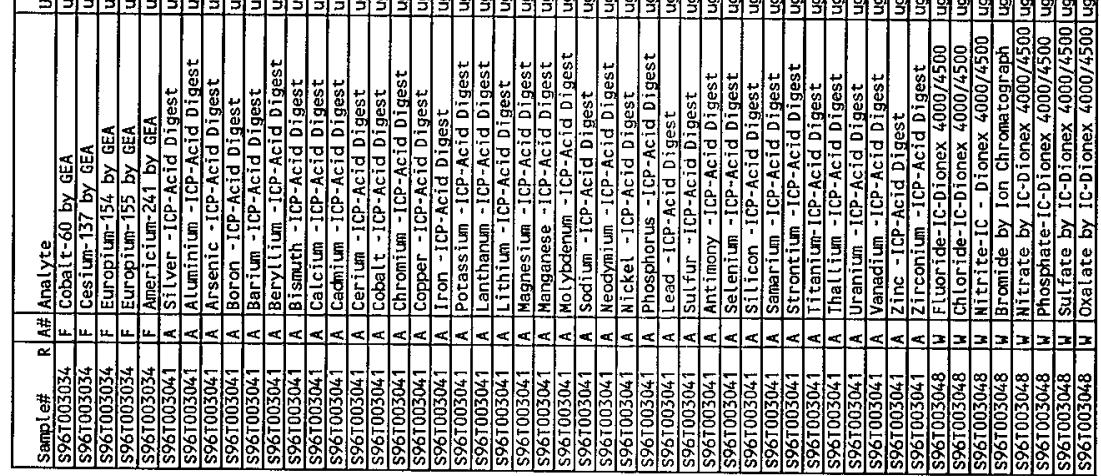
$\sigma_{m}$ 
0.0

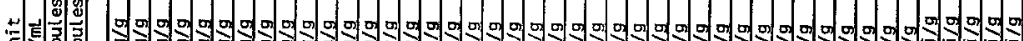

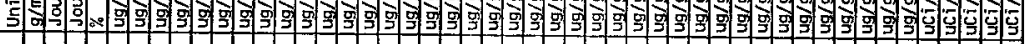

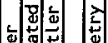

(1)

苍

क्व

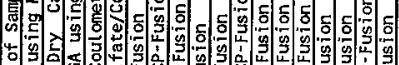

등ㄷㅇㄷㄷㄷㄷㅇㅢ

등 호

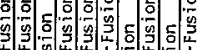

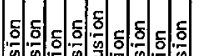

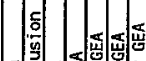

它

索4

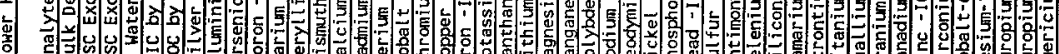

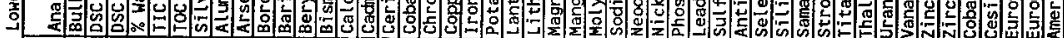

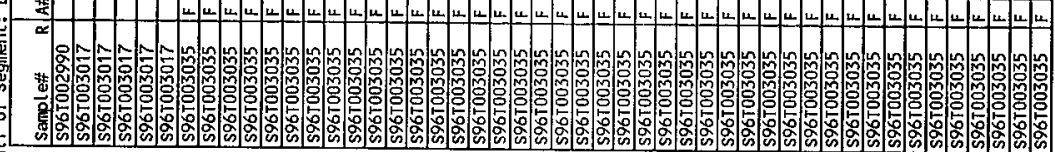




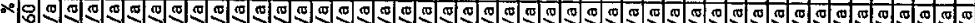

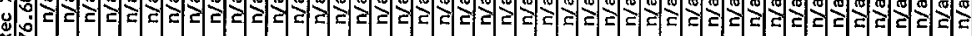
前

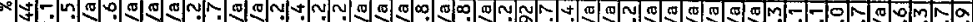

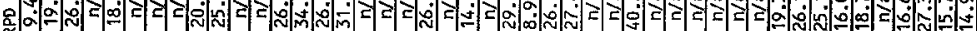

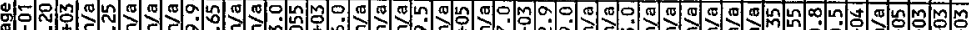

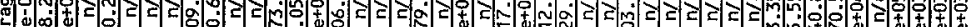
药

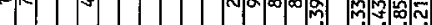

*马ी

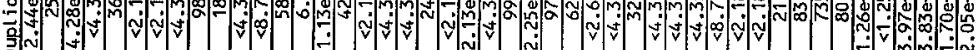

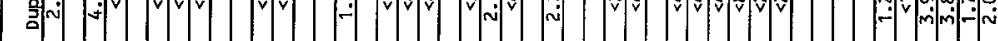

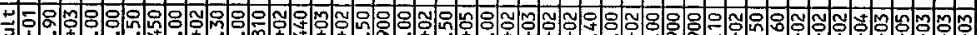

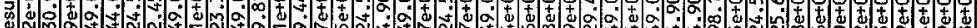
$+00200+40$

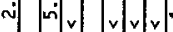

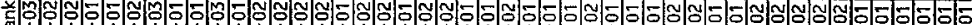
๑ on

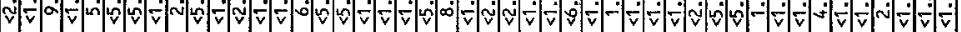
xny

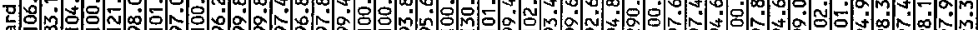

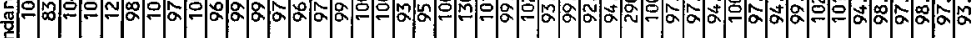
急

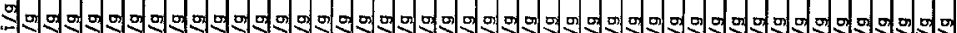

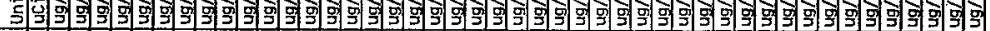

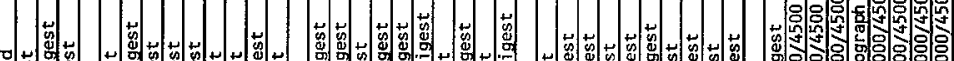

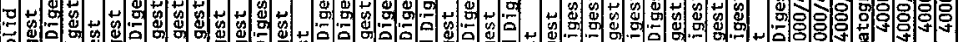

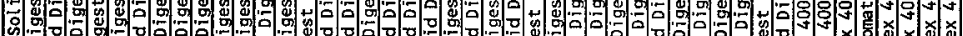

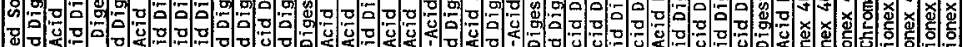

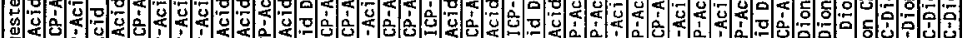

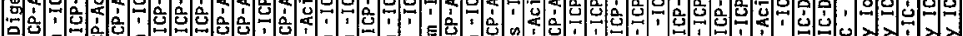

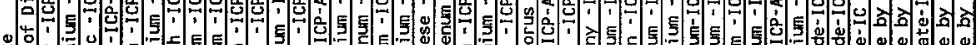

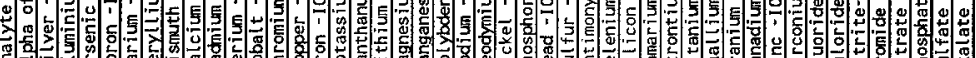

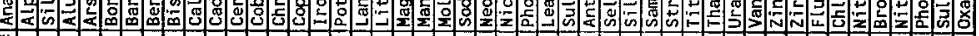

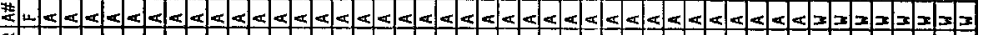
作 ث: 


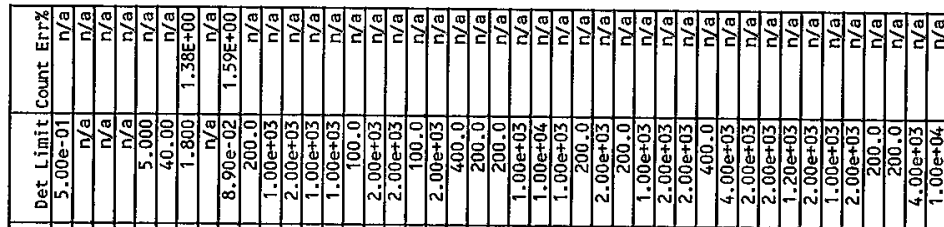

要

* ब.

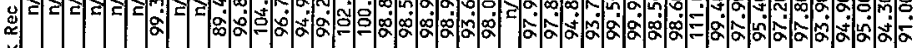
慈 *a

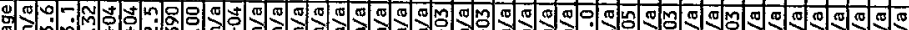

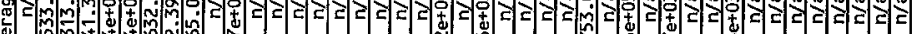

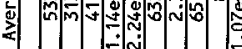

ॠ

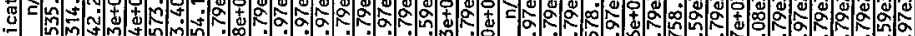

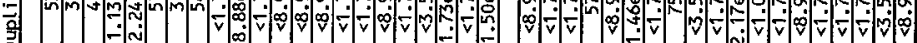

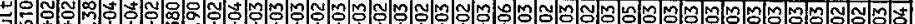

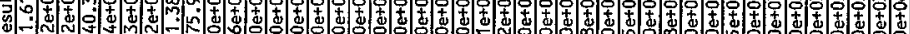
\%

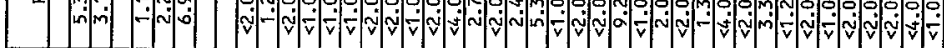

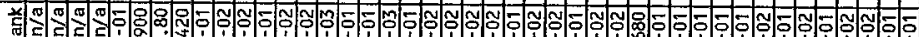

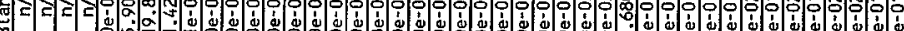

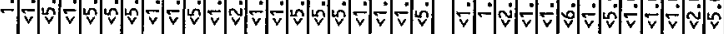

西

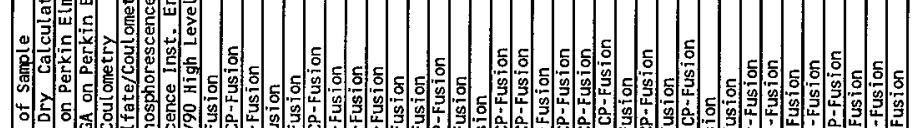

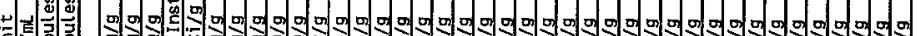

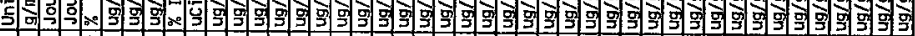

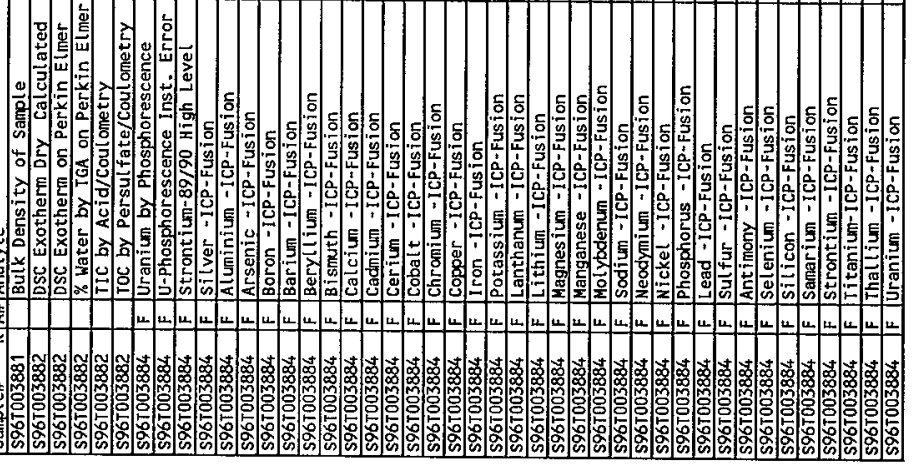




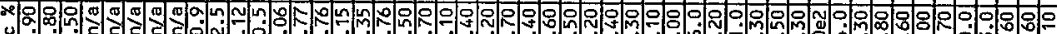

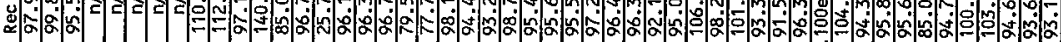
总

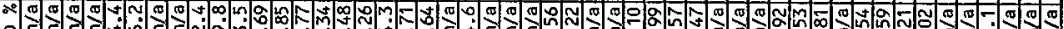

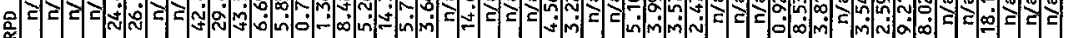

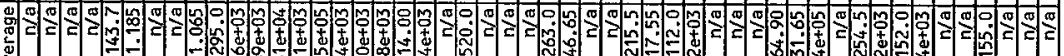

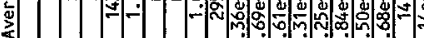

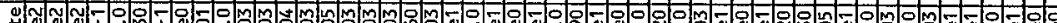

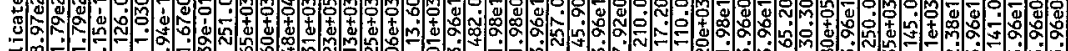

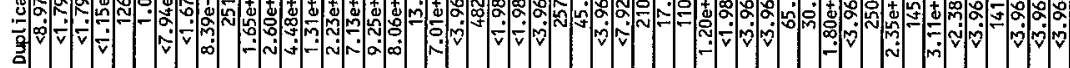

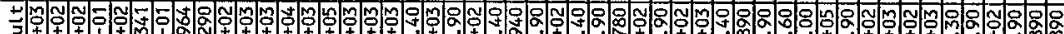

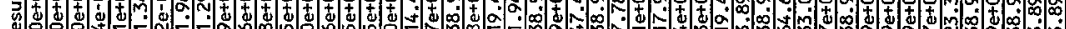

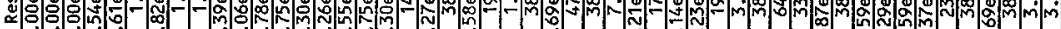

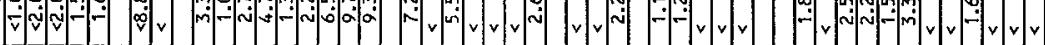

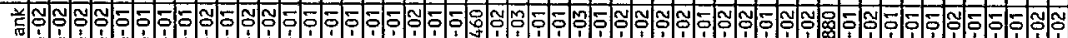
क ×

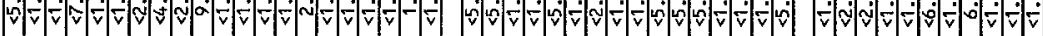

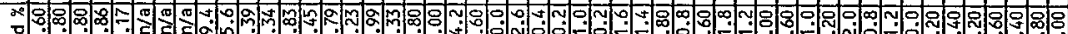

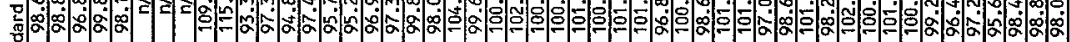
空

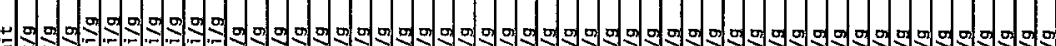

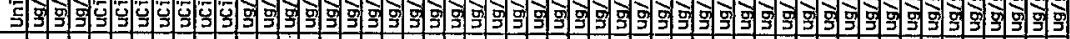

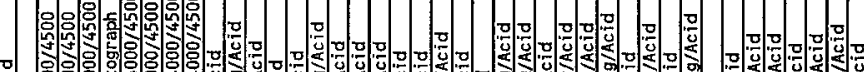
5

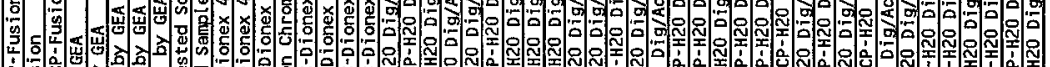
은

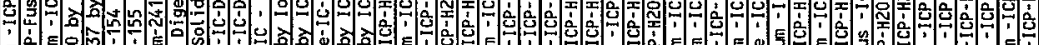

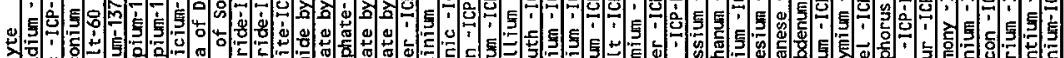

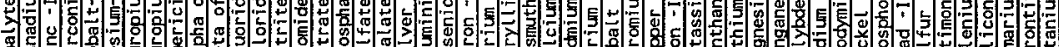

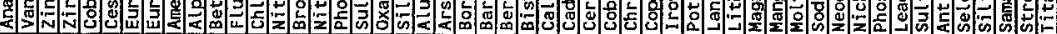

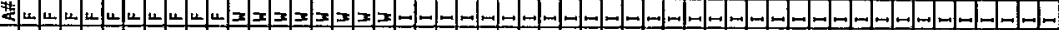
a $100-10$

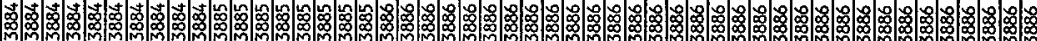

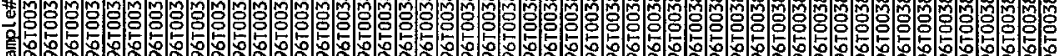

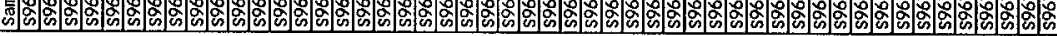




\section{WHC-SD-WM-DP-191, REV.1}

$\stackrel{\sim}{*}$

羿

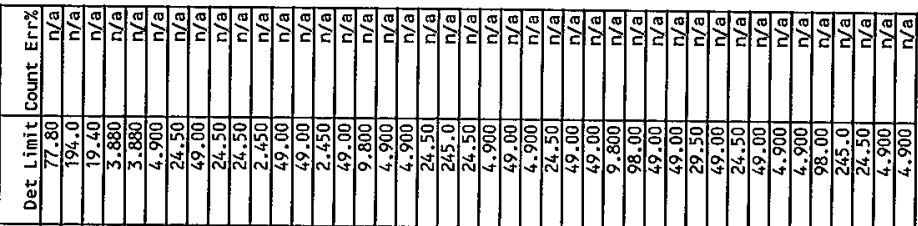

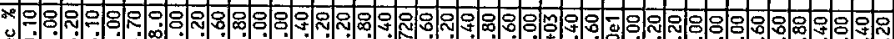

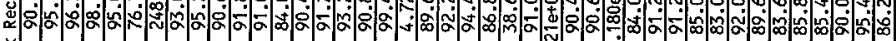
泀

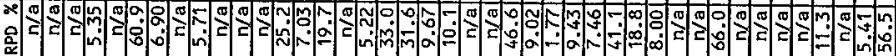
a ब0

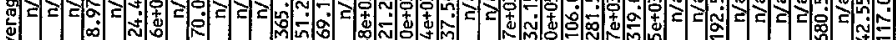

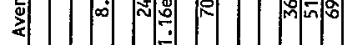

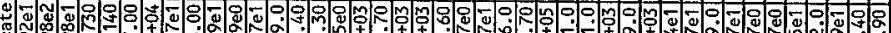

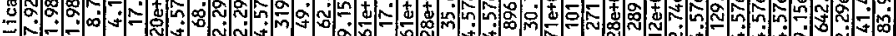

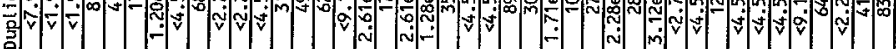

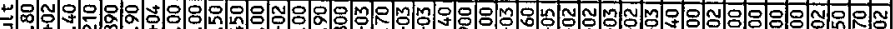

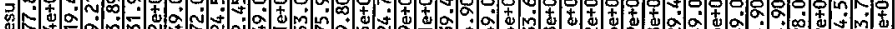

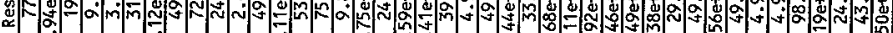
$\checkmark \div$

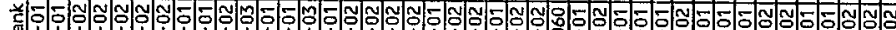

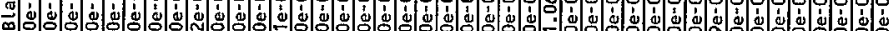
-

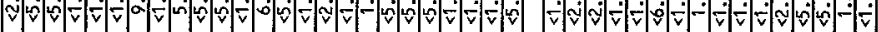

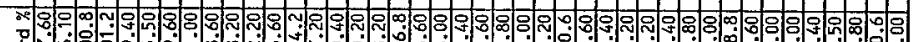

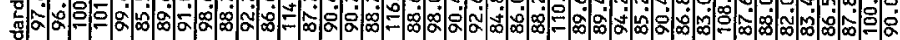
空

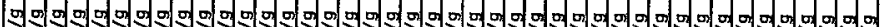

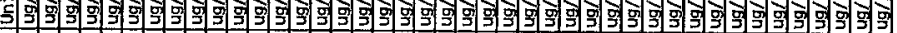

过

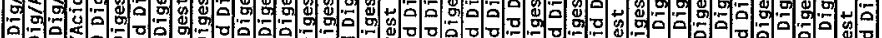

-

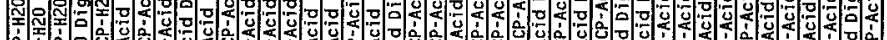

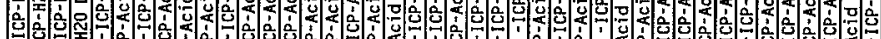

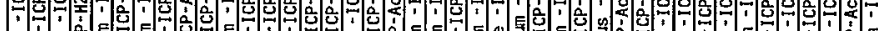

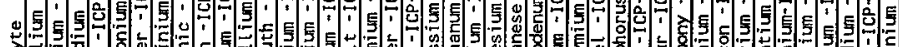

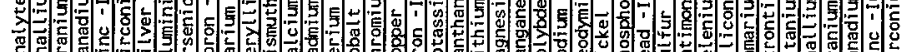

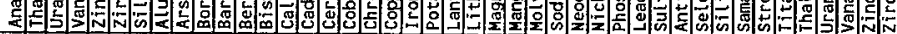

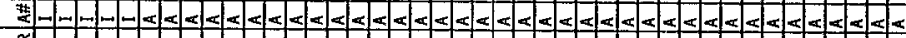

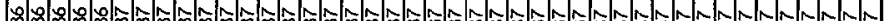

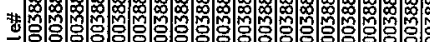

a

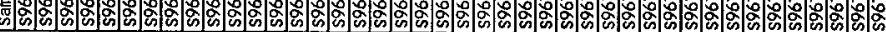


WHC-SD-WM-DP-191, REV. 1

PHOTOGRAPHS 
WHC-SD-WM-DP-191, REV. 1

THIS PAGE WAS INTENTIONALLY LEFT BLANK 


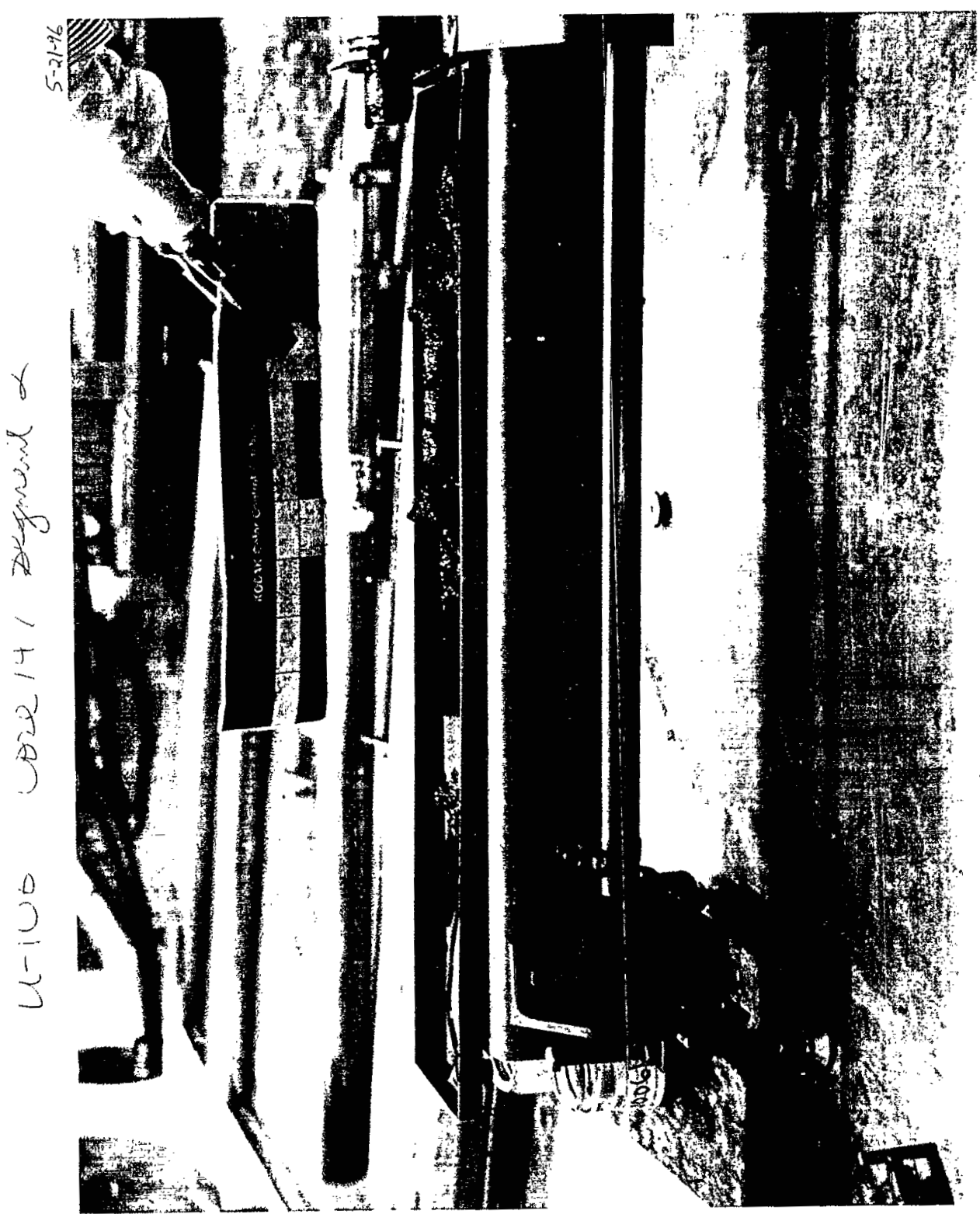

114 BEST AVAILABLE COPY 
WHC-SD-WM-DP-191, REV.I

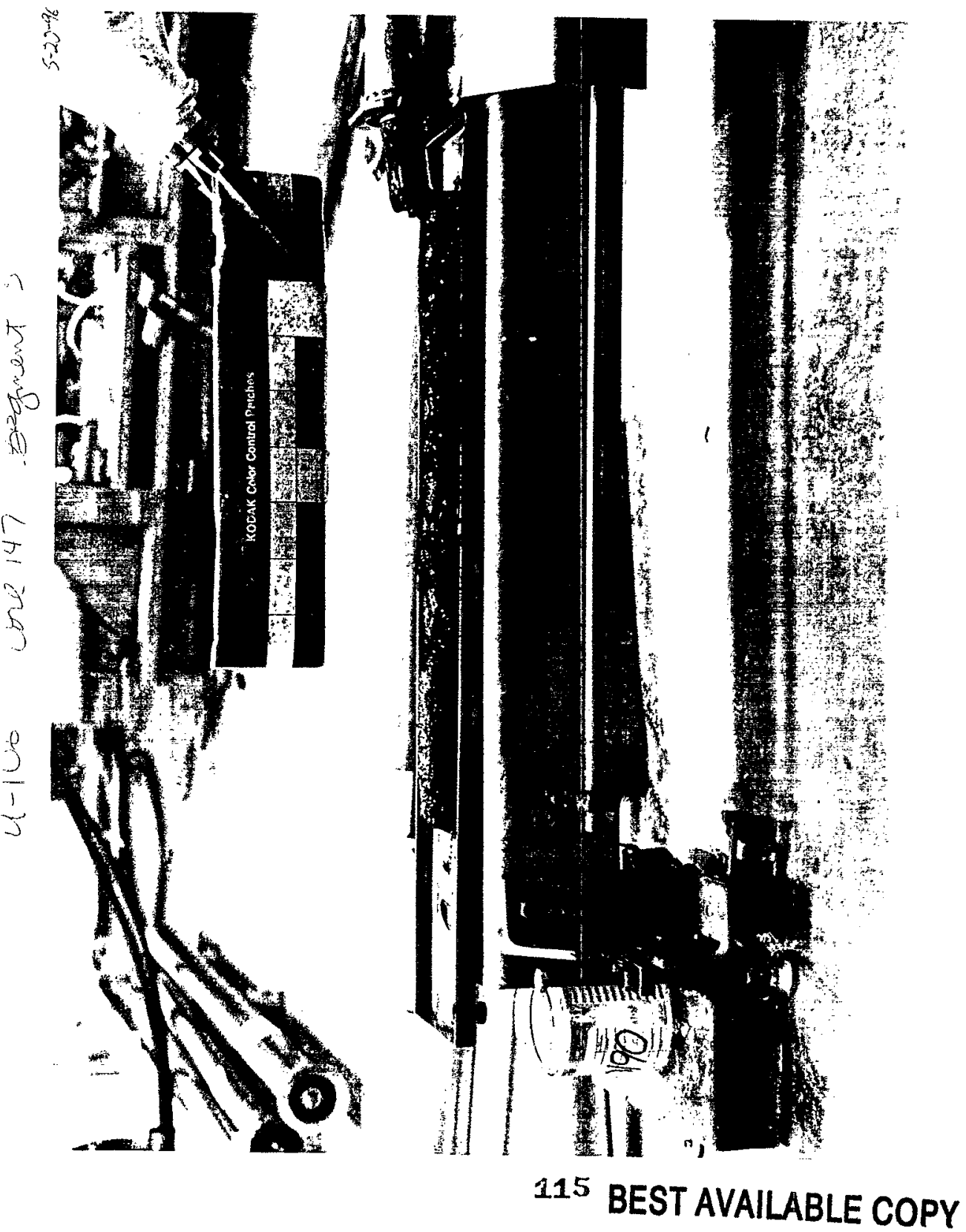


WHC-SD-WM-DP-19f, REV.1
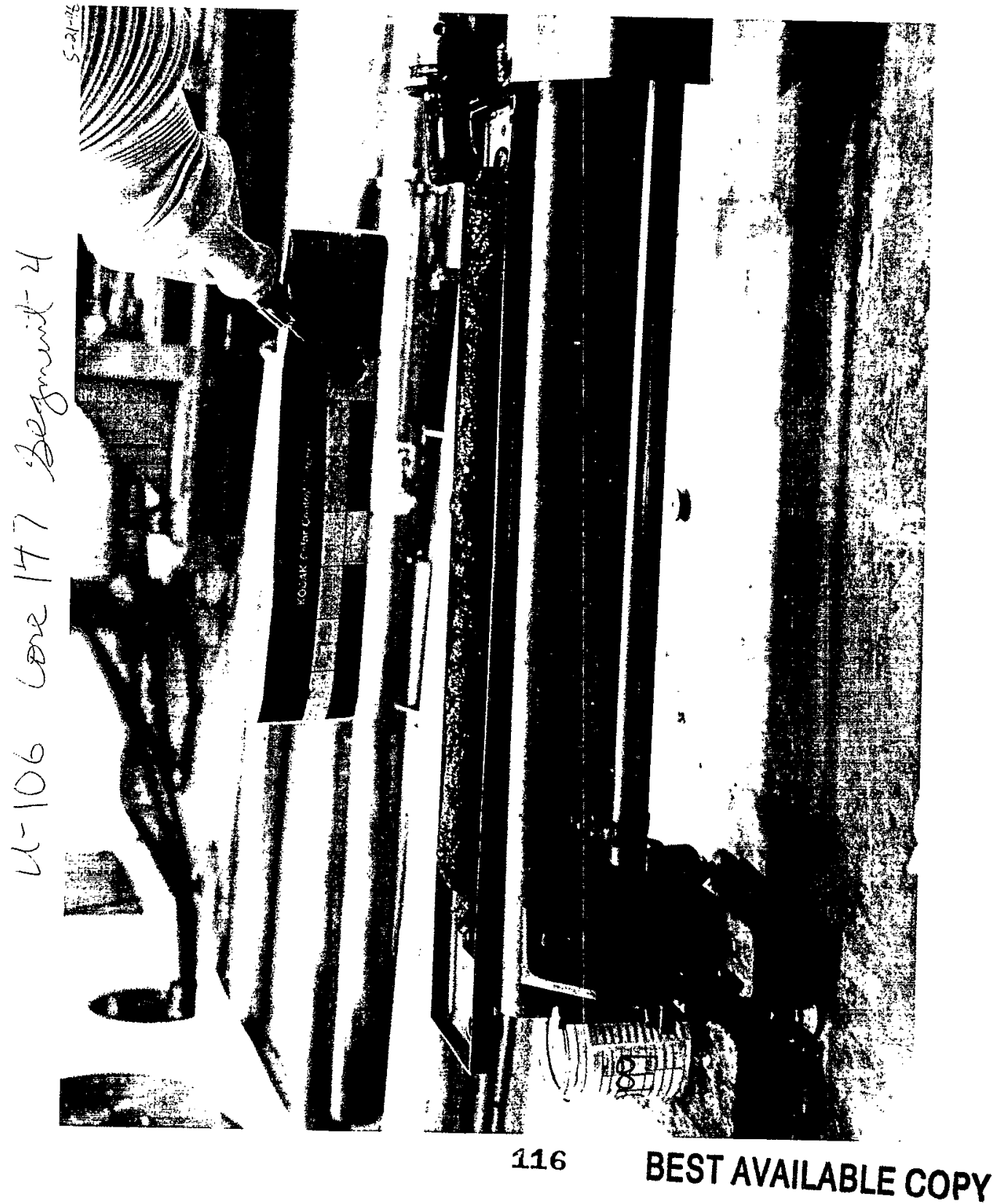
WHC-SD-WM-DP-191, REV.I

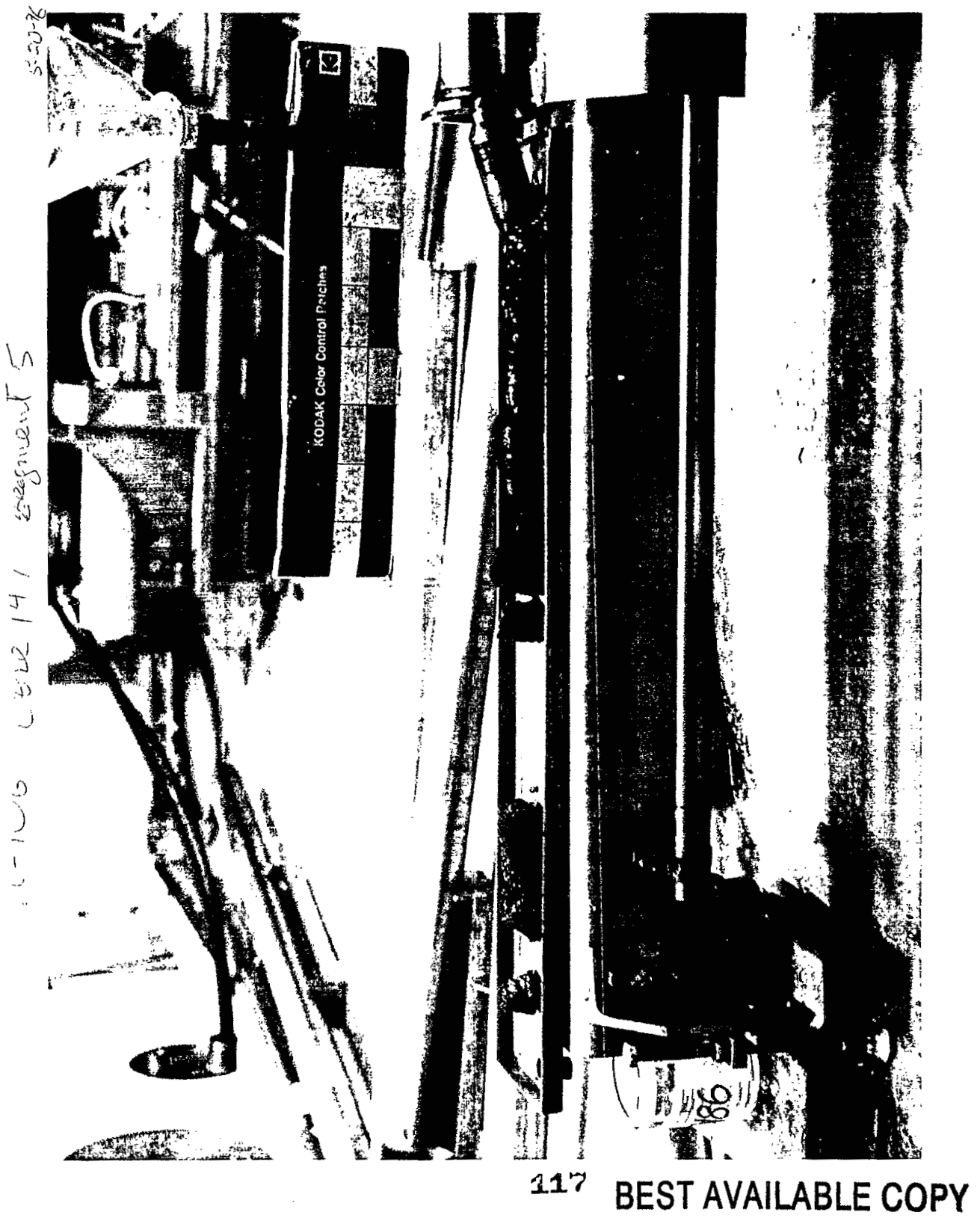




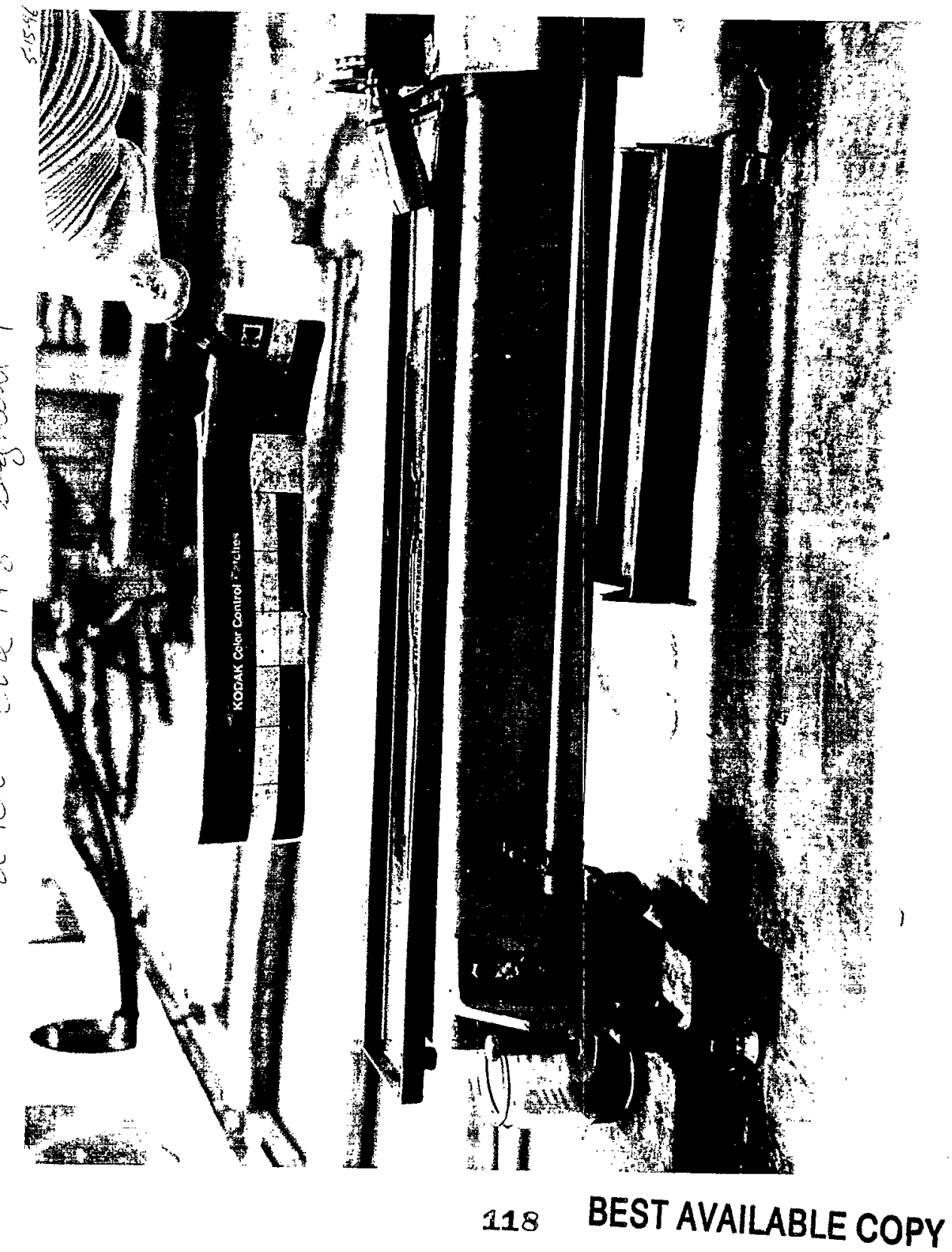


WHC-SD-WM-DP-191, REV.I

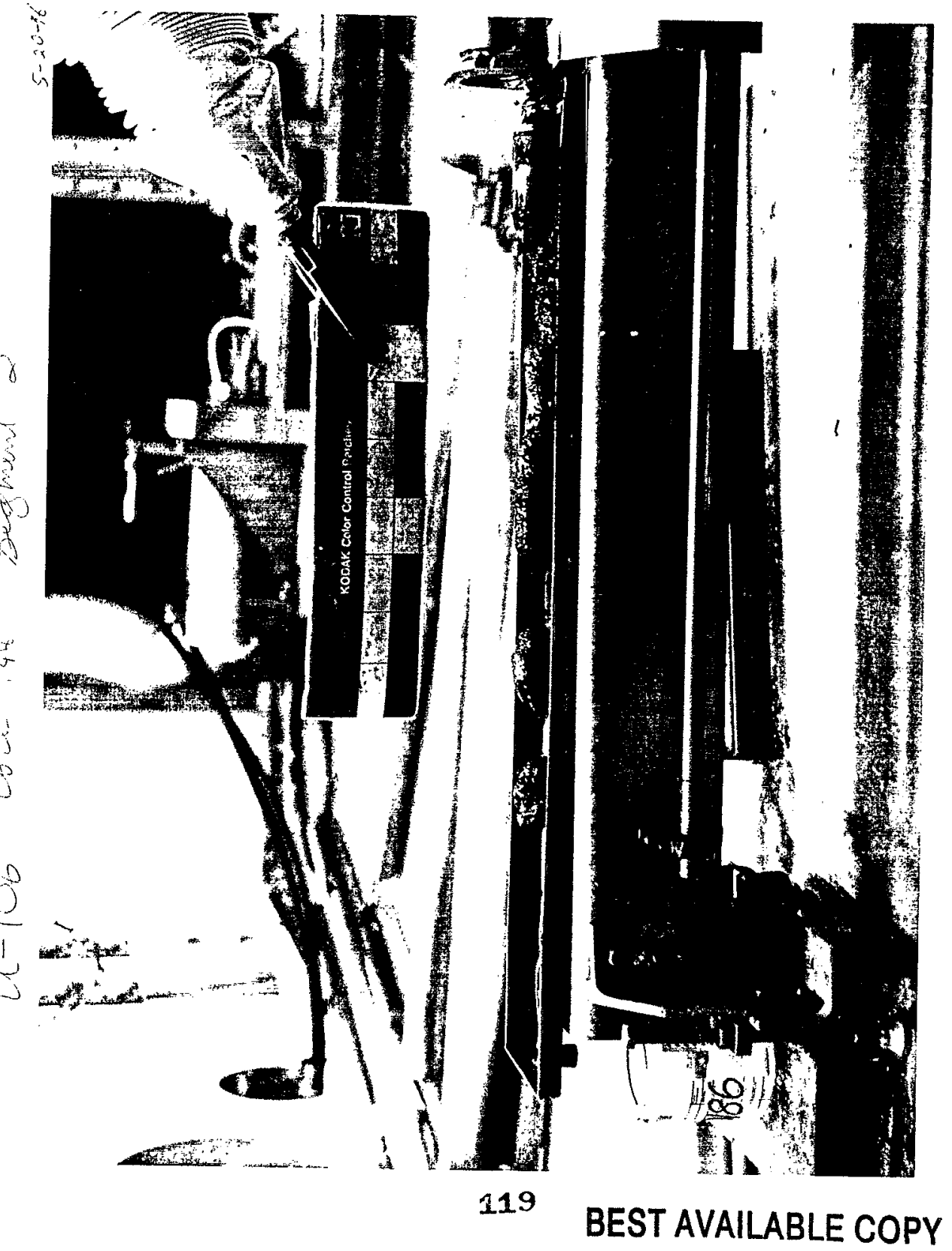




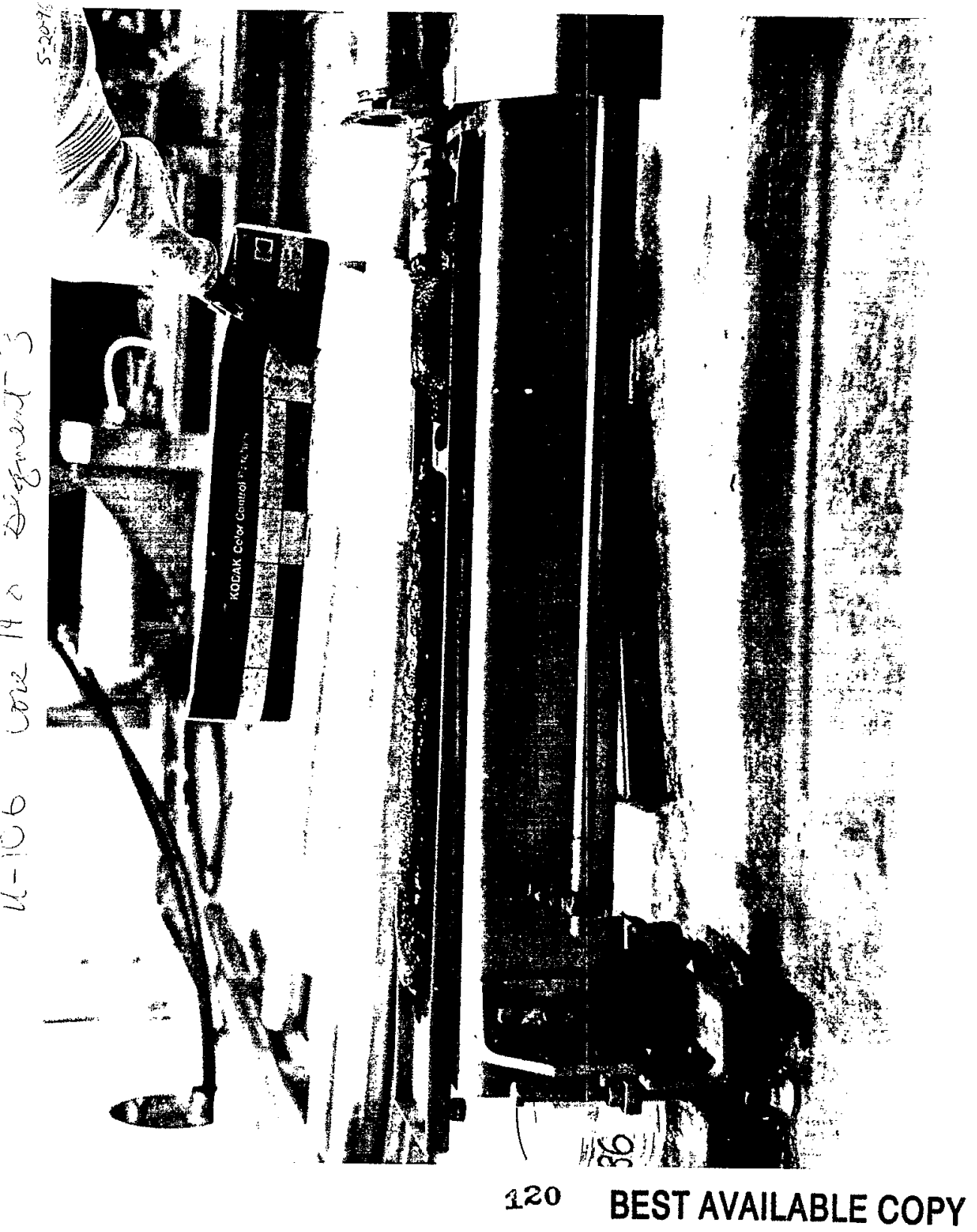


WHC-SD-WM-DP-191, REV.I

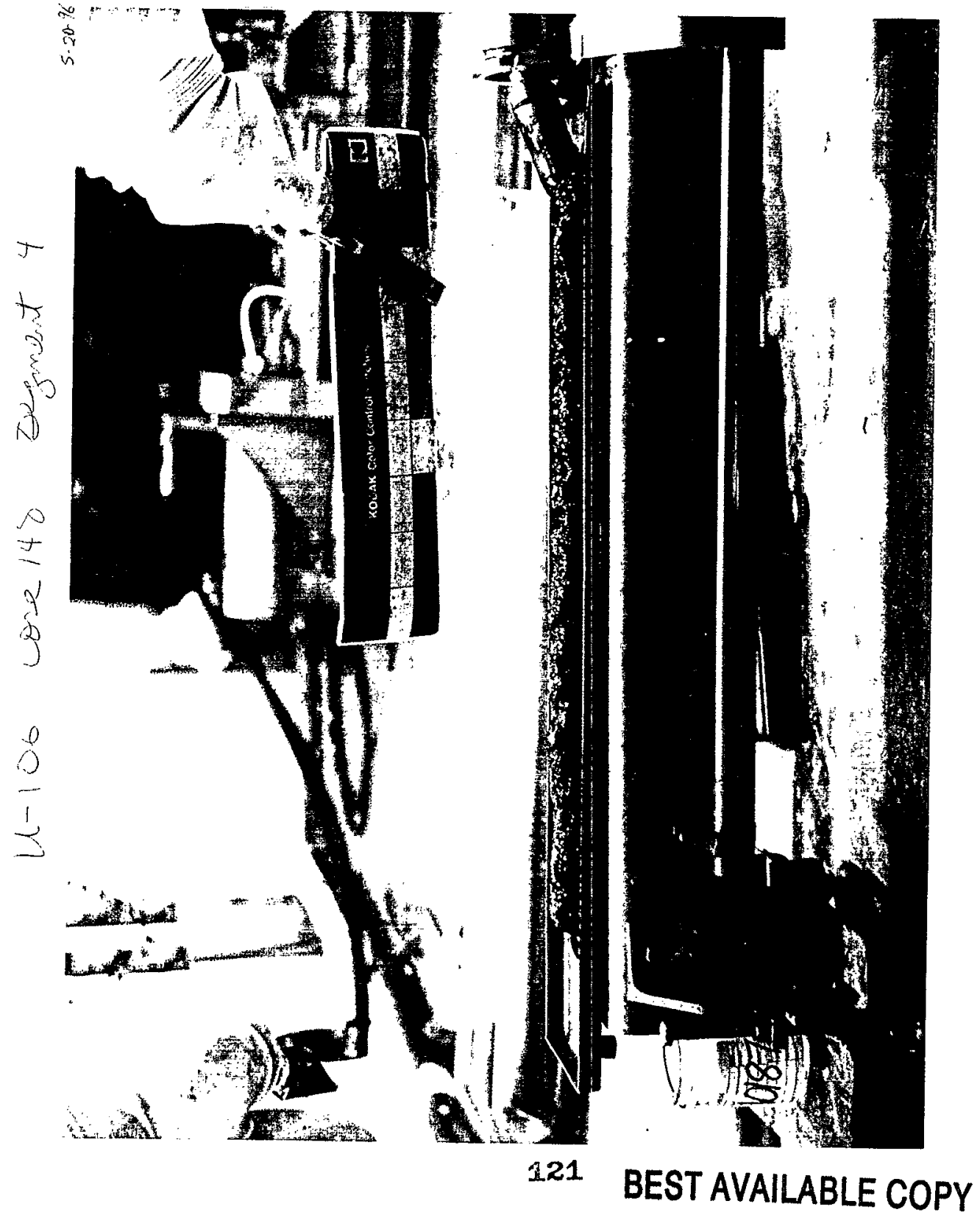




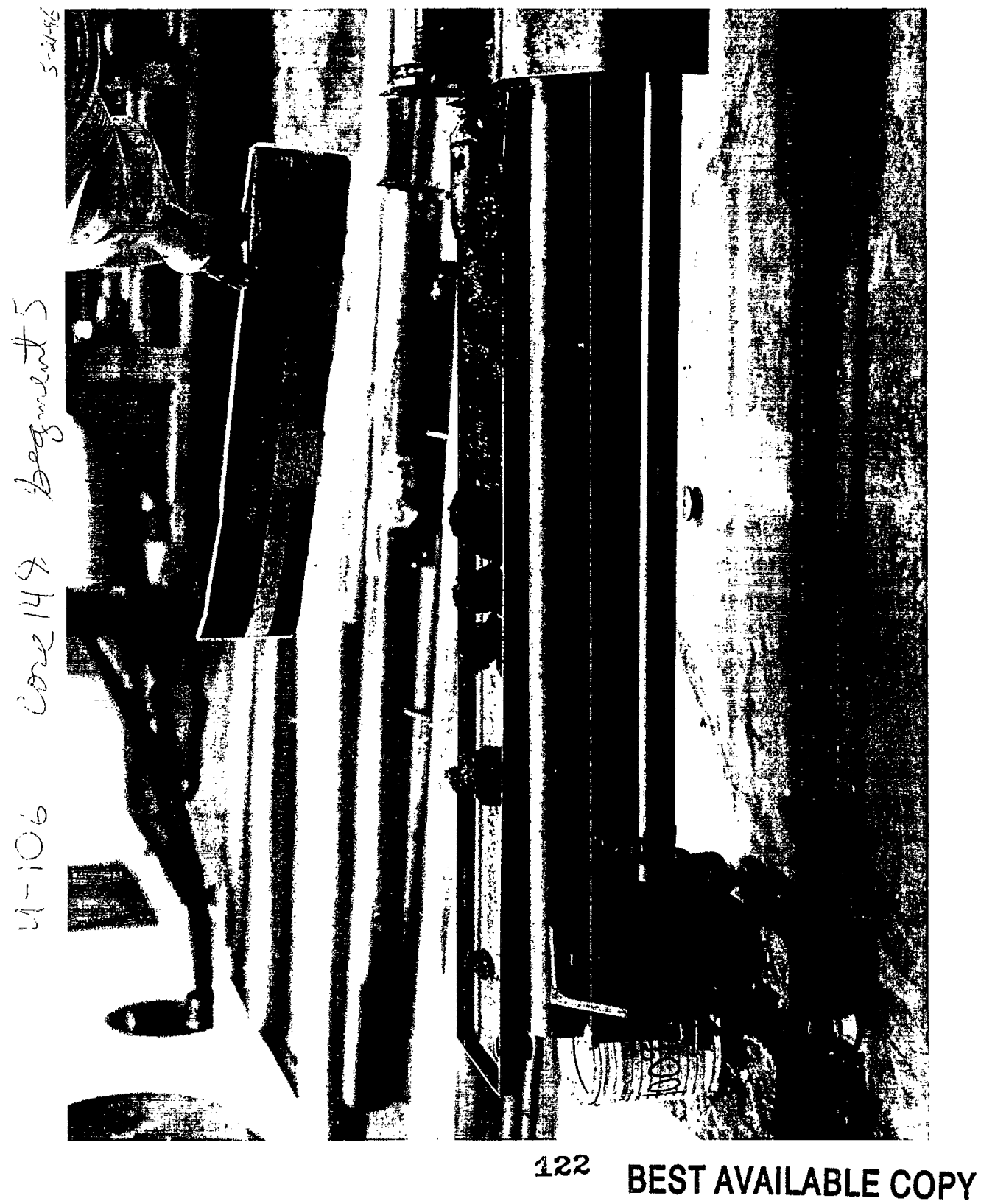


WHC-SD-HM-DP-191, REV. 1

U-106 EXTRUSION REPORTS 
WHC-SD-WM-DP-191, REV. 1

THIS PAGE WAS INTENTIONALLY LEFT BLANK

124 


\section{WHC-SD-WM-DP-191, REV.I}

\section{U-106 Core 147 Segment \#1 Riser \#19}

$\begin{array}{ll}\text { Date: } & \text { May 21, } 1996 \\ \text { Sampler serial \#: } & 95-099 S \\ \text { Sample \#: } & 96-215 \\ \text { Cask serial \#: } & \text { C-2001 } \\ \text { Logbook \#: } & \text { WHC-N-1342 } \\ \text { Labcore \#: } & \text { S96T002857 }\end{array}$

Notes:

* Hot Cell Temp 80.2 degrees F. Humidity $28 \%$.

* Check weights: $20 \mathrm{gms}=20.00$ grams $500 \mathrm{gms}=500.01$ grams

* Dose rate through the drill string was $1.4 \mathrm{R} / \mathrm{hr}$.

* Liner liquid: None observed.

* Sampler valve closed before and after cutting cables.

* Extruded sample and performed photography.

Sample information:

* Collected approximately $230 \mathrm{mLs}$ of drainable liquid (L1). The liquid was amber in color and clear. No solids were present other than those present in drainable liquid. Subsampled liquid in one jar.

Subsample information:

* Subsampled Liquids: Drainable Liquid

U-106 Core 147 Segment \#1 Biser 19:

-Jar \# 10090 (250 mL jar size)

-314.5 gram net weight

Notes:

* A total of 314.5 grams of solids were collected. The dose rate through the drill string was $1.4 \mathrm{R} / \mathrm{hr}$. 


\section{WHC-SD-WM-DP-191, REV.I}

\section{U-106 Core 147 Segment \#2 Riser \#19}

Date:

May 21, 1996

Sampler serial \#:

94-318S

Sample \#:

Cask serial \#: 70

Logbook \#:

96-216

Labcore \#:

WHC-N-1342

S96T002858

Notes:

* Hot Cell Temp 80.1 degrees F. Humidity $31 \%$.

* Check weights: $20 \mathrm{gms}=20.00$ grams $500 \mathrm{gms}=500.01$ grams

* Dose rate through the drill string was $1.4 \mathrm{R} / \mathrm{hr}$.

* Liner liquid: None observed.

* Sampler valve closed before and after cutting cables.

* Extruded sample and performed photography.

Sample information:

* Collected approximately $90 \mathrm{mLs}$ of drainable liquid (L1). The liquid was dark brown in color and opaque. Extruded approximately 8 inches of solids. The solids were dark brown in color and the texture resembled a sludge slurry (D2). The solids were subsampled in one jar.

Subsample information:

* Subsampled Liquids: Drainable Liquid

U-106 Core 147 Segment \#2 Riser 19:

-Jar \# 10061 (125 mL jar size)

-132.0 gram net weight

* Subsampled Solids: Upper Half Solids

U-106 Core 147 Segment \#2 Riser 12 : -Jar \# 10201 (250 mL jar size)

-201.2 gram net weight

Notes:

* A total of 333.2 grams of liquid and solids were collected. The dose rate through the drill string was $1.4 \mathrm{R} / \mathrm{hr}$. 


\section{WHC-SD-WM-DP-191, REV.|}

\section{U-106 Core 147 Segment \#3 Riser \#19}

Date:

May 20, 1996

Sampler serial \#:

Sample \#:

95-112S

Cask serial \#: 73

Logbook \#:

96-217

Labcore \#:

WHC-N-1342

S96T002811

Notes:

* $\quad$ Hot Cell Temp 79.9 degrees F. Humidity $28 \%$.

* Check weights: $20 \mathrm{gms}=20.00$ grams $500 \mathrm{gms}=500.02$ grams

* Dose rate through the drill string was $1.5 \mathrm{R} / \mathrm{hr}$.

* Liner liquid: None observed.

* Sampler valve closed before and after cutting cables.

* Extruded sample and performed photography.

Sample information:

* No drainable liquid. Extruded approximately 17 inches of solids. The solids were dark brown in color and the texture resembled a sludge slurry (D2). The solids were subsampled in half segments.

Subsample information:

* Subsampled Solids: Upper Half Solids

U-106 Core 147 Segment \#3 Riser 19: -Jar \# 10190 (250 mL jar size)

-178.7 gram net weight

* Subsampled Solids: Lower Half Solids

U-106 Core 147 Segment \#3 Riser 19:

-Jar \# 10191 (250 mL jar size)

-200.8 gram net weight

Notes:

* A total of 379.5 grams of solids were collected. The dose rate through the drill string was $1.5 \mathrm{R} / \mathrm{hr}$. 


\section{U-106 Core 147 Segment \#4 Riser \#19}

Date: $\quad$ May 21, 1996

Sampler serial \#: $\quad$ 95-313S

Sample \#: $\quad 96-218$

Cask serial \#: C-1021

Logbook \#: WHC-N-1342

Labcore \#: $\quad$ S96T002812

Notes:

* Hot Cell Temp 80.1 degrees F. Humidity $31 \%$.

* $\quad$ Check weights: 20 gms $=20.00$ grams 500 gms $=500.02$ grams

* Dose rate through the drill string was $1.4 \mathrm{R} / \mathrm{hr}$.

* Liner liquid: None observed.

* Sampler valve closed before and after cutting cables.

* Extruded sample and performed photography.

Sample information:

* No drainable liquid. Extruded approximately 19 inches of solids. The solids were dark brown in color and the texture resembled a sludge slurry (D2). The solids were subsampled in half segments.

Subsample information:

* Subsampled Solids: Upper Half Solids

U-106 Core 147 Segment \# 4 Riser 19:

-Jar \# 10194 (250 mL jar size)

-211.3 gram net weight

* Subsampled Solids: Lower Half Solids

U-106 Core 147 Segment \# 4 Riser 19:

-Jar \# 10199 (250 mL jar size)

-213.8 gram net weight

Notes:

* A total of 425.1 grams of solids were collected. The dose rate through the drill string was $1.4 \mathrm{R} / \mathrm{hr}$. 


\section{WHC-SD-WM-DP-191, REV.I}

\section{U-106 Core 147 Segment \#5 Riser \#19}

Date: $\quad$ May 20, 1996

Sampler serial \#: $\quad 94-052 \mathrm{~S}$

Sample \#: $\quad$ 96-219

Cask serial \#: 1011-C

Logbook \#: $\quad$ WHC-N-1342

Labcore \#: $\quad$ S96T002813

Notes:

* Hot Cell Temp 79.5 degrees F. Humidity $28 \%$.

* Check weights: 20 gms $=20.00$ grams 500 gms $=500.02$ grams

* Dose rate through the drill string was $700 \mathrm{mR} / \mathrm{hr}$.

* Liner liquid: None observed.

* Sampler valve closed before and after cutting cables.

* Extruded sample and performed photography.

Sample information:

* No drainable liquid. Extruded approximately 14 inches of solids. The lower portion of solids were 4 inches in length, dark brown in color and the texture resembled a wet salt (M3). The upper portion was 10 inches in length, dark brown in color and the texture resembled a sludge slurry (D2). The solids were subsampled in half segments.

Subsample information:

* Subsampled Solids: Upper Half Solids U-106 Core 147 Segment \# 5 Riser 19: -Jar \# 10184 (250 mL jar size)

-260.1 gram net weight

* Subsampled Solids: Lower Half Solids U-106 Core 147 Seqment \# 5 Riser 19: -Jar \# 10069 (125 mL jar size)

-91.5 gram net weight

Notes:

* A total of 351.6 grams of solids were collected. The dose rate through the drill string was $700 \mathrm{mR} / \mathrm{hr}$. 


\section{WHC-SD-WM-DP-191, REV.I}

\section{U-106 Core 148 Segment \#1 Riser \#2}

Date: May 15, 1996

Sampler serial \#: $\quad 95-062 S$

Sample \#: $\quad 96-220$

Cask serial \#: 1010-C

Logbook \#: WHC-N-1177

Labcore \#: $\quad$ S96T002823

Notes:

* $\quad$ Hot Cell Temp 78.8 degrees F. Humidity $43 \%$.

* Check weights: $20 \mathrm{gms}=20.00$ grams $500 \mathrm{gms}=500.01$ grams

* Dose rate through the drill string was $1 \mathrm{R} / \mathrm{hr}$.

* Liner liquid: None observed.

* Sampler valve closed before and after cutting cables.

* Extruded sample and performed photography.

Sample information:

* Collected approximately $80 \mathrm{mLs}$ of drainable liquid (L1). The liquid was amber in color and opaque. No solids were present. The liquid was subsampled in one jar.

Subsample information:

* Subsampled Liquid: Drainable Liquid

U-106 Core 148 Segment \# 1 Riser 2;

-Jar \# 10185 (250 mL jar size)

-116.5 gram net weight

Notes:

* A total of 116.5 grams of liquid were collected. The dose rate through the drill string was $1 \mathrm{R} / \mathrm{hr}$. 
WHC-SD-WM-DP-191, REV.I

\section{U-106 Core 148 Segment \#2 Riser \#2}

Date: May 20, 1996

Sampler serial \#: $\quad 95-156 \mathrm{~S}$

Sample \#: $\quad 96-221$

Cask serial \#: C-1016

Logbook \#: WHC-N-1342

Labcore \#: $\quad$ S96T002824

Notes:

* $\quad$ Hot Cell Temp 79.8 degrees F. Humidity $28 \%$.

* Check weights: 20 gms $=20.00$ grams 500 gms $=500.02$ grams

* Dose rate through the drill string was $1.5 \mathrm{R} / \mathrm{hr}$.

* Liner liquid: None observed.

* Sampler valve closed before and after cutting cables.

* Extruded sample and performed photography.

Sample information:

* Collected approximately $80 \mathrm{mLs}$ of drainable liquid (L1). The liquid was brown gray in color and opaque. Extruded approximately 9 inches of solids. The solids were light gray to dark brown in color and the texture resembled a sludge slurry (D2).

Subsampled solids in half segments.

Subsample information:

* Subsampled Liquid: Drainable Liquid U-106 Core 148 Segment \#2 Riser 2: -Jar \# 10063 (125 mL jar size)

-124.1 gram net weight

* Subsampled Solids: Upper Half Solids U-106 Core 148 Segment \#2 Riser 2: -Jar \# 10053 (125 mL jar size)

-104.3 gram net weight

* Subsampled Solids: Lower Half Solids U-106 Core 148 Segment \# 2 Riser 2: -Jar \# 10058 (125 mL jar size)

-120.8 gram net weight 


\section{WHC-SD-WM-DP-191, REV.I}

Notes:

A total of 349.2 grams of liquid and solids were collected. The dose rate through the drill string was $1.5 \mathrm{R} / \mathrm{hr}$.

\section{U-106 Core 148 Segment \#3 Riser \#2}

Date: $\quad$ May 20, 1996

Sampler serial \#: $\quad 95-1345$

Sample \#: $\quad 96-222$

Cask serial \#: 61

Logbook \#:

Labcore \#:

WHC-N-1342

S96T002818

Notes:

* Hot Cell Temp 79.8 degrees F. Humidity $28 \%$.

* Check weights: 20 gms $=20.00$ grams 500 gms $=500.03$ grams

* Dose rate through the drill string was $1500 \mathrm{mR} / \mathrm{hr}$.

* Liner liquid: None observed.

* Sampler valve closed before and after cutting cables.

* Extruded sample and performed photography.

Sample information:

* No drainable liquid. Extruded approximately 18 inches of solids. The solids were dark brown in color and the texture resembled a wet sludge (D3). Subsampled solids in half segments.

Subsample information:

* $\quad$ Subsampled Solids: Upper Half Solids U-106 Core 148 Segment \# 3 Riser 2 :

-Jar \# 10180 (250 mL jar size)

-202.8 gram net weight

* Subsampled Solids: Lower Half Solids

U-106 Core 148 Segment \#3 Riser 2 :

-Jar \# 10186 (250 mL jar size)

-197.4 gram net weight

Notes: 


WHC-SD-WM-DP-191, REV.I

* A total of 400.2 grams of solids were collected. The dose rate through the drill string was $1500 \mathrm{mR} / \mathrm{hr}$. 
WHC-SD-WM-DP-191, REV.I

\section{U-106 Core 148 Segment \#4 Riser \#2}

Date: $\quad$ May 20, 1996

Sampler serial \#: $\quad 95-064 \mathrm{~S}$

Sample \#: $\quad 96-223$

Cask serial \#: C-2008

Logbook \#: $\quad$ WHC-N-1342

Labcore \#: $\quad$ S96T002819

Notes:

* Hot Cell Temp 79.6 degrees F. Humidity $28 \%$.

* Check weights: $20 \mathrm{gms}=20.00 \mathrm{grams} 500 \mathrm{gms}=500.03 \mathrm{grams}$

* Dose rate through the drill string was $1.3 \mathrm{R} / \mathrm{hr}$.

* Liner liquid: None observed.

* Sampler valve closed before and after cutting cables.

* Extruded sample and performed photography.

Sample information:

* No drainable liquid. Extruded approximately 19 inches of solids. The solids were dark brown in color and the texture resembled a wet sludge (D3). Subsampled solids in half segments.

Subsample information:

* Subsampled Solids: Upper Half Solids

U-106 Core 148 Segment \# 4 Riser 2 :

-Jar \# 10187 (250 mL jar size)

-227.1 gram net weight

* Subsampled Solids: Lower Half Solids

U-106 Core 148 Segment \# 4 Riser 2 ;

-Jar \# 10060 (125 mL jar size)

-194.3 gram net weight

Notes:

* A total of 421.4 grams of solids were collected. The dose rate through the drill string was $1.3 \mathrm{R} / \mathrm{hr}$. 


\section{WHC-SD-WM-DP-191, REV.I}

\section{U-106 Core 148 Segment \#5 Biser \#2}

Date: $\quad$ May 21, 1996

Sampler serial \#: $\quad 95-311 \mathrm{~S}$

Sample \#: $\quad 96-224$

Cask serial \#: C-1053

Logbook \#:

Labcore \#:

WHC-N-1342

S96T002820

Notes:
* $\quad$ Hot Cell Temp 79.5 degrees F. Humidity $28 \%$.
* Check weights: 20 gms $=20.00$ grams 500 gms $=500.01$ grams
* Dose rate through the drill string was $700 \mathrm{mR} / \mathrm{hr}$.
* Liner liquid: None observed.
* Sampler valve closed before and after cutting cables.
* Extruded sample and performed photography.

Sample information:

* No drainable liquid. Extruded approximately 11 inches of solids. The lower portion of solids were 5 inches in length, dark brown in color and the texture resembled a moist salt (M4). The upper portion was 6 inches in length, dark brown in color and the texture resembled a sludge slurry (D2). Subsampled solids in half segments.

Subsample information:

* Subsampled Solids: Upper Half Solids

U-106 Core 148 Segment \# 5 Riser 2:

$-J a r \# 10093$ (250 mL jar size)

-188.0 gram net weight

* Subsampled Solids: Lower Half Solids

U-106 Core 148 Segment \# 5 Riser 2:

-Jar \# 10066 (125 mL jar size)

-134.3 gram net weight

Notes:

* A total of 322.3 grams of solids were collected. The dose rate through the drill string was $700 \mathrm{mR} / \mathrm{hr}$. 
WHC-SD-WM-DP-191, REV. I

SAMPLE HANDLING 
WHC-SD-WM-DP-191, REV. 1

$\sigma$

THIS PAGE WAS INTENTIONALLY LEFT BLANK 
Analyst:

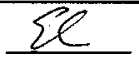

Instrument: BA000

Book \#

Method: LO-160-103 Rev/Mod $\mathrm{BO}$

Worklist Comment: U-106 C147 SEG \# 3 RISER 19 EXTRUSION

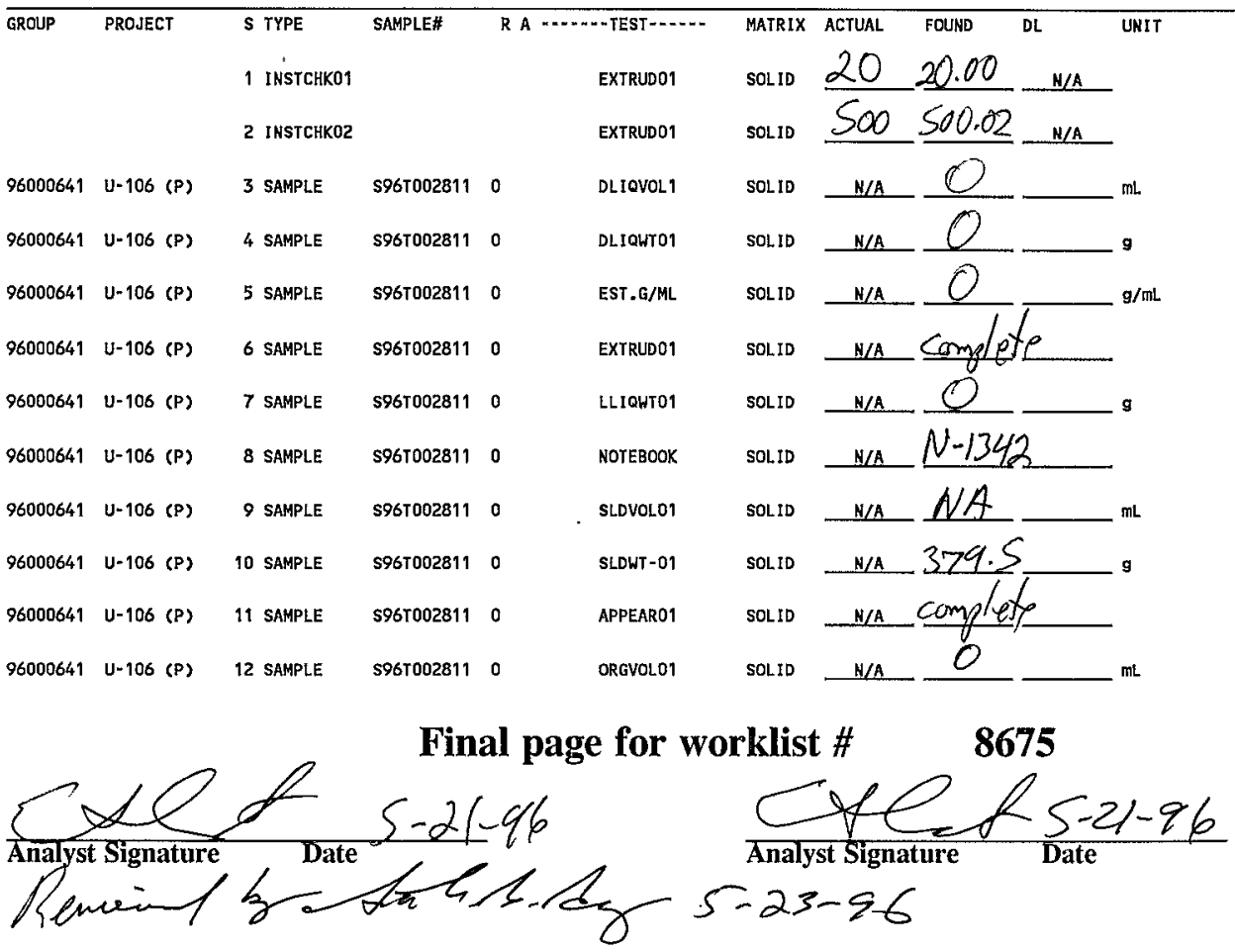

Data Entry Comments:

Units shown for $Q C$ (SPK \& STD) may not reflect the actual units. $D L=$ Detection Limit, $S=$ Worklist Slot Number, $R=$ Replicate Number, $A=$ Aliquot Code. 


\section{LABCORE Data Entry Template for Worklist\#}

\begin{tabular}{|c|c|c|c|}
\hline Analyst: & 4 & BA000 & Book \# NA \\
\hline
\end{tabular}

\section{Worklist Comment: U-106 C147 SEG \# 4 RISER 19 EXTRUSION}

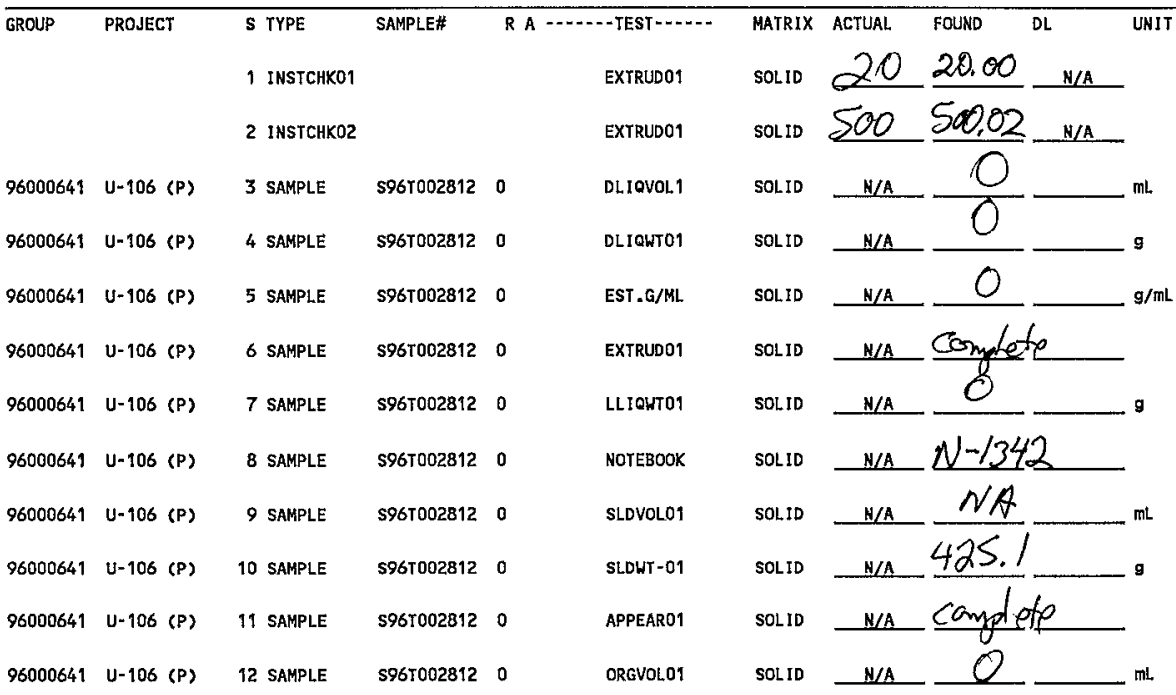

\section{Final page for worklist \# $\quad 8676$}

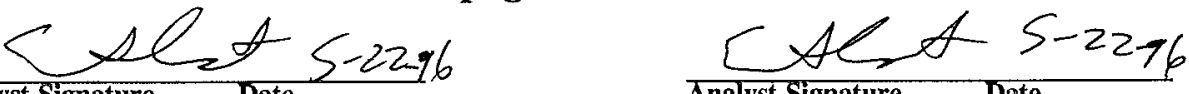
Analyst Signature Date

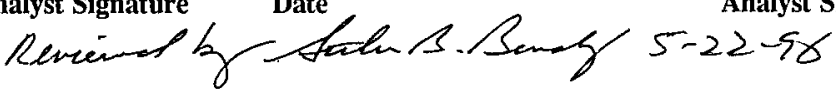

Data Entry Comments:

Units shown for $Q C$ (SPK \& STD) may not reflect the actual units. $D L=$ Detection Limit, $S=$ Worklist Slot Number,

$R=$ Replicate Number, $A=$ Aliquot Code. 


\section{LABCORE Data Entry Template for Worklist\#}

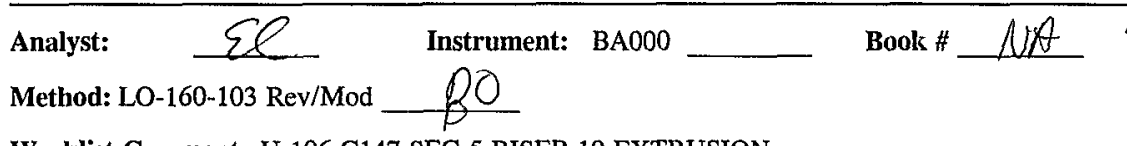

\section{Worklist Comment: U-106 C147 SEG 5 RISER 19 EXTRUSION}

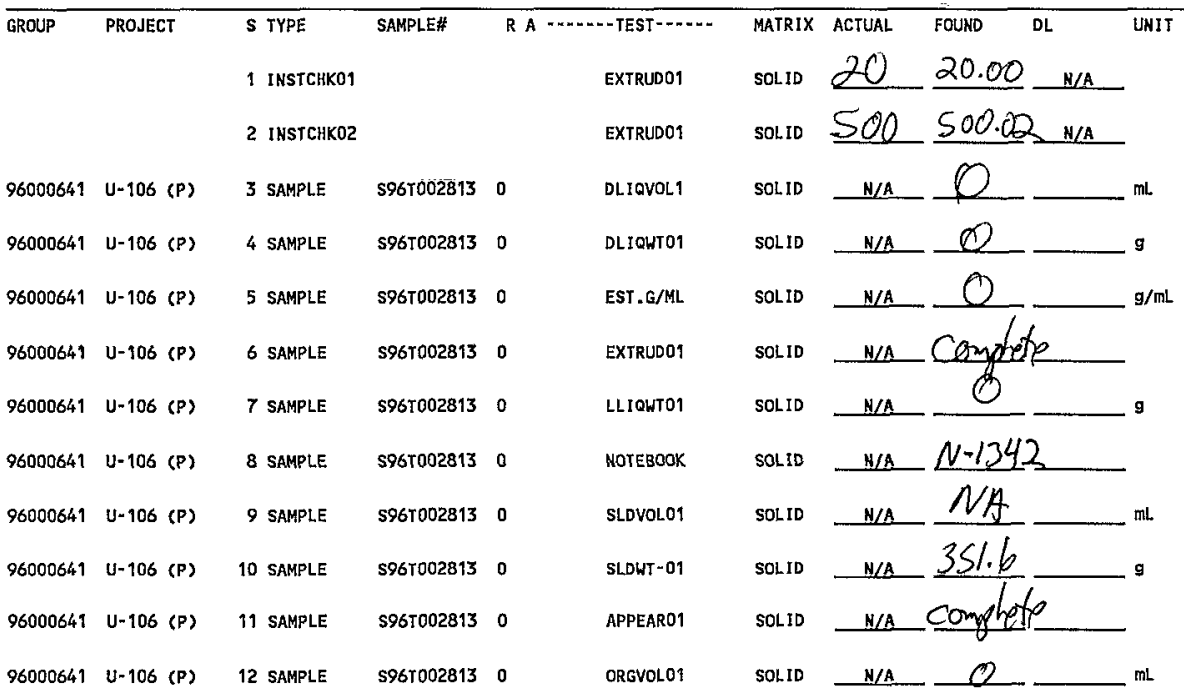

\section{Final page for worklist \# $\quad 8677$}

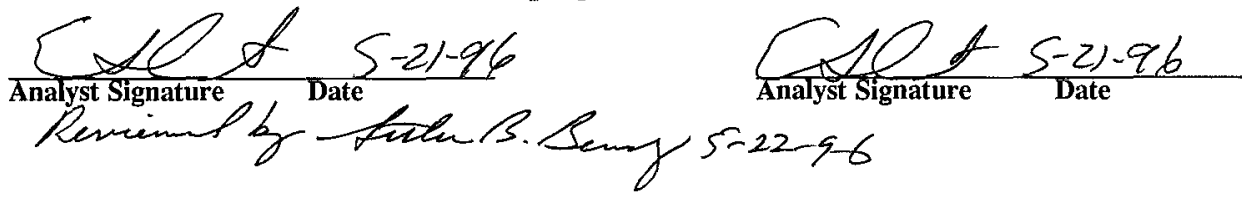

Data Entry Comments:

Units shown for $Q C$ (SPK \& STD) may not reflect the actual units. $D L=$ Detection Limit, $S=$ Worklist Slot Number, $R=$ Replicate Number, $A=$ Aliquot Code. 
WHG-SD-WM-DP-191, REV .।

worklistrpt Version $2.105 / 15 / 95$

Page: $\quad 1$

05/14/96 16:50

LABCORE Data Entry Template for Worklist\#

8678

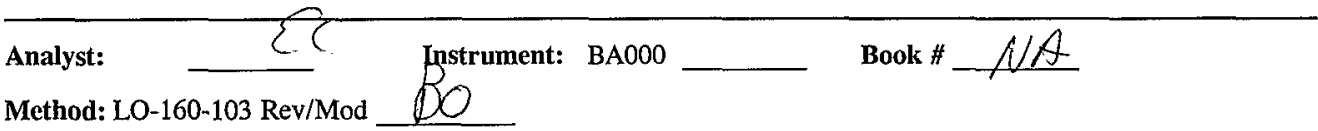

Worklist Comment: U-106 C148 SEG \# 3 RISER 2 EXTRUSION

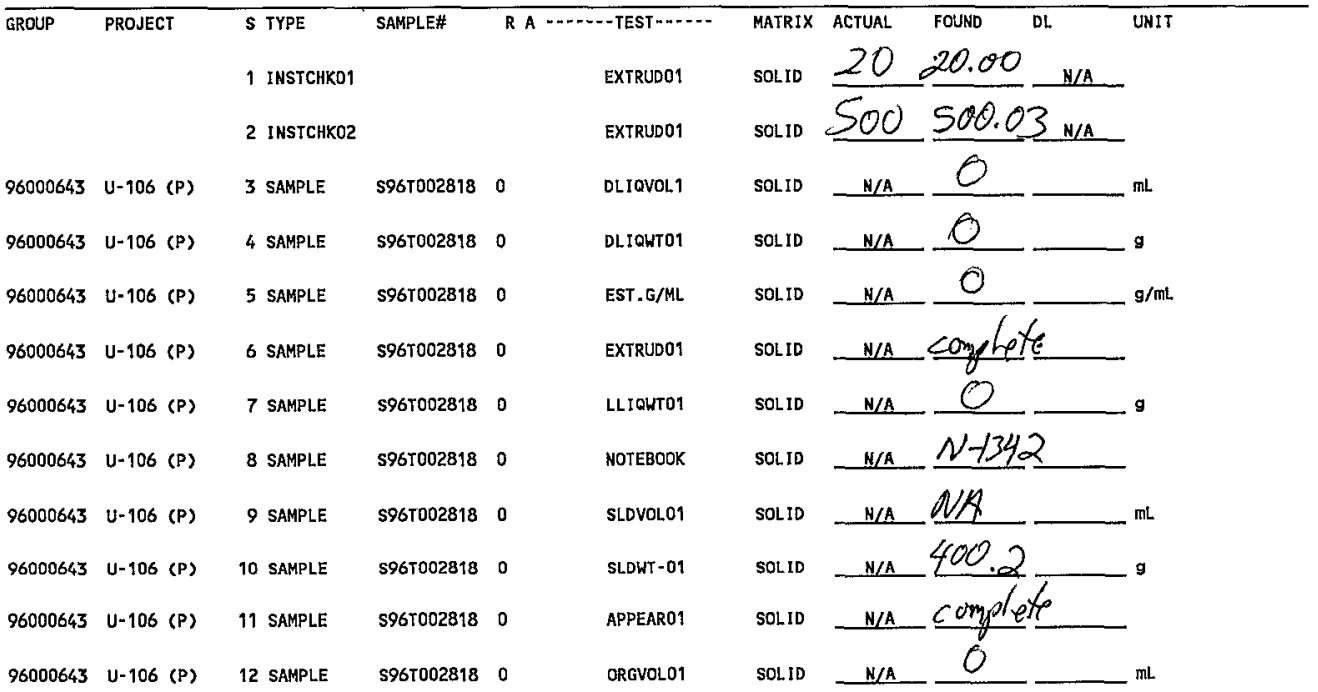

Final page for worklist \#

8678

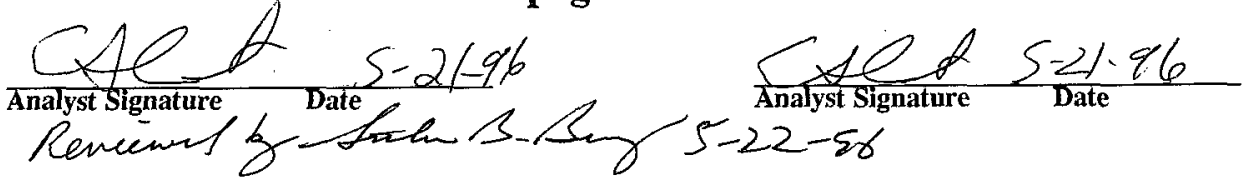

Data Entry Comments:

Units shown for QC (SPK \& STD) may not reflect the actual units. DL = Detection Limit, $S=$ Worklist Slot Number, $R=$ Replicate Number, $A=$ Aliquot Code.

141 


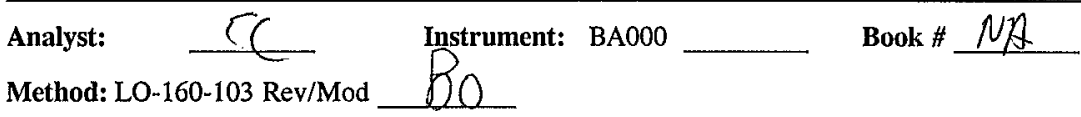

\section{Worklist Comment: U-106 C148 SEG \# 4 RISER 2 EXTRUSION}

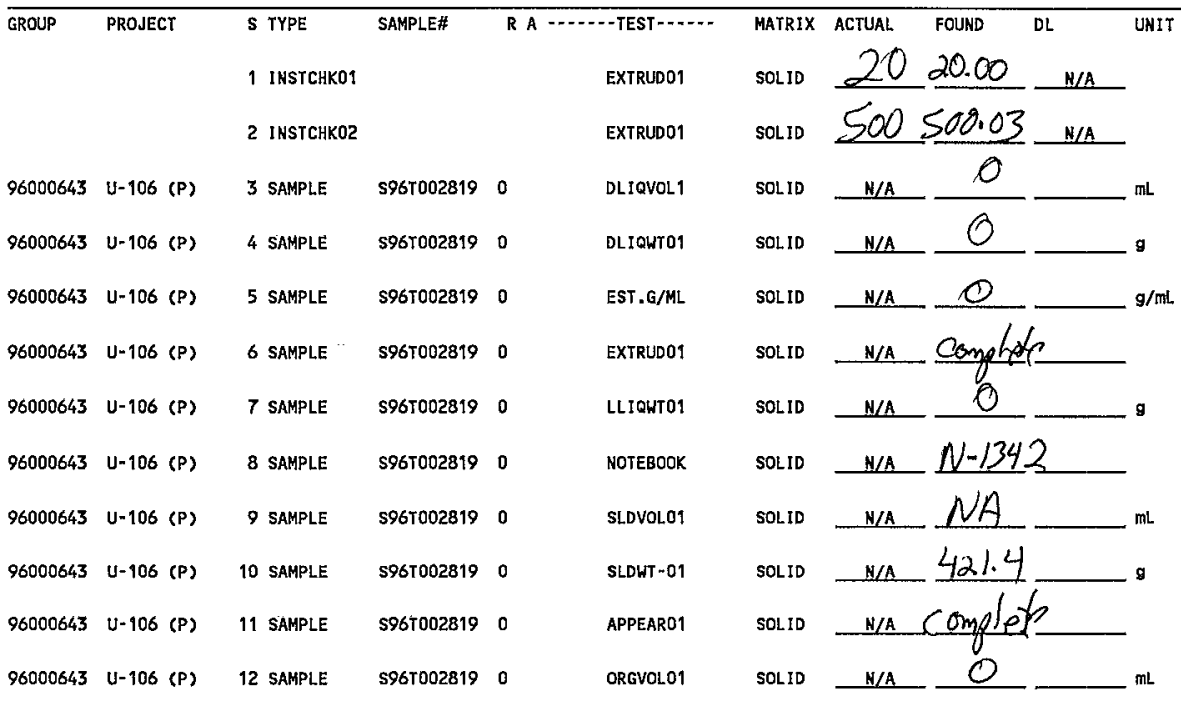

\section{Final page for worklist \# 8679}

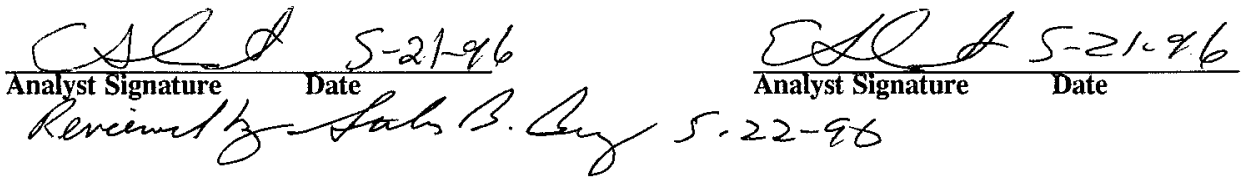

Data Entry Comments:

Units shown for $Q C$ (SPK \& STD) may not reflect the actual units. $D L=$ Detection Limit, $S=$ Worklist Slot Number, $R=$ Replicate Number, $A=$ Aliquot Code. 


\section{LABCŌRE Data Entry Template for Worklist\#}

Analyst: $\quad$ Instrument: BA000 Book \# NA

Method: LO-160-103 Rev/Mod

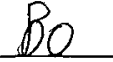

Worklist Comment: U-106 C148 SEG \# 5 RISER 2 EXTRUSION

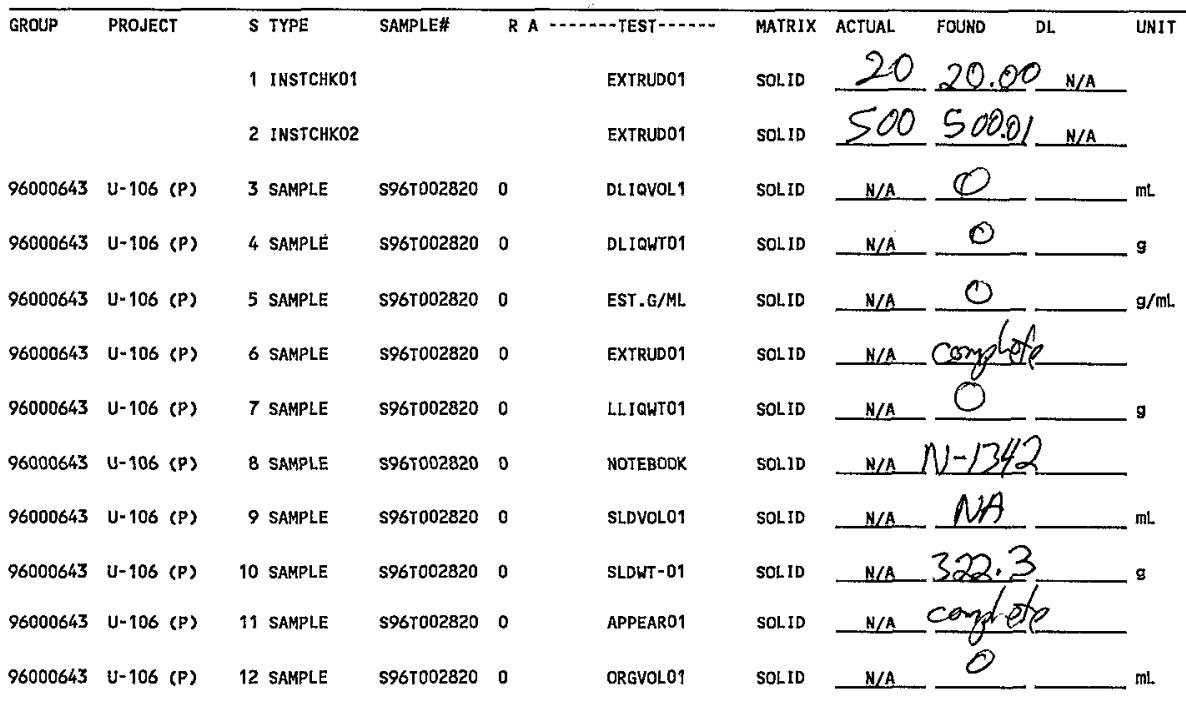

\section{Final page for worklist \# $\quad 8680$}

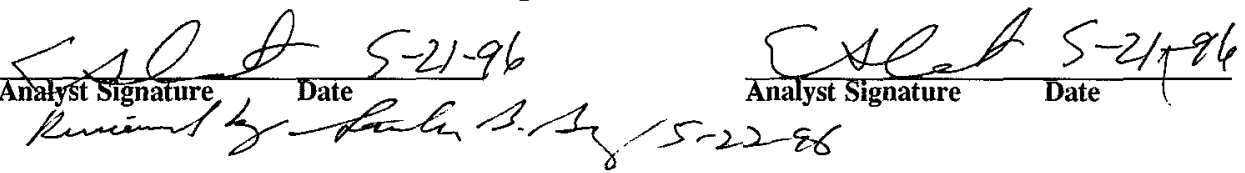

Data Entry Comments:

Units shown for $Q C$ (SPK \& STD) may not reflect the actual units. $D L=$ Detection Limit, $S=$ Worklist Slot Number,

$R=$ Replicate Number, $A=$ Aliquot Code. 


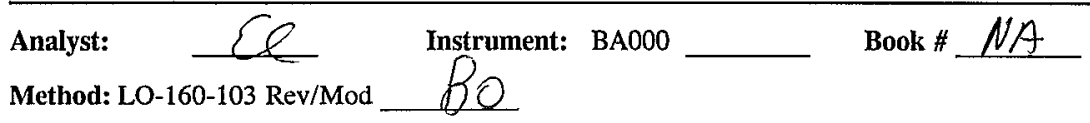

Worklist Comment: U-106 C148 SEG 2 RISER 2 EXTRUSION

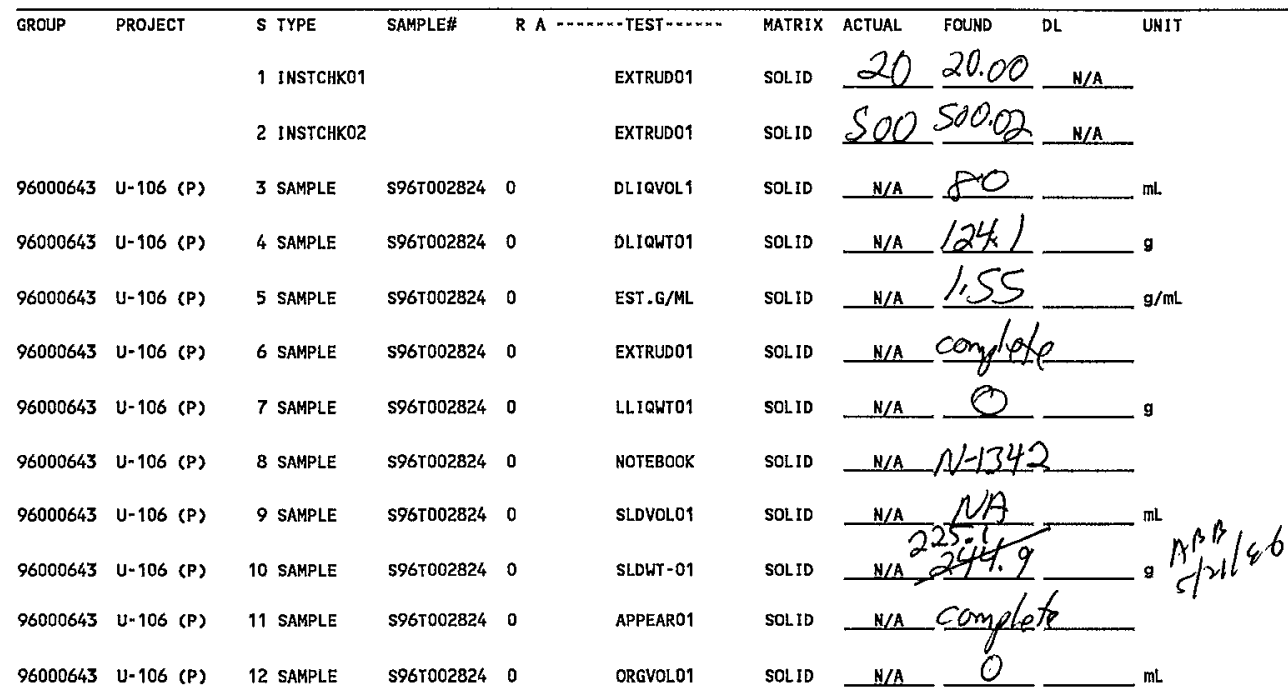

Final page for worklist \# $\mathbf{8 6 8 8}$

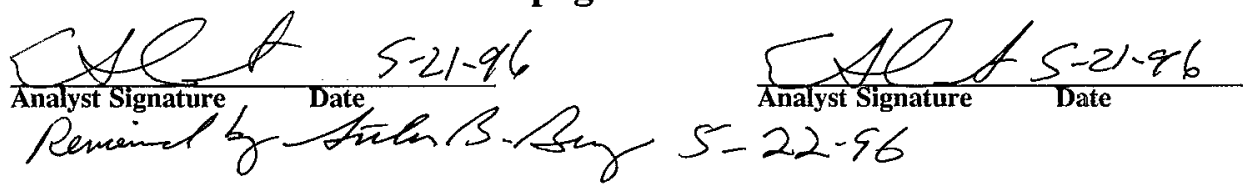

Data Entry Comments:

Units shown for $Q C$ (SPK \& STD) may not reflect the actual units. $D L=$ Detection Limit, $S=$ Worklist Slot Number,

$R=$ Replicate Number, $A=$ Aliquot Code. 


\section{LABCORE Data Entry Template for Worklist\#}

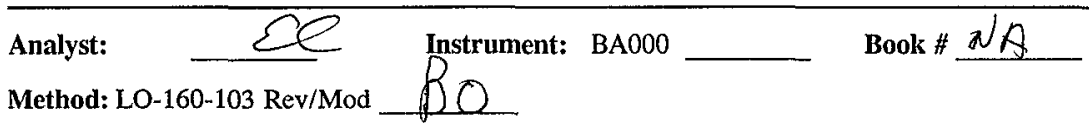

Worklist Comment: U-106 C147 SEG \# 1 RISER 19 EXTRUSION

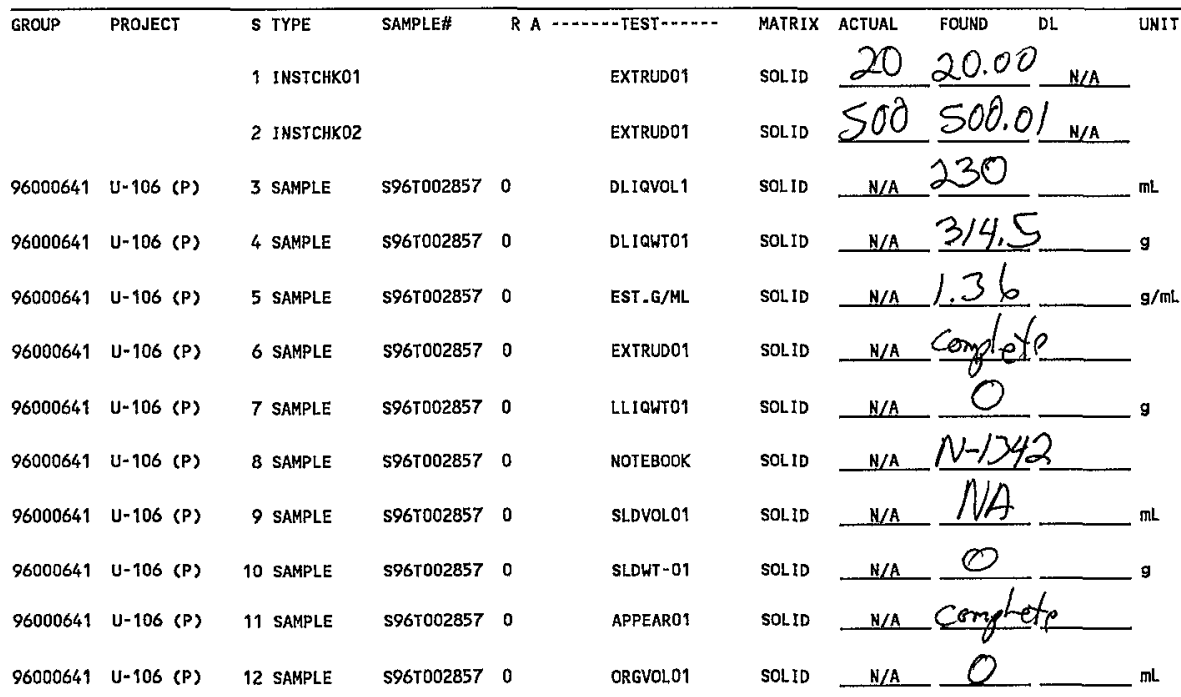

\section{Final page for worklist \# 8942}

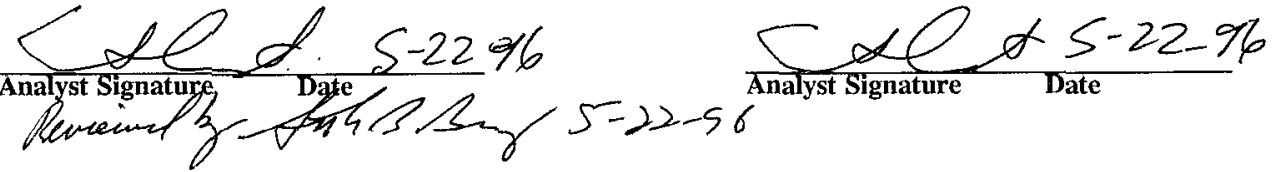

Data Entry Comments:

Units shown for QC (SPK \& STD) may not reflect the actual units. DL = Detection Limit, $S=$ Worklist Slot Number, Replicate Number, $A=$ Aliquot Code. 


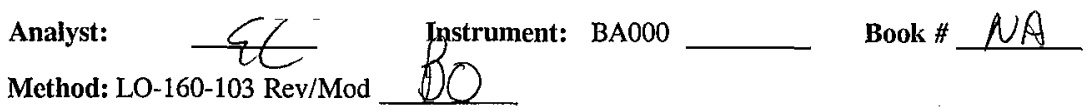

Worklist Comment: U-106 C147 SEG \# 2 RISER 19 EXTRUSION

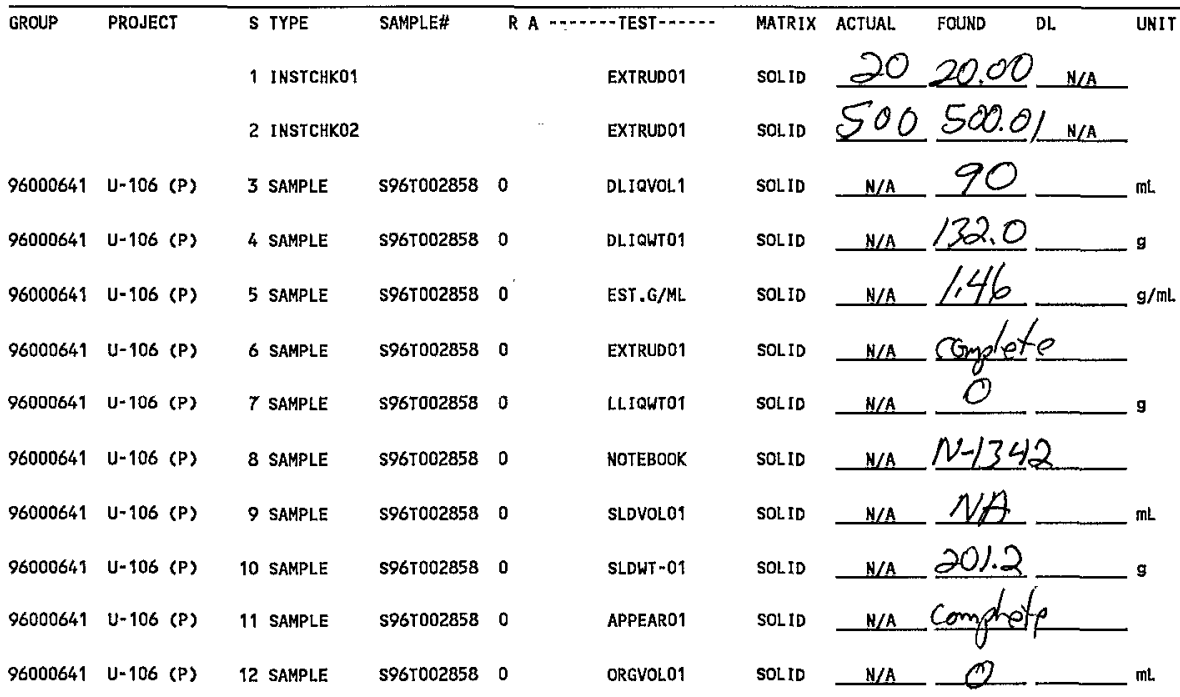

\section{Final page for worklist \# 8943}

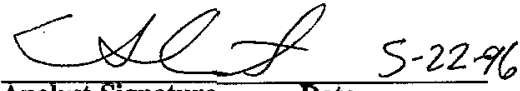

Analyst Signature Date

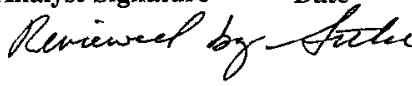

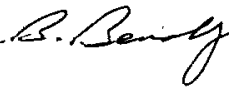

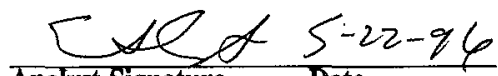

Analyst Signature

Data Entry Comments:

Units shown for $Q C$ (SPK \& STD) may not reflect the actual units. $D L=$ Detection Limit, $S=$ Worklist Slot Number, $R=$ Replicate Number, $A=$ Aliquot Code. 
WHC-SD-WY-DP-191, REV. 1

SAMPLE PREPARATIONS

147 
WHC-SD-WM-DP-191, REV. 1

THIS PAGE WAS INTENTIONALLY LEFT BLANK 


\section{WHG-SD-WM-DP-191, REV, I}

worklistrpt Version 2.1 05/15/95

06/03/96 10:03

\section{LABCORE Data Entry Template for Worklist\#}

Analyst: DCD / $/ \mathscr{C l C}$ Instrument: FUS01 Book \# N/A

Method: LA-549-141 Rev/Mod

Worklist Comment: TANK\#U-106,CORE 147,SEG 2UH,3UH,3LH,FOR FUSIONO1 RTS!

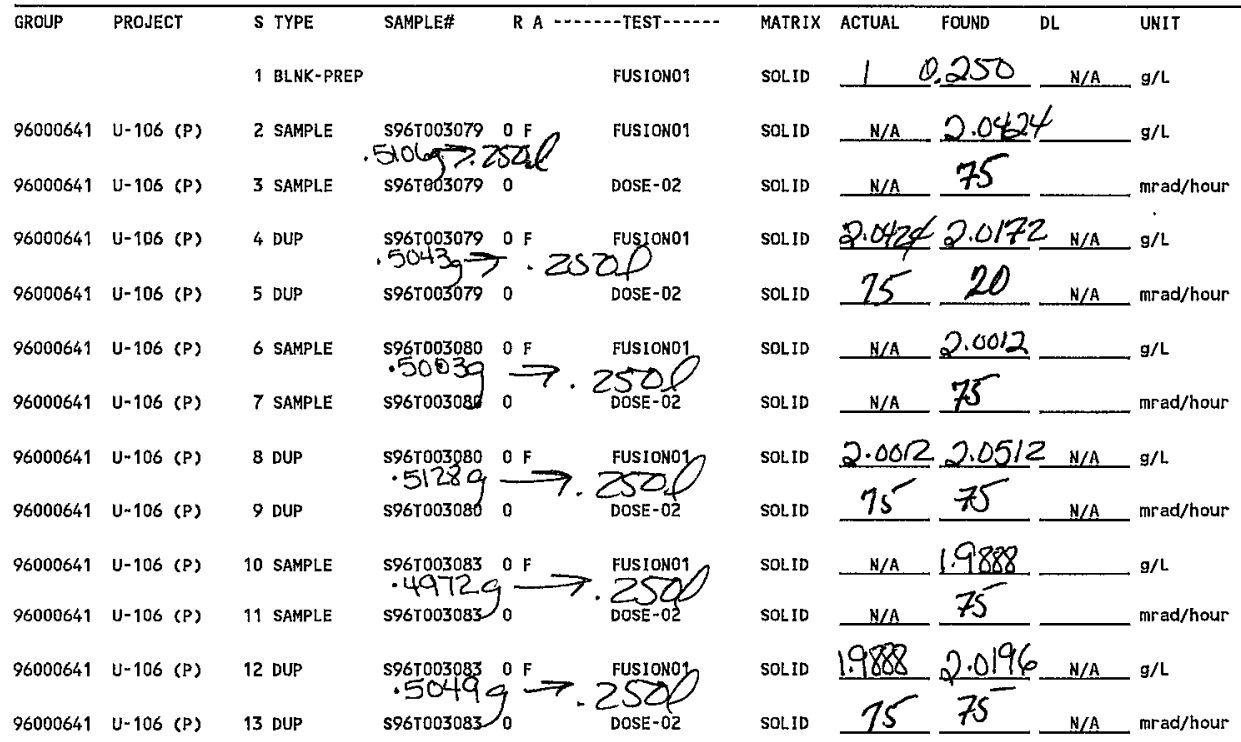

Final page for worklist \#

9468

Dawexdmantar $7-16-96$
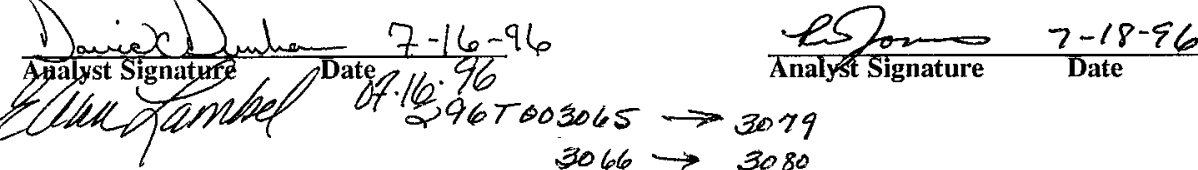

$0467003065>3079$

$3066 \rightarrow 3080$

$3070 \rightarrow 3083$

Data Entry Comments: Sm p44

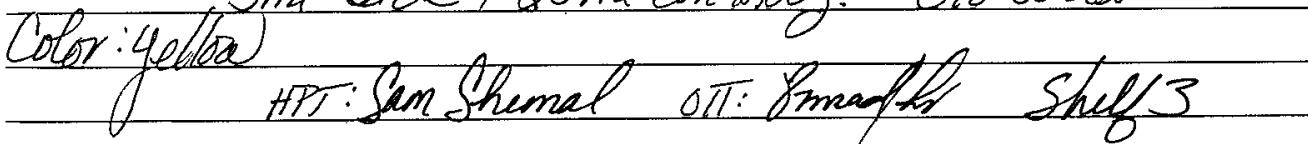

Units shown for $Q C$ (SPK \& STD) may not reflect the actual units. $D L=$ Detection Limit, $S=$ Worklist Slot Number, $R=$ Replicate Number, $A=$ Aliquot Code. 
Analyst: DCD/Hell Instrument: FUSO1 Book \# U/A

Method: LA-549-141 Rev/Mod

Worklist Comment: TANK\#U-106,CORE 147,SEG 4UH,4LH,5UH,5LH,FUSIONO1 RTS!

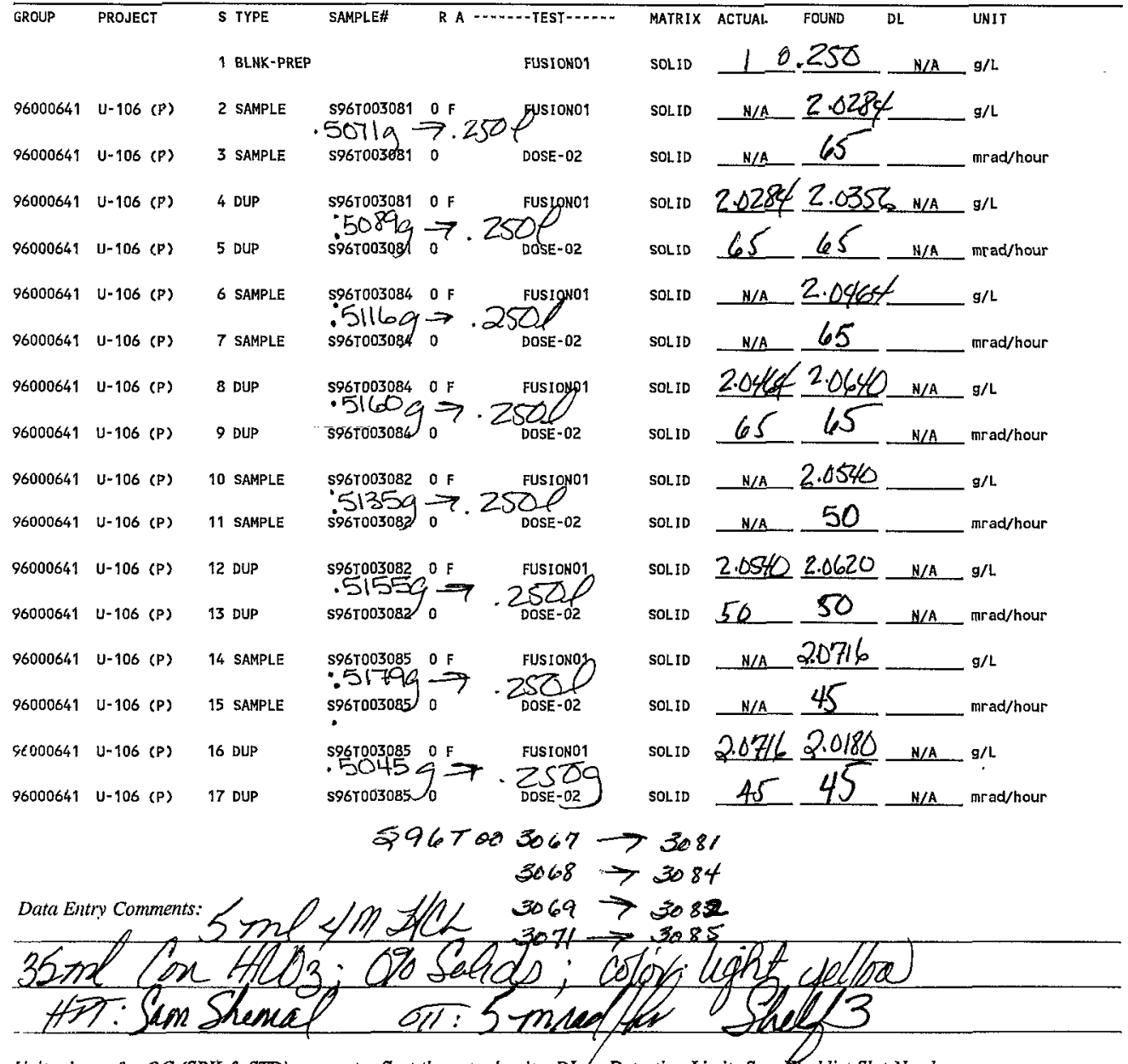

L'nits shown for $Q C$ (SPK \& STD) may not reflect the actual units. DL $=$ Detection Limit, $S=$ Worklist Slot Number, $R=$ Replicate Number, $A=$ Aliquot Code. 
worklistrpt Version 2.1 05/15/95

WHC-SD-WM-DP-191, REV, I

06/03/96 10:05

\section{LABCORE Data Entry Template for Worklist\#}

Page: 2

GROUP PROJECT

S IYPE

SAMPLE\#

R A - - - - - TEST -.....

MATRIX ACTUAL

FOUND

$\mathrm{DL}$

UNIT

9469

\section{Final page for worklist \# 9469}

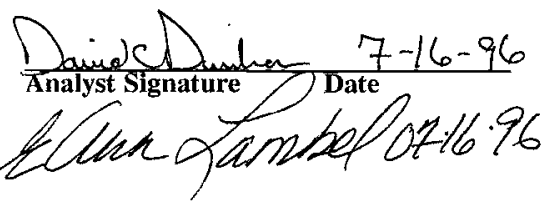

$\frac{x+20 z-7-18-96}{\text { Analyst Signature }}$

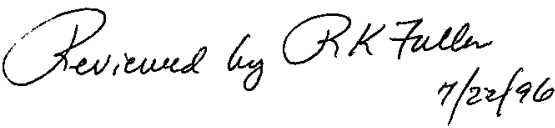

Data Entry Comments:

Units shown for $Q C$ (SPK \& STD) may not reflect the actual units. $D L=$ Detection Limit, $S=$ Worklist Slot Number,

$R=$ Replicate Number, $A=$ Aliquot Code. 
worklistrpt Version $2.105 / 15 / 95$

WHC-SD-WM-DP-191, REV. 1

06/03/96 10:11

LABCORE Data Entry Template for Worklist\#

Page: 1

9474

Analyst: Eff e Instrument: FUS01_ Book \#

Method: LA-549-141 Rev/Mod FO

Worklist Comment: TANK\#U-106,CORE 148,SEG 2UH,2LH,3UH, FUSION01 RTS!

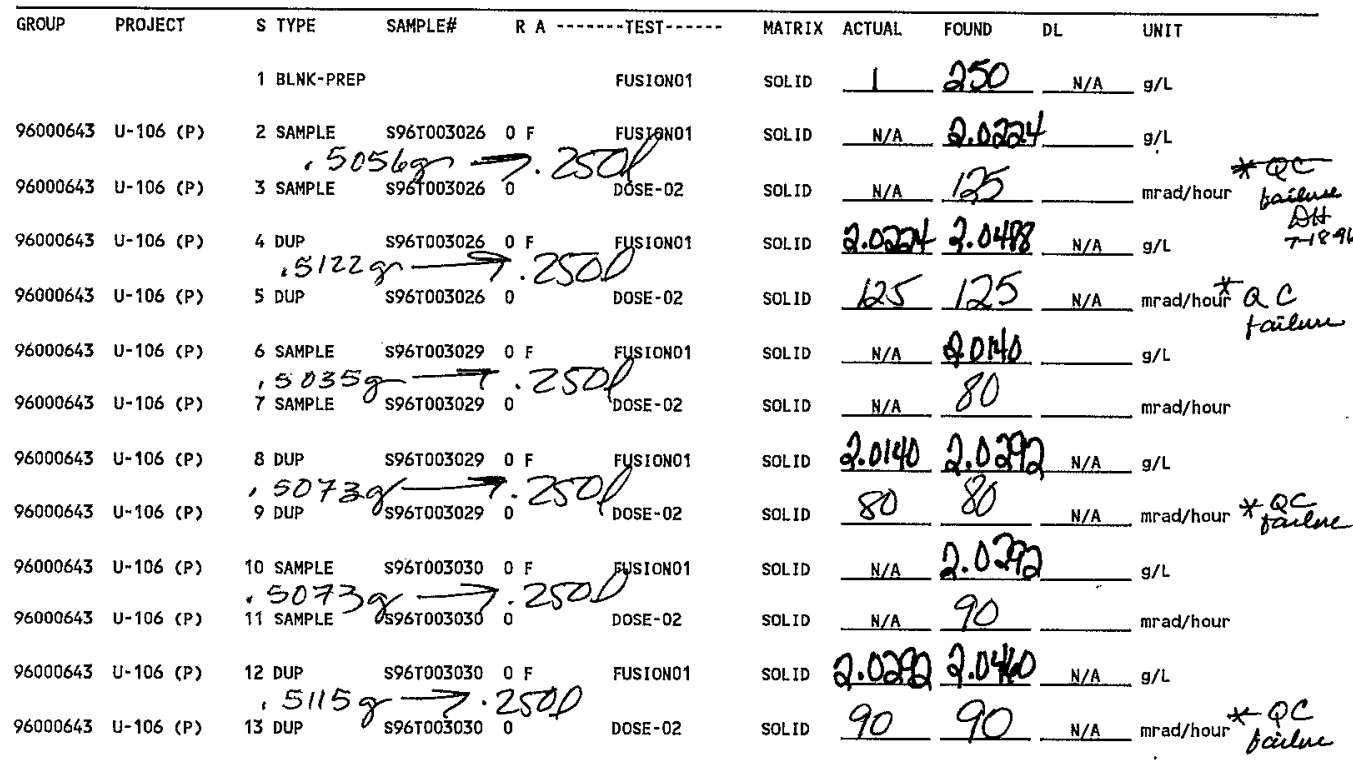

Final page for worklist \# 9474

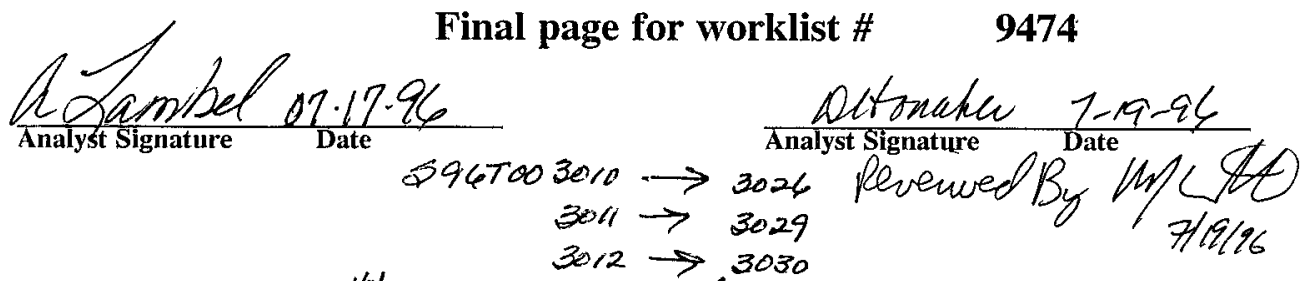

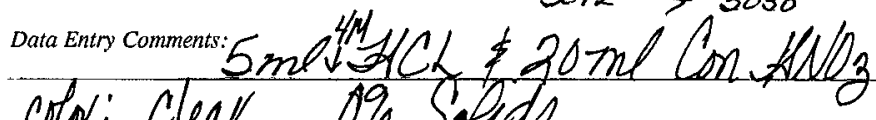

crow: clear 09 Solids

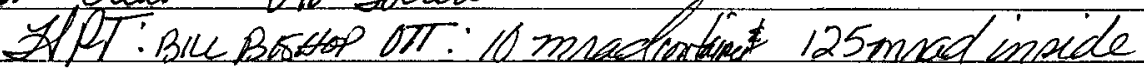

Units shown for QC (SPK \& STD) may not reflect the actual units, $D L=$ Detection Limit, $S=$ Worklist Slot Number, $R=$ Replicate Number, $A=$ Aliquot Code.

152 
Analyst: $\quad$ Instrument: FUS01

Method: LA-549-141 Rev/Mod $F-0$ Book $\# N A$

Worklist Comment: TANK\#U-106,CORE 148,SEG 3LH,4UH,4LH, FUSION01 RTS!

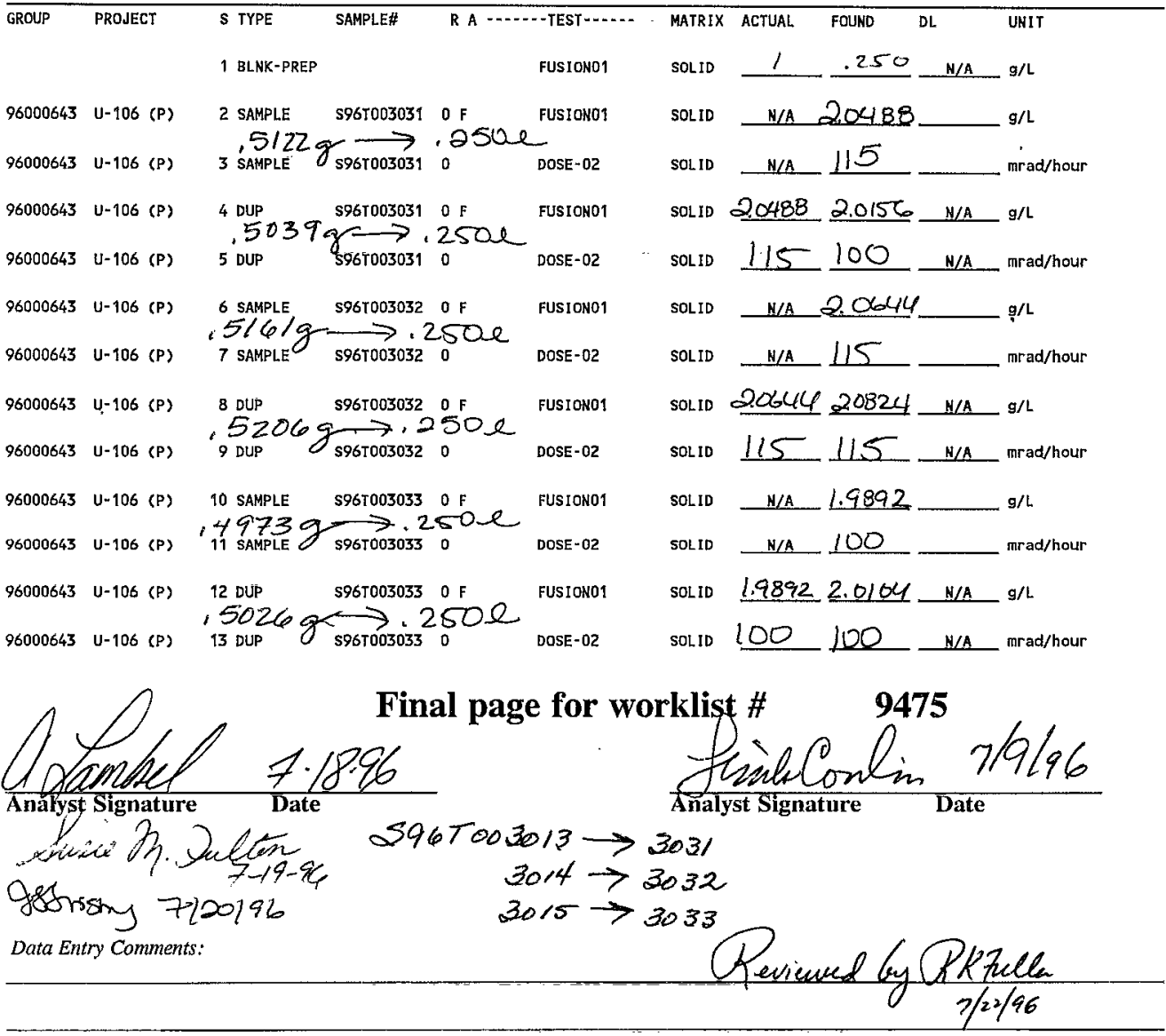

Units shown for $Q C$ (SPK \& STD) may not reflect the actual units. $D L=$ Detection Limit, $S=$ Worklist Slot Number, $R=$ Replicate Number, $A=$ Aliquot Code. 
Analyst: $S H$ Instrument: FUS01

Book \# 1 A

Method: LA-549-141 Rev/Mod FC

Worklist Comment: TANK\#U-106,CORE 148,SEG 5UH,5LH, FUSIONO1 RTS!

\begin{tabular}{|c|c|c|c|c|c|c|c|c|c|c|}
\hline GROUP & PROJECT & $\begin{array}{l}S \text { TYPE } \\
1 \text { BLNK-PREP }\end{array}$ & SAMPLE\# & R A $\cdots$ & - TEST $-\cdots$ & MATRIX & $\begin{array}{c}\text { ACTUAL } \\
1 \\
\end{array}$ & $\begin{array}{l}\text { FOUND } \\
250 \\
\text { + } 1\end{array}$ & $\begin{array}{l}D L \\
\text { DLA } \\
\text { N/A } \\
\end{array}$ & $\begin{array}{l}\text { UNIT } \\
\text { g/L }\end{array}$ \\
\hline 96000643 & $U-106(P)$ & $\begin{array}{l}2 \text { SAMPLE } \\
0.5 .043\end{array}$ & $\stackrel{\text { s96T003034 }}{\longrightarrow}, 2$ & OF & FUSIONO1 & SOLID & $\mathrm{N} / \mathrm{A}$ & & & $g / L$ \\
\hline 96000643 & $U-106$ (P) & 3 SAMPLE & S96T003034 & 0 & DOSE-02 & SOLID & N/A & 0 & & mrad/hour \\
\hline 96000643 & $U-106(P)$ & $\begin{array}{l}4 \text { DUP } \\
0.5 / 330\end{array}$ & s96T003034 & OF & FUSIONOI & SOLID & 20172 & 2.0532 & $N / A$ & $g / L$ \\
\hline 96000643 & $U-106(P)$ & 5 DUP & s96T003034 & 0 & DOSE-02 & SOLID & 50 & 5 & N/A & $\mathrm{mrad} / \mathrm{hour}$ \\
\hline 96000643 & $U-106(P)$ & $\begin{array}{l}6 \text { SAMPLE } \\
0.5360\end{array}$ & $\begin{array}{r}\text { s96T003035 } \\
\longrightarrow\end{array}$ & SOF & FUSIONO1 & SOLID & H/A & 2.144 & & $-g / L$ \\
\hline 96000643 & $U-106(P)$ & 7 SAMPLE & s96T003035 & 0 & DOSE -02 & SOL.tD & N/A & 30 & & mrad/hour \\
\hline 96000643 & $U=106(P)$ & $\begin{array}{l}8 \text { DUP } \\
0.523\end{array}$ & $\stackrel{596 T 003035}{7} 2$ & SOL & FUSIONOI & SOL ID & 2.144 & 20932 & N/A & $-g / L$ \\
\hline 96000643 & $U-106$ (P) & 9 DUP & S96T003035 & 0 & DOSE -02 & SOLID & 30 & & $N / A$ & mrad/hour \\
\hline
\end{tabular}

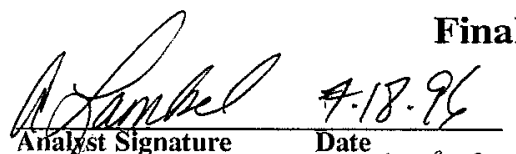

Analest Signature Date $7-19-4$

page for worklist \#

9476

Jussuisfishy $7 / 201915967003016 \rightarrow 3034$

$3017 \rightarrow 3035$

Data Entry Comments:

Pevicuedely QKzull

Units shown for QC (SPK \& STD) may not reflect the actual units, $D L=$ Detection Limit, $S=$ Worklist Slot Number,

$R=$ Replicate Number, $A=$ Aliquot Code. 
Analyst:

$2 \pi$

Instrument: FUS01

Book \#

Method: LA-549-141 Rev/Mod

Worklist Comment: U106 C147/148 COMP FUSION01 SKB

\begin{tabular}{|c|c|c|c|c|c|c|c|c|c|c|}
\hline GROUP & PROJECT & $\begin{array}{l}S \text { TYPE } \\
1 \text { BLNK-PREP }\end{array}$ & SAMPLE\# & RA & $\begin{array}{l}\text { FUSION01 } \\
\text { FEST }\end{array}$ & $\begin{array}{l}\text { MATRIX } \\
\text { SOLID }\end{array}$ & $\begin{array}{r}\text { ACTUAL } \\
1 \\
\end{array}$ & $\begin{array}{l}\text { FOUND } \\
0.250 \\
\end{array}$ & $\begin{array}{l}\mathrm{DL} \\
\mathrm{N} / \mathrm{A} \\
\end{array}$ & $\begin{array}{l}\text { UHIT } \\
-9 / L\end{array}$ \\
\hline 96000641 & $U-906(P)$ & $\sqrt{2}$ SAMPLE & $\begin{array}{l}\text { \$96T003878 } \\
, 5170\end{array}$ & $\stackrel{0 F}{\rightarrow}$ & $.250 l^{\text {Fusfonor }}$ & SOLID & N/A & 2.068 & & $g / L$ \\
\hline 96000641 & $U-106(P)$ & 3 SAMPLE & S96T003878 & $O F$ & DOSE-02 & SOLID & N/A & 80 & 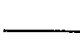 & mrad/hour \\
\hline 96000641 & $U-106(P)$ & $\checkmark 4$ DUP & $\begin{array}{r}5967003878 \\
.5230\end{array}$ & $\stackrel{0 F}{\rightarrow}$ & $.250 e^{\text {Fusson01 }}$ & SOLID & 2.068 & 2,092 & $\mathrm{~N} / \mathrm{A}$ & $g / L$ \\
\hline 96000641 & $U-106(P)$ & 5 DUP & S96T003878 & $O F$ & DOSE-02 & SOLID & 80 & 80 & N/A & mrad/hour \\
\hline 96000643 & $U-106(P)$ & 6 SAMPLE & $\begin{array}{c}5961003884 \\
, 5127\end{array}$ & $\stackrel{0 F}{\rightarrow}$ & $.250 l^{\text {Fusigno1 }}$ & SOLID & N/A & 2,051 & & $-9 / 6$ \\
\hline 96000643 & $U-106(P)$ & 7 SAMPLE & s96T003884 & $O F$ & DOSE $=02$ & SOLID & N/A & 80 & 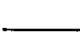 & mrad/hour \\
\hline 96000643 & $U-106(P)$ & 8 DUP & $\begin{array}{r}\text { s96t003884 } \\
.57 / 4\end{array}$ & $\rightarrow$ & $.250 P^{\text {Fusiono1 }}$ & SOLID & 2,051 & 2,285 & N/A & $\mathrm{g} / \mathrm{h}$ \\
\hline 96000643 & $U-106(P)$ & 9 DUP & S96T003884 & $6 \mathrm{~F}$ & DOSE-02 & SOLID & 80 & 75 & N/A & mrad/hour \\
\hline
\end{tabular}

\section{Final page for worklist \# 10956}

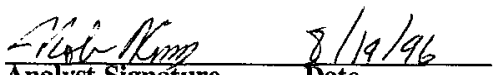

Analyst Signáture
Date

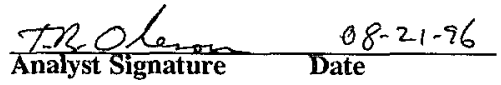

$$
\begin{array}{r}
0967003876 \rightarrow 3878 \\
3882 \rightarrow 3884
\end{array}
$$

Data Entry Comments:

submittef by RsuleCow s/20/96

Units shown for $Q C$ (SPK \& STD) may not reflect the actual units. $D L=$ Detection Limit, $S=$ Worklist Slot Number, $R=$ Replicate Number, $A=$ Aliquot Code. 


\section{LABCORE Data Entry Template for Worklist\#}

Page: $\quad I$

Analyst: $\quad$ DCD $\quad$ Instrument: $\mathrm{H} 2 \mathrm{O01} \quad$ Book \# $\mu / \AA$

Method: LA-504-101 Rev/Mod

$6 \cdot 0$

Worklist Conment: TANK\#U-106,CORE 147,SEG 2UH,3UH, H2ODIG01 RTS!

\begin{tabular}{|c|c|c|c|c|c|c|c|c|c|c|}
\hline \multirow[t]{2}{*}{ GROUP } & PROJECT & S TYPE & SAMPLE\# & \multicolumn{2}{|c|}{ R A $\cdots$} & \multirow{2}{*}{$\begin{array}{l}\text { MATRIX } \\
\text { SOLID }\end{array}$} & \multirow{2}{*}{$\begin{array}{c}\text { ACTUAL } \\
1 \\
\end{array}$} & \multirow{2}{*}{$\begin{array}{l}\text { FOLND } \\
.100 \\
\end{array}$} & \multirow{2}{*}{$\begin{array}{l}\mathrm{DL} \\
\mathrm{N} / \mathrm{A} \\
\end{array}$} & \multirow{2}{*}{$\begin{array}{l}\text { UNIT } \\
-\mathrm{g} / \mathrm{L}\end{array}$} \\
\hline & & 1 BLNK-PREP & & & H2ODIG01 & & & & & \\
\hline 96000641 & $U-106(P)$ & $\begin{array}{l}\text { 2 SAMPLE } \\
0.509 / \mathrm{g}\end{array}$ & $\stackrel{596 \mathrm{~T} 003093}{\longrightarrow} .100$. & $\stackrel{0}{\rho} \mathrm{W}^{-1}$ & H2001G01 & SOLID & N/A & 5.041 & & $g / L$ \\
\hline 96000641 & $U-106(P)$ & 3 SAMPLE & S96T003093 & 0 & DOSE-02 & SOLID & N/A & & & mrad/hour \\
\hline 96000641 & $U-106(P)$ & $\begin{array}{l}4 \text { DUP } \\
0.5 / 130\end{array}$ & $\stackrel{\text { s96T003093 }}{\longrightarrow}, 100$ & $\stackrel{0}{\rho}^{\mathrm{W}}$ & H20D1G01 & SOLID & 5.091 & 5. & N/A & $g / L$ \\
\hline 96000641 & $U=106(P)$ & 5 DUP & s96T003093 & 0 & DOSE-02 & SOLID & 75 & & $\mathrm{H} / \mathrm{A}$ & mrad/hour \\
\hline 96000641 & $U-106(P)$ & $\begin{array}{l}6 \text { SAMPLE } \\
0.5149 \mathrm{~g}\end{array}$ & $\begin{array}{r}5961003094 \\
-5.10\end{array}$ & OH & H2ODIGO1 & SOLID & N/A & 5.149 & & $g / L$ \\
\hline 96000641 & $U-106(P)$ & 7 SAMPLE & S96T003094 & 0 & DOSE- 02 & SOLID & N/A & & & $\mathrm{mrad} /$ hour \\
\hline 96000641 & $U=106(P)$ & $\begin{array}{l}8 \text { DUP } \\
0.5022\end{array}$ & $\begin{array}{r}5961003094 \\
-100\end{array}$ & $\stackrel{0}{O}^{\mathrm{W}}$ & $H 2001601$ & SOLID & 5.149 & $\frac{5.022}{75}$ & N/A & $g / L$ \\
\hline 96000641 & $U-106(P)$ & 9 DUP & $\$ 987003094$ & 0 & DOSE-02 & SOLID & & & $N / A$ & $\mathrm{mrad} / \mathrm{hour}$ \\
\hline
\end{tabular}

\section{Final page for worklist \# 9471}

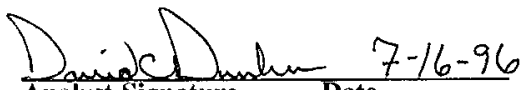

Analyst Signature Date

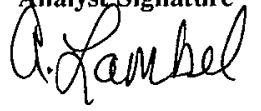

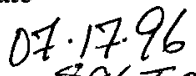

$\$ 967003065 \rightarrow 3093$ $3066 \rightarrow 3094$

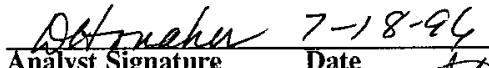
Analyst Signaturg Revenved By $m<\notin 0$ $7 / 18 / 96$

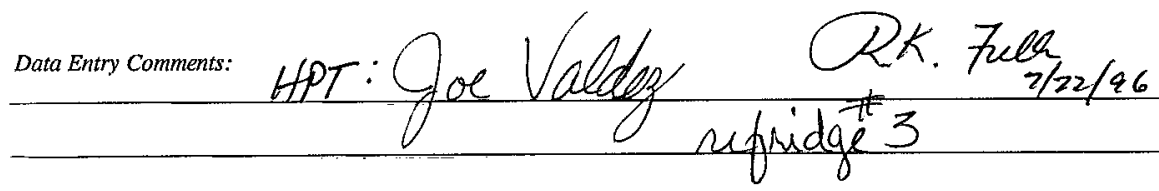

Units shown for QC (SPK \& STD) may not reflect the actual units. $D L=$ Detection Limit, $S=$ Worklist Slot Number,

$R=$ Replicate Number, $A=$ Aliquot Code. 
worklistrpt Version $2.105 / 15 / 95$

WHC-SD-WM-DP-191, REV. 1

06/03/96 10:08

LABCORE Data Entry Template for Worklist\#

Page: $\quad I$

9472

Analyst: $\quad \mathrm{DCD} \quad$ Instrument: $\mathrm{H} 2001+$ Book \# $\mathrm{N} / \mathrm{A}$

Method: LA-504-101 Rev/Mod E.O

Worklist Comment: TANK\#U-106,CORE 147,SEG 3LH,4UH, H2ODIG01 RTS!

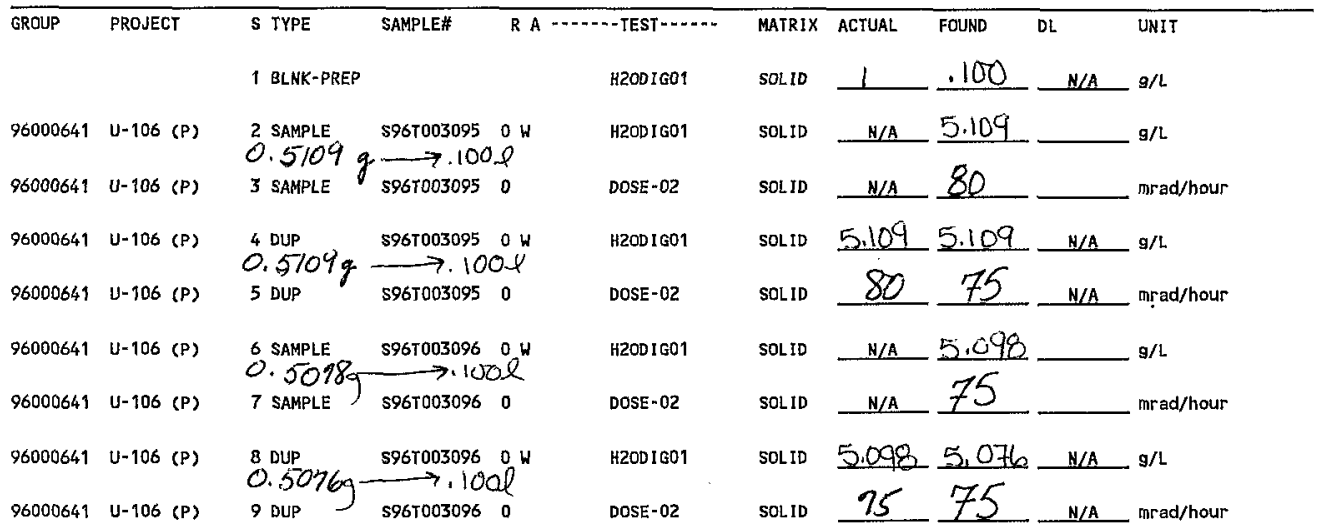

Final page for worklist \# 9472

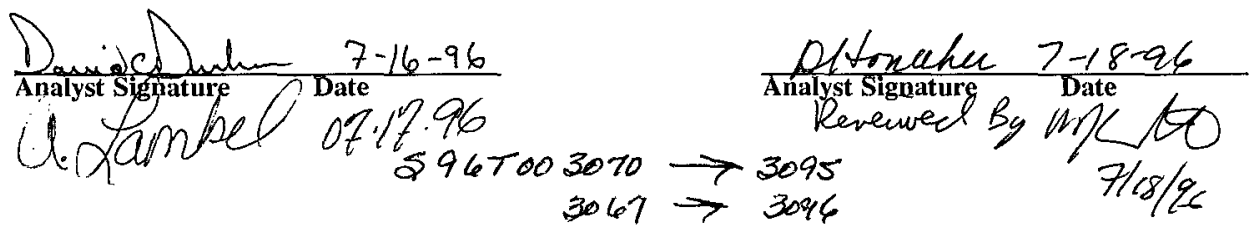

Data Entry Comments:

APT:

RKzule

wefridget $37 / 22 / 96$

Units shown for $Q C$ (SPK \& STD) may not reflect the actual units. $D L=$ Detection Limit, $S=$ Workist Slot Number, $R=$ Replicate Number, $A=$ Aliquot Code.

157 
Analyst: $\quad D C D, K N T$ Instrument: $\mathrm{H} 2001 \ldots$ Book $\# / A$

Method: LA-504-101 Rev/Mod E.O

Worklist Comment: TANK\#U-106,CORE 147,SEG 4LH,5UH,5LH, H2ODIG01 RTS!

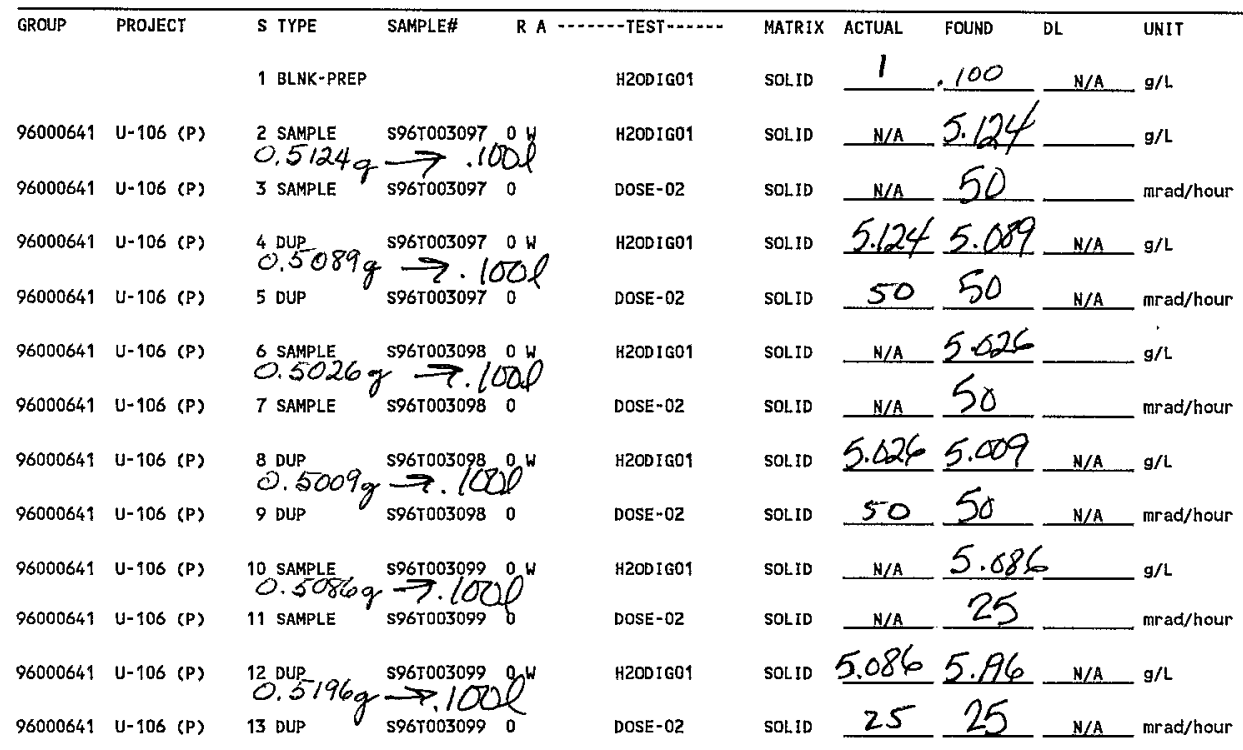

\section{Final page for worklist \# 9473}

$1 D_{\text {asuiged }}$ 7-16-96

Analyst Signature Date

Lim Dhonas

?

$7-18-96 \leqslant 967003068$

$3069 \rightarrow 3098$.

$3011 \div 3099$
25

Analfst Signature

$7-18-86$

Date Revewived By in/ $7 / 19 / 96$

Data Entry Comments:
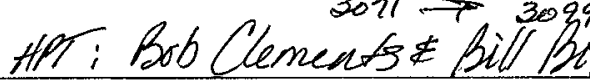

hop

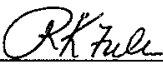

Pkzuel 2/22/96

Units shown for QC (SPK \& STD) moy not reflect the actual units. $D L=$ Detection Limit, $S=$ Worklist Slot Number, $R=$ Replicate Number, $A=$ Aliquot Code. 


\section{LABCORE Data Entry Template for Worklist\#}

Analyst: Instrument: H2O01 Book \#

Method: LA-504-101 Rev/Mod

$E \cdot O$

Worklist Comment: TANK\#U-106,CORE 148,SEG 2UH,2LH, H2ODIG01 RTS!

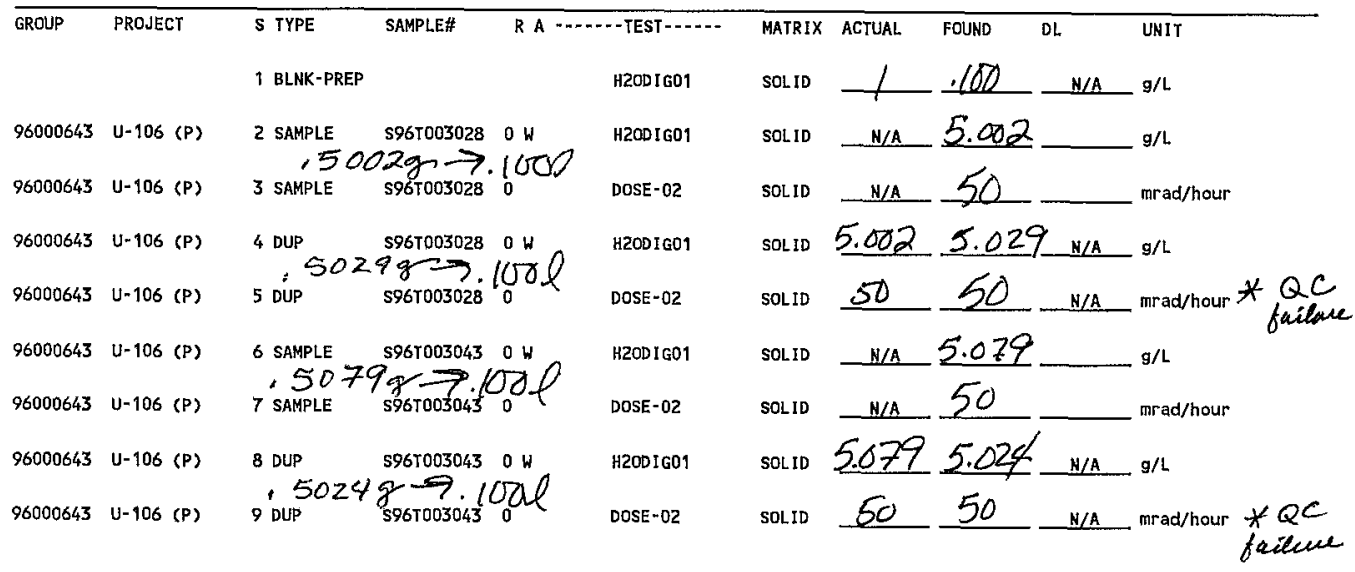

Final page for worklist \#

9479

4 Analyst Signature

Rem Lhomas

$$
\begin{array}{r}
396 T 003010 \rightarrow 3058 \\
3011 \rightarrow 3043
\end{array}
$$

Analyst Signature Date Reveuved $B$ by (n) 7196

Data Entry Comments:

HP. BILC BISETP

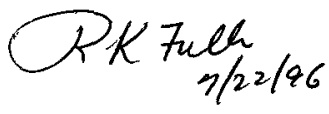

Units shown for QC (SPK \& STD) moy not reflect the actual units. $D L=$ Detection Limit, $S=$ Worklist Slot Number, $R=$ Replicate Number, $A=$ Aliquot Code. 


\section{LABCORE Data Entry Template for Worklist\#}

Analyst:

Instrument: $\mathrm{H} 2 \mathrm{OO}$

Book \#

Method: LA-504-101 Rev/Mod E-O

Worklist Comment: TANK\#U-106,CORE 148,SEG 3UH,3LH, H2ODIG01 RTS!

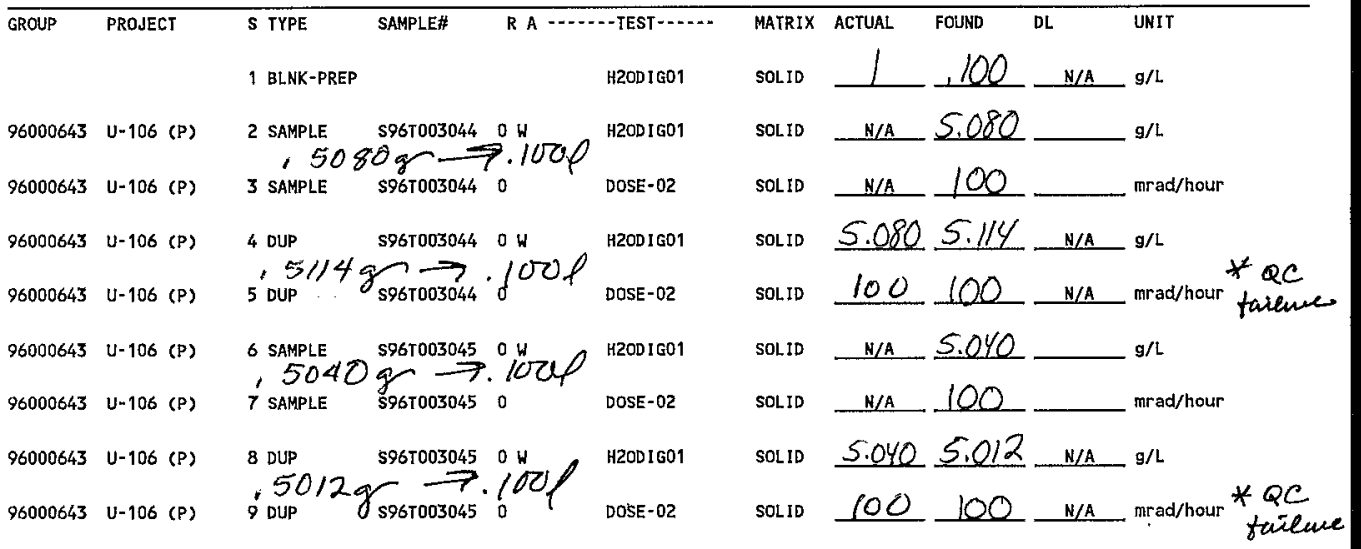

\section{Final page for worklist \# 9480}

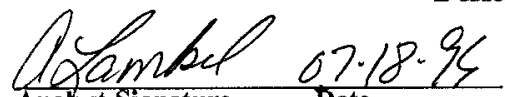

Anakyst Signature Date

Him 2 7homos $78-96$ $\$ 96 T 003012 \rightarrow 3044$

Dotoraken 7-18-96 Analyst Signature Date Bevevurey By $3013 \rightarrow 3045$ 
WHC-SD-WM-DP-191, REV. I

worklistrpt Version $2.105 / 15 / 95$

Page: $\quad 1$

06/03/96 10:19

LABCORE Data Entry Template for Worklist\#

9481

Analyst: Le Instrument: H2O01_ Book\#

Method: LA-504-101 Rev/Mod E-O

Worklist Comment: TANK\#U-106,CORE 148,SEG 4UH,4LH, H2ODIG01 RTS!

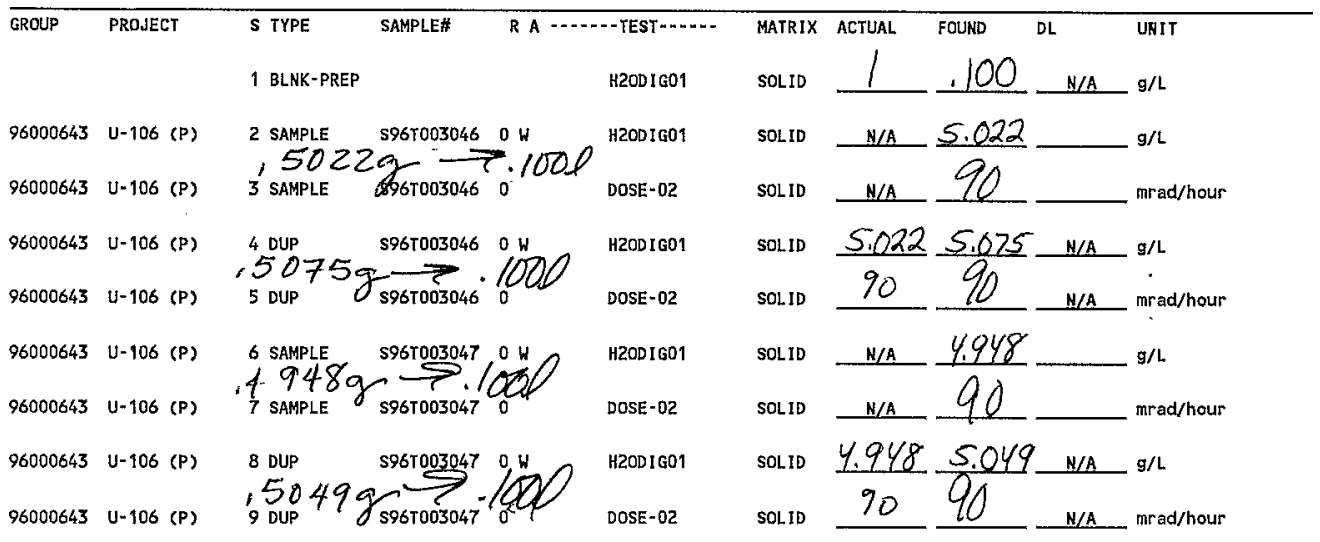

Final page for worklist \# 9481

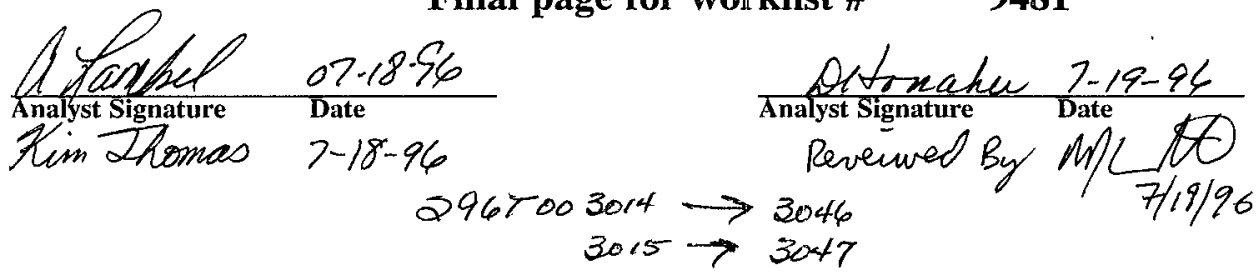

PKFull n/20/96

Data Entry Comments:

Clements

$0 \pi{ }^{\prime} 10$

Units shown for $Q C$ (SPK \& STD) may not reflect the actual units. $D L=$ Detection Limit, $S=$ Worklist Slot Number, $R=$ Replicate Number, $A=$ Aliquot Code.

161 
Analyst: $\quad E A L_{-}$Instrument: H2O01
Method: LA-504-101 Rev/Mod
Worklist Comment: TANK\#U-106,CORE 148,SEG 5UH,5LH, H2ODIG01 RTS!

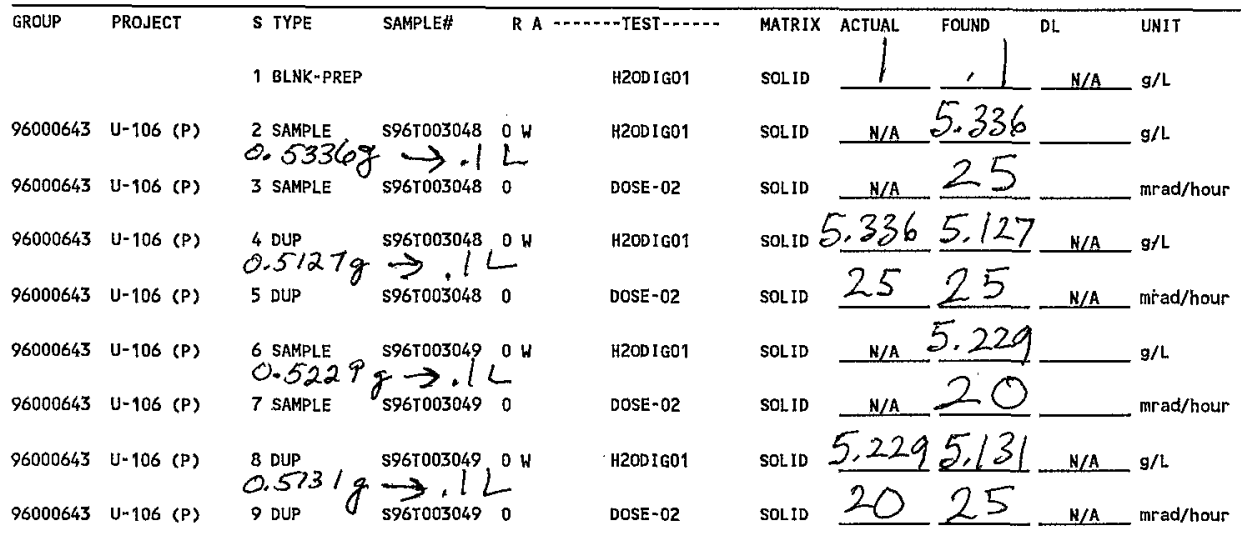

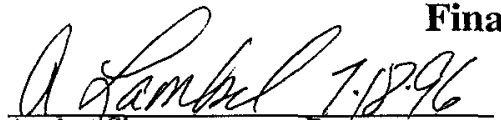

Analyst Signature

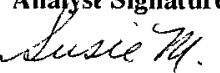

zetion

Date

\section{for worklist \#}

9482

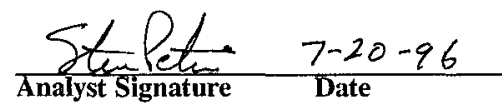

\section{$7-17-96$}

$\$ 96700$

\section{$3016 \rightarrow 3048$ \\ $3017 \rightarrow 30+9$}

Data Entry Comments:

$\frac{\text { Revieneed by RKFulle }}{1 / 22 / 46}$

Units shown for QC (SPK \& STD) may not reflect the actual units. $D L=$ Detection Limit, $S=$ Worklist Slot Number,

$R=$ Replicate Number, $A=$ Aliquot Code. 


\section{LABCORE Data Entry Template for Worklist\#}

Analyst:

Instrument: $\mathrm{H} 2 \mathrm{OO}$

Book \#

Method: LA-504-101 Rev/Mod

Worklist Comment: U106 C147/148 COMP H2ODIG01/H2OICP01 SKB

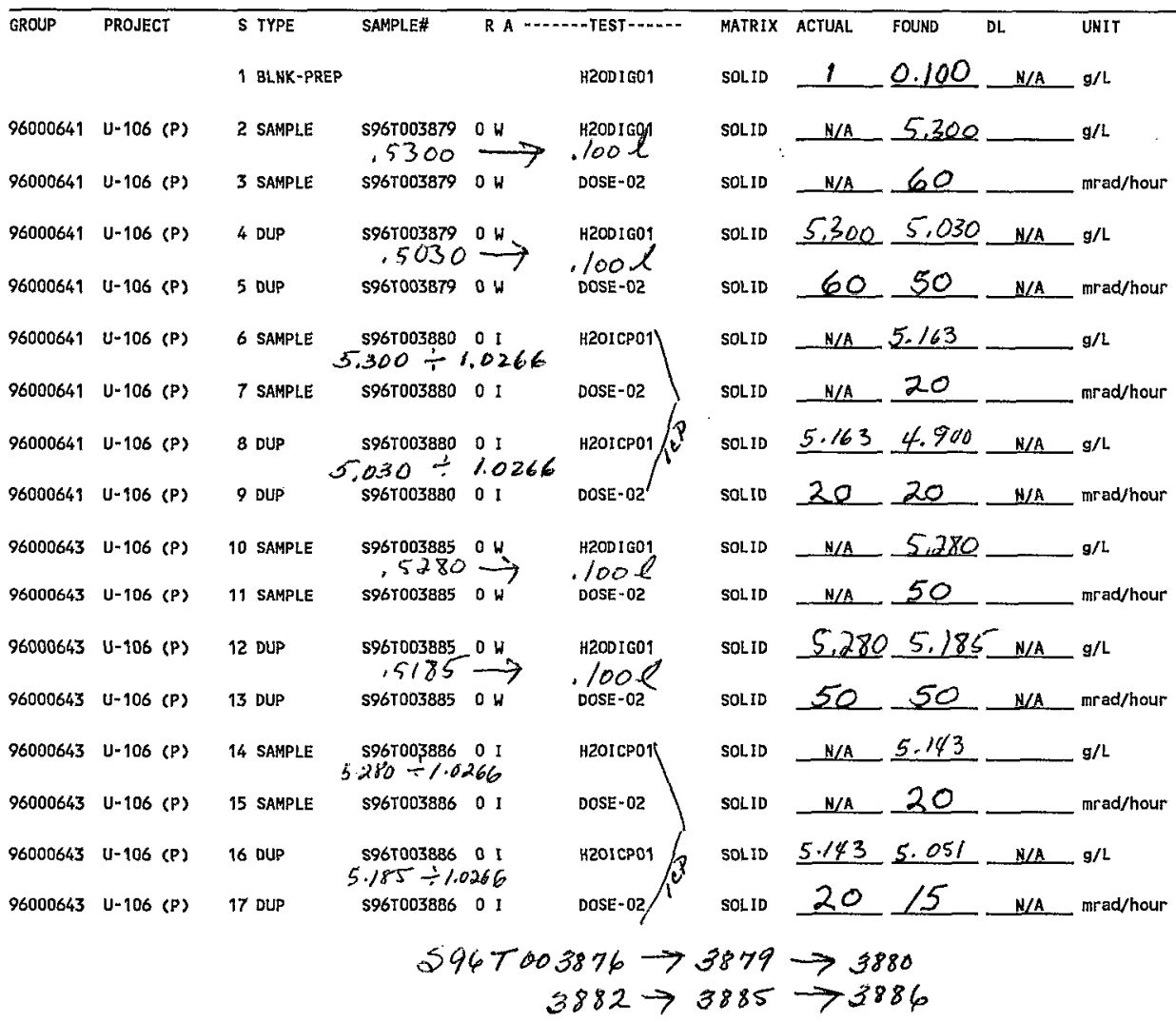

Data Entry Comments:

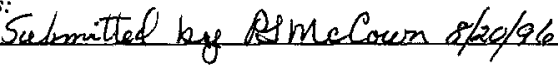

Units shown for $Q C$ (SPK \& STD) may not reflect the actual units. $D L=$ Detection Limit, $S=$ Worklist Slot Number,

$R=$ Replicate Number, $A=$ Aliquot Code. 


\section{LABCORE Data Entry Template for Worklist\#}

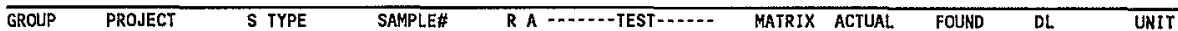

\section{Final page for worklist \#}

\section{8}

$\frac{\gamma \cot \mathbb{K}_{\mathrm{ng}}}{8 / 19 / 96}$

$\frac{7 n \text { o le }}{\text { Analyst Signature }} \quad 08-21-96$


Analyst: $\quad$ DCD $\quad$ Instrument: $A C D 01$

Book \#

Method: LA-505-159 Rev/Mod D-O

Worklist Comment: TANK\#U-106,CORE 147,SEG 2UH,3UH,3LH,4UH,4LH,5UH,5LH, ACIDIG

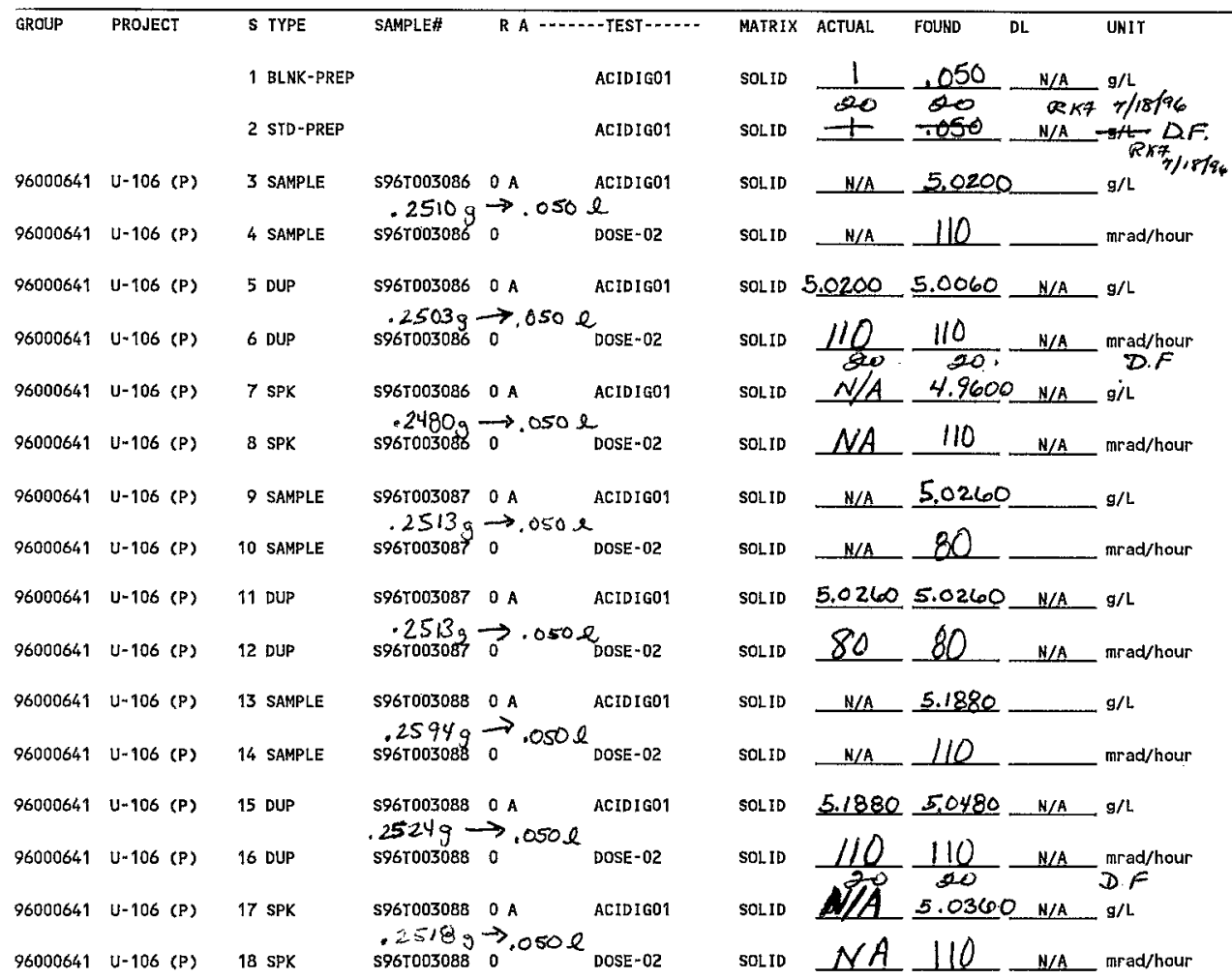

Data Entry Comments:

Units shown for $Q C$ (SPK \& STD) may not reflect the actual units. $D L=$ Detection Limit, $S=$ Worklist Slot Number, $R=$ Replicate Number, $A=$ Aliquot Code. 


\section{LABCORE Data Entry Template for Worklist\#}

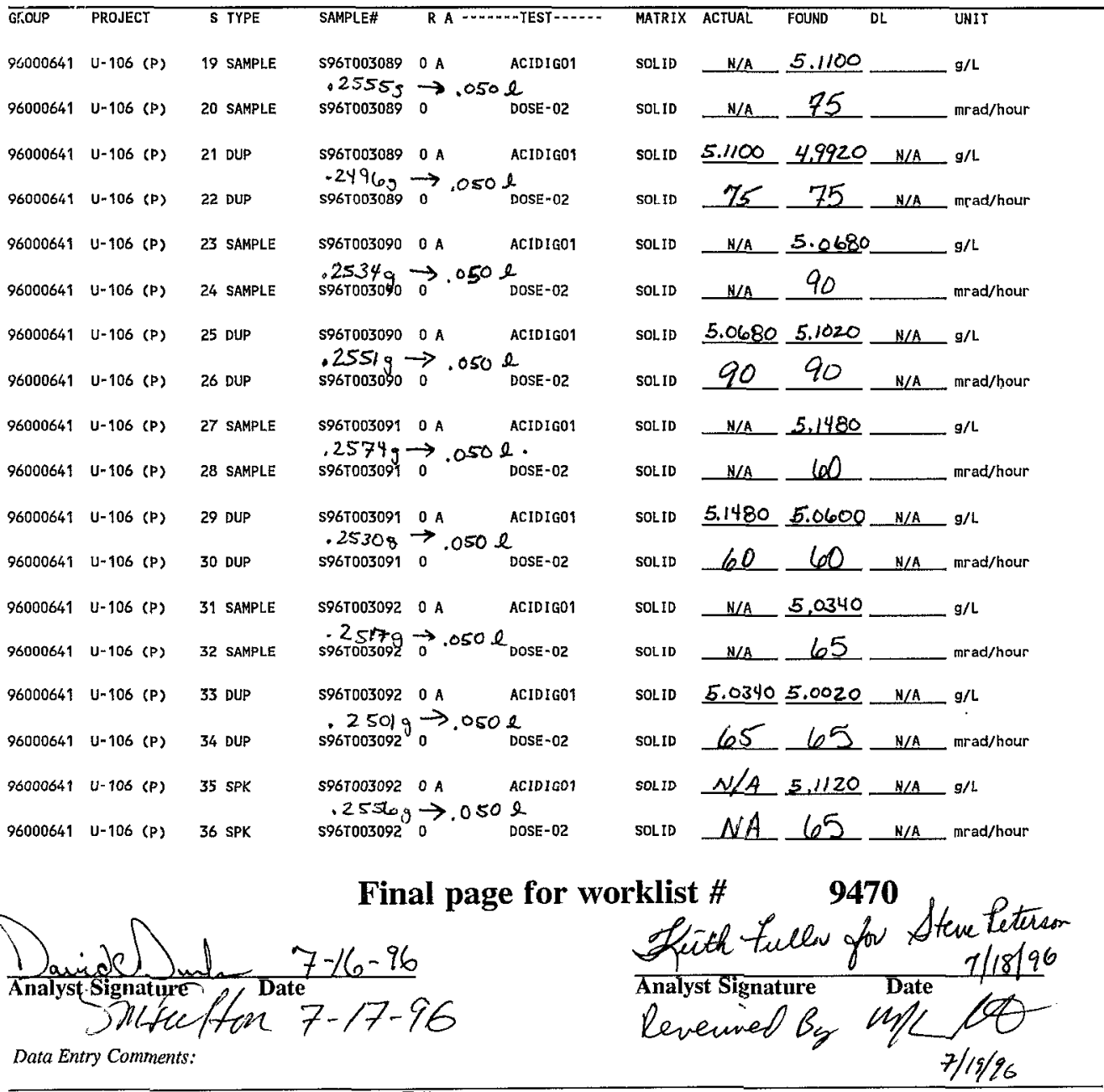

Units shown for $Q C$ (SPK \& STD) may not reflect the actual units. $D L=$ Detection Limit, $S=$ Worklist Slot Number, $R=$ Replicate Number, $A=$ Aliquot Code. 
worklistrpt Version $2.105 / 15 / 95$

WHC-SD-WM-DP-191, REV. 1

06/03/96 10:06

LABCORE Data Entry Template for Worklist\#

Page: 3

9470

$\overline{\mathrm{c} R}$

PROJECT

S TYPE

SAMPLE\#

MATRIX ACTUAl

FOUND

DE

UNIT

$$
\begin{aligned}
5947003065 & >3086 \\
3066 & \rightarrow 3087 \\
3070 & \rightarrow 3088 \\
3067 & \rightarrow 3089 \\
3068 & \rightarrow 3090 \\
3069 & \rightarrow 3091 \\
3011 & \rightarrow 309.2
\end{aligned}
$$

Note: All st da / Spikes - 20 dilution factor.

Q Pol $7 / 18 / 96$

Data Entry Comments:

GKziclen $1 / 22 / 96$

Units shown for $Q C$ (SPK \& STD) may not reflect the actual units. $D L=$ Detection Limit, $S=$ Worklist Slot Number $R=$ Replicate Number, $A=$ Aliquot Code.

167 
Method: LA-505-159 Rev/Mod

Worklist Comment: TANK\#U-106,CORE 148,SEG 2UH,2LH,3UH,3LH, ACIDIG01 RTS!

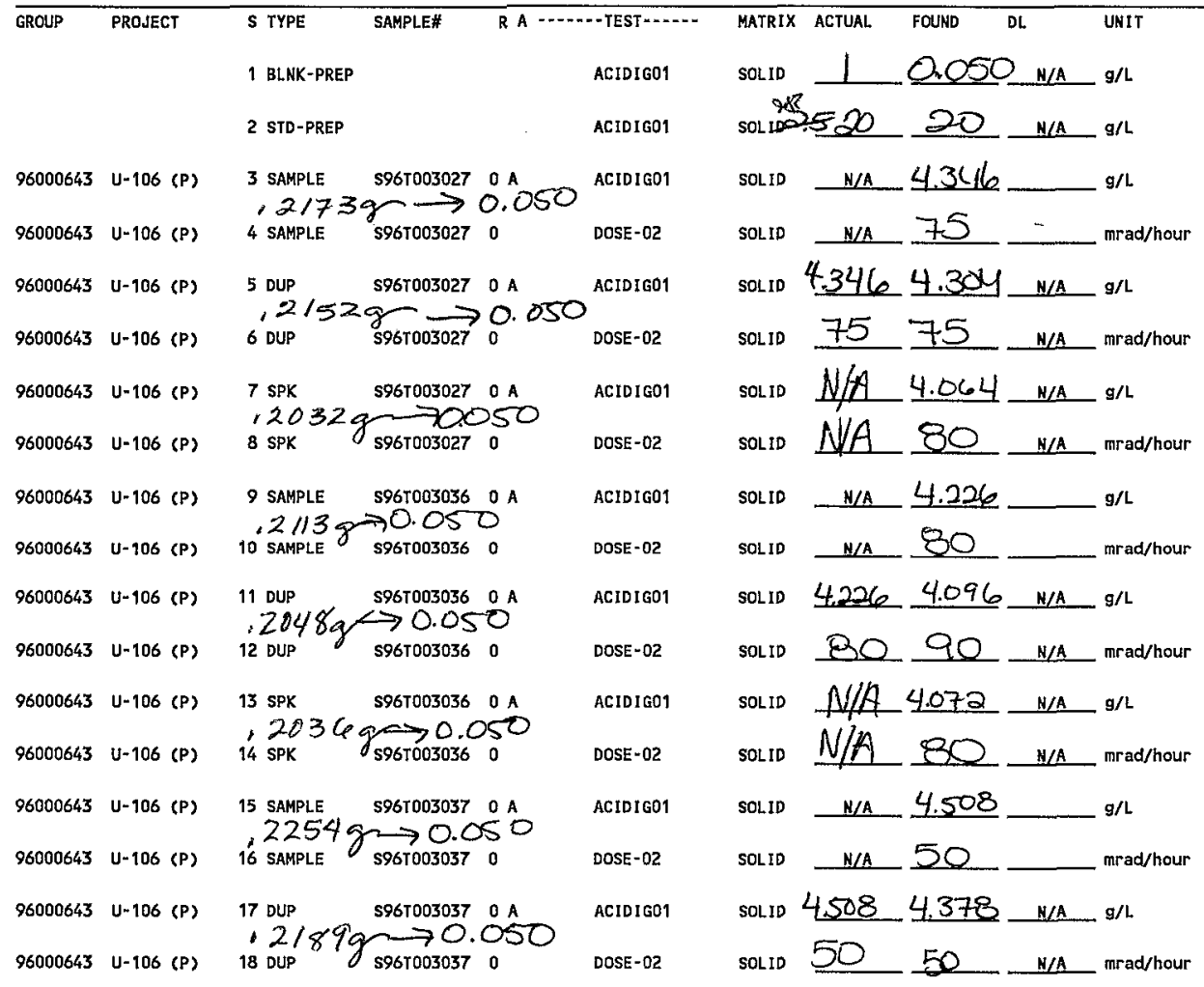

Data Entry Comments:

Units shown for QC (SPK \& STD) may not reflect the actual units. DL = Detection Limit, $S=$ Worklist Slot Number, $R=$ Replicate Number, $A=$ Aliquot Code. 
WHC-SD-WM-DP-191, REV. I

worklistrpt Version 2.1 05/15/95

Page: $\quad 2$

06/25/96 10:46

LABCORE Data Entry Template for Worklist\#

9477

GROUP PROJECT S TYPE SAMPLE\# RA $\cdots$ MATRIX ACTUAL FOUND BL

$96000643 \quad U-106$ (P)

96000643 U-106 $(P$

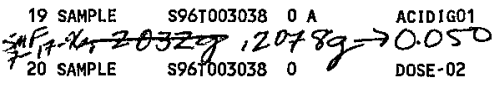

SOL. ID

SOLID
NA 4.156

UNIT

$96000643 \mathrm{U}-106(P)$

21 DUP

$596 \mathrm{~T} 003038 \quad 0 \mathrm{~A}$

ACIDIC

sol io 4.1564 .064 N/A gs

$96000643 \quad \mathrm{U}-106(P)$

22 DUP

DOSE -02

sol.10

5050

NA

mrad/hour

Final page for worklist \#

9477
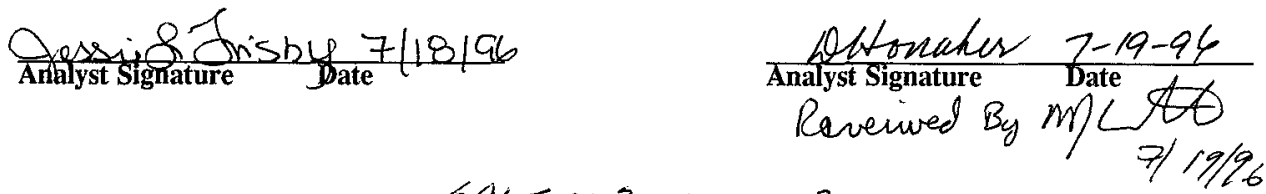

$\$ 96 T 003010 \rightarrow 3027$

$3011 \rightarrow 3034$

$3012 \rightarrow 3037$

$3013 \rightarrow 3038$

DF for all spikes is 20

Data Entry Comments:

RKzuller 1/2z/96

Units shown for $Q C$ (SPK \& STD) may not reflect the actual units. $D L=$ Detection Limit, $S=$ Worklist Slot Number, $R=$ Replicate Number, $A=$ Aliquot Code.

169 


\section{LABCORE Data Entry Template for Worklist\#}

Analyst: $\quad J 2 f$

Instrument: ACD01

Method: LA-505-159 Rev/Mod F-O

Book \#WHCLA/WHC2

Worklist Comment: TANK\#U-106,CORE 148,SEG 4UH,4LH,5UH,5LH, ACIDIG01 RTS!

\begin{tabular}{|c|c|c|c|c|c|c|c|c|c|c|c|}
\hline \multirow[t]{3}{*}{ GROUP } & \multicolumn{2}{|c|}{ PROJECT } & $S$ TYPE & SAMPLE\# & \multicolumn{2}{|c|}{ RA $\cdots \cdots$ - TEST $\cdots \cdots$} & \multirow{2}{*}{$\begin{array}{l}\text { MATRIX } \\
\text { SOLID }\end{array}$} & \multirow{2}{*}{$\begin{array}{c}\text { ACTUAL } \\
1 \\
\end{array}$} & \multirow{2}{*}{$\begin{array}{l}\text { FOUND } \\
.050 \\
\end{array}$} & \multirow{2}{*}{$\begin{array}{l}D L \\
\quad N / A \\
\end{array}$} & \multirow{3}{*}{$\begin{array}{l}\text { UNIT } \\
-g / L\end{array}$} \\
\hline & & & 1 BLNK-PREP & & & ACIDIG01 & & & & & \\
\hline & & & 2 STD-PREP & & & ACIDIG01 & SOLID & 20 & 20 & $N / A$ & \\
\hline 96000643 & $U-106$ & (P) & $\begin{array}{r}3 \text { SAMPLE } \\
.2487\end{array}$ & $\stackrel{596 T 003039}{\longrightarrow} 00$ & $50^{\circ}$ & ACIDIG01 & SOLID & N/A & 74 & & $-g / L$ \\
\hline 96000643 & U-106 & (P) & 4 SAMPLE & S96T003039 & 0 & DOSE-02 & SOLID & N/A & 100 & & - mrad/hour \\
\hline 96000643 & U-106 & (P) & $\begin{array}{l}5 \text { DUP } \\
, 2518\end{array}$ & $\stackrel{596 T 003039}{\rightarrow} 0.00$ & $\begin{array}{l}0 \mathrm{~A} \\
50\end{array}$ & ACIDIG01 & SOLID & 4.974 & 036 & N/A & g/L \\
\hline 96000643 & $v \cdot 106$ & (P) & 6 DUP & \$96T003039 & 0 & DOSE-02 & SOLID & 100 & 2 & N/A & mrad/hour \\
\hline 96000643 & $U-106$ & (P) & $\begin{array}{l}7 \text { sPk } \\
560 \mathrm{~g}\end{array}$ & $\begin{array}{l}\text { s96T003039 } \\
\rightarrow 0.05\end{array}$ & $0^{0 A}$ & ACIDIG01 & SOLID & $N \mid A$ & & N/A & $g / L$ \\
\hline 96000643 & $U-106$ & $(P)$ & $8 \mathrm{spk}$ & s96T003039 & 0 & DOSE-02 & SOLID & $1 A$ & & $N / A$ & $\mathrm{mrad} /$ hour \\
\hline 96000643 & $u-106$ & (P) & $\begin{array}{l}9 \text { SAMPLE } \\
1750\end{array}$ & $\stackrel{596 \mathrm{ro03} 040}{\rightarrow 00 \mathrm{~S}}$ & $0^{A}$ & ACIDIG01 & SOLID & & & & $g / L$ \\
\hline 96000643 & $U-906$ & $(P)^{\prime}$ & 10 SAMPEE & S96T003040 & 0 & DOSE-02 & SOLID & N/A & & & $\mathrm{mrad} / \mathrm{hour}$ \\
\hline 96000643 & $U-106$ & (P) & $\begin{array}{l}11 \text { DUP } \\
5829\end{array}$ & $\stackrel{5967003040}{\rightarrow 0.050}$ & $5^{0 A}$ & ACIDig01 & SOL ID & 4.95 & 5.164 & N/A & - $g / L$ \\
\hline 96000643 & $u-106$ & $(P)$ & 12 DUP & 596T003040 & 0 & DOSE-02 & SOLID & 90 & & N/A & $\mathrm{mrad} / \mathrm{hour}$ \\
\hline 96000643 & $U-106$ & $(P)$ & $13 \mathrm{SPK}$ & s96T003040 & $\mathrm{OA}^{\mathrm{A}}$ & ACIDIG01 & SOLID & NIA & 966 & N/A & $g / L$ \\
\hline 96000643 & $\mathrm{U}-106$ & $(P)^{\prime}$ & $14 \mathrm{SPK}$ & S96T003040 & 0 & DOSE-02 & SOLID & $n / A$ & 100 & N/A & $\mathrm{mrad} /$ hour \\
\hline 96000643 & U-106 & $\stackrel{(P)}{\theta}$ & $\begin{array}{l}15 \text { SAMPLE } \\
2099\end{array}$ & $\begin{array}{l}5967003041 \\
7 \% / 15 / 860 .\end{array}$ & $\begin{array}{l}0 \mathrm{~A} \\
233\end{array}$ & $\begin{array}{l}\text { ACIDIG01 } \\
0.0<0\end{array}$ & SOLID & N/A & 4.67 & & $g / L$ \\
\hline 96000643 & $U-106$ & $(\mathrm{P})$ & 16 SAMPLE & S96T003041 & 0 & DOSE-02 & SOLID & N/A & & & $\mathrm{mrad} / \mathrm{hour}$ \\
\hline 96000643 & $u-106$ & (P) & $\begin{array}{l}17 \text { DUP } \\
229+9\end{array}$ & s96T003041 & $0 \mathrm{~A}$ & $\begin{array}{l}\text { ACIDIG01 } \\
050\end{array}$ & SOLID & 4.67 & & N/A & $g / L$ \\
\hline 96000643 & $U-106$ & $(P)$ & 18 DUP & \$96T003041 & & DOSE-02 & SOLID & 40 & 10 & $\mathrm{~N} / \mathrm{A}$ & mrad/hour \\
\hline
\end{tabular}

Data Entry Comments:

Units shown for $Q C$ (SPK \& STD) may not reflect the actual units. $D L=$ Detection Limit, $S=$ Worklist Slot Number, $R=$ Replicate Number, $A=$ Aliquot Code. 
WHC-SD-WM-DP-191, REV .I

worklistrpt Version $2.105 / 15 / 95$

Page: 2

06/03/96 10:15

LABCORE Data Entry Template for Worklist\#

9478

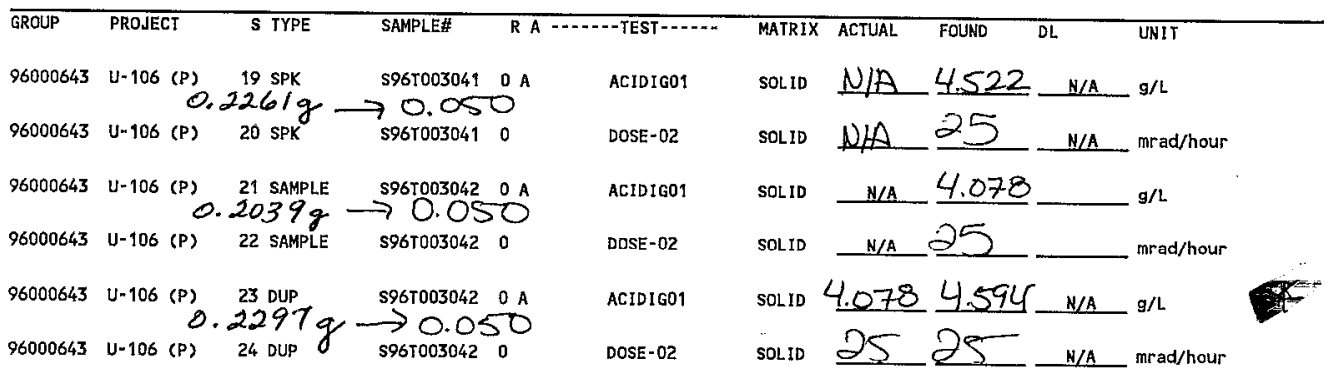

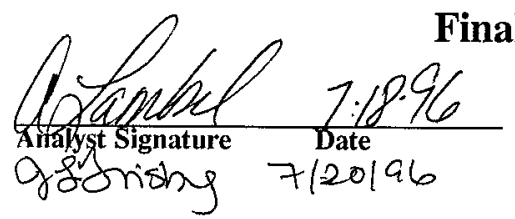

9478

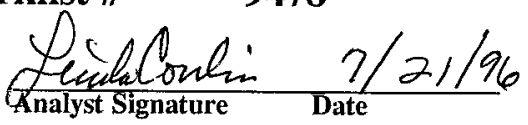

$$
\begin{aligned}
396 T 003014 & \rightarrow 3039 \\
3015 & \rightarrow 3040 \\
3016 & \rightarrow 3041 \\
3017 & \rightarrow 3041
\end{aligned}
$$

$$
\begin{array}{r}
\text { Reviewed by RKFulla } \\
7 / 22 / 96
\end{array}
$$

Data Entry Comments: Q QC FAILURED.

Units shown for $Q C$ (SPK \& STD) may not reflect the actual units. $D L=$ Detection Limit, $S=$ Worklist Slot Number, $R=$ Replicate Number, $A=$ Aliquot Code.

$\mathbb{1 7 1}$ 


\section{LABCORE Data Entry Template for Worklist\#}

Page: 1

Method: LA-505-159 Rev/Mod

Worklist Comment: U106 C147/148 COMP ACIDIG01 SKB

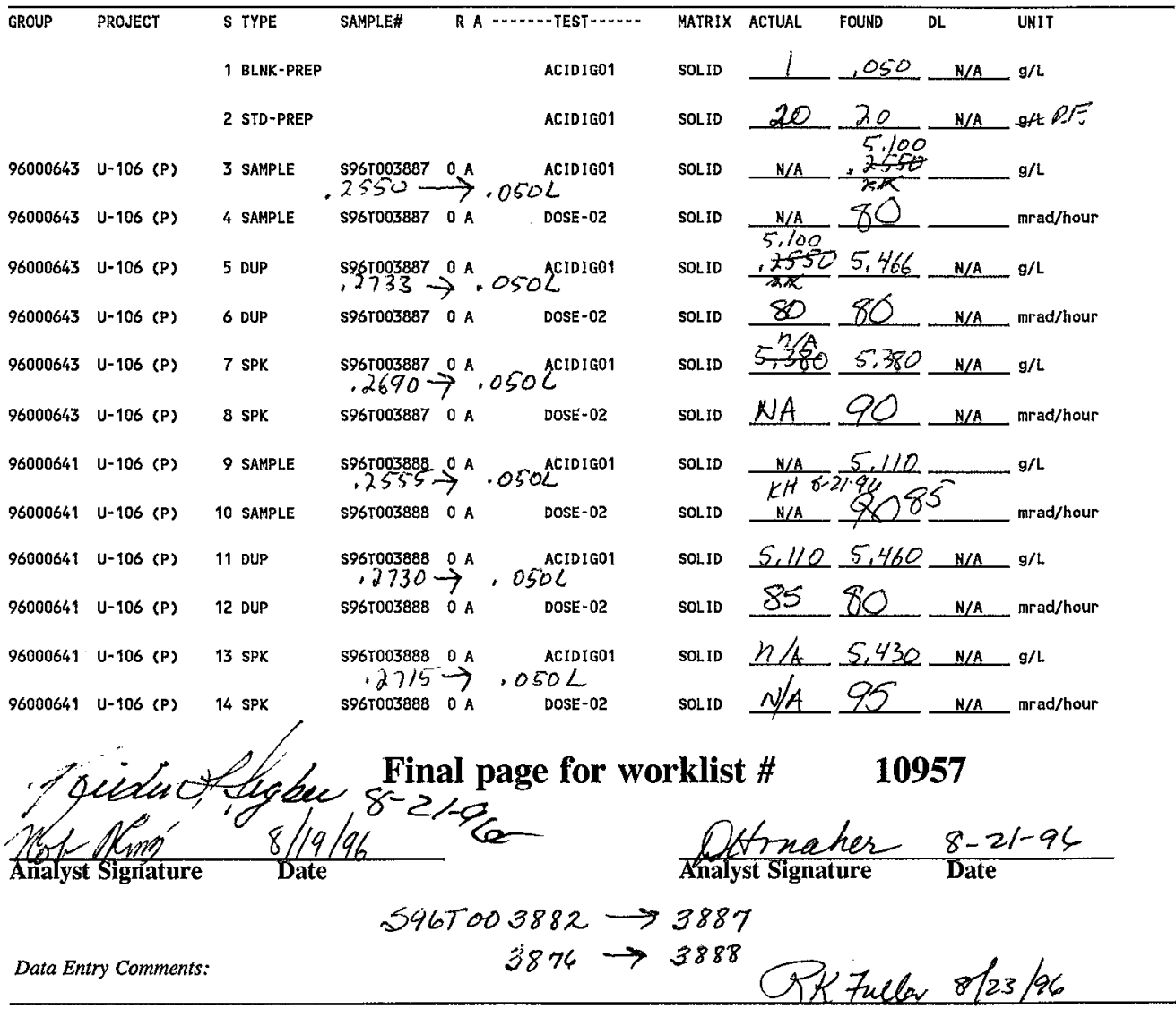

Units shown for $Q C$ (SPK \& STD) may not reflect the actual units. $D L=$ Detection Limit, $S=$ Worklist Slot Number, $R=$ Replicate Number, $A=$ Aliquot Code. 
WHC-SD-WM-DP-191, REV. 1

BULK DENSITY HORKLISTS 
WHC-SD-WM-DP-191, REV . 1

THIS PAGE WAS INTENTIONALLY LEFT BLANK 


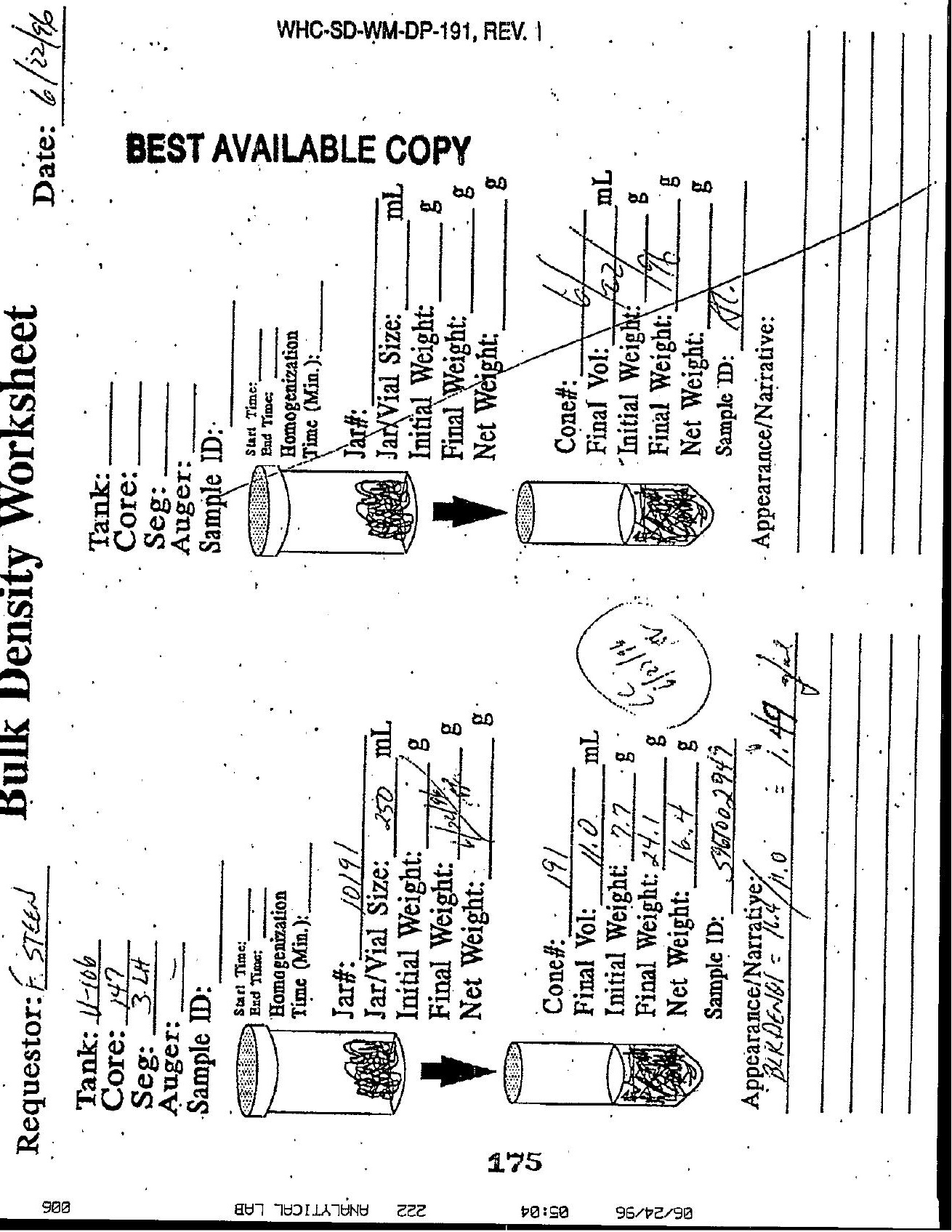




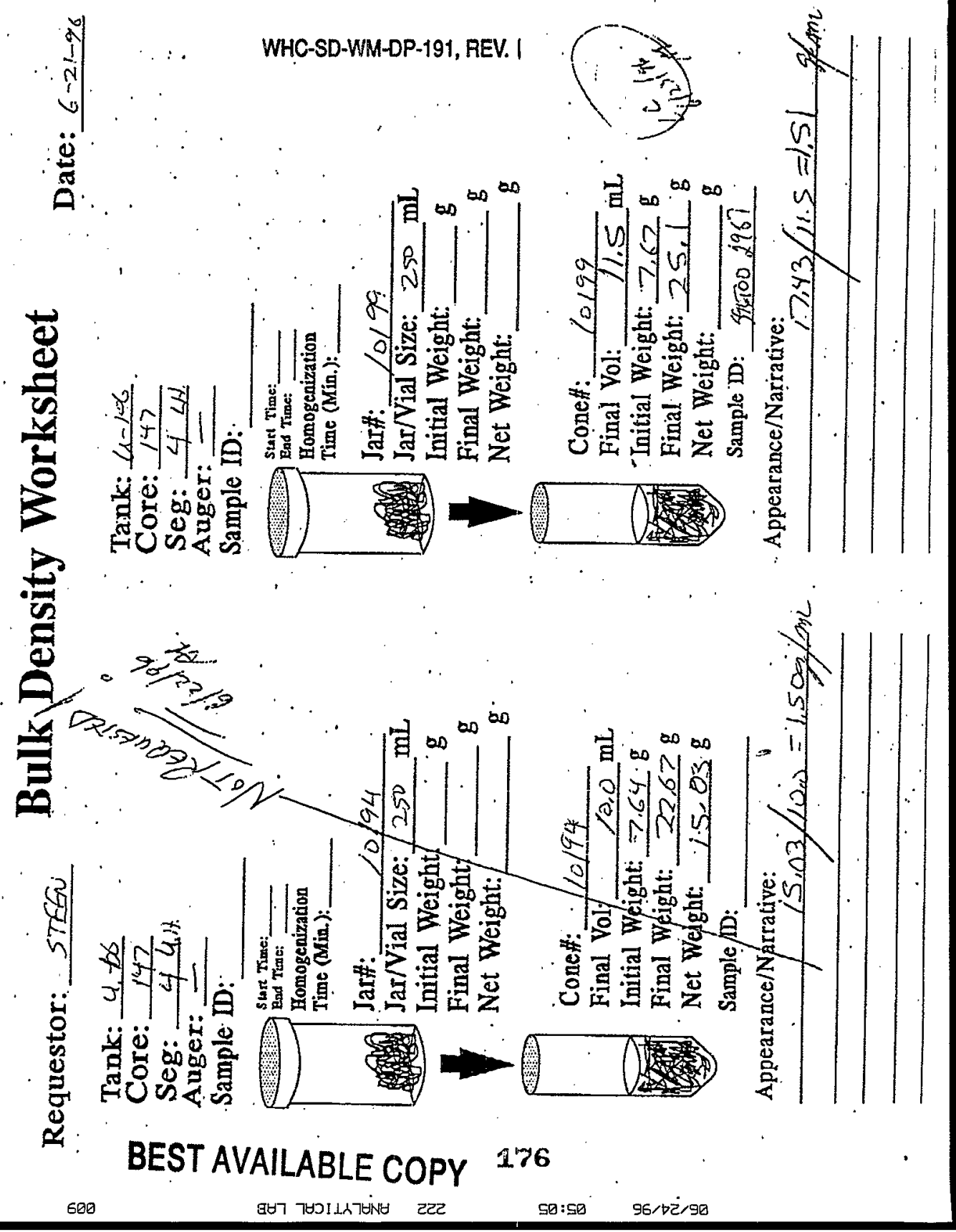




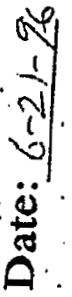

WHC-SD-WM-DP-191, REV. I
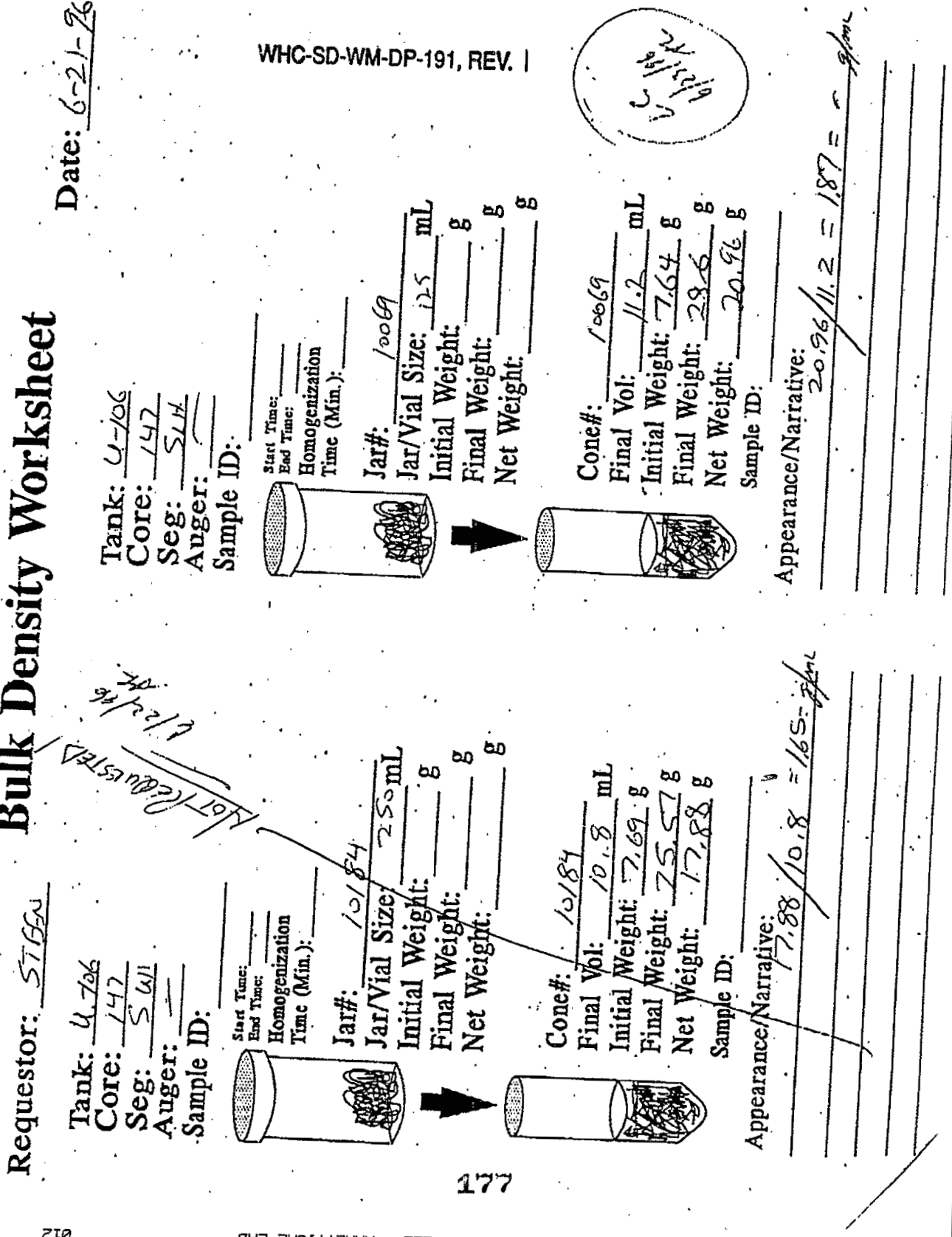<smiles>C1=C[C@H]2C[C@H]1C2</smiles>

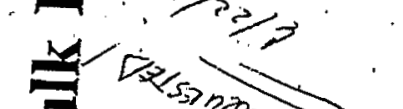

ज

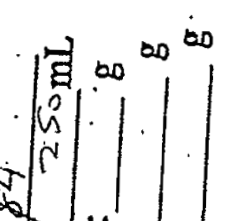

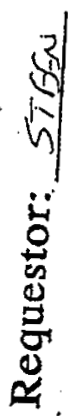


הับ

\section{WHC-SC-WM-DP-191, REV. I}
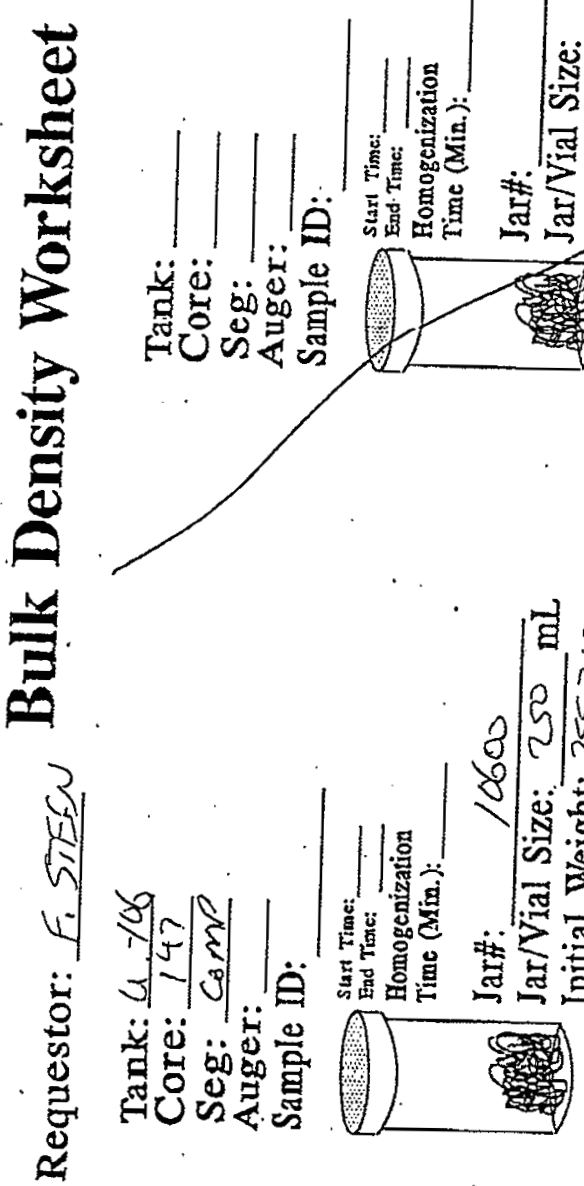

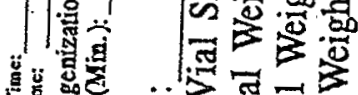

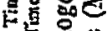

暿息是
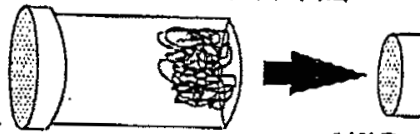

193
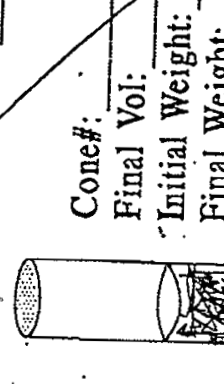

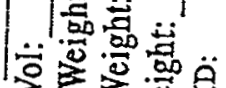

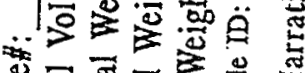

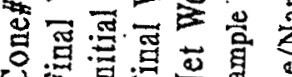

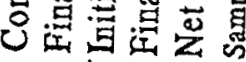
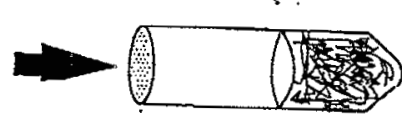

$\rightarrow$

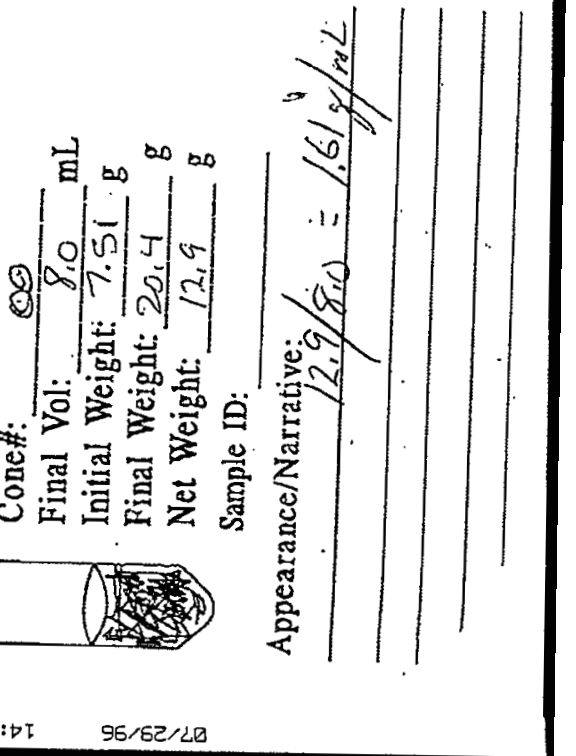




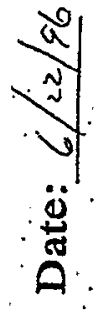

\section{WHC-SD-WM-DP-191, REV. I}
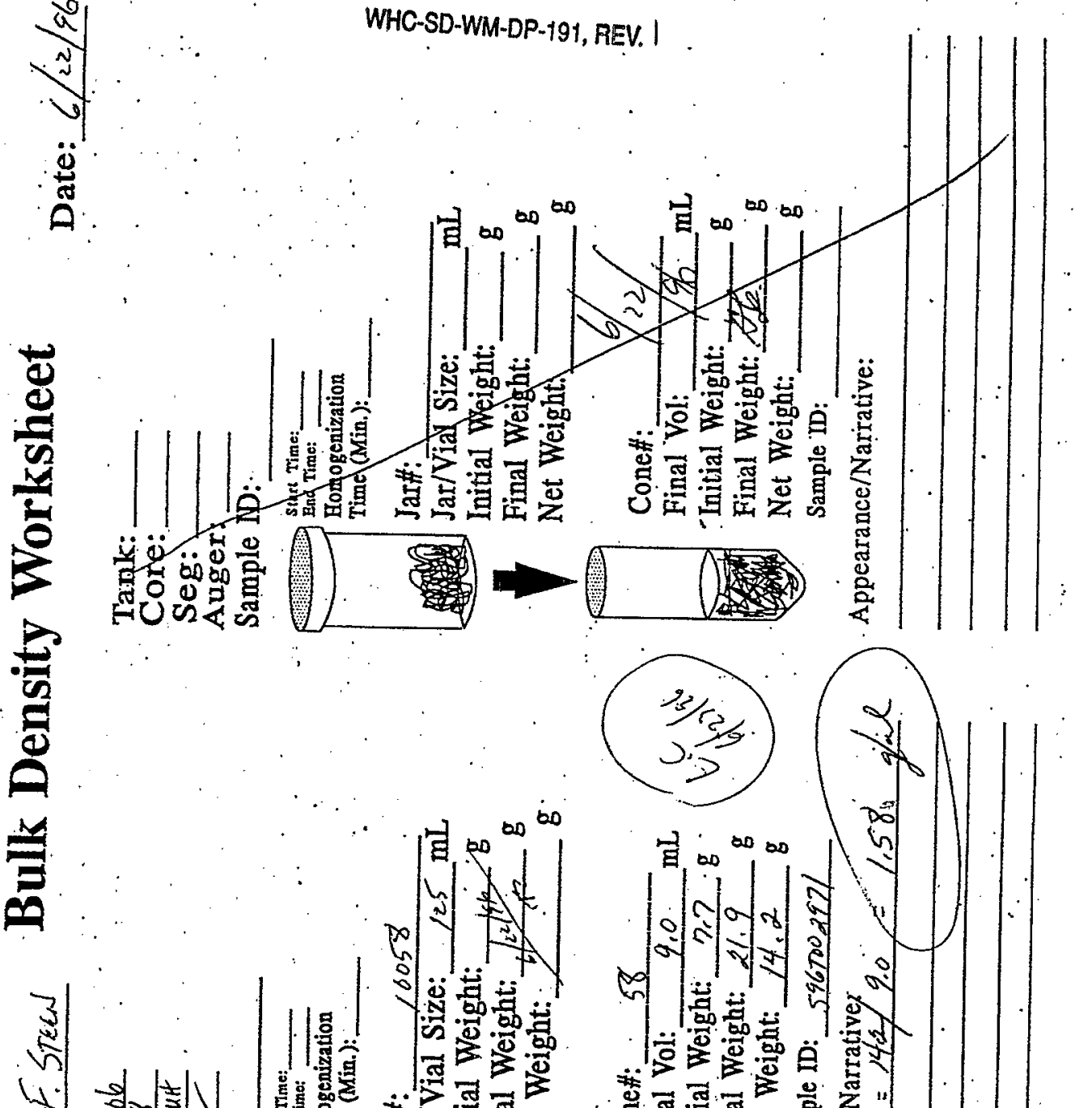

‥

त这造

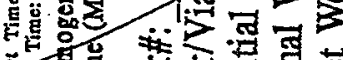

$\stackrel{*}{n}$

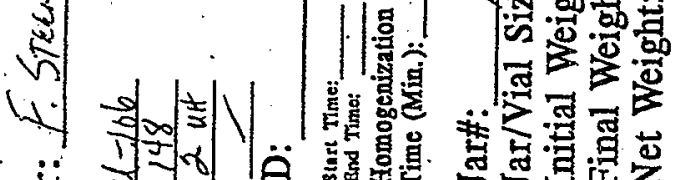

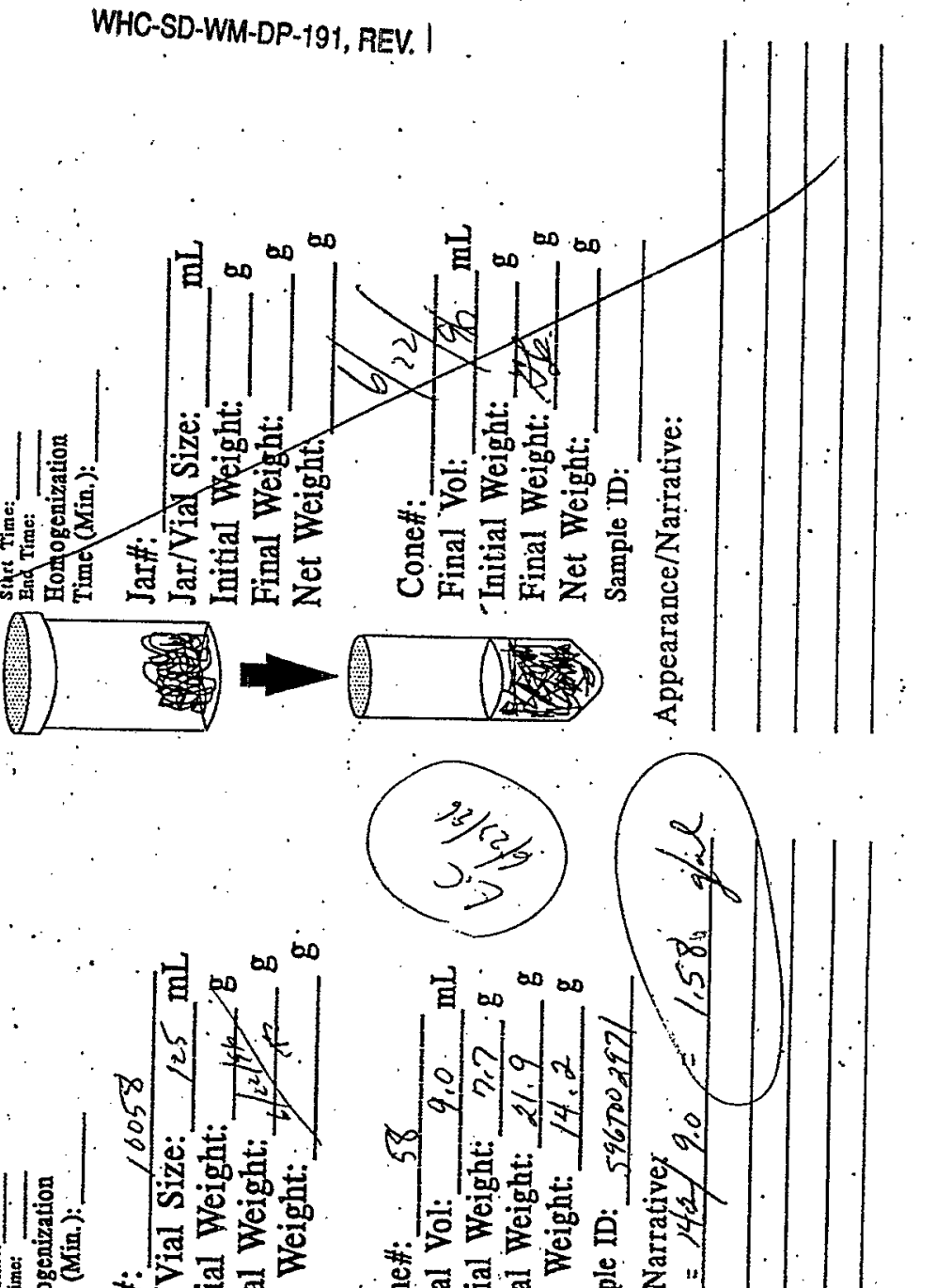

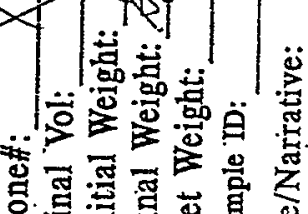

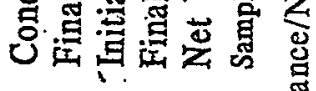

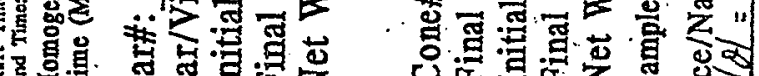

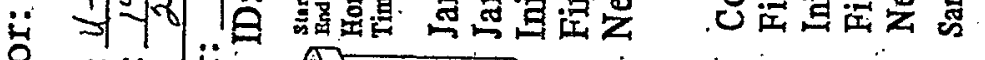

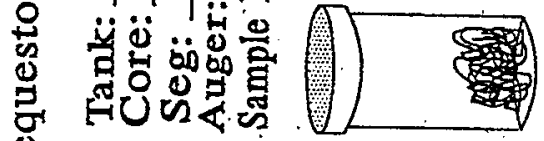
$\approx$
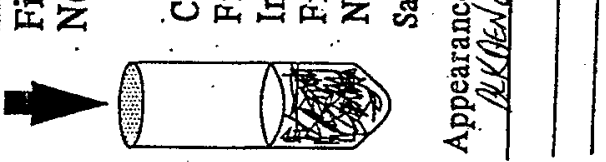


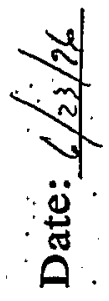

WHC-SD-WM-DP-191, REV. $I^{-}$

告

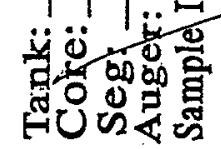

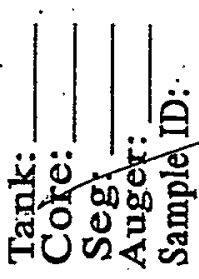

|

包 $\infty^{\infty} 0^{\circ}$ 豆

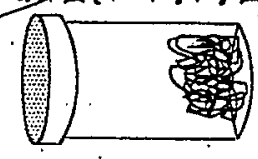

涪
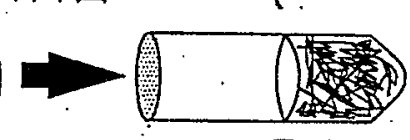

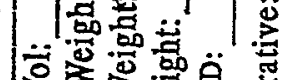

일

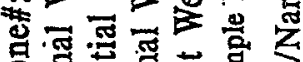

论宫重要

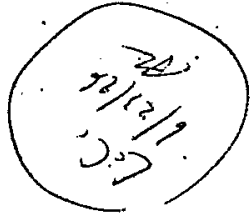

on

(1)

(1)

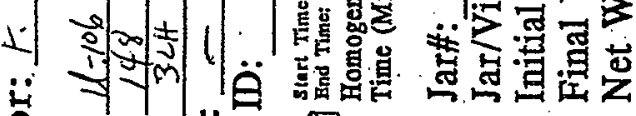

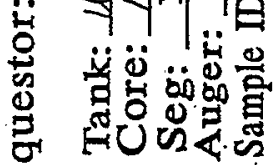
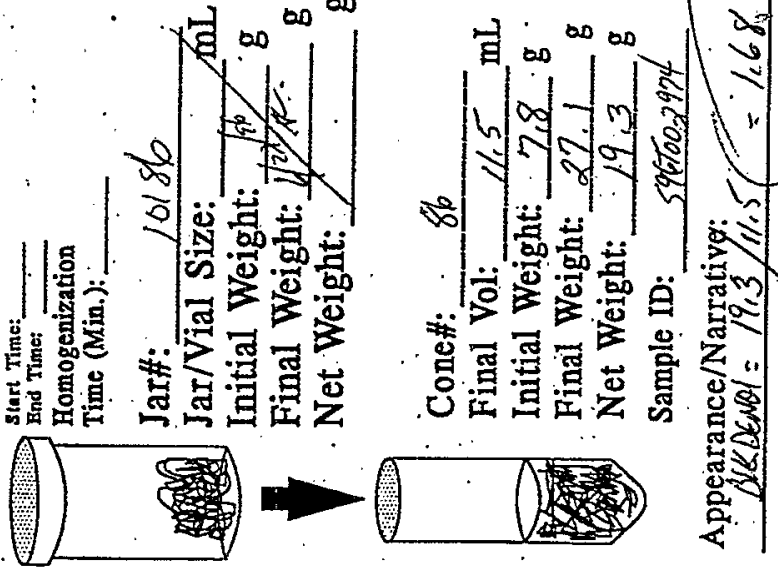


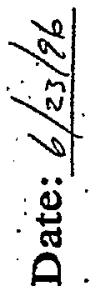

'WHC-SD-WM-DP-191, REV.I

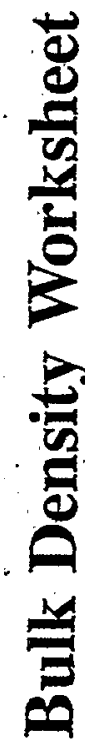

3

㤀

(4) 


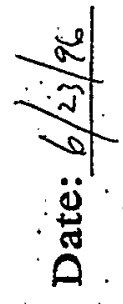

WHC-SD-WM-DP-191, REVII .

0
0
0
0
0
0
0
0
0
0
0

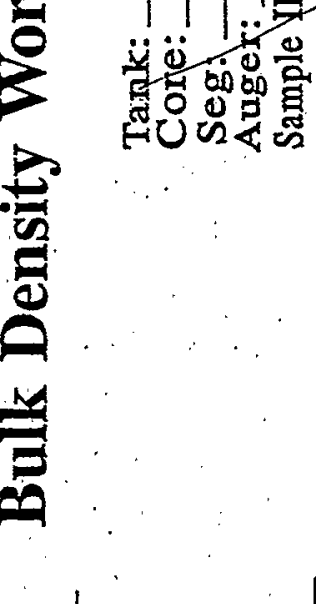

a) $\infty$
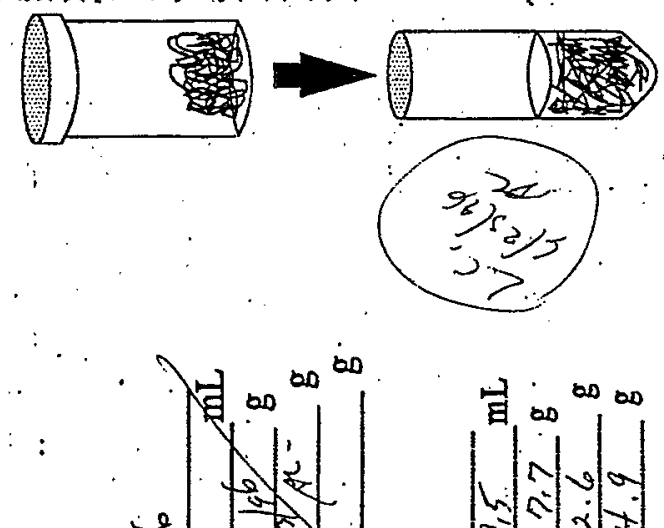

川量

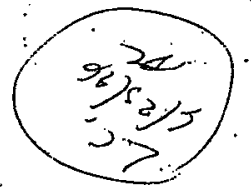

先|

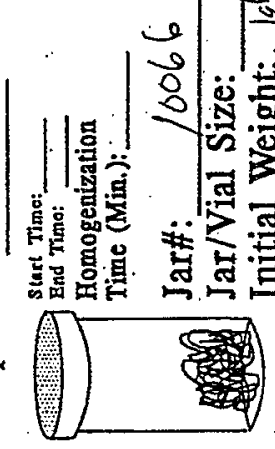

百

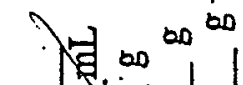

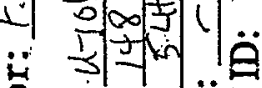

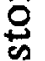

0

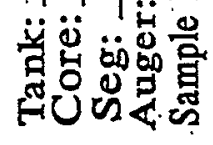

2 


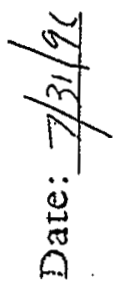

WHC-SD-WM-DP-191, REV. I
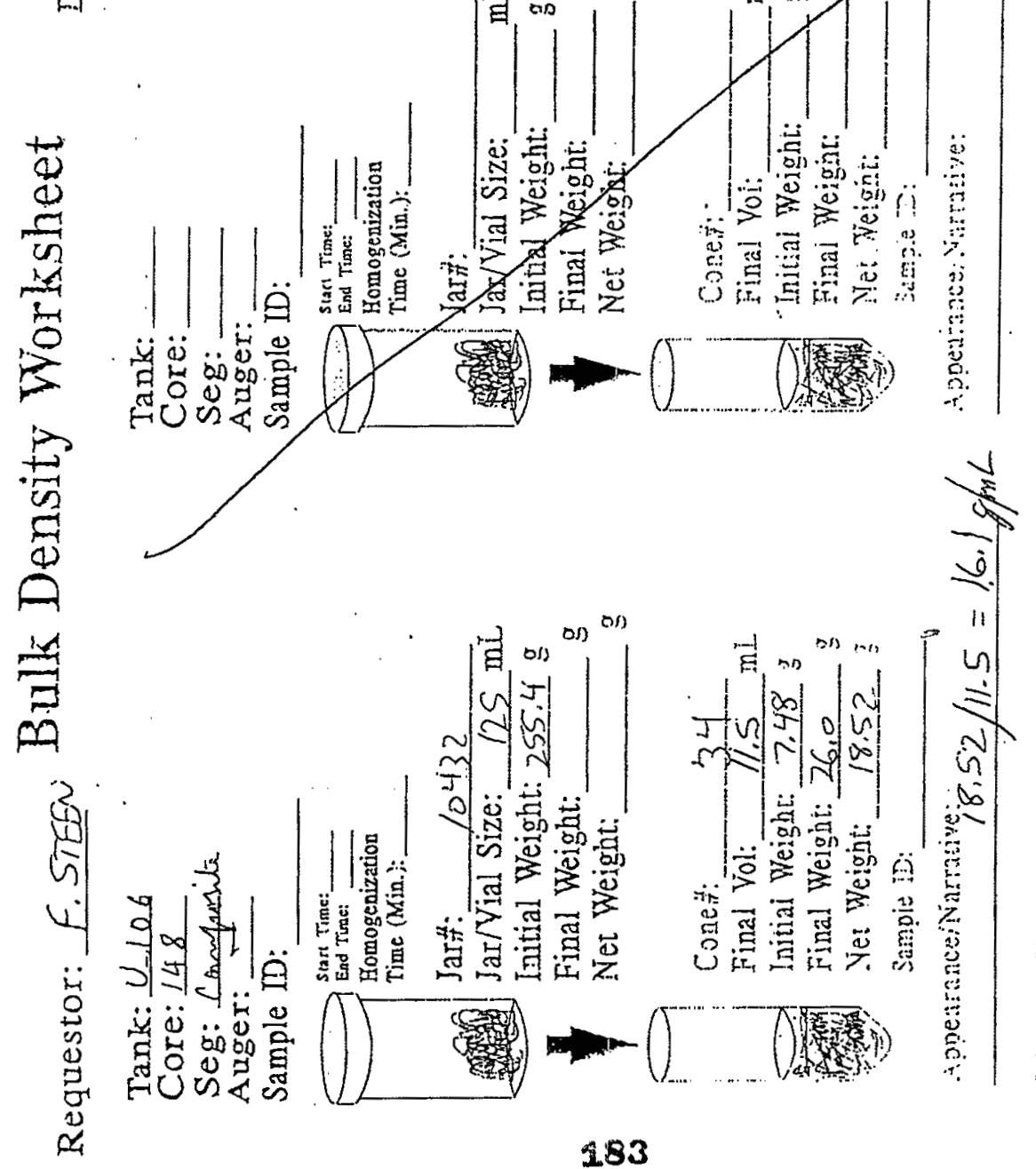


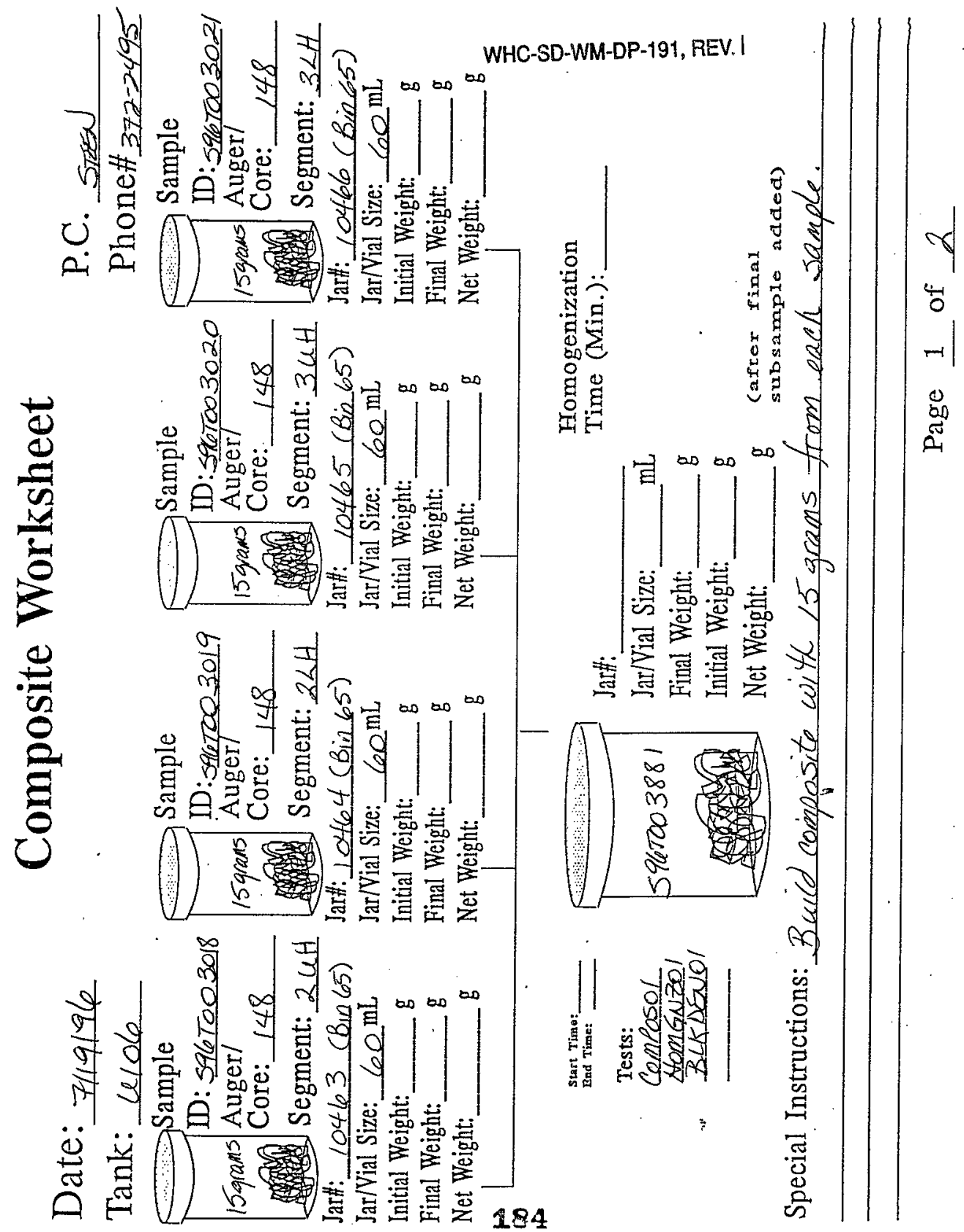




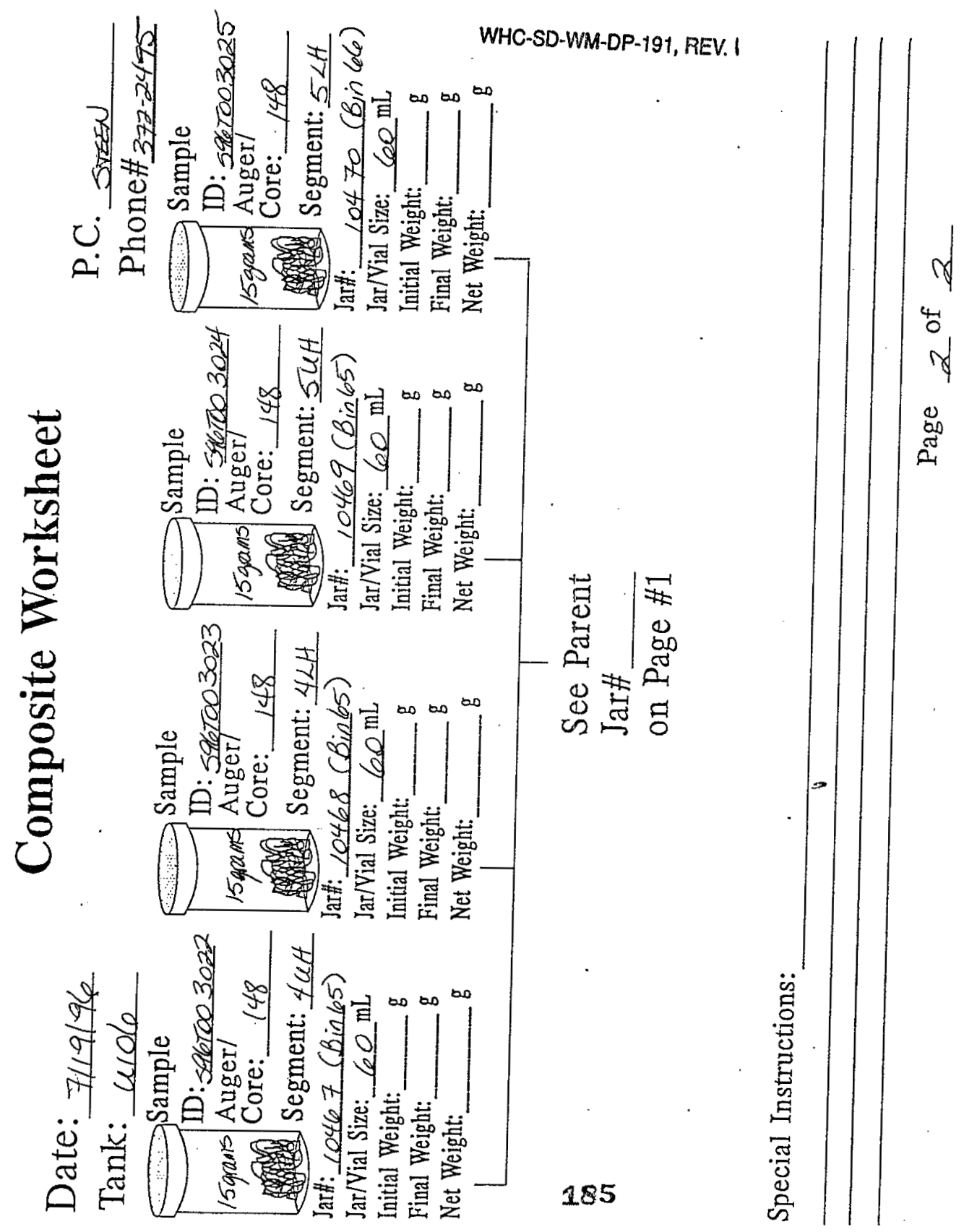




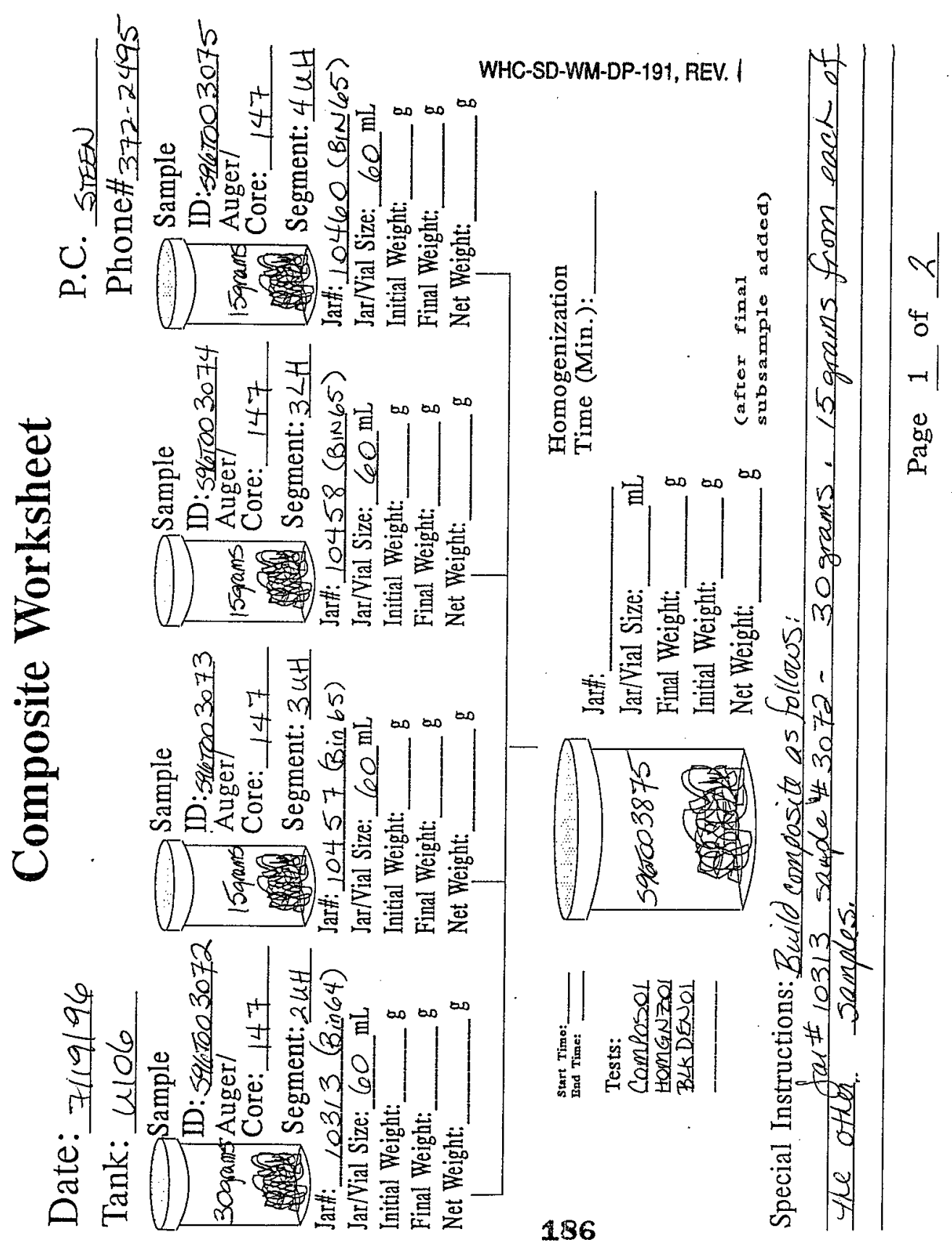




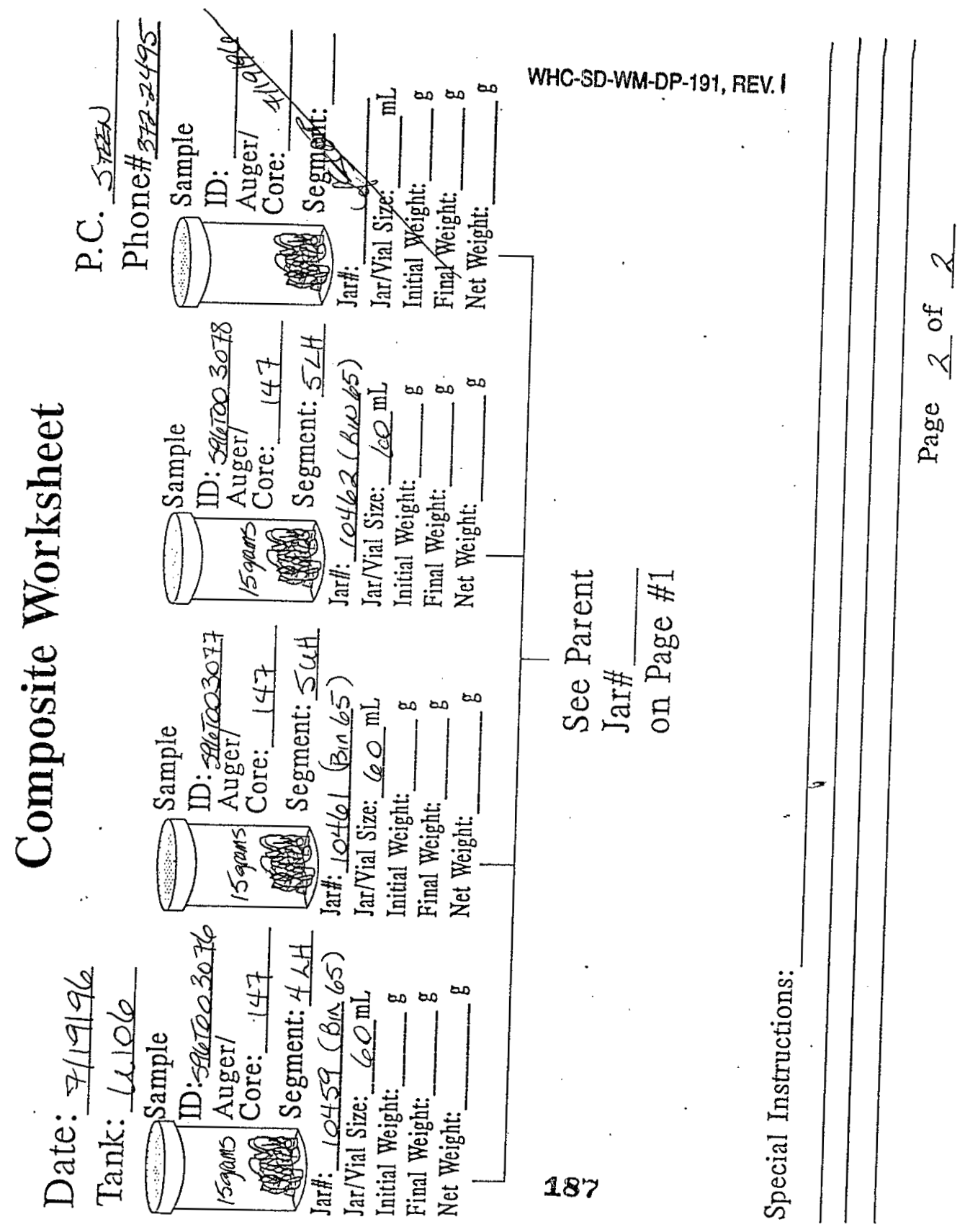


Data Reprocessed On 08/26/1996 14:57:43

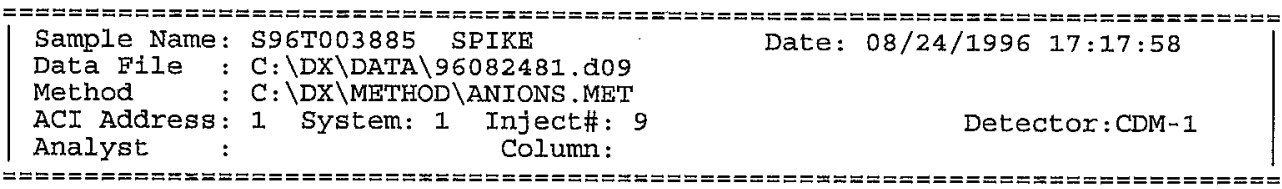

Calibration Volume Dilution Points Rate start stop Area Reject

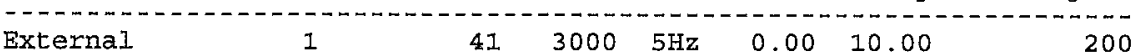

$* * * * * * * * * * * * * * * * * * * * * * * * * * *$ Peak Report: AII Peaks $* * * * * * * * * * * * * * * * * * * * * * * * * * * *$

Pk. Ret Component Concentration Height Area Bl. $\frac{9}{8}$ Delta

Num Time Name ug/ml Code

$\begin{array}{rrlrrrr}1 & 0.87 & 0.000 & 1752 & 2 \\ 2 & 1.06 \text { FLUORIDE } & 28.509 & 2640 & 13636 & 2 & 1.60 \\ 3 & 1.18 & 0.000 & 1038 & 6028 & 2 & \\ 4 & 1.65 \text { CHLORIDE } & 59.253 & 3966 & 19432 & 2 & 0.81 \\ 5 & 2.00 \text { NITRITE } & 435.281 & 18141 & 102395 & 2 & 0.00 \\ 6 & 3.06 \text { BROMIDE } & 234.781 & 5458 & 35204 & 2 & 0.11 \\ 7 & 3.40 \text { NITRATE } & 1256.153 & 27306 & 271084 & 2 & -2.20 \\ 8 & 5.14 \text { PHOSPHATE } & 242.537 & 1987 & 23310 & 1 & 2.25 \\ 9 & 6.84 \text { SULFATE } & 294.706 & 5217 & 74251 & 1 & 2.39 \\ 10 & 9.06 \text { OXALATE } & 252.341 & 2402 & 45831 & 1 & 3.27\end{array}$

Totals $\quad 2803.562 \quad 68487 \quad 592923$

File: 96082481.d09 Sample: S96T003885 SPIKE

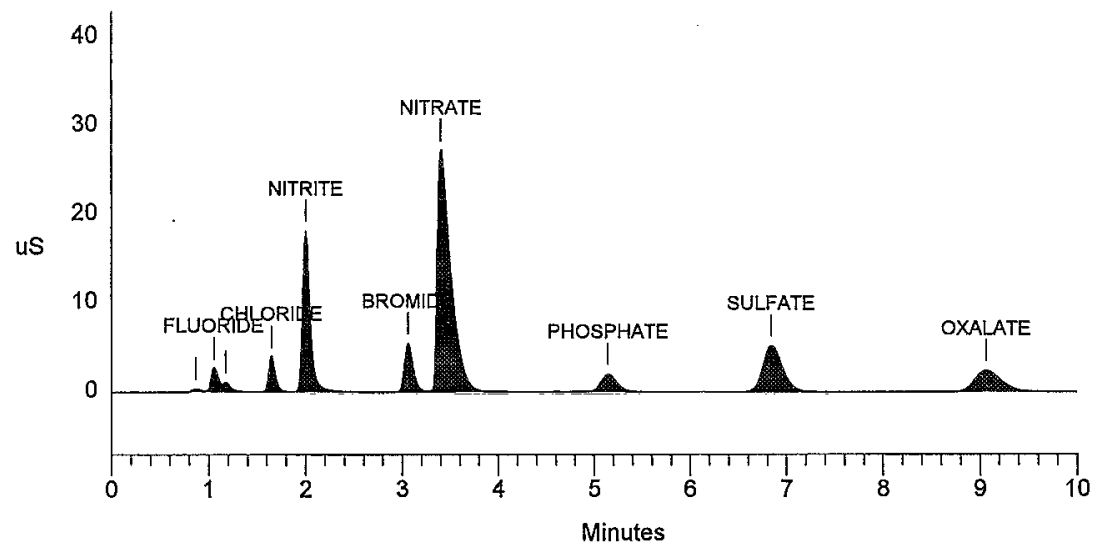


Analyst: $\quad$ EAL Instrument: IC00 ICOI Book\# $149 \mathrm{NI} . \mathrm{C}$

Method: LA-533-105 Rev/Mod D-1

Worklist Comment: @IC-01 FOR U-106 SAMPLES

RTS!

S Type Sample\# R A Test Matrix Group\# Profect

$1 \mathrm{CCB} \quad$ IIC-QC QC

$2 \mathrm{CCV} \quad$ @IC-QC QC

3 BLNK-PREP $\quad$ IC-01 SOIID

4 SAMPLE S96T003879 $0 \mathrm{~W}$ OIC-01 SOLID $96000641 \mathrm{U}-106$ (P) Analytes Requested: $\mathrm{NO}-02$

5 DUP S96T003879 $0 \mathrm{~W}$ WIC-0I SOLID

6 SPK S96T003879 $0 \mathrm{~W} @ I C-01$ SOLID

Final page for worklist \# 12421

$\frac{\text { Signature attached, } 9 / 1 / 96}{\text { Analyst Signature }}$

Analyst Signature Date

Data Entry Comments:

$S=$ Worklist Slot Number, $R=$ Replicate Number, $A=$ Aliquot Code. 


\section{LABCORE Completed Worklist Report for Worklist\# 12421}

Analyst: eal

Instrument: IC01

Method: $\angle A-533-105$ Rev/Mod

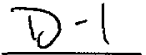

Worklist Comment: @IC-01 FOR U-106 SAMPLES

Book\# $K \mathrm{C} / \mathrm{N} / \mathrm{C}$

\begin{tabular}{|c|c|c|c|c|c|c|c|c|c|}
\hline Seq Type & Sample\# $\mathbf{F}$ & & & st & Matrix & Actual & Found & DI or Yield & Unit \\
\hline $1 \mathrm{CCB}$ & & 0 & $\operatorname{arc}-\infty$ & F & oc & 1 & $41.300-2$ & & $\mathrm{ug} / \mathrm{mL}$ \\
\hline $1 \mathrm{CCB}$ & & 0 & $\operatorname{arc}-Q \mathrm{C}$ & cr & $\mathrm{gC}$ & 1 & $<1.700-2$ & & ug/mL \\
\hline $1 \cos$ & & a & arc-oc & No: & ge & 1 & $<1.070-1$ & $\cdots \cdot$ & ug $/ \mathrm{min}$ \\
\hline $1 \mathrm{cos}$ & & 0 & QIC-QC & $\mathbf{B R}$ & $\mathbf{R C}$ & 1 & $<1.260-1$ & & $\mathrm{ug} / \mathrm{mI}$ \\
\hline $2 \operatorname{ccs}$ & $\cdots$ & 0 & aIe- $Q \mathrm{C}$ & nos & Qc & 1 & $<1.40 *-1$ & & ug/mr \\
\hline I CCB & & 0 & arc-QC & POS & $\mathrm{QC}$ & 1 & $<1.190-1$ & & $\mathrm{ug} / \mathrm{mr}$ \\
\hline $1 \mathrm{cCB}$ & & 0 & axc-ec & so4 & $\dot{\alpha}$ & $\cdots$ & $<1.360-1$ & & $\mathrm{ugg} / \mathrm{mr}$ \\
\hline $1 \mathrm{CCB}$ & & 0 & -IC-QC & OxALATE2 & $2 \mathrm{Qc}$ & 1 & $<1.05 e-1$ & & ug/mit \\
\hline $2 \operatorname{cov}$ & & 0 & -Ie-oc & $\mathbf{F}$ & Qe & 5.900 .12 & $5.860+01$ & 99.322 & \& Racovery \\
\hline $2 \mathrm{cov}$ & & 0 & arc-QC & ax & Qe & 7.90001 & $7.640+01$ & 96.709 & * Recovery \\
\hline $2 \mathrm{ccv}$ & & 0 & arc-oe & wo2 & os & 5.42002 & $5.36 n+02$ & $9 a+893$ & 4 Recovery \\
\hline $2 \mathrm{ccV}$ & & 0 & EIC-QC & BR & ge & 5.89002 & $5,430+02$ & 92.190 & \& Recovory \\
\hline $2 \mathrm{ccv}$ & & D & eIC-Q & nos & oc & 5.94002 & $5.75 e+02$ & 96.801 & \& Recorrexy \\
\hline $2 \mathrm{ccv}$ & & 0 & axc-Qce & PO4 & Qc & 5.45002 & $5.07 a+02$ & 93.028 & \& Recovery \\
\hline $2 \mathrm{cov}$ & & o & $12 C-Q C$ & so4 & Q0 & 6.31002 & $5.98 a+02$ & 94.770 & \& Recovery \\
\hline $2 \mathrm{ccv}$ & & 0 & DIC-QC & OXALATEL & $22 c$ & 5.25002 & $5.010+02$ & 95.429 & * Recovary \\
\hline 3 BLNK-PREP & & 0 & aIc-01 & $y-02$ & SOETD & 2 & $<1.30-2$ & & $\mathbf{u g} / \mathrm{g}$ \\
\hline 3 BLHK-FREP & & 0 & AIC-01 & cxi-02 & SOLID & 1 & $5.20 a-02$ & 0.052 & $\mathrm{ug} / \mathrm{g}$ \\
\hline - 3 ELMTK - PREP & & a & $\operatorname{arc}-01$ & NOZ -02 & sorip & $\mathbf{1}$ & $<1.07=-1$ & . & $\mathrm{ug} / \mathrm{g}$ \\
\hline 3 BIMTK-FREP & & 0 & aIC-01 & $B R-02$ & sorrib & $\mathbf{1}$ & $<1.268-1$ & & $\mathrm{ug} / \mathrm{g}$ \\
\hline 3 BLNK-PREP & & 0 & aIC- 02 & $x 03-02$ & SOLID & 1 & $2.600-02$ & 0.260 & ug/g \\
\hline 3 BLMK-PREP & & a & $\operatorname{arc-01}$ & P04-02 & SOLID & 2 & $<1.190-1$ & & $u g / g$ \\
\hline 3 ELNK-FREP & & 0 & (ic-01 & so4-02 & SOEID & 1 & $<1.36 \approx-1$ & & ug $/ g$ \\
\hline 3 BLNK-PREP & & 0 & AIC-01 & OXALATE2 & 2 SOLID & 1 & $<1.050-1$ & & $\mathrm{ng} / \mathrm{g}$ \\
\hline 4 SAMPLII & s965003879 & - & QIC-01. & $2003-02$ & sotion & ST/A & $1.842+05$ & 1082.000 & $\mathrm{ug} / \mathrm{s}$ \\
\hline 5 DIJP & s96T003879 & $0 \%$ & QIC-0I & No3-02 & SOLID & $1.84 \bullet+05$ & $2,12 e+05$ & 14.141 & PPD \\
\hline $6 \mathrm{SPK}$ & 5967003879 & a $w$ & arc-01. & No3-02 & sourid & 5.94002 & $4.65 e+02$ & 78.283 & \& Recuvaxy \\
\hline
\end{tabular}

\section{Comments Section:}

Comments for sample\# S96T003879 and test @IC-01 .

Atustrertue-to-NO3-standard-faiture:-nds ndo 9111/96

Spike is indicated to be out, but this recomeny of $78.283 \%$ is in pN LABQAP. ndo

Final page for worklist\# 12421

Analyst Signature Date

Analyst Signature

Date

Units shown for $Q C(B L K / B K G)$ may not reflect the actual units. 


\section{LABCORE Completed Worklist Report for Worklist\# 12421}

Seq Type Sample\# R A

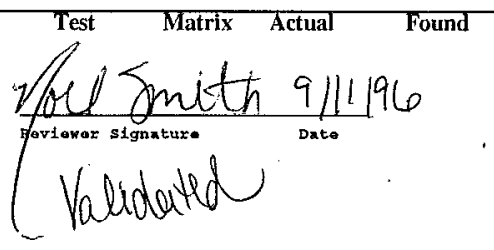




\section{LABCORE Data Entry Template for Worklist\# 12421}

Analyst: $E \mathscr{L}$ Instrument: IC00 ICOL Book\# 149N9-C

Method: LA-533-105 Rev/Mod D./

Worklist Comment: @IC-01 FOR U-106 SAMPLES

RTS!

S Type Bample\# R A

Test $\operatorname{set} 1 x$

Group\# Profect

$1 \mathrm{CCB}$

QIC-QC OC

$2 \mathrm{CCV}$

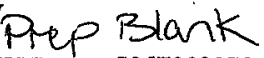

IC-QC QC

3 SAMPLE S96T003879 0 W IC-01 SOLID Analytes Requestied: NO3-02

$\begin{array}{ll}4 \text { DUP } & \text { S96T003879 } 0 \mathrm{~W} \text { OIC-01 SOLID } \\ 5 \text { SPK } & \text { S96T003879 } 0 \mathrm{~W} \text { SIC-01 SOLID }\end{array}$

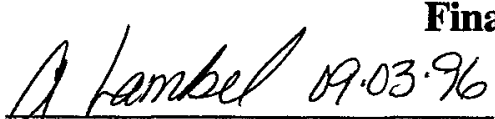

Analyst Signature Date
Analyst Signature

Date

\section{$1242 /$ sp.csv \\ Inshell $919 / 94$}

Data Entry Comments:

Batch \#910008840

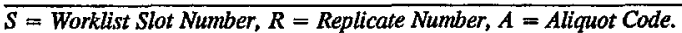


WHC-SD-WM-DP-191, REV.)

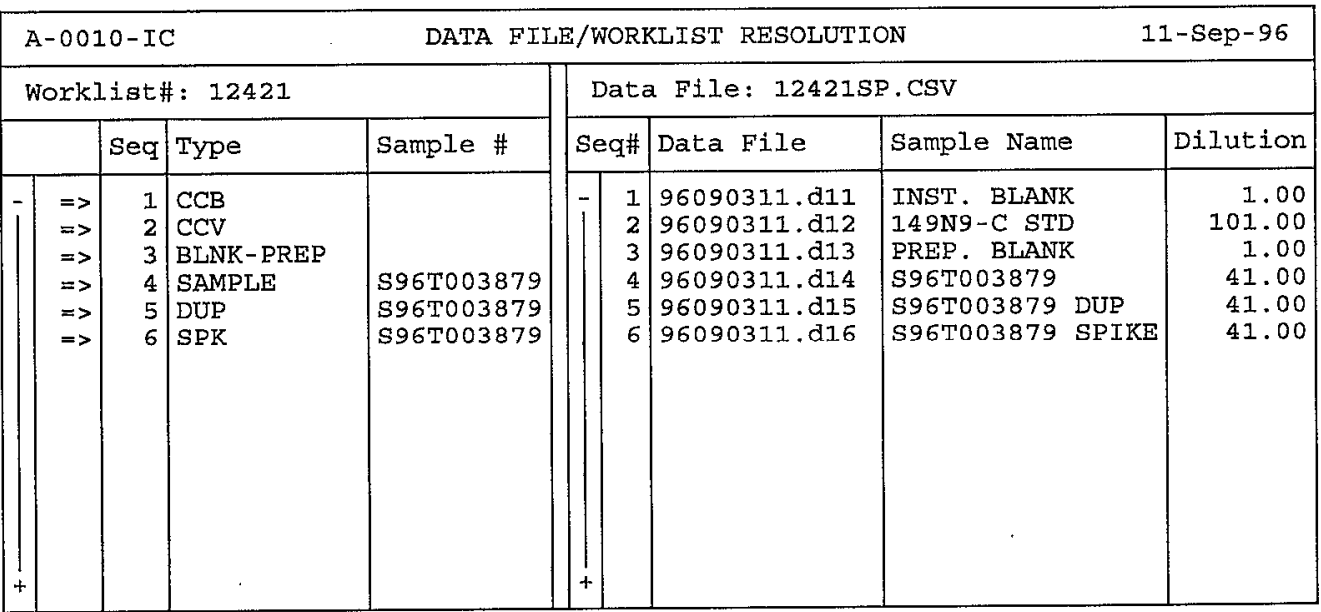

Save(F4) Abort(Shift-F3) ListFiles(Shift-F1) UploadFile(F8) 


\section{WHC-SD-WM-DP-191, REV. I}

TOC- TOTAL ORGANIC CARBON ANALYSIS REPORT

TICTOC REV 2.0

Sample: S96T03876 DUP Date: 08/31/96

Time: $16: 39: 28$

Sample size $=1 \mathrm{uL}$

Dil Factor $=1$

Blank ID \# =

Blank Value $=2.83 \mathrm{ug} /$ minute $\mathrm{C}$

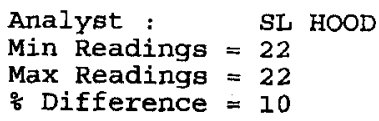

$\begin{array}{crcc}=\text { Reading }===\text { AnalysiB Time }=== & \text { Coulometer }=== & \text { Difference }== \\ 1 & 0.51 & 46.00 & 0.00 \\ 2 & 1.01 & 78.70 & 41.55 \\ 3 & 1.51 & 111.40 & 29.35 \\ 4 & 2.00 & 141.90 & 21.49 \\ 5 & 2.50 & 168.80 & 15.94 \\ 6 & 3.00 & 193.10 & 12.58 \\ 7 & 3.50 & 213.70 & 9.64 \\ 8 & 4.00 & 231.70 & 7.77 \\ 9 & 4.50 & 248.40 & 6.72 \\ 10 & 5.00 & 269.20 & 7.73 \\ 11 & 5.50 & 300.80 & 10.51 \\ 12 & 6.00 & 353.50 & 14.91 \\ 13 & 6.50 & 449.00 & 21.27 \\ 14 & 7.00 & 595.60 & 24.61 \\ 15 & 7.50 & 781.60 & 23.80 \\ 16 & 8.00 & 978.40 & 20.11 \\ 17 & 8.50 & 1169.40 & 16.33 \\ 18 & 9.00 & 1343.70 & 12.97 \\ 19 & 9.50 & 1499.30 & 10.38 \\ 20 & 10.00 & 1631.10 & 6.08 \\ 21 & 10.50 & 1748.10 & 5.53 \\ 22 & 11.00 & 1850.40 & \end{array}$

USER INPUT BLANK VALUE

BLANK VALUE $=31.13181$ micrograms carbon

BLANK FACTOR $=31.13181 / 11.00064=+2.8 \mathrm{E}+00 \quad \mathrm{ug} / \mathrm{min}$ Carbon

SAMPLE RESULTS:

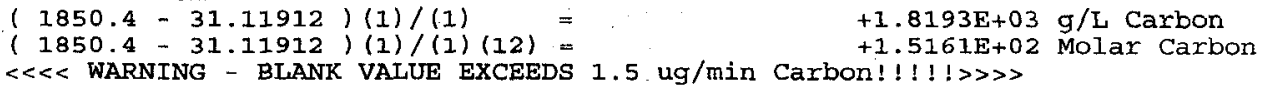

SS.086159 Sample Run By:

SL HOOD 00002 


\section{LABCORE Completed Worklist Report for Worklist\# 12677}

Analyst: dgg

Instrument: CARB2

Book\#

Method:

Rev/Mod

Worklist Comment: U-106 TICTOC. RCJ

\begin{tabular}{|c|c|c|c|c|c|c|c|c|c|}
\hline Seq Type & Sample & $\mathbf{R A}$ & & Test & Matrix & Actual & Found & DL or Yield & Unit \\
\hline 1 BLEKK & & D: & Mrtcrocl & Tre- 02 & CIOUID & 1 & $1,40 \mathrm{E} \rightarrow 0$ & 1,400 & ug/mi \\
\hline 1 BLNK & & 0 & DTICTOC1 & Toc-02 & LIQDID & 1 & $7.70 \mathrm{E}+0$ & 7,700 & $\mathbf{u g} / \mathrm{mL}$ \\
\hline $2 \mathrm{sTD}$ & & 0 & orrcrocl & Tre-02 & LTOUTD & $6.01 \mathrm{H}+02$ & $5.80 \mathrm{~m}+2$ & 97.837 & \& Reonow \\
\hline $25 T D$ & & 0 & OrIctoci & ToC-02 & LIQDID & $3.00 \mathrm{~B}+03$ & $2.65 \mathrm{E}+3$ & 88,333 & F Recove \\
\hline 3 SAMPLE & \$96T002 a60 & 0 & Orrcrocl & TIC-02 & LIOUIO & $\mathrm{N} / \mathrm{A}$ & $8.98 \mathrm{BH}+03$ & 5.000 & $\mathrm{ug} / \mathrm{mr}$ \\
\hline 3 SAMPLE & $S 96 T 002860$ & 0 & DrIctocl & Toc-02 & LIQDID & $N / A$ & $3.26 \mathrm{~K}+04$ & 40.000 & Ug/mL \\
\hline 4 DUP & 5969002860 & 0 & mricroci & TrC 02 & ITQUID & $8.98 \mathrm{~B}+3$ & $9.3 a x+3$ & 4.357 & RPD \\
\hline 4 DUP & $596 T 002860$ & 0 & ๑TICTOC1 & $\mathrm{TOC}-02$ & IIQUID & $3.26 \mathrm{~g}+4$ & $3.43 \mathrm{E}+4$ & 5.082 & RPD \\
\hline $5 \cdot \operatorname{SEK}$ & $596 T 002850$ & 0 & Mractoel & TIC- 02 & tTouto & $1000 E+02$ & $9.99 \mathrm{~K}+01$ & 99.900 & \& Recoro \\
\hline 5 SEK & $S 962002860$ & 0 & QTICTOC1 & $T \circ C-02$ & IIQTID & $1.00 \mathrm{~B}+02$ & $8.64 \mathrm{x}+01$ & 86.400 & * Recover \\
\hline 6 SAMETE & s96T0030s1 & 0 & TrTTOCl & TrC-OZ & IOOODD & $N / a$ & $9,298+03$ & 5.000 & $\mathrm{ug} / \mathrm{mt}$ \\
\hline 6 SAMPLE & S96T003051 & 0 & OTICTOCI & Toc-02 & IIQOID & $N / A$ & $3.36 \mathrm{E}+04$ & 40.000 & $\mathrm{ug} / \mathrm{mL}$ \\
\hline 7 DUTE: & $\$ 96 P 003051$ & 0 & Treroci & rue-02 & LIQUID & $9.29 \mathrm{~B}+3$ & $9,72=+3$ & 4421 & RPO \\
\hline 7 DUP & $s 962003051$ & 0 & DTICTOCI & TOC-02 & LIQTID & $3.36 \mathrm{E}+4$ & $3.35 \mathrm{E}+4$ & 0.298 & RPD \\
\hline SEK & 5962003051 & 0 & बTтCToC1 & TrC 02 & LIOTID & $1,00 \mathrm{z}+02$ & $1,15 B+02$ & 115.000 & 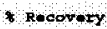 \\
\hline PK & 5967003051 & 0 & ๑TICTOC1 & Troc-02 & LIQUID & $1.00 \mathrm{~B}+02$ & $5.27 \mathrm{E}+01$ & 52.700 & ove \\
\hline
\end{tabular}

\section{Final page for worklist\# 12677}

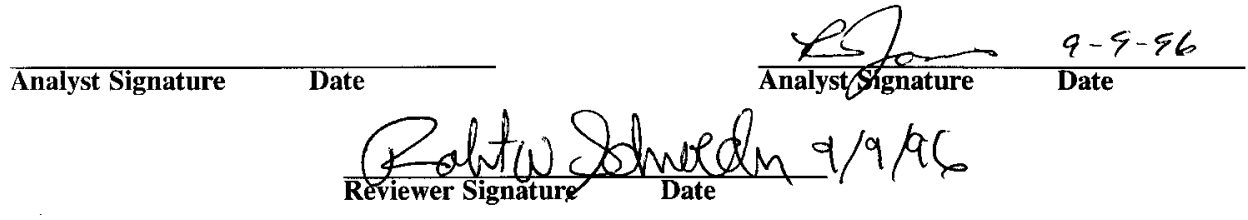

Spike for TOC S96T00305/ is reported as N/A. The spikeanallyte concentration is < than $4 x$ the sample analyte concentration. Rw Schoeder $9 / 9 / 96$

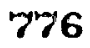




\section{LABCORE Data Entry Template for Worklist\# 12677}

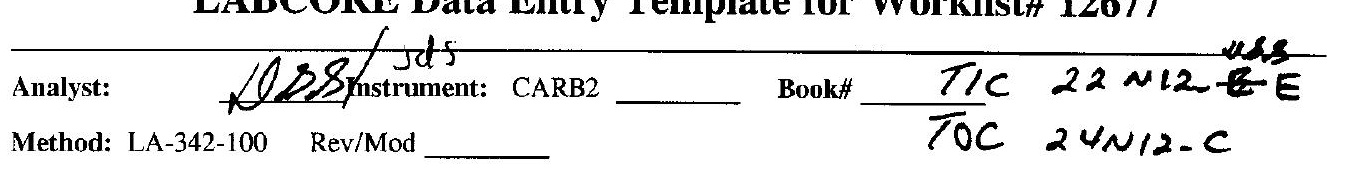

Worklist Comment: U-106 TICTOC. RCJ

S Type Sample\# R A Test Matrix Group\# Project

1 BLNK

- $2 \mathrm{STD}$

3 SAMPIE

S96 T002860 0

Analytes Requested: TIC-02, TOC-02

4 DUP

5 SPK

6 SAMPLE

7 DUP

8 SPK

@TICTOC1 LIQUID

@TICTOC1 LIQUID

@TICTOC1 LIQUID

@IICTOC1 LIQUID

@TICTOC1 LIQUID

@TICTOC1 LIQUID

S96T0030510 Analytes Requested: TIC-02, TOC-02

S96T003051 0 @TICTOC1 LIQUID

S96T003051 0

@TICTOC1 LIQUID

\section{Final page for worklist \# 12677}
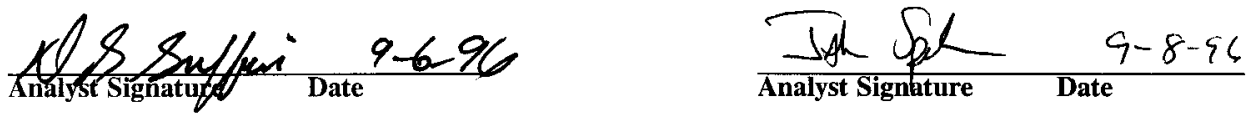

Data Entry Comments:

$S=$ Worklist Slot Number, $R=$ Replicate Number, $A=$ Aliquot Code.ryry 
WHC-SD-WM-DP-191, FEY I
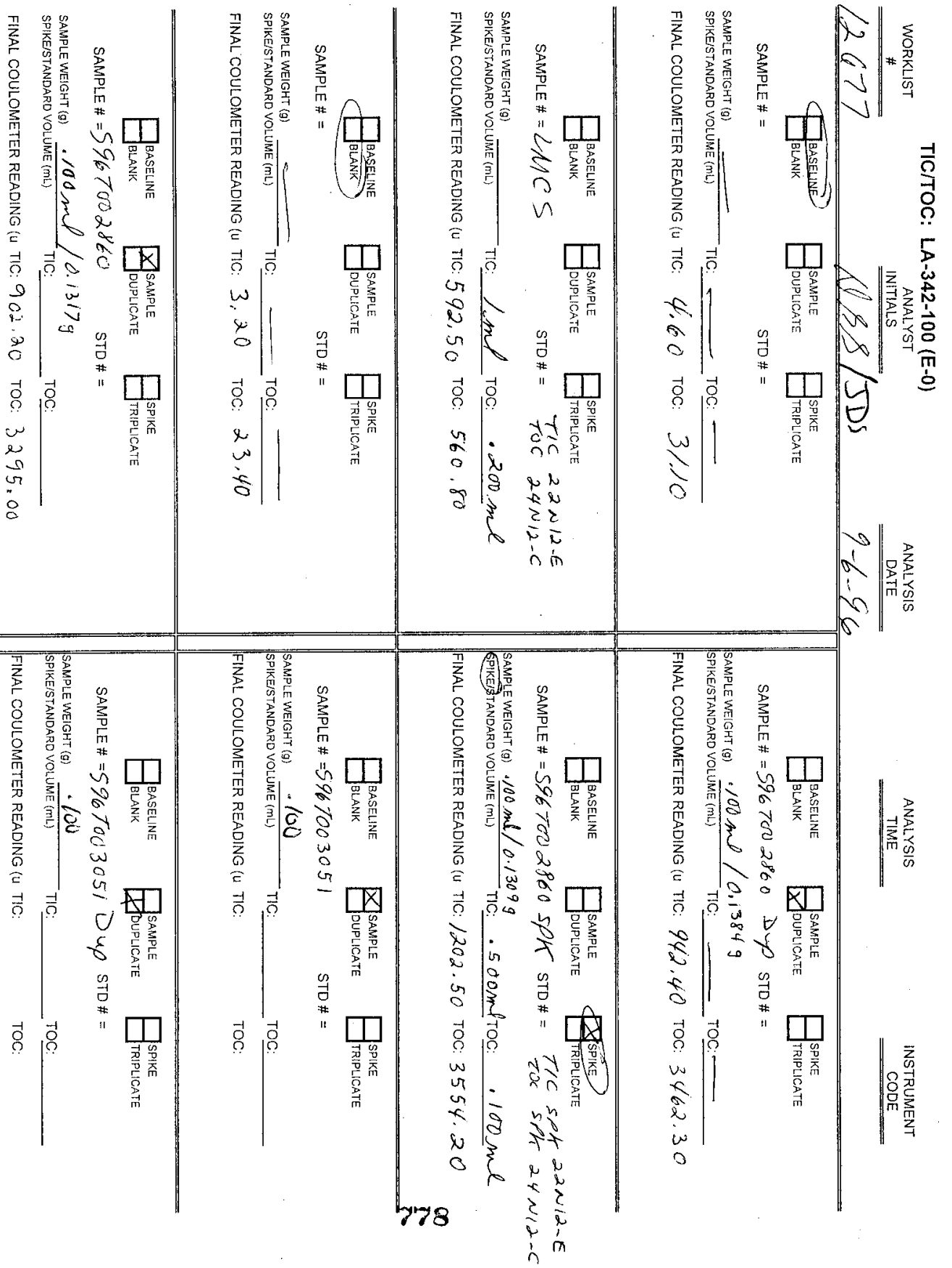


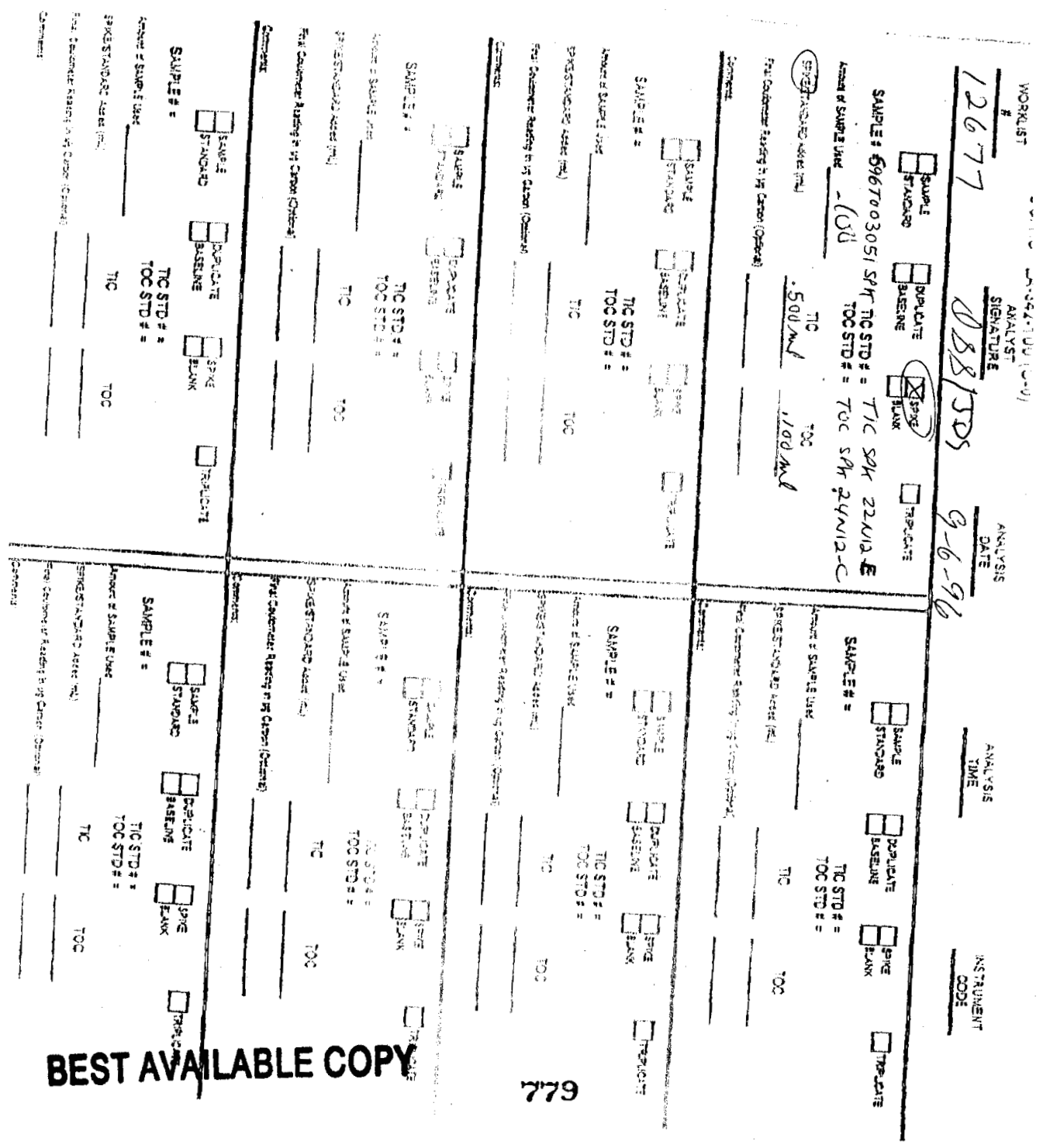


WHC-SD-WM-DP-191, FEV I

WORKBOOK PAGE: BLANK1

\begin{tabular}{|c|c|c|c|c|}
\hline TIC/TOC : LA-342 & $-100(E-0)$ & & TIC & TOC \\
\hline 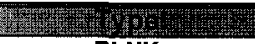 & Sample Size in $\mathrm{mL}$ & (SS) & 0.0000 & 0.0000 \\
\hline BLNK & Dilution Factor & (DF) & WIP & Ty \\
\hline 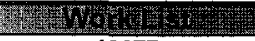 & $\mu \mathrm{g}$ of Carbon in Sample & (C1) & 3.2 & 23.4 \\
\hline 12677 & $\mu g$ of Carbon from Baseline & (C2) & 4.6 & 31.1 \\
\hline
\end{tabular}

@TICTOC1

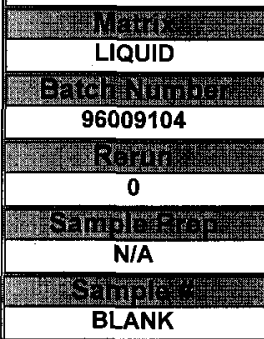

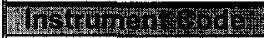

ug of Carbon $=|\mathrm{C} 1-\mathrm{C} 2|$

CARB2

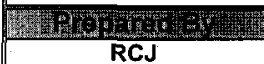

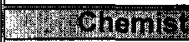

RWS

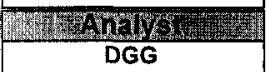

Darow

09/09/96

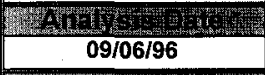

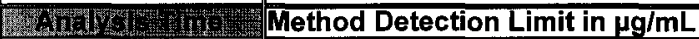

10:58 AM

4514.

U-106

\begin{tabular}{|l|r|r|}
\cline { 2 - 3 } \multicolumn{1}{c|}{} & TIC & TOC \\
\hline Method Detection Limit in $\mu \mathrm{g} / \mathrm{mL}$ & 50 & \\
\hline$\mu \mathrm{g}$ of Carbon & & $4.70 \mathrm{E}+00$ \\
\hline
\end{tabular}


WHC-SD-WM-DP-191, REV. I

WORKBOOK PAGE: STD2

TIC/TOC : LA-342-100 (E-0)

LIQUIDS

\begin{tabular}{|c|c|c|c|c|}
\hline IC/TOC & LIQU & & TIC & TOC \\
\hline W & Sample Size in $\mathrm{mL}$ & (SS) & 1.0000 & 0.2000 \\
\hline STD & Dilution Factor & (DF) & 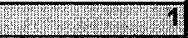 & Why \\
\hline 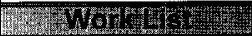 & Final Coulometer Reading in $\mu \mathrm{g}$ & (C1) & 592.5 & 560.8 \\
\hline 12677 & $\mu g$ of Carbon from Baseline & (C2) & 46 & 31.11 \\
\hline 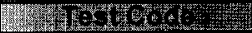 & Standard Book Number & & 22N12E & 24N12C \\
\hline @TICTOC1 & Standard Value $(\mu \mathrm{g} / \mathrm{ml})$ & & 601 & 3001 \\
\hline
\end{tabular}

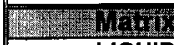

LIQUID

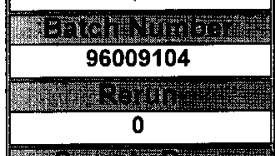

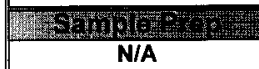

QC Actual in $\mu \mathrm{g} / \mathrm{mL}=$ Standard Value $(\mu \mathrm{g} / \mathrm{mL})$

QC Found in $\mu \mathrm{g} / \mathrm{mL}=\left(C 1-C_{2}\right) * D F / S S$

QC Found in $\mu \mathrm{g} / \mathrm{mL}$ for $\mathrm{TIC}=5$ if $\mathrm{C} 1<\mathrm{C} 2$

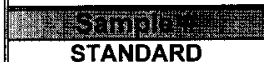

QC Found in $\mu \mathrm{g} / \mathrm{mL}$ for $\mathrm{TOC}=\mathbf{4 0}$ if $\mathrm{C} 1<\mathrm{C2}$

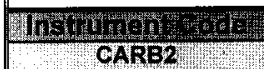

$\%$ Recovery $=$ QC Found $/$ QC Actual * 100

RCJ

$\frac{1615}{\text { RWS }}$

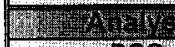

DEC

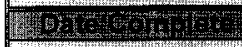

09109196

1.

09106196

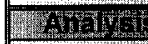

$1058 \mathrm{AWH}$

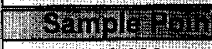

0106

\begin{tabular}{|l|r|r|} 
& \multicolumn{1}{|c|}{ TIC } & \multicolumn{1}{|c|}{ TOC } \\
\hline Method Detection Limit in $\mu \mathrm{g} / \mathrm{mL}$ & 5 & 40 \\
\hline QC Actual in $\mu \mathrm{g} / \mathrm{mL}$ & $6.01 E+02$ & $3.00 \mathrm{E}+03$ \\
\hline QC Found in $\mu \mathrm{g} / \mathrm{mL}$ & $5.88 E+02$ & $2.65 \mathrm{E}+03$ \\
\hline Percent Standard Recovery & 97.8 & 88.3 \\
\hline
\end{tabular}

Data Entered By:

Signature of Chemist:

STANDARD.WB1 REV 1.0 
WHC-SD-WM-DP-191, REV. I

\begin{tabular}{|c|c|c|c|c|}
\hline \multicolumn{3}{|c|}{ WORKBOOK PAGE: SAM3 } & & \\
\hline TIC/TOC : LA-34 & 2-100 (E-0) & & TIC & $\overline{\text { TOC }}$ \\
\hline 19. & Sample Size in $\mathrm{mL}$ & (SS) & 0.1000 & 0.1000 \\
\hline SAMPLE & Dilution Factor & (DF) & 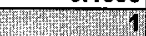 & 资 \\
\hline MOHOH & $\mu \mathrm{g}$ of Carbon in Sample & (C1) & 902.2 & 3295 \\
\hline 12677 & pg of Carbon from Baseline & (C2) & 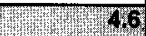 & 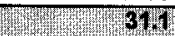 \\
\hline 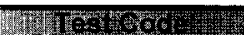 & & & & \\
\hline @TICTOC1 & & & & \\
\hline$\frac{W_{1}}{\text { LIQUID }}$ & & & & \\
\hline 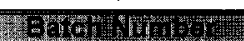 & & & & \\
\hline 96009104 & & & & \\
\hline 7. & $\mu g$ of Carbon $/ \mathrm{mL}=\left(\mathrm{C}_{1}-\mathrm{C}_{2}\right) * \mathrm{DF} / \mathrm{SS}$ & & & \\
\hline 0 & $\mu \mathrm{g}$ of Carbon $/ \mathrm{mL}$ for $\mathrm{TIC}=5$ if $\mathrm{C} 1<\mathrm{C} 2$ & & & \\
\hline 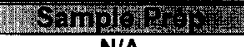 & $\mu \mathrm{g}$ of Carbon $/ \mathrm{mL}$ for TOC $=40$ if $\mathrm{C} 1<\mathrm{C2}$ & & & \\
\hline N/A & & & & \\
\hline$\frac{1}{\text { S96T002860 }}$ & & & & \\
\hline$\frac{\text { ThSHnders }}{\text { CARB2 }}$ & & & & \\
\hline 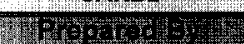 & & & & \\
\hline RGJ & & & & \\
\hline 12018 & & & & \\
\hline RWS & & & & \\
\hline 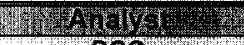 & & & & \\
\hline DEG & & & & \\
\hline 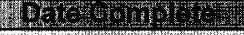 & & & & \\
\hline $09109 / 96$ & & & & \\
\hline 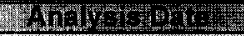 & & & & \\
\hline 09106196 & & & TIC & TOC \\
\hline 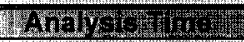 & Method Detection Limit in $\mu \mathrm{g} / \mathrm{mL}$ & & 5 & 40 \\
\hline $10.58 \mathrm{AM}$ & & & & \\
\hline 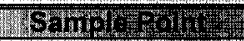 & $\mu \mathrm{g}$ of Carbon $/ \mathrm{mL}$ & & $8.98 E+03$ & $3.26 \mathrm{E}+04$ \\
\hline
\end{tabular}

\begin{tabular}{|c|c|c|c|}
\hline Data Entered By: & Bej & Date: & $09 / 09 / 96$ \\
\hline Signature of Chemist: & N Sdusedur & Date: & $9 / 9 / 96$ \\
\hline
\end{tabular}

782 
WHC-SD-WM-DP-191, REV. I

WORKBOOK PAGE: DUP4

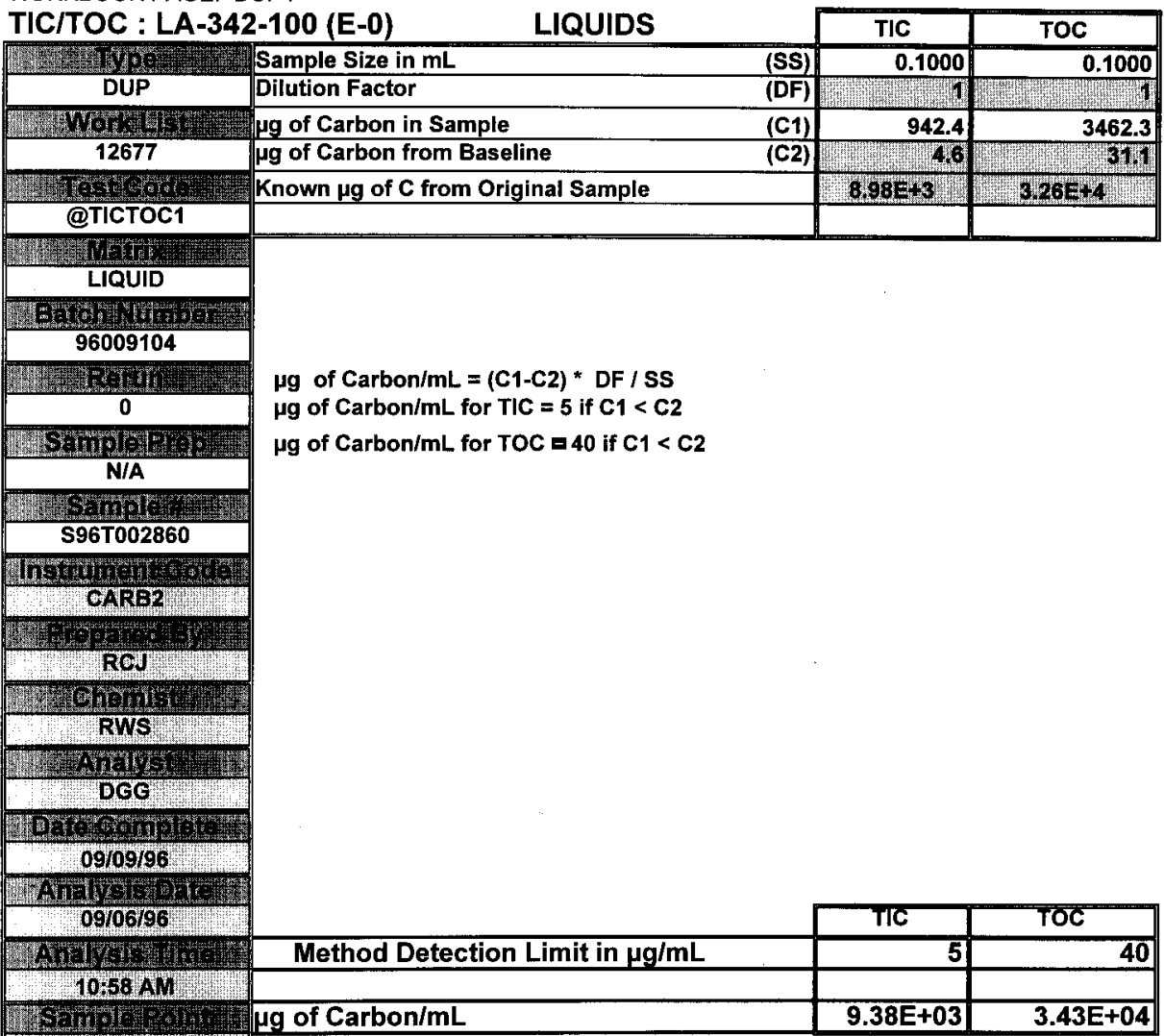

U106

\begin{tabular}{|lll||}
\hline Data Entered By: & RCJ & Date: \\
\hline Signature of Chemist: & Date: $9 / 9 / 96 / 96$ \\
\hline
\end{tabular}


WORKBOOK PAGE: SPIKE5

TIC/TOC : LA-342-100 (E-0)

\begin{tabular}{|c|c|c|c|c|}
\hline 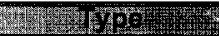 & Sample Vial Data & & TIC & TOC \\
\hline SPK & Sample Volume in $\mathrm{mL}$ & (SS) & 0.7000 & Togro00 \\
\hline WETEF & Final Coulometer Reading in $\mu \mathrm{g}$ & (C1) & 902.2 & 3295 \\
\hline 12677 & Spiked Vial Data & & & \\
\hline 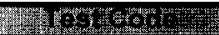 & Sample Volume in $\mathrm{mL}$ & (SPK SS) & 0.1000 & 0.1000 \\
\hline @TICTOC1 & Amount of Spike Std. in mL & (SPK VOL) & 0.500 & 0.100 \\
\hline 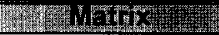 & Final Coulometer Reading in $\mu \mathrm{g}$ & (C2) & 1202.5 & 3554.2 \\
\hline LIQUID & Spike Book Number & & 22N12E & 24N12C \\
\hline 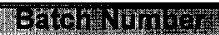 & Spike Standard Value in $\mu \mathrm{g} / \mathrm{ml}$ & (SPK CONC) & 601 & 3001 \\
\hline
\end{tabular}

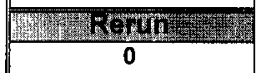

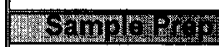

N/A

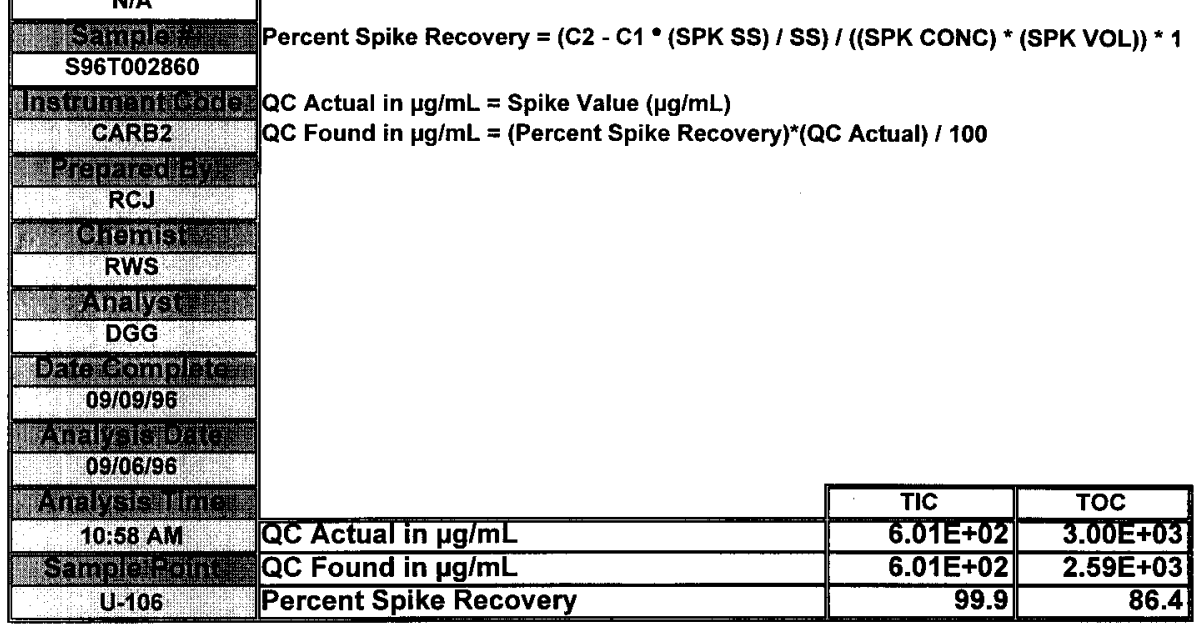

\begin{tabular}{|c|c|c|c|}
\hline Data Entered By: & RCJ & Date: & $09 / 09 / 96$ \\
\hline Signature of Chemist: & & Date: & $9 / 9 / 96$ \\
\hline
\end{tabular}

734 
WORKBOOK PAGE: SAM6

\begin{tabular}{|c|c|c|c|c|}
\hline TIC/TOC : LA-34 & $2-100(E-0)$ & & TIC & TOC \\
\hline 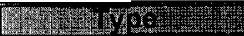 & Sample Size in $\mathrm{mL}$ & (SS) & 0.1000 & 0.1000 \\
\hline SAMPLE & Dilution Factor & (DF) & 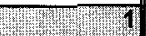 & 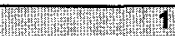 \\
\hline PW Wory & pg of Carbon in Sample & (c1) & 933.8 & 3393.4 \\
\hline 12677 & pg of Carbon from Baseline & (C2) & 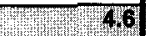 & 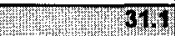 \\
\hline 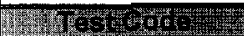 & & & & \\
\hline @TICTOC1 & & & & \\
\hline 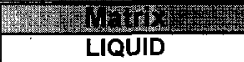 & & & & \\
\hline 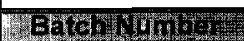 & & & & \\
\hline 96009104 & & & & \\
\hline 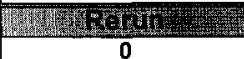 & $\begin{array}{l}\mu g \text { of Carbon } / \mathrm{mL}=\left(C 1-C_{2}\right)^{*} D F / S S \\
\mu g \text { of Carbon } / m L \text { for } T \mid C=5 \text { if } C 1<C 2\end{array}$ & & & \\
\hline 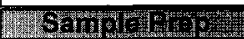 & $\mu \mathrm{g}$ of Carbon $/ \mathrm{mL}$ for TOC $=40$ if $\mathrm{C} 1<\mathrm{C} 2$ & & & \\
\hline N/A & & & & \\
\hline 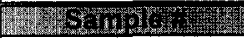 & & & & \\
\hline S96T003051 & & & & \\
\hline 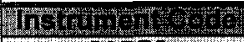 & & & & \\
\hline CARB2 & & & & \\
\hline 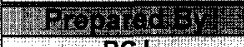 & & & & \\
\hline RCJ & & & & \\
\hline Chemist & & & & \\
\hline Finalys & & & & \\
\hline DGG & & & & \\
\hline 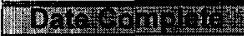 & . & & & \\
\hline $09 / 09196$ & & & & \\
\hline 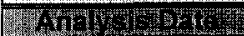 & & & & \\
\hline 0910696 & & & TIC & TOC \\
\hline 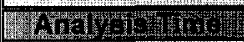 & Method Detection Limit in $\mu \mathrm{g} / \mathrm{mL}$ & & 5 & $\overline{40}$ \\
\hline $10.58 \mathrm{AM}$ & & & & \\
\hline 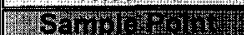 & $\mu \mathrm{g}$ of Carbon $/ \mathrm{mL}$ & & $9.29 E+03$ & $3.36 E+04$ \\
\hline
\end{tabular}

0106

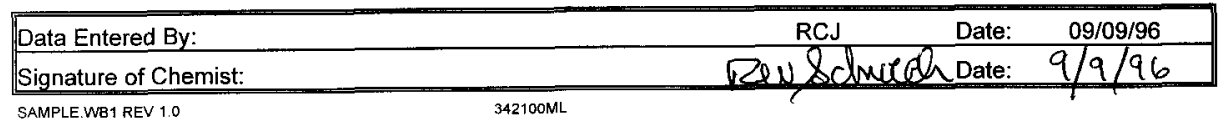


WHC-SD-WM-DP-191, REV. I

WORKBOOK PAGE: DUP7

\begin{tabular}{|c|c|c|c|c|}
\hline TIC & & & TIC & TOC \\
\hline Dry & Sample Size in $\mathrm{mL}$ & (SS) & 0.1000 & 0.1000 \\
\hline DUP & Dilution Factor & (DF) & 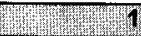 & 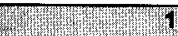 \\
\hline men & ug of Carbon in Sample & (C1) & 976 & 3377.9 \\
\hline 12677 & $\mu \mathrm{g}$ of Carbon from Baseline & (C2) & Wy & 30 \\
\hline 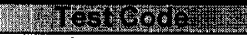 & Known $\mu \mathrm{g}$ of $\mathrm{C}$ from Origin & & $929 \mathrm{E} 3$ & 36654 \\
\hline @TICTOC1 & & & & \\
\hline
\end{tabular}

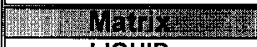

LIQUID

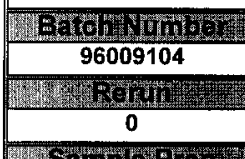

$\frac{\text { SIIII) }}{\text { N/A }}$

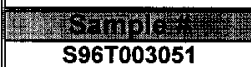

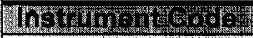

CARB2

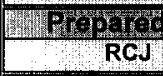

$\frac{\text { Nowili }}{\text { RW' }}$

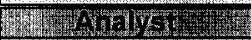

DEG

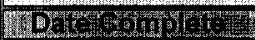

09109796

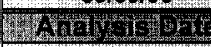

09706796

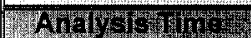

$1068 \mathrm{AH}$

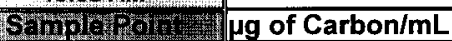

ug of Carbon $/ \mathrm{mL}=(\mathrm{C1} 1-\mathrm{C} 2)$ * DF / SS

$\mu \mathrm{g}$ of Carbon/mL for TIC $\mathrm{a} 5$ if C1 < C2

$\mu \mathrm{g}$ of Carbon $/ \mathrm{mL}$ for TOC $=40$ if $\mathrm{C}_{1}<\mathrm{C}_{2}$

\begin{tabular}{|l|r|r|}
\cline { 2 - 3 } \multicolumn{1}{c|}{} & TIC & \multicolumn{1}{c|}{ TOC } \\
\hline Method Detection Limit in $\mu \mathrm{g} / \mathrm{mL}$ & 5 & \\
\hline & & \\
\hline$\mu g$ of Carbon $/ \mathrm{mL}$ & $9.71 \mathrm{E}+03$ & $3.35 \mathrm{E}+04$ \\
\hline
\end{tabular}

\begin{tabular}{|c|c|c|c|}
\hline Data Entered By: & $\mathrm{RCJ}$ & Date: & $09 / 09 / 96$ \\
\hline Signature of Chemist: & & Date: & \\
\hline
\end{tabular}


WriC-SD-WM-DP-191, REV.

WORKBOOK PAGE: SPIKE8

TIC/TOC : LA-342-100 (E-0)

LIQUIDS

\begin{tabular}{|c|c|c|c|}
\hline 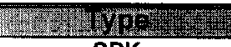 & Sample Vial Data & TIC & TOC \\
\hline SPK & Sample Volume in $\mathrm{mL}$ & MOT1000: & 0.7000 \\
\hline Wormerr & Final Coulometer Reading in $\mu \mathrm{g}$ & 933.8 & 3393.4 \\
\hline 12677 & Spiked Vial Data & & \\
\hline Hod 600 & Sample Volume in $\mathrm{mL}$ & 0.1000 & 0.1000 \\
\hline @TICTOC1 & Amount of Spike Std. in $\mathrm{mL}$ & 0.500 & 0.100 \\
\hline (7) & Final Coulometer Reading in $\mu \mathrm{g}$ & 1279.8 & 3551.7 \\
\hline LIQUID & Spike Book Number & 22N12E & 24N12C \\
\hline Batchemon & Spike Standard Value in $\mu \mathrm{g} / \mathrm{ml}$ & 601 & 3001 \\
\hline 96009104 & \multirow{16}{*}{\multicolumn{3}{|c|}{$\begin{array}{l}\text { Percent Spike Recovery }=(\text { C2 - C1 * (SPK SS) } / \text { SS }) /((\text { SPK CONC) * (SP } \\
\text { QC Actual in } \mu \mathrm{g} / \mathrm{mL}=\text { Spike Value }(\mu \mathrm{g} / \mathrm{mL}) \\
\text { QC Found in } \mu \mathrm{g} / \mathrm{mL}=(\text { Percent Spike Recovery })^{*}(\text { QC Actual }) / 100\end{array}$}} \\
\hline STHA & & & \\
\hline 0 & & & \\
\hline 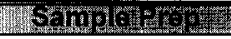 & & & \\
\hline N/A & & & \\
\hline$\frac{1}{\text { S96T003051 }}$ & & & \\
\hline$\frac{\text { MStMMMONI }}{\text { CARB2 }}$ & & & \\
\hline Wreptares: & & & \\
\hline RCJ & & & \\
\hline (obons & & & \\
\hline RWS & & & \\
\hline Harty & & & \\
\hline DGG & & & \\
\hline$\frac{109}{09 / 09796}$ & & & \\
\hline 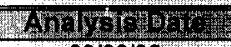 & & & \\
\hline $09 / 06 / 96$ & & & \\
\hline Analys H Ha & & TIC & TOC \\
\hline 10:58 AM & QC Actual in $\mu \mathrm{g} / \mathrm{mL}$ & $6.01 E+02$ & $3.00 \mathrm{E}+03$ \\
\hline SEMprophin & QC Found in $\mu \mathrm{g} / \mathrm{mL}$ & 6.92E+02 & $1.58 \mathrm{E}+03$ \\
\hline U,106 & Percent Spike Recovery & 115.1 & 52.7 \\
\hline
\end{tabular}

\begin{tabular}{|c|c|c|c|}
\hline Data Entered By: & RC.J & Date: & $09 / 09 / 96$ \\
\hline Signature of Chemist: & rew Qdnoran & Date: & $9 / 9 / 96$ \\
\hline
\end{tabular}


WHC-SD-WM-DP-191, REV. 1

THIS PAGE WAS INTENTIONALLY LEFT BLANK 
WHC-SD-HM-DP-191, REV. 1

THIS PAGE WAS INTENTIONALLY LEFT BLANK

789 
WHC-SD-WM-DP-191, REV. 1

THIS PAGE WAS INTENTIONALLY LEFT BLANK

790 
WHC-SD-WM-DP-191, REV. 1

$\leftarrow$

THIS PAGE WAS INTENTIONALLY LEFT BLANK 
WHC-SD-WM-DP-191, REV. I

a:-

THIS PAGE WAS INTENTIONALLY LEFT BLANK

792 
WHC-SD-WM-DP-191, REV. 1

$\leftarrow$

THIS PAGE WAS INTENTIONALLY LEFT BLANK

793 
WHC-SD-WM-DP-191, REV . 1

THIS PAGE WAS INTENTIONALLY LEFT BLANK

794 
WHC-SD-WM-DP-191, REV, I

$\approx$

THIS PAGE WAS INTENTIONALLY LEFT BLANK 
WHC-SD-WM-DP-191, REV. 1

$\leftarrow$

THIS PAGE WAS INTENTIONALLY LEFT BLANK

796 
WHC-SD-WM-DP-191, REV. 1

THIS PAGE WAS INTENTIONALLY LEFT BLANK

797 


\section{WHG-SD-WM-DP-191, REV. 1}

Data Reprocessed on 07/16/1996 10:24:43

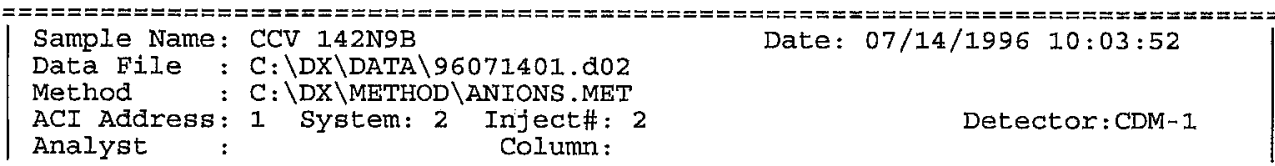

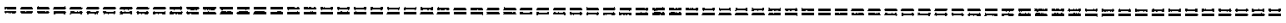

Calibration Volume Dilution Points Rate start stop Area Reject

External $\quad 1 \quad 301 \quad 3000 \quad 5 \mathrm{~Hz} \quad 0.00 \quad 10.00 \quad 200$

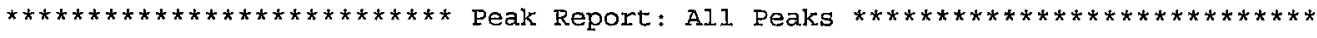

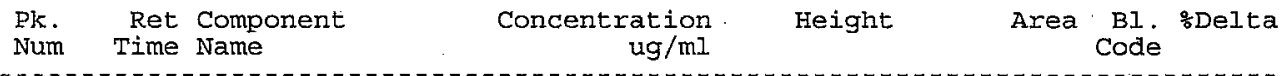

$\begin{array}{rll}2 & 0.89 & \\ 3 & 1.04 & \text { FLUORIDE } \\ 4 & 1.53 & \text { CHLORIDE } \\ 5 & 1.82 & \text { NITRITE } \\ 6 & 2.70 & \text { BROMIDE } \\ 7 & 3.08 & \text { NITRATE } \\ 8 & 4.43 & \text { PHOSPHATE } \\ 9 & 5.76 & \text { SULFATE } \\ 10 & 7.54 & \text { OXALATE }\end{array}$

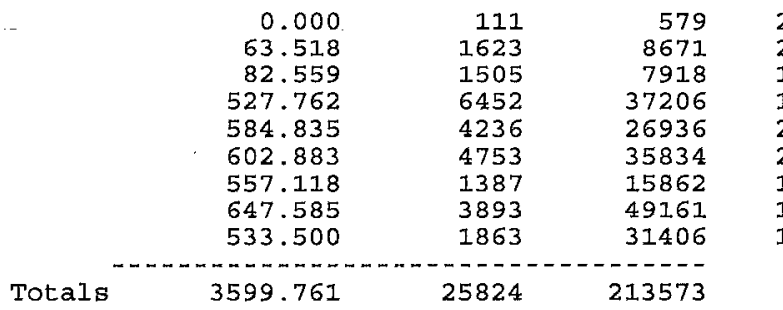

File: 96071401.d02 Sample: CCV 142N9B

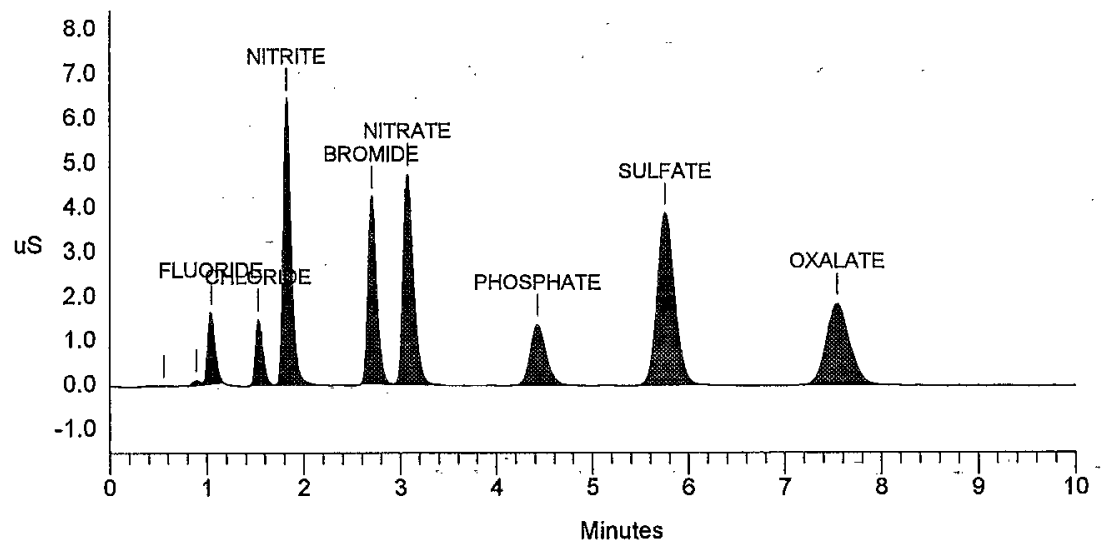




\section{LABCORE Data Entry Template for Worklist\# 10950}

Analyst:-T.WORRELC__ Instrument: ICPOT 2

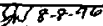

Method: IAA-505-151/161 Rev/Mod $B-1$

Worklist Comment: ICP U-106 (FUSION)

\begin{tabular}{|c|c|c|c|c|c|c|}
\hline $\mathbf{s}$ & Sample\# & $\mathbf{R A}$ & Test & Watrix & Group\# & Project \\
\hline 1 & ICV & & QICP-QC & $\mathrm{QC}$ & & \\
\hline 2 & $\mathrm{ICB}$ & & $\Theta I C P-Q C$ & $Q C$ & & \\
\hline 3 & LLLS & & $@ I C P-Q C$ & $\mathrm{OC}$ & & \\
\hline 4 & ICSA & & $@ I C P-Q C$ & $\mathrm{QC}$ & & \\
\hline 5 & ICSAB & & $@ I C P-Q C$ & QC & & \\
\hline 6 & $\begin{array}{l}\text { PREPBLKARE TTA } \\
\text { ON P-8 }-96\end{array}$ & & QICP-F01 & SOLID & & \\
\hline
\end{tabular}

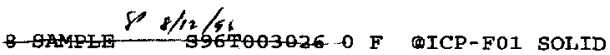
Analytes Requested : if $8 / 12 / 96$

TDIF- $59670030260 \%$
10 spk $80 \mathrm{e} / 12 / \mathrm{sg}$

$11 \mathrm{CCV}$

$12 \mathrm{CCB}$

13 SEROIL

14 SAMPLE

QICP-FO1 SOLID

@ICP-FOI SOLID

QICP-QC QC

QCP-QC QC

S96T003029 OF QICP-FO1 SOLID

S96T003029 O F OICP-FOI SOLID
Book\# $58048 \mathrm{~A}$

\begin{tabular}{|c|c|c|c|c|c|c|}
\hline$A G-E-01$ & , AL-F-OI & $A S-F-01$ & , & $B-F-01$ & BA-F-01 & , \\
\hline$B E-F-01$ & , BI-F-0I & $C A-F-01$ & . & $C D-F-01$ & $C E-F-01$ & - $\mathbf{C O}-\mathbf{F}-0 \mathbf{I}$ \\
\hline CR-F-01 & , CU-F-01 & , FE-F-01 & ' & $\mathrm{K}-\mathrm{F}-01$ & LAA-F- 01 & , LI-F-0I \\
\hline MG-F-01 & , MN-F-01 & MO-F-OI & ， & NA-F-O1 & $N D-F-O 1$ & , NI-F OI \\
\hline$P-F-01$ & , PB-F-OI & $S-F-01$ & ' & SB-F-OI & SE-F-01 & , SI-F-01 \\
\hline$S M-F-01$ & , SR-F-01 & TI-F-0I & , & $T L-F-01$ & U-F-01 & , V-F-01 \\
\hline ZN-F-01 & ZR-F-01 & & & & & \\
\hline
\end{tabular}

96000643 U-106 (P).

AS-F-01，B-F-01，BA-F-01,

$\mathrm{CA}-\mathrm{F}-01, \mathrm{CD}-\mathrm{F}-01, \mathrm{CE}-\mathrm{F}-01, \mathrm{CO}-\mathrm{F}-01$,

E-F-01, K-F-01, LA-F-01，LI-F-01,

O-F-OI，NA-F-OI，ND-F-OI，NI-F-OI.

I-F-01, TL-F-01, U-F-01, V-F-01,

$96000643 \mathrm{U}-106$ (P)

Analytos Requested: $A G-F-01, A I-F-01, A S-F-01, B-F-01$, BA-F-OI,

BE-F-01， BI-F-01，CA-F-01，CD-F-01，CE-F-01，CO-F-01 , CR-F-01， CU-F-01， FE-F-01，K-F-01 , LA-F-01， LI-F-01,

Note: Sendilfor 5965033027 needs to be multiplicd by 205 .

Data Entry Comments: Sapele sacer calcilatm:

$5967003029 \quad a \ell=23.63 \mathrm{~kg} \times 1=1.17 \mathrm{l}+4 \mathrm{~kg}$

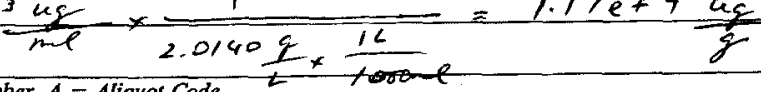

$\bar{S}=$ Worklist Slot Number, $\boldsymbol{R}=$ Replicate Number, $\boldsymbol{A}=$ Aliquot Code.

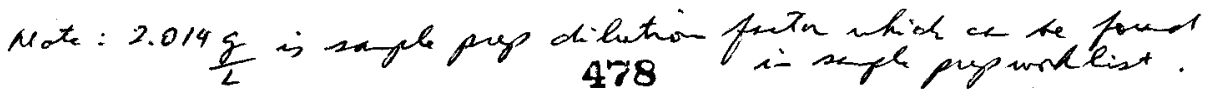


WHC-SD-WM-DP-191, REV I

WORKBOOK PAGE: SPIKE5

TIC/TOC : LA-342-100 (E-0)

SOLIDS

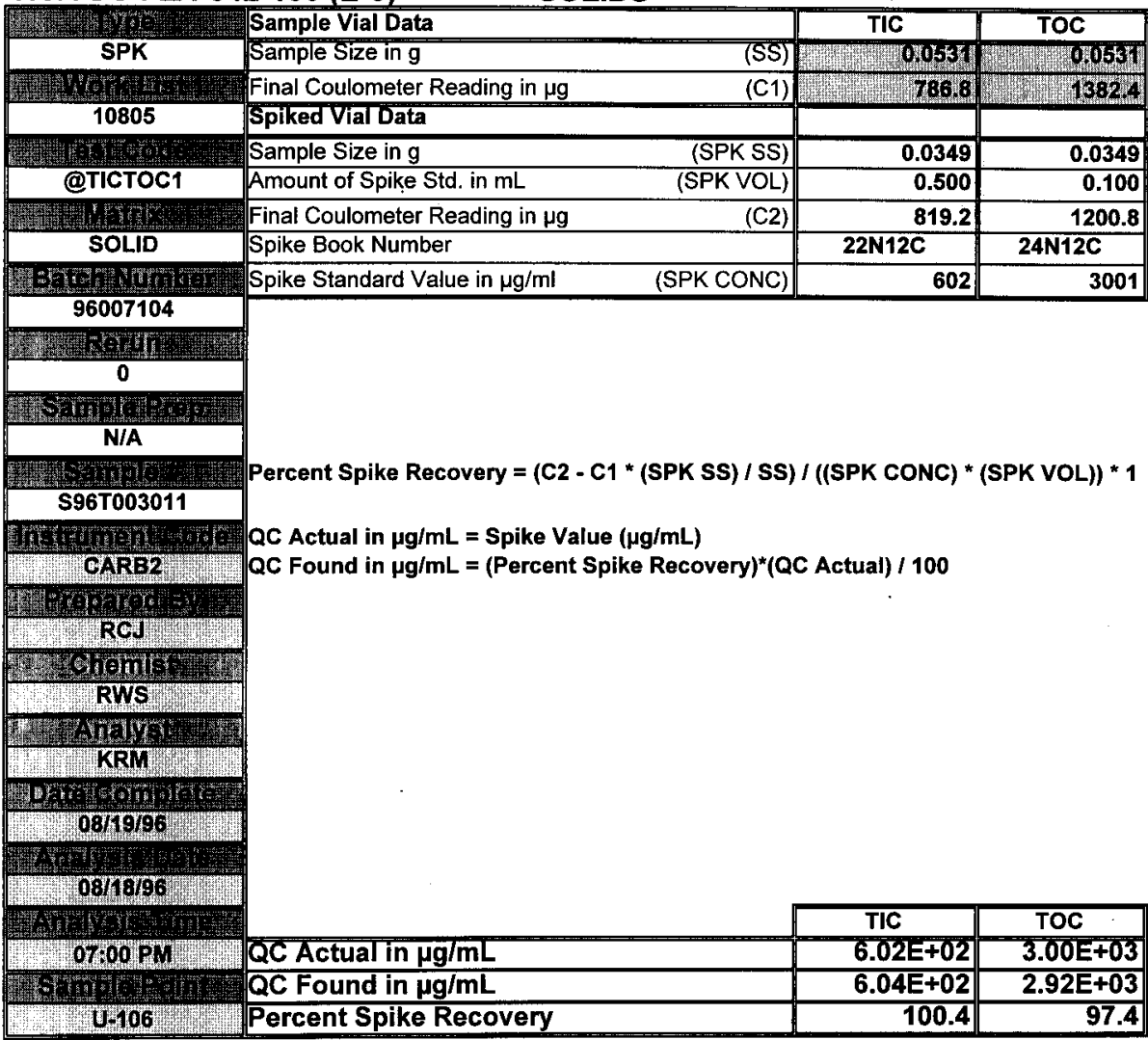

\begin{tabular}{|llll||}
\hline Data Entered By: & RCJ & Date: & $08 / 19 / 96$ \\
\hline Signature of Chemist: & & Date: & $08 / 2$ is/96 \\
\hline SPIKE.WB1 REV 1.1 & & & \\
\hline
\end{tabular}


WחL-SL-WM-UP-191, REV. I

WORKBOOK PAGE: SAM6

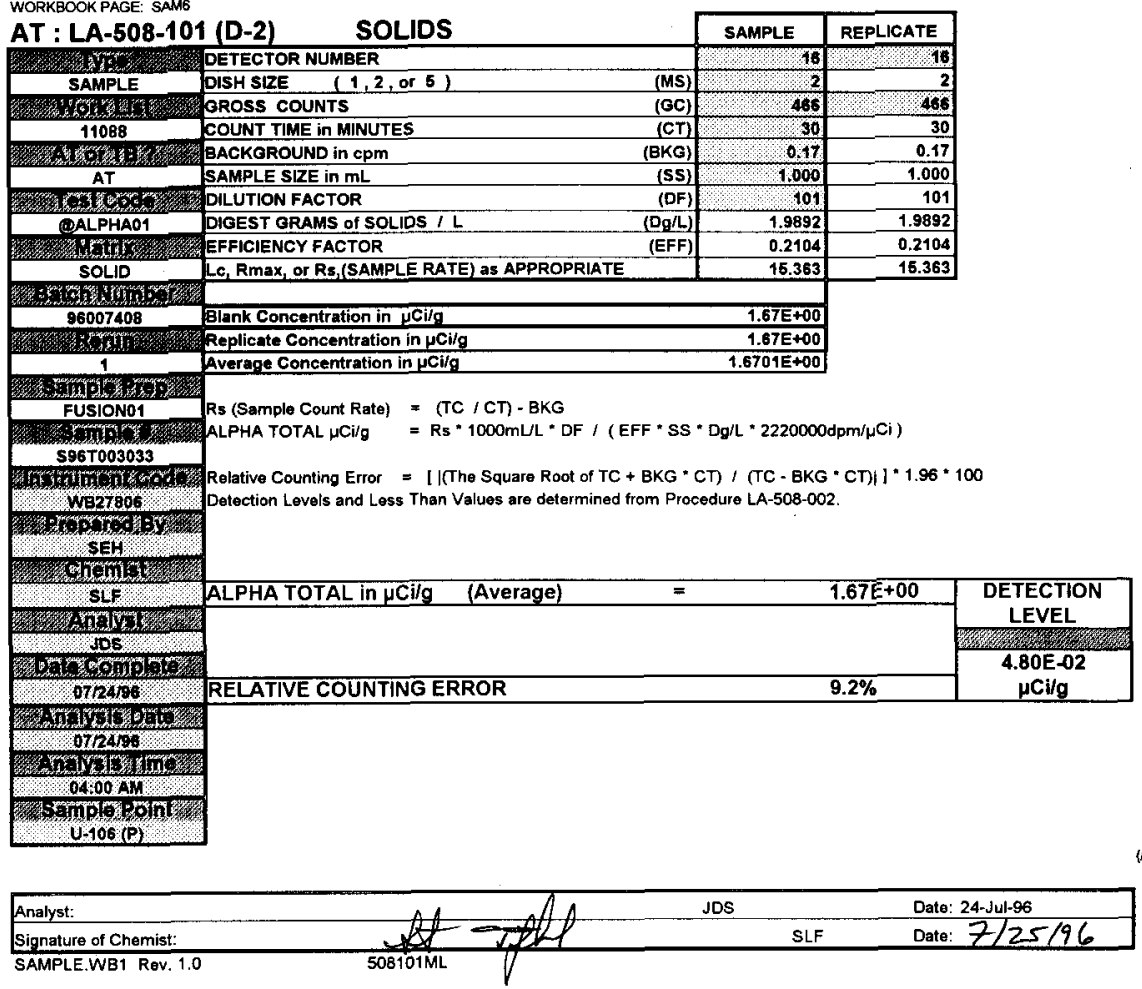


Minimun Detectable Activity Report WHC-SD-WM-DP-191, REV. I Sample ID : S96T3878-DUP

Acquisition date : 23-AUG-1996 : 3

Nuclide

$\mathrm{BE}-7$

$\mathrm{NA}-22$

NA -24

$\mathrm{K}-40$

CR- 51

MN -54

$\mathrm{CO}-56$

CO- 58

FE-59

SE-75

SR-85

$\mathrm{Y}-88$

NB-94

ZRNB-95

RU-103

RURH-106

AG $-108 \mathrm{~m}$

CD-109

$A G-110 \mathrm{M}$

SN-113

TE- $123 \mathrm{~m}$

SB-124

$S B-125$

TE- $125 \mathrm{~m}$

I-131

CS -134

$\mathrm{BA}-140$

LA- 140

CEPR-144

EU-152

HG-203

TL-208

BI -212

PB-212

BI-2 14

$\mathrm{PB}-214$

RA-224

$\mathrm{RA}-226$

$\mathrm{AC}-228$

$\mathrm{TH}-228$

TH-229

$\mathrm{PA}-233$

UTH-233

$\mathrm{PA}-234 \mathrm{M}$

TH-234

$\mathrm{U}-235$

$\mathrm{NP}-239$

PU-239

AM-241

$A M-243$
Bckgnd

Sum

2400.

134.

6.

131.

1827 .

27.

34 .

29.

19.

2100 .

1250 .

4 .

72 .

154 .

1432 .

464 .

154 .

2004 .

2428 .

2019 .

2103.

475 .

2373.

1945 .

1912 .

465 .

881 .

13.

2139 .

6.

1951 .

1987.

41 .

2278 .

482 .

1914 .

2363 .

2684 .

46 .

1912 .

1922 .

1898 .

2286 .

33 .

1629.

2666 .

2092 .

2077.

1771 .

1785 .
Energy

(kev)

477.59

1274.53

1368.55

1460.75

320.08

834.83

846.76

810.78

1099.25

264.66

514.01

1836.06

871.09

724.18

497.08

621.93

722.94

88.03

657.76

391.69

159.00

602.73

427.89

109.27

364.48

604.70

537.31

1596.21

133.51

1408.01

279.20

277.36

727.18

238.63

609.31

351.92

240.99

186.10

911.21

84.37

88.47

312.17

245.34

1001.03

63.29

185.71

106.12

129.30

59.54

74.67
MDA

( $\mathrm{UCi} / \mathrm{L}$ )

$6.4554 \mathrm{E}+00$

$3.6318 \mathrm{E}-01$

$8.1703 E-02$

$3.7449 \mathrm{E}+00$

$4.2388 \mathrm{E}+00$

1. $1372 \mathrm{E}-01$

1. $2872 \mathrm{E}-01$

$1.1483 \mathrm{E}-01$

$2.1430 \mathrm{E}-01$

$6.8089 \mathrm{E}-01$

5. $3275 \mathrm{E}-01$

8. 3936E-02

1. $9200 \mathrm{E}-01$

$5.5011 \mathrm{E}-01$

$5.8553 \mathrm{E}-01$

$7.3832 \mathrm{E}+00$

$2.6424 \mathrm{E}-01$

$1.0261 \mathrm{E}+01$

9.2849E-01

8. $1704 \mathrm{E}-01$

3. $2660 \mathrm{E}-01$

$3.6888 \mathrm{E}-01$

$2.0683 E+00$

$9.9389 \mathrm{E}+01$

$5.9448 \mathrm{E}-01$

3. $6672 \mathrm{E}-01$

$1.8281 \mathrm{E}+00$

$1.4232 E-01$

$4.8422 \mathrm{E}+00$

$3.9850 \mathrm{E}-01$

$4.8939 \mathrm{E}-01$

$6.3447 \mathrm{E}+00$

$1.8734 \mathrm{E}+00$

$8.8192 \mathrm{E}-01$

$8.1844 \mathrm{E}-01$

$2.3431 E+00$

$9.9731 \mathrm{E}+00$

$9.7035 \mathrm{E}+00$

$6.0089 \mathrm{E}-01$

3. $0870 \mathrm{E}+01$

$1.4565 \mathrm{E}+00$

$1.1068 \mathrm{E}+00$

$3.6766 \mathrm{E}+02$

2. $5065 E-01$

$1.9188 \mathrm{E}+01$

$5.8776 \mathrm{E}-01$

1. $3081 \mathrm{E}+00$

4. $1529 E+03$

3. $5315 \mathrm{E}+00$

8. $3327 \mathrm{E}-01$ 
WHC-SD-WM-DP-191, REV. 0

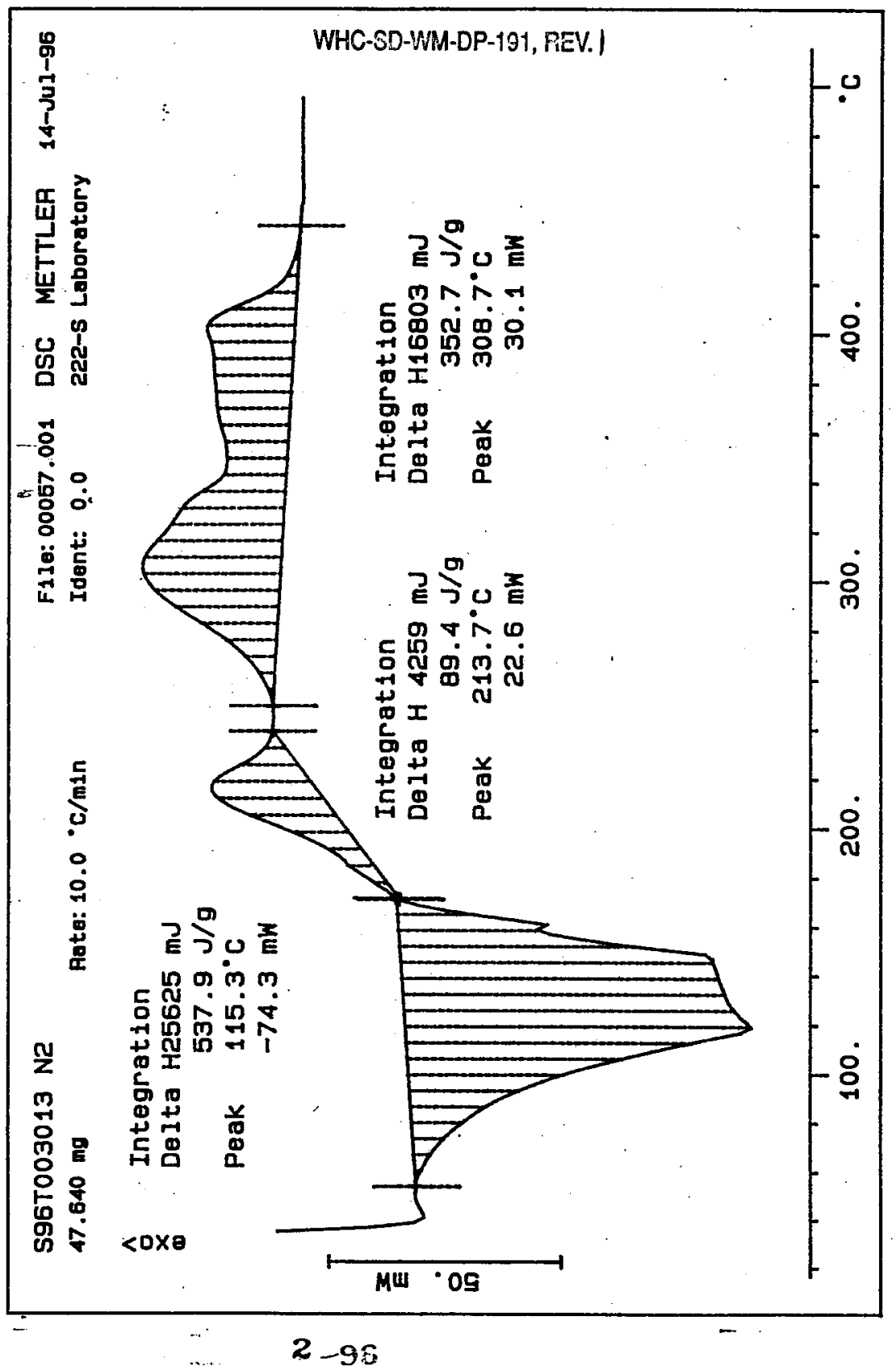

